\title{
Investigating knowledge and use of technical vocabulary in Traditional Chinese Medicine
}

\author{
By
}

Cailing Lu

\begin{abstract}
A thesis
submitted to the Victoria University of Wellington

in fulfilment of the requirements for the degree of

Doctor of Philosophy

in Applied Linguistics
\end{abstract}

Victoria University of Wellington

2018 


\begin{abstract}
This research investigates the nature of vocabulary, especially technical vocabulary, in the specialized discipline of Traditional Chinese medicine (TCM), which is an important area of higher education. It consists of three linked studies in correspondence to three research aims using a combination of quantitative and qualitative methods. Study 1 addressed the questions of what kinds of words constitute TCM lexis given its origin, and what is the vocabulary load of Englishmedium texts in this discipline. To answer these questions, a series of lexical analyses was conducted on three corpora: theory-based and practice-based textbook corpora and a journal article corpus, which reflect the main areas of reading for TCM students. The results showed that while high, mid and lowfrequency vocabulary make up a fairly large proportion of these texts, other lexical items such as abbreviations, loan words, medical words, proper nouns, and compounds also feature in them, but in differing proportions depending on the text types. Further, this study found that a large vocabulary of 13,000 word families plus four supplementary lists and two TCM-specific lists is needed. This is the point which most TCM learners can read TCM textbooks and journal articles without vocabulary being a handicap.
\end{abstract}

Study 2 looked more closely at the technical vocabulary in TCM. The nature of technical vocabulary was explored and TCM technical word lists of both single and multiword units were developed for learners and teachers in this discipline. A total of 2,778 word types were selected for the TCM technical word list based on the criteria of relative keyness in the TCM Corpora compared to a general written English corpus, meaningfulness, and frequency. The list provided $36.65 \%$ coverage of the corpora from which it was developed. In addition, a TCM technical lexical bundle list with 898 bundles was developed to supplement the technical word list. The findings suggested that lexical bundles play an essential role in creating meaning and structure of TCM discourse. Thus, they should be regarded as a basic linguistic construct since some technical vocabulary needs to be seen in bundles rather than in single words. 
The last study bridged the gap between corpus-based word lists and the actual ESP vocabulary learning context by way of investigating learners' understanding of the technical words from the technical word list generated from the second study. Results suggested that learners faced different challenges in technical vocabulary learning depending on their linguistic backgrounds. Specifically, Chinese learners had great difficulty with technical words from the lowerfrequency bands of BNC/COCA word lists, while Western learners encountered challenges with loan words borrowed from Chinese. As a result, a certain divergence between the Western and Chinese TCM learners' understanding of technical words was manifested. These findings indicate that a pedagogically useful word list should be adaptable to learners from different linguistic backgrounds.

Drawing on these findings, this thesis also provides methodological, theoretical, and pedagogical implications so that the TCM learners can gain better support in their specialized English vocabulary learning. They can also enable the teachers and course designers to better scaffold their students' vocabulary development. 


\section{Acknowledgements}

I owe several debts of gratitude to my supervisors, colleagues, friends, and family for their help, support and encouragement in the completion of this thesis.

First, I would like to express my deepest gratitude to my primary supervisor Associate Professor Averil Coxhead, and secondary supervisors - Associate Professor Frank Boers and Associate Professor Peter Gu, for their invaluable guidance, generous support and continual encouragement during my whole $\mathrm{PhD}$. It is my greatest honor and privilege to work with them. They have helped me grow as a researcher.

I would also like to express my sincerest gratitude to Dr. Philip Durrant, who supervised for my Master's project. He has greatly influenced my way of thinking and direction of academic life. Without him, I would never have considered doing a $\mathrm{PhD}$ in vocabulary.

My special thanks goes to all participants for generously contributing their time to this research project. My warmest thanks also goes to Xuewei Li, Barbara Hoverd, Chito Pantig and Debra Betts, who have helped me with contacting the participants. My great thanks go to Dr. Betsy Quero, for her generosity in sharing her medical textbook corpus with me. I am also grateful to Tony Quinn, who is my subject librarian, for his help with purchasing the COCA corpus and other materials that I needed. Without their help, this thesis could hardly have been written.

It is my privilege to study in School of Linguistics and Applied Language Studies at Victoria University of Wellington (VUW). It is a friendly, supportive, and professional place for research. Further, I am especially indebted to VUW for financial support during my whole $\mathrm{PhD}$ in the form of a Victoria Doctoral Scholarship and Faculty Research Grants. 
My heartfelt thanks also go to my cousins - Yu Lu and Yuchen Wang for their help with conversion of textbooks from PDF to TEXT files. This saved me a considerable amount of time and prevented my eyes from further damage.

In addition, I am very grateful to all friends in New Zealand and China who have helped me during the way - the colleagues at VUW, such as Associate Professor Meredith Marra, Janet Attrill, Brian Strong, Yen Dang, Lauren Whitty, Judy Wei; and also my dearest friends in China. They have made my $\mathrm{PhD}$ life a more pleasant journey.

Last but not least, I am extremely grateful to my dearest parents, grandparents, and lovely younger sister for their love, support, and trust. I dedicate this work to them. 


\section{Table of contents}

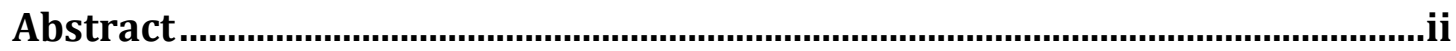

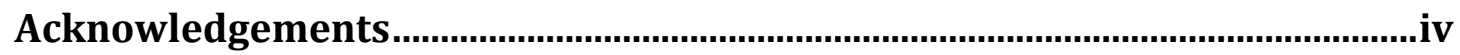

Table of contents........................................................................................

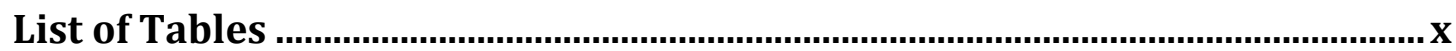

List of Figures...................................................................................... xii

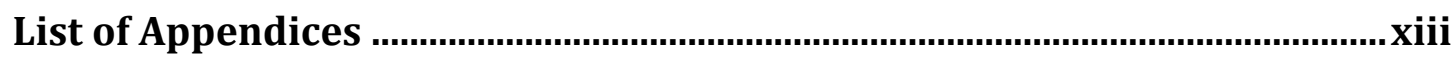

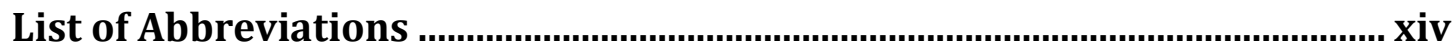

Chapter 1 Introduction ....................................................................................1

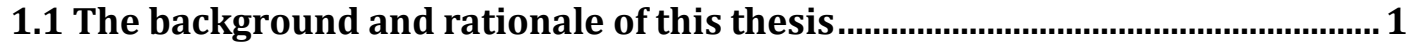

1.2 The aims of this thesis .............................................................................................. 4

1.3 The organization of this thesis .......................................................................... 6

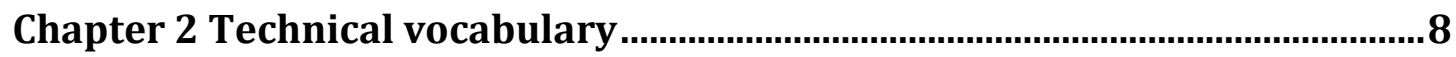

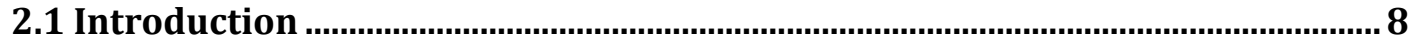

2.2 What is technical vocabulary? ……………………............................................. 8

2.3 How to identify technical vocabulary in specialized texts? ................................10

2.3.1 Corpus-based approach.................................................................................................. 10

2.3.2 Semantics-based approach ……………………………………………………………….... 11

2.3.3 Mixed methods ............................................................................................................. 13

2.4 What is the coverage of technical vocabulary in specialized texts?.................14

2.5 What is involved in knowing a technical word? ..................................................15

2.5.1 Knowing general aspects of vocabulary ............................................................ 15

2.5.2 Understanding the subject knowledge........................................................................ 16

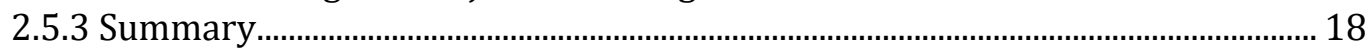

2.6 What might make a technical word difficult to learn? ........................................18

2.7 Technical vocabulary beyond single word units: Lexical bundles ....................21

2.7.1 What are 'lexical bundles'? ......................................................................................2 22

2.7.2 How to identify lexical bundles in the specialized texts? ...................................... 22

2.7.3 Lexical bundle studies in disciplinary contexts........................................................ 23

2.8 Summary...........................................................................................................25

Chapter 3 Word lists and vocabulary learning ……....................................... 28

3.1 Introduction ..................................................................................................28

3.2 Where is technical vocabulary situated in vocabulary learning? ......................28

3.3 Developing and using word lists .........................................................................30

3.3.1 The essential units of counting in word list studies ................................................. 31

3.3.2 General high-frequency word lists ............................................................................. 34

3.3.3 Generic academic word lists........................................................................................ 37

3.3.4 Discipline-specific word lists ......................................................................................... 39

3.3.5 Medicine-specific word lists...................................................................................... 41

3.4 Making use of the existing word lists: Vocabulary profile analysis ..................46

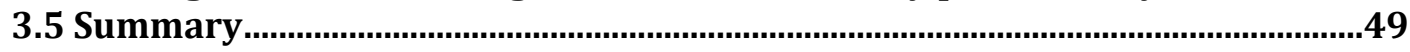

Chapter 4 Lexical profile and vocabulary loads of TCM texts ..........................52

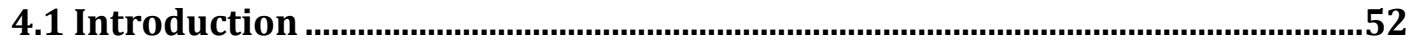

4.2 Building the TCM Corpora ………………………………………………………...53

4.2.1 How to build a representative corpus? ..................................................................... 53

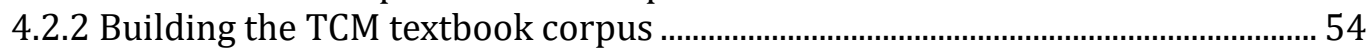


4.4 Data analysis

4.4.1 The instrument for analysis.................................................................................... 59

4.4.2 Processing the TCM Corpora........................................................................................ 60

4.4.3 Adapting the existing BNC/COCA base word lists.................................................... 62

4.4.4 Categorizing the remaining 'Not in the lists' word types ...................................... 63

4.4.5 Procedure ................................................................................................................ 64

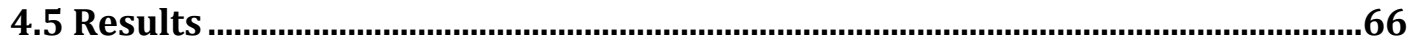

4.5.1 Lexical profile of the TCM texts along Nation's (2012) BNC/COCA lists .......... 66

4.5.2 TCM-specific vocabulary in TCM Corpora .............................................................. 70

4.5.3 Coverage of high, mid, and low-frequency vocabulary in TCM............................. 72

4.5.4 Vocabulary load of the TCM texts............................................................................... 74

4.5.5 Comparison of lexical profile in TCM and Western Medicine ............................... 76

4.5.6 Comparison of lexical profile in TCM and general written English..................... 78

4.6 Summary of the findings and rationale for next study ....................................80

Chapter 5 Technical vocabulary in Traditional Chinese Medicine ................ 83

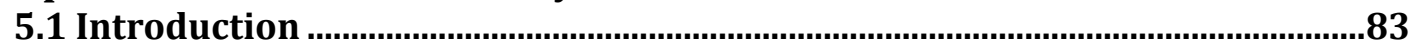

5.2 Identifying technical words in 1-25 BNC/COCA base word lists ......................84

5.2.1 Deciding unit of counting................................................................................... 85

5.2.2 Developing the comparison corpus for keyword analysis.................................... 85

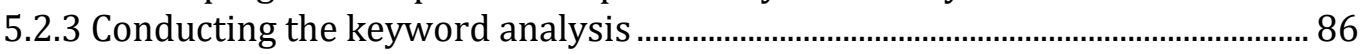

5.2.4 Developing the semantic rating scale .................................................................... 88

5.2.5 Applying the semantic rating scale and analyzing the semantic characteristics

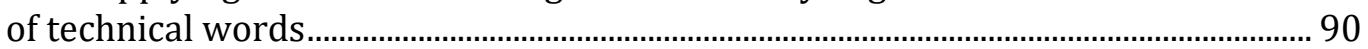

5.3 Developing the TCM technical word list............................................................90

5.3.1 Establishing the characteristics for the TCM technical word list........................ 92

5.3.2 Setting criteria for the items in the TCM technical word list.............................. 94

5.3.3 Selecting items for and validating the TCM technical word list........................... 95

5.3.4 Specifications for the TCM technical word list ....................................................... 96

5.4 Developing the TCM technical lexical bundle list ....................................................98

5.4.1 Deciding the length of lexical bundles and word unit of analysis........................ 98

5.4.2 Extracting the lexical bundles ...................................................................................... 98

5.4.3 Deciding frequency criterion for TCM technical bundle list ................................ 99

5.4.4 Deciding meaning criterion for TCM technical bundle list .................................100

5.4.5 Refining the TCM technical lexical bundle list ......................................................101

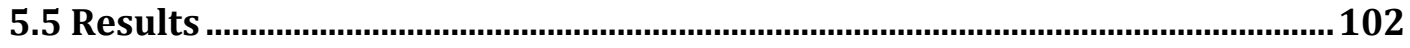

5.5.1 TCM technical words from 1 to 25 BNC/COCA word lists .................................102

5.5.2 The semantic features of BASEWRD 1-25 technical words in the TCM texts105

5.5.3 How large is the technical vocabulary of TCM? ....................................................107

5.5.4 The most frequent technical words in TCM ........................................................107

5.5.5 Coverage of the TCM technical word list in TCM theory and practice-based

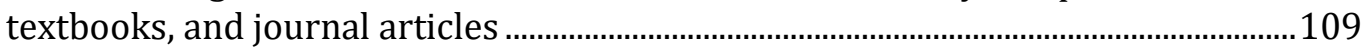

5.5.6 Coverage of the TCM technical word list in an independent collection of TCM

texts, Western Medical texts, and general written English texts..................................110

5.5.7 The most frequent technical lexical bundles in TCM .........................................111

5.6 Summary of main findings and rationale for next study.................................115

\section{Chapter 6 Investigating learners' understanding of technical vocabulary}

6.1 Introduction

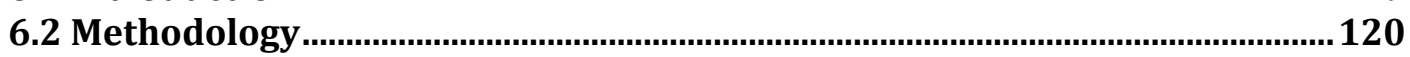

6.2.1 Participants ................................................................................................. 120

6.2.2 Word association task ................................................................................................123

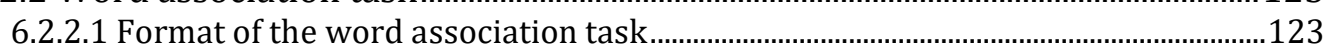

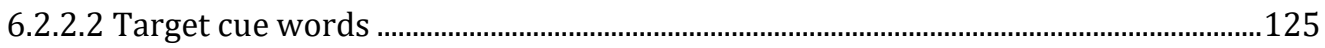


6.2.2.3 Associations and distractors.

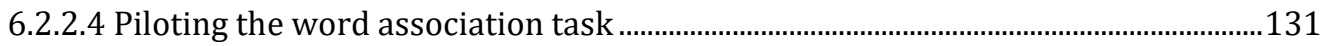

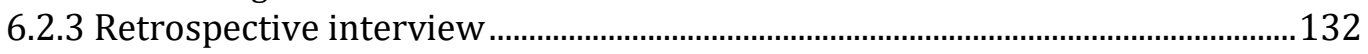

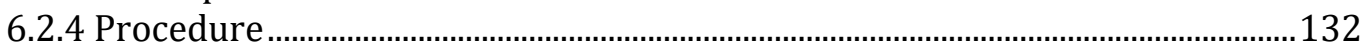

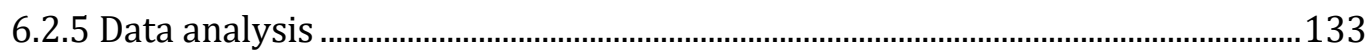

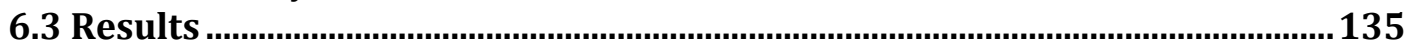

6.3.1 Outcome of the word association task ....................................................................135

6.3.1.1 Performance of Chinese TCM learners in the word association task .....................136

6.3.1.2 Performance of Western TCM learners in the word association task ....................138

6.3.2 Comparison of typical associations between Chinese and Western learners

6.3.3 Factors contributing to the divergence of typical associations ......................... 141

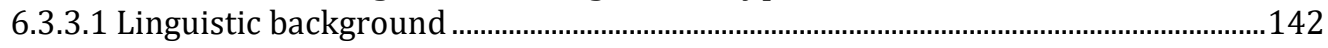

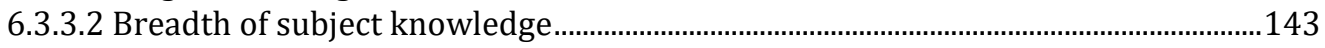

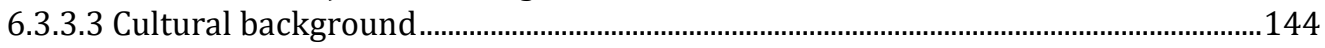

6.3.4 Divergence in understanding of technical words between Chinese and

Western learners with same typical associations.

6.4 Summary .................................................................................................................. 148

Chapter 7 Discussion ........................................................................................151

7.1 Introduction ............................................................................................................... 151

7.2 What is the value of technical vocabulary in TCM texts? .............................151

7.2.1 How much coverage does technical vocabulary provide? ..................................151

7.2.2 How important is TCM technical vocabulary in subject knowledge

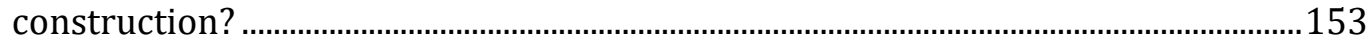

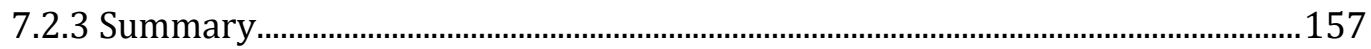

7.3 How important is learners' understanding of technical words in ESP? ...... 157

7.3.1 What makes a TCM technical word difficult for the learners? ...........................158

7.3.1.1 Chinese background TCM learners .................................................................................158

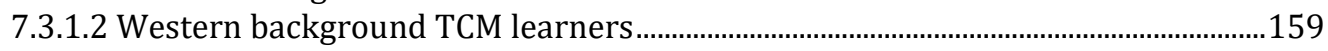

7.3.1.3 Summary................................................................................................................................. 160

7.3.2 How did TCM learners acquire knowledge of technical vocabulary? ..............160

7.3.3 How important is learners' background in their ESP vocabulary development?

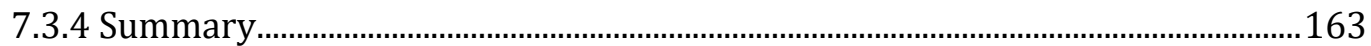

7.4 How can discipline-specific word lists better support ESP vocabulary development?

7.4.1 How can TCM technical word list better serve TCM learners' needs?.............163

7.4.2 Should technical words from general high-frequency vocabulary be included in the discipline-specific word lists? ................................................................................165

7.4.3 Should fully technical words be included in the discipline-specific word lists?

7.4.4 How can word lists be adaptable to learners' linguistic backgrounds and proficiency levels? ...........................................................................................................167

7.5 How does TCM vocabulary relate to general English vocabulary? ................ 169

7.5.1 What is the importance of the high, mid, and low-frequency vocabulary in

TCM vocabulary learning?...............................................................................................169

7.5.2 What is the value of vocabulary load analysis in understanding TCM texts?171

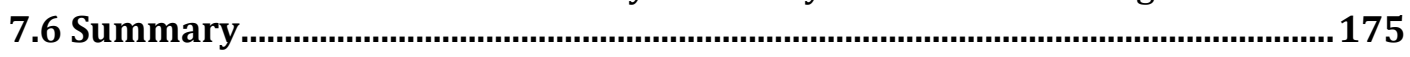

Chapter 8 Conclusion ...................................................................................177

8.1 Introduction ............................................................................................................... 177

8.2 Methodological and theoretical contributions ................................................ 178

8.2.1 Methodological contributions..................................................................................178

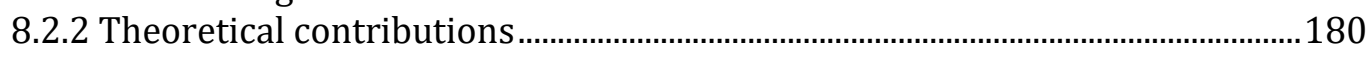




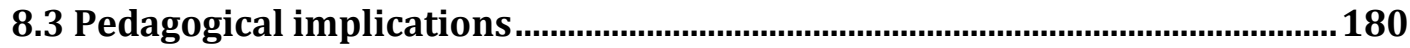

8.3.1 Developing TCM learners' knowledge of technical words..................................181

8.3.2 Developing learners' knowledge of technical lexical bundles...........................184

8.3.3 Integrating learners' sources of difficulty in word list implementation ..........185

8.3.4 Enhancing learners' knowledge of general high-frequency vocabulary.........186

8.3.5 Summary .......................................................................................................... 187

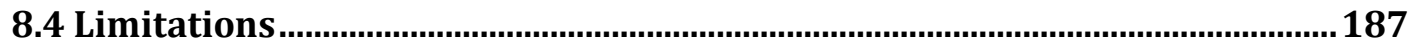

8.4.1 Limitations related corpora …………………..................................................187

8.4.2 Limitations caused by the Chinese loan words ...................................................188

8.4.3 Limitations related Nation's (2012) BNC/COCA word lists ................................189

8.4.4 Limitations caused by practical issues................................................................190

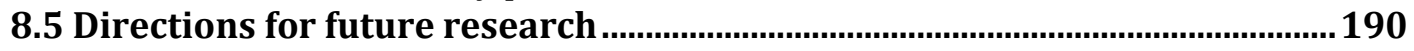

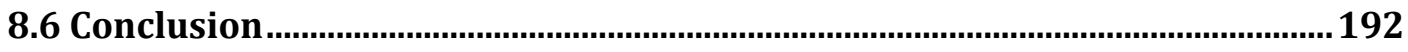

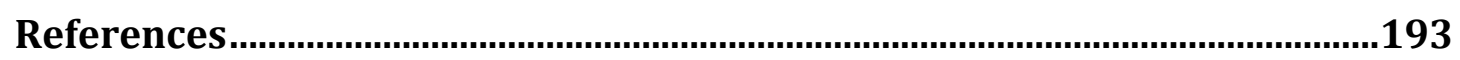

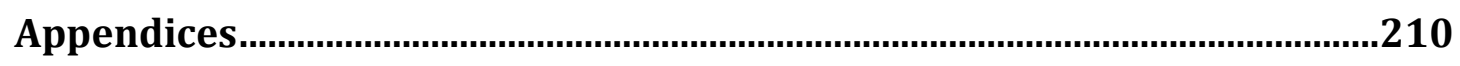




\section{List of Tables}

Table 2.1: Components of technical words in pharmacology (Fraser, 2013) 9

Table 3.1: Bauer and Nation's (1993, p. 257-p. 262) word family levels...... 33

Table 3.2: Coverage of West's (1953) GSL in different specialized corpora 36

Table 3.3: Coverage of Coxhead's (2000) AWL in different academic

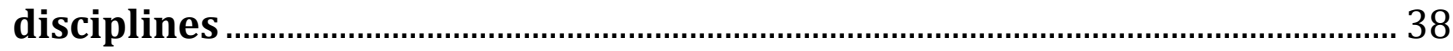

Table 3.4: Discipline-specific word lists on top of general vocabulary ......... 40

Table 3.5: Stand-alone discipline-specific word lists.......................................... 40

Table 3.6: Overview of four word lists in medicine ............................................ 41

Table 3.7: Summary of vocabulary load studies ……........................................... 48

Table 4.1: Overview of TCM theory-based textbook sub-corpus..................... 55

Table 4.2: Overview of TCM practice-based textbook sub-corpus................... 55

Table 4.3: Overview of TCM journal article corpus by journals ....................... 57

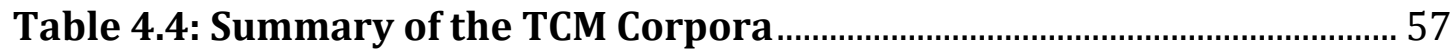

Table 4.5: Overview of Western Medical Corpora ……........................................ 58

Table 4.6: Nation's (2012) BNC/COCA base word lists...................................... 59

Table 4.7: Lexical profile of the TCM Corpora across BNC/COCA word lists67

Table 4.8: Lexical profile of TCM base word lists ................................................... 70

Table 4.9: Examples of loan words from Chinese in the TCM corpora........... 71

Table 4.10: Coverage of high, mid and low-frequency vocabulary in the TCM

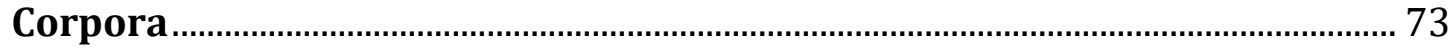

Table 4.11: Base word lists in Schmitt and Schmitt's (2014) frequency

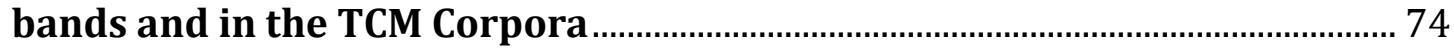

Table 4.12: Cumulative coverage for the TCM texts ........................................... 75

Table 4.13: Comparison of high-, mid-, and low-frequency vocabulary in

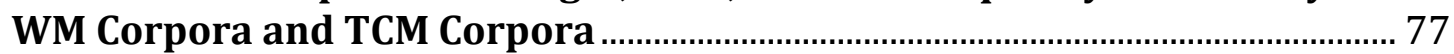

Table 4.14: Comparison of high-, mid-, and low-frequency vocabulary in

TCM Corpora and in Wellington Written Corpus ................................................... 79

Table 5.1: Overview of the comparison corpus....................................................... 86

Table 5.2: Top 20 keywords of TCM Corpora from the pilot keyword

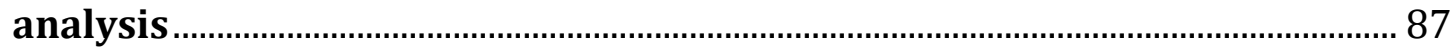

Table 5.3: Overview of the semantic rating scale................................................ 89

Table 5.4: Sub-lists of the TCM technical word list............................................. 92

Table 5.5: Three levels of each sub-list in the TCM technical word list ........ 93

Table 5.6: Comparison of coverage and number of items in five pilot lists

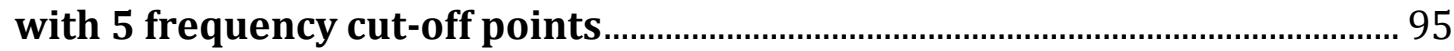

Table 5.7: Specifications for the TCM technical word list.................................. 97

Table 5.8: The most frequent 20 lexical bundles in the TCM Corpora......... 99

Table 5.9: Distribution of BASEWRD 1-25 technical words across high, mid,

and low-frequency vocabulary bands ............................................................102

Table 5.10: Top 50 TCM technical words from Nation's (2006) BNC/COCA

word lists

Table 5.11: Semantic features of technical words from BASEWRD 1-25 of

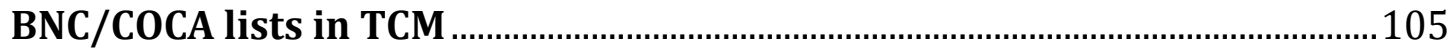

Table 5.12: Summary of technical words in TCM ...........................................107

Table 5.13: The most frequent 20 items in the TCM technical word list....108

Table 5.14: Coverage of the TCM technical word list ........................................108 
Table 5.15: Number of word types and coverage of technical words at each level of sub-lists

Table 5.16: Coverage of the TCM technical word list in different types of

TCM texts

Table 5.17: Coverage of the TCM technical word list in three validating corpora

Table 5.18: The most frequent 20 condensed lexical bundles in the TCM

Corpora.

Table 5.19: Composition of the TCM technical lexical bundle list.

Table 5.20: Summary of structures of lexical bundles at Category 3 ...........115

Table 6.1: Chinese TCM learners' demographic information

Table 6.2: New Zealand TCM learners' demographic information

Table 6.3: Categories and subcategories of word association responses

(Adapted from Fitzpatrick, 2006, p. 131)

Table 6.4: Scoring criteria for the word association task .................................134

Table 6.5: Descriptive statistics of Chinese learners' performance in the

word association task $(\mathrm{N}=11)$.

Table 6.6: Descriptive statistics of English trained and non-English trained

subgroups.

Table 6.7: Descriptive statistics of the word association task $(\mathrm{N}=10)$ from

the Western learners

Table 6.8: Summary of divergence in each category of TCM technical words 


\section{List of Figures}

Figure 5.1: Framework for analyzing semantic features of TCM technical words

Figure 6.1: Instructions for the word association task .124 Figure 6.2: Martinez's (2013, p, 190) Frequency-Transparency Framework 126

Figure 6.3: The Frequency-Transparency Framework for technical words

Figure 7.1: Division of the TCM technical word list . 168

Figure 8.1: Learning sequence of the TCM technical word list 182 


\section{List of Appendices}

Appendix 1. Results of vocabulary burden analysis.........................................210

Appendix 2. Sub-list 1 of the TCM technical word list at Level 1 ..................211

Appendix 3. Sub-list 1 of the TCM technical word list at Level 2 ...................216

Appendix 4. Sub-list 1 of the TCM technical word list at Level 3 ...................219

Appendix 5. Sub-list 2 of the TCM technical word list at Level 1 ..................220

Appendix 6. Sub-list 2 of the TCM technical word list at Level 2 ...................224

Appendix 7. Sub-list 2 of the TCM technical word list at Level 3 ..................228

Appendix 8. Sub-list 3 of the TCM technical word list at Level 1 ..................229

Appendix 9. Sub-list 3 of the TCM technical word list at Level 2 ……….......231

Appendix 10. Sub-list 3 of the TCM technical word list at Level 3.................236

Appendix 11. TCM Technical lexical bundles at Category 1 ………………......237

Appendix 12. TCM Technical lexical bundles at Category 2 ………………....239

Appendix 13. TCM Technical lexical bundles at Category 3 ............................242

Appendix 14. Word association task …………………………………………...26

Appendix 15. Ethics approval ................................................................................226

Appendix 16. Information sheet ……………………………………………...269

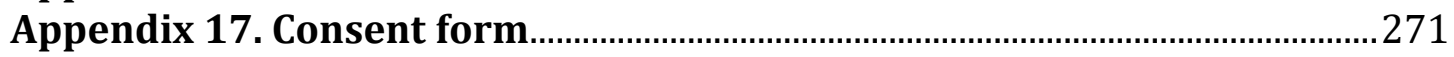

Appendix 18. Distribution of the most frequent 100 technical words in the

Frequency-Transparency Framework ………………………………………...273

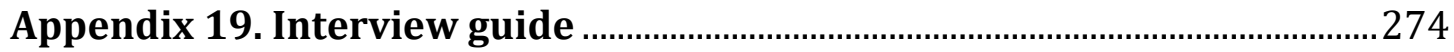

Appendix 20. Sample of interview data from a Chinese TCM learner

(English Translation) …………….........................................................................2 


\section{List of Abbreviations}

\begin{tabular}{|l|l|}
\hline Abbreviation & Full form \\
\hline AVL & Gardner and Davies's (2014) Academic Vocabulary List \\
\hline AWL & Coxhead's (2000) Academic Word List \\
\hline BASEWRD & Nation's (2012) BNC/COCA base word list \\
\hline BNC & British National Corpus \\
\hline COCA & Corpus of Contemporary American-English \\
\hline EAP & English for Academic Purposes \\
\hline EFL & English as a foreign language \\
\hline ESP & English for Specific Purposes \\
\hline GSL & West's (1953) General Service List \\
\hline L1 & First language \\
\hline L2 & Second language \\
\hline M & Mean \\
\hline MAVL & Medical Academic Vocabulary List \\
\hline MAWL & Medical Academic Word List \\
\hline MWL & Medical Word List \\
\hline RQ & Research question \\
\hline SD & Standard deviation \\
\hline TCM & Traditional Chinese Medicine \\
\hline TOPRA model & 'type of processing-resource allocation' model \\
\hline UWL & \\
\hline WM & Western Medicine \\
\hline & \\
\hline
\end{tabular}




\section{Chapter 1 Introduction}

\subsection{The background and rationale of this thesis}

Traditional Chinese Medicine (TCM) is an important area of higher education owing to its development internationally. In China, there are more than 20 TCM universities, and over 100 other universities provide TCM degree courses. These degree programs have attracted a huge number of Chinese-speaking students, and non-Chinese speaking students such as Thai, American, Japanese, Malaysian and African. The medium of instruction for Chinese-speaking students is mainly Chinese. Despite this, the Chinesespeaking students are required to learn specialized English as they progress in their study so that they can be prepared to undertake academic communication in English in global settings. For non-Chinese speaking students, English is the medium of instruction. Meanwhile, it has becoming increasingly more common for institutions in Englishspeaking countries, such as New Zealand and Australia, to include TCM as a degree course due to its growing use in Western contexts. For instance, University of Technology, Sydney provides the degree Bachelor of Health Science in Traditional Chinese Medicine. English-medium teaching and learning in this discipline thus becomes more necessary and common.

In order to understand the specialized English-language needs of TCM learners, I conducted a small-scale needs analysis with 63 Chinese-speaking learners and two lecturers from a Chinese TCM university as the thesis for my Master's (Lu, 2014). This study revealed limited technical vocabulary knowledge to be a major obstacle in specialized language learning from both learners and lecturers' perspectives. Further, the lecturer participants stated that they were unsure which vocabulary items they needed to help their learners with for them to comprehend TCM texts (Lu, 2014). Similarly, vocabulary learning might also pose challenges to non-Chinese speaking learners even though some of them are English native speakers. In particular, there is a considerable number of loan words (e.g., qi) borrowed from Chinese in TCM given its origin. Knowing technical vocabulary is an essential element for learners in both settings mentioned above. 
To date, most English for Specific Purposes (ESP) vocabulary research in medical contexts has focused on Western medicine (e.g., Coxhead, 2018; Hsu, 2013; Lei \& Liu, 2016; Quero, 2015; Wang, Liang, \& Ge, 2008). However, as a previous student in a TCM Bachelor's degree, my observation of TCM lexis is that the vocabulary used in this specialized discipline is unique and that it is very different from the vocabulary used in Western medicine. TCM adopts a holistic approach to describing both health and diseases on the basis of the philosophy of Yin-Yang balance (Kaptchuk, 2001). In TCM, the patient's symptoms and signs need to be understood in relation to his/her entire being and behavior. That is, all things can always be seen as a part of a whole in this system of thought (Kaptchuk, 2001). In comparison, Western medicine places more emphasis on reductionism in that the symptoms and signs tend to be reduced to smaller parts or understood at the cellular and molecular levels though it pays more attention to the psychological and social dimensions of illness since the 1980s (Borrell-Carrio, Suchman, \& Epstein, 2004). For this reason, TCM and Western medicine have two different ways of perceiving the world of health, body, and illness, which is ultimately reflected in the language that describes it since language is central to the establishment of the science culture. Below is an example of a written TCM text to illustrate this point.

This condition is very chronic and occurs on a background of Spleen Yang deficiency and often Kidney Yang deficiency. In such cases one should add herbs to tonify Spleen and Kidney Yang such as Baizhu Rhizoma Atractylodis macrocephalae, Huangqi Radix Astragali membranacei, Yinyanghuo Herba Epimedii and Duzhong Cortex Eucommiae ulmoidis.

(TCM practice-based textbook sub-corpus)

This text first illustrates the use of Chinese-origin vocabulary in this field (e.g., yang, Huangqi). These words reflect the lexical and cultural origin of TCM, and have been labelled 'transliterated words' by Hsu (2016). Further, Hsu (2016) also reported that these words covered $10.45 \%$ of a TCM textbook corpus. However, little is reported by Hsu to help us find out more about these words in detail. The present research is thus to fill this gap by identifying and categorizing words of Chinese origin in English-medium TCM texts. 
Second, in TCM, basic words such as kidney and spleen have a very different meaning and set of references in comparison to their use in medical English. For example, kidney is the organ to store essence, govern birth, growth, reproduction and development, produce marrow, fill up the brain, control bones, house the will power and control the gate of life (Maciocia, 2005, p. 153). This example illustrates that the meaning of kidney has been extended in TCM, which is called metaphorization by Cognitive Linguists (e.g., Yu, 2003). According to Partington (1998), one key defining feature of discipline-specific lexis is that it tends to be rich in metaphoric uses. Such metaphors, however, might be semantically opaque to newcomers (Littlemore, Chen, Tang, Koester, \& Barnden, 2010) in that it may involve a considerable amount of figurative thinking (Littlemore \& Low, 2006) in order to obtain the metaphoric meaning of such lexis from their basic meaning. To better support TCM learners' specialized vocabulary development, it is important to know more about such features of the TCM vocabulary.

Third, the text above also illustrates the use of specialized vocabulary involving treatment (e.g., tonify), and Graeco-Latin vocabulary with specific meanings in TCM (e.g., Astragali membranacei is a herb which is used in TCM). These words are very unlikely to be met by the learners in their general English language use, regardless of whether English is their first language or second language. This suggests that the TCM learners are likely to need some help to deal with the technical vocabulary so as to function competently in their specialized discipline.

The aforementioned features of TCM vocabulary clearly demonstrate that TCM vocabulary is different from that of the Western medicine to some extent. Thus, there is a need to conduct research which examines the vocabulary used specifically in TCM. To the best of my knowledge, one study has made an attempt to develop a TCM-specific word list for learners in TCM with a textbook corpus of 4.48 million running words (Hsu, 2016). This TCM-specific word list includes lexical items that met the criteria of even dispersion, range (95 out of 100 textbooks), frequency (occurrence of 176 times) and specialized occurrence (beyond the most frequent 2,000 word families). Nonetheless, this study was only partially published as a paper in conference proceeding, and little has been documented about this list. As a result, the most important technical vocabulary in the discipline of TCM and its nature remain unknown. Further research to explore the TCM 
vocabulary, especially technical vocabulary, is justified. Importantly, no research has looked at the technical vocabulary in multiword units in the discipline of TCM. To achieve a comprehensive understanding of the nature of technical vocabulary, both single word and multiword units are explored in the present research.

Given the importance of TCM as an academic discipline and its availability in different countries, it has attracted students from different linguistic and cultural backgrounds. Those learners include Chinese first language speakers, English first language speakers, and even speakers of other languages such as Indonesian and Thai. These learners have different linguistic and cultural backgrounds, which are likely to influence their processing and comprehension of English-medium technical texts in their field. Earlier research revealed that learners face great challenges in understanding, learning, and utilizing technical vocabulary in their disciplines of study (Ardasheva \& Tretter, 2015; Berman \& Cheng, 2010; Evans \& Green, 2007; Evans \& Morrison, 2011; Gablasova, 2015; Mežek, 2013). Nonetheless, it is unclear what kinds of vocabulary pose most difficulty to the learners. Hence, it will be beneficial to investigate learners' understanding of technical vocabulary in their field of study to identify the kind of words that are especially challenging to different learners given their different backgrounds.

In addition, having a heavy load of specialized vocabulary is one of the prominent features of ESP more generally as reported by earlier research (e.g., Coxhead, Demecheleer, \& McLaughlin, 2016; Hsu, 2013; Nation, 2013). To better understand the lexical challenges faced by TCM learners, it is necessary to conduct a vocabulary load analysis of the study materials used by them.

\subsection{The aims of this thesis}

Given the lack of research into the lexis in TCM as a growing academic discipline and motivated by the vocabulary needs of learners in this discipline, research to explore the lexis in TCM is warranted. The present research sets out to address some of such research gaps with three aims. The first aim is a fundamental exploration of the kinds of words that make up the vocabulary used in the discipline of TCM. This involves a corpus-based study of the lexical demands of different types of English-medium TCM textbooks and journal articles so as to get an understanding of the lexis in the discourse domain of TCM. 
Such an understanding can serve as a foundational tenet for further research into the important words that the learners need to read the texts in their field, which is important for academic achievement.

The second aim of this research is to explore the nature of technical vocabulary in TCM. It involves the development of a pedagogically useful list of technical vocabulary of single word units for diverse learners in this discipline. Furthermore, a list of technical vocabulary of multiword units is also developed, as a supplement to the single word list. The multiword unit list is needed for two reasons. Firstly, some technical vocabulary needs to be seen in combinations rather than in single words (Ha, 2015; Ward, 2007). Secondly, research has shown that multiword constructions are very important in language use and learning (Ackermann \& Chen, 2013; Biber, 2009; Durrant \& MathewsAydınlı, 2011; Hyland, 2008; Hyland \& Tse, 2007; Liu, 2012). Therefore, technical vocabulary of multiword units can help us understand more about the technical nature of TCM texts and commonly used patterns of lexis in TCM. Consequently, this research explores technical vocabulary of both single and multiword units.

While there is little dispute that corpus-based word lists are important pedagogical resources, Martinez and Schmitt (2012) suggest that the difficulty of the lexical items for the learners should be taken into consideration to better facilitate the learning and teaching of technical words. The third aim of the present research is thus to explore the 'problematic' technical words in the technical word list mentioned above and find out what makes them challenging for the learners. It includes a qualitative investigation of 1 ) whether learners from different linguistic backgrounds have difficulty in the understanding of the technical vocabulary from the TCM technical word list, and 2) whether Chinese background learners and Western background learners understand the technical words in a same way given the different linguistic and cultural backgrounds they are from and the unique characteristics of technical vocabulary in TCM. As a result, this thesis may provide insights into the pedagogical merit by giving special attention to particular technical words in the list, taking into account learners' specific needs, lacunae in their knowledge, and their linguistic background. 


\subsection{The organization of this thesis}

The present thesis has eight chapters. This introductory chapter is followed by two literature review chapters (Chapter 2 and Chapter 3), which provide an overview of the general theoretical background that undergirds the current research. Chapter 2 describes the concept of technical vocabulary and its key properties. Chapter 3 looks into the relationship of technical vocabulary with general vocabulary and discuss word list studies, with a particular emphasis on specialized word lists.

Chapters 4, 5, and 6 address, in turn, each of the research aims described in Section 1.2, which are organized around three linked studies. The investigation begins with the lexical profile and vocabulary burden analysis of TCM texts using a corpus-based approach to develop a basic understanding of lexical characteristics and complexity in the study materials of TCM learners (Chapter 4). Based on such understanding, the next chapter (Chapter 5) focuses on more specific aspects of TCM vocabulary: the development of technical vocabulary lists of both single words and multiword units in the corpora with TCM textbooks and journal articles. Study 3 (Chapter 6) adopts a qualitative approach to explore learners' actual understanding of the technical words from the TCM single-word list developed from Study 2 to provide pedagogical insights into the learning aspects of technical vocabulary.

Chapter 7 discusses the main findings of the three studies in depth under four themes: (1) the importance of technical vocabulary; (2) learners' understanding of technical words; (3) discipline-specific word list development; and (4) the relation of TCM vocabulary to general vocabulary. Finally, Chapter 8 concludes the thesis by highlighting the theoretical, methodological and pedagogical contributions of this research. In addition, it also points out the limitations of the current research and directions for future research. 


\section{Chapter 2 Technical vocabulary}

\subsection{Introduction}

The present thesis investigates vocabulary, especially technical vocabulary in the specialist domain of Traditional Chinese Medicine (TCM) to support learners of this specialized discipline with vocabulary learning. This chapter introduces key concepts and significant studies relevant to the central focus of the thesis: technical vocabulary in TCM. It aims to clarify key concepts and discuss key issues in technical vocabulary research, to outline the properties of technical vocabulary, to indicate why technical vocabulary is so important in language teaching, learning, and research, and to look at technical vocabulary beyond single word units. While Chapter 2 discusses the properties of technical vocabulary, Chapter 3 looks at the place of technical vocabulary within the broader contexts of vocabulary learning and teaching, and word lists development as a way of helping learners to select or prioritize lexical items for study that will be most useful for their particular needs.

This chapter starts with an introduction of key terms and their definitions regarding technical vocabulary (Section 2.2). Section 2.3 discusses one key issue about technical vocabulary - its size. Section 2.4 reviews important approaches to identifying technical vocabulary. After that, Section 2.5 looks at aspects of knowledge involved in knowing a technical word. Then, the factors that might make technical vocabulary difficult to learn are examined (Section 2.6). Section 2.7 looks at another important aspect of technical vocabulary - technical vocabulary beyond single words. This chapter concludes with a brief summary of the discussion of technical vocabulary with research gaps identified from this part of literature which are to be addressed in the present thesis (Section 2.8).

\subsection{What is technical vocabulary?}

There are two ways in which technical vocabulary can be defined. The first, in a narrow sense, defines technical vocabulary as words which are "characterized by the absence of exact synonyms, resistance to semantic change, and a very narrow range" (Mudraya, 2006, pp. 238-239). For example, Monroe and Panchyshyn (1995) used this definition to describe technical vocabulary in mathematics (e.g., trapezoid, integer). 
The second definition broadly defines technical vocabulary as "those recognizably specific to a particular field" (Schmitt, 2010b, p. 77), ranging from items which are used exclusively by a particular field (pharmacology: agonist) to items which are used in general language but take on specialized meanings in a particular field (pharmacology: expression) (Nation, 2013; Schmitt, 2010b). This broad definition covers a broad range of lexical phenomena as shown in Table 2.1, which presents the kinds of words that constitute a technical vocabulary in pharmacology suggested by Fraser (2013).

Table 2.1: Components of technical words in pharmacology (Fraser, 2013)

\begin{tabular}{|l|l|l|}
\hline Labels & Components & Examples \\
\hline $\begin{array}{l}\text { Fully technical } \\
\text { vocabulary }\end{array}$ & $\begin{array}{l}\text { technical words almost exclusively used in } \\
\text { medical context }\end{array}$ & uconazole, sulphydril \\
\hline $\begin{array}{l}\text { Lay-technical } \\
\text { vocabulary }\end{array}$ & $\begin{array}{l}\text { technical words used both in general and } \\
\text { medical contexts with similar meanings }\end{array}$ & protein, therapy \\
\hline $\begin{array}{l}\text { Crytotechnical } \\
\text { vocabulary }\end{array}$ & $\begin{array}{l}\text { technical words taken on technical meanings } \\
\text { in medical context that differ from their } \\
\text { general meanings }\end{array}$ & $\begin{array}{l}\text { channel, sample, } \\
\text { medium }\end{array}$ \\
\hline
\end{tabular}

Interestingly, Fraser (2013) found that a very small number ( 6 out of 100 items) of the most frequent 100 headwords in a pharmacology word list were fully technical words which were only familiar to the subject specialists in pharmacology. A considerable number (19 out 100 items) of them were lay-technical words, whereas a large proportion (50 out of 100 items) of the words were crytotechnical words in familiar forms (e.g., expression, channel) but which had taken on very specialized meanings in medical contexts. Even though Fraser (2013) only examined 100 lexical items, it clearly illustrated the nature of technical vocabulary in pharmacology: while some technical words may appear only in one specialized field, others can be commonly met both in and outside a specialized area. All types of the technical words, nonetheless, are of equal importance to the creation and organization of subject knowledge. Since the present thesis focuses on exploring the nature of technical vocabulary in TCM, the broad definition of technical vocabulary is adopted. 
Similar to the definition of technical vocabulary, terminologies used to label technical vocabulary also varied substantially, including specialized vocabulary (Csomay \& Petrović, 2012), technical vocabulary (Chung \& Nation, 2003), sub-technical vocabulary (Yang, 1986), semi-technical vocabulary (Peters \& Fernández, 2013), technical terms (Pueyo \& Val, 1996; Yang, 1986), and jargon (Woodward-Kron, 2008). To avoid confusion, this thesis uses technical words to refer to technical vocabulary of single word units; while the term technical lexical bundles refers to technical vocabulary of multiword units (see Section 2.7). Technical vocabulary is used here as an umbrella term which embraces both technical words and technical lexical bundles.

\subsection{How to identify technical vocabulary in specialized texts?}

Having clarified the definition and terminology of technical vocabulary, this section discusses another important issue - how to identify technical vocabulary in specialized texts. It closely examines methods that have been used to identify technical vocabulary in different specialized fields.

\subsubsection{Corpus-based approach}

Corpus-based approaches have been widely used in technical vocabulary identification (e.g., Chung, 2003; Nazar, 2016; Periñán-Pascual, 2015; Peruzzo, 2014). Essentially, corpus-based approaches on technical vocabulary identification can be either linguistically-oriented or statistically-oriented. The linguistically-oriented sub-approach first searches and tags the formal linguistic and/or semantic features of the data in the corpora before submitting these features to various statistical analysis (e.g., Bertels \& Speelman, 2014; Kageura \& Umino, 1996; Peruzzo, 2014). In contrast, the statisticallyoriented sub-approach takes a straightforward approach by calculating and comparing the frequency and dispersion measures of lexical items (e.g., Chung, 2003; Nazar, 2016; Periñán-Pascual, 2015).

The corpus comparison approach is an example of such statistically-oriented subapproach (Chung, 2003) which is highly efficient. By comparing vocabulary in a specialized corpus and a comparison corpus, technical words which have unusual frequency in the specialized corpus can be identified. This approach uses the difference in the frequency of words between a technical corpus and a comparison corpus as the criterion on the basis that technical vocabulary occurs more frequently in specialized 
texts than in texts of general language use. A formula is often used to compare the relative occurrences of a word in the specialized corpus and the comparison corpus so as to decide which word can be considered as a technical word. For example, Chung (2003) used the following formula:

Ratio $=$ Normalized frequency of a word in the specialized corpus/Normalized frequency of the word in the comparison corpus

Normalized frequency is used because the size of the specialized corpus and the comparison corpus is unequal in Chung's (2003) study. Only words with a ratio of over 50 were selected as technical words.

Software programs like WordSmith Tools (Scott, 2012) (henceforth WordSmith) can automatically identify words with unusual frequency by computing the keyness of a word in the specialized corpus over a comparison corpus using the chi-square statistics with a $p$-value set at $\mathrm{p}<.000001$. The words which occur with a significantly higher frequency in the specialized corpus are called keywords and this method is called keyword analysis by Scott (1997). The keyword analysis has been applied in empirical studies to identify technical vocabulary (Ha \& Hyland, 2017; Mudraya, 2006).

Recently, more sophisticated statistical procedures and measures for technical vocabulary identification have been developed. Periñán-Pascual (2015) employed a composite measure that combines well-weighted values of a lexical item's salience, relevance, and cohesion (cohesion is used for identifying technical vocabulary of multiword units). In addition, Nazar (2016) used a comparative distributional analysis of the characteristics of the co-occurrence patterns of words in a technical corpus over a general corpus. The procedures and measures used in these recent studies have reportedly enhanced the accuracy of corpus-based approaches in the identification of technical vocabulary.

\subsubsection{Semantics-based approach}

Using the meaning of a word as criterion, Chung and Nation (2003, p. 105) proposed a four-step semantic rating scale to identify technical vocabulary in an anatomy text. The 
four-step rating scale was designed to measure how closely related the meaning of a word is to a particular specialized field. Step 1 and 2 contain words that have no relationship and are minimally related to the target discipline, respectively. Step 3 and 4 consist of words that are closely related to and specifically used in that target discipline. Only words rated at Step 3 or Step 4 were considered as technical words. The rating scale has been applied in empirical studies and has been proved to be quite reliable (Chung \& Nation, 2003; Fraser, 2005; Quero, 2015).

In addition to the semantic rating scale, a technical dictionary and the clues in the texts can also be used in the identification of technical vocabulary. Using a technical dictionary is relatively straightforward. If a word is found in a technical dictionary for that specialized domain, that word is regarded as a technical word (Chung \& Nation, 2004). However, the problem is that it is not always easy to find a reliable technical dictionary. Using contextual clues is quite laborious. It involves looking for clues in the specialized texts to determine whether a word is technical. The clues could take a variety of forms, for example, definition and labels in the diagrams (Chung \& Nation, 2004). Thus, using contextual clues is neither easy to apply nor efficient.

Another semantics-based approach is called systematic classification (Kwary, 2011). The systematic classification method is similar to what lexicographers do when they create a technical dictionary in consultation with a subject specialist who helps them select entries for the technical dictionary (Kwary, 2011). It involves identifying the general topics of the specialized field, and then selecting the terms that are relevant to each identified topic. One advantage of the systematic classification is that it allows multiword units and even symbolic vocabulary to be identified as technical vocabulary without limiting the number of words in each lexical term (Kwary, 2011). For example, $P / E$ stands for 'price-to-earning' in financial texts (Kwary, 2011), which could be identified by the systematic classification method but might be ignored by the computer-based corpus analysis because they might be regarded as letters of alphabet by the computer program.

To sum up, the semantics-based approaches of identifying technical vocabulary can take on different forms. They are generally very reliable, but are very time-consuming if every word in the corpus is checked. In addition, using the semantic rating scale requires good 
subject knowledge of that specialized area, especially the systematic classification which actually requires a subject expert to be involved.

\subsubsection{Mixed methods}

Recognizing the disadvantages of relying solely on a corpus-based approach or solely on a semantics-based approach, recent research on technical vocabulary tended to use a mixture of corpus-based and semantics-based approaches to identify technical vocabulary. Three studies have used mixed methods to identify technical vocabulary, which are to be reviewed in turn.

Kwary (2011) combined the keyword analysis and systematic classification to identify technical vocabulary, which was called the hybrid method. The keyword analysis identified words with unusual frequency in the technical corpus, which reduced the number of words to be examined in the systematic classification. The subsequent systematic classification helped to weed out any such mistakenly included items. Therefore, the hybrid method could help produce a comprehensive technical vocabulary list including single word units, multiword units, and symbolic vocabulary (Kwary, 2011).

Ha and Hyland (2017) combined keyword analysis with a semantics-based technicality analysis to identify technical vocabulary and to understand the nature of technical vocabulary in finance. The keyword analysis yielded a list of promising candidates which served as input for the self-proposed technicality analysis. Then, the three-stage technicality analysis was applied to categorize technical vocabulary into five groups according to their technicality, namely least technical, slightly technical, moderately technical, very technical, and most technical. For instance, fee is the least technical since it has the same sense in finance as its general use, while escrow is the most technical since it is unlikely to be known in general language. The technicality analysis sheds light on the nature of technical vocabulary in finance. However, it did not tell much about what the degree of technicality means to the learners or teachers.

In the medical context, Quero (2015) combined the corpus comparison method and the semantic rating scale of Chung and Nation (2004) to identify technical vocabulary in medical textbooks. The corpus comparison method was used to find out the potential 
technical words which have unusual frequency in the medical corpus; whereas the semantic rating scale was used to distinguish technical words from general words identified from the corpus comparison analysis. This study successfully illustrated that a combination of corpus comparison and semantic rating scale provided an efficient and effective way to identify technical vocabulary in medicine which contains a large number of technical words. Further information about the word lists developed from this study is provided in Section 3.3.2 about medical word lists.

Taken together, these three studies showed that a combination of a corpus-based approach and a semantics-based approach can efficiently and reliably identify technical vocabulary from specialized texts. The corpus-based approach and the semantics-based approach can compensate for the disadvantages of each other by taking advantage of the efficiency of the corpus-based approach and the accuracy of the semantics-based approach. These studies thus form the methodological basis of the present study.

\subsection{What is the coverage of technical vocabulary in specialized texts?}

While the previous section reviewed the methods to identify technical vocabulary, this part looks at another important issue of technical vocabulary - its coverage in specialized texts. That is, the proportion of technical vocabulary in specialized texts (Nation \& Waring, 1997).

Though it was estimated earlier that technical vocabulary only accounted for a small percentage of around $5.0 \%$ of the total tokens in specialized texts (Nation, 2001), one study from Chung and Nation (2003) has greatly broadened our ideas about what constitutes technical vocabulary and how much it covers technical texts. It was found that technical vocabulary constituted $31.2 \%$ of the total tokens in the anatomy texts and $20.6 \%$ in the applied linguistics texts (Chung \& Nation, 2003). Since then, Fraser (2013) also found that the most frequent 570 technical word families in pharmacology covered $26.7 \%$ of the tokens in the Pharmacology corpus. More recently, Quero (2015) found that technical vocabulary made up close to $37.0 \%$ of the tokens in the medical textbooks.

Together, the above studies show that technical vocabulary accounts for a large proportion of running words in specialized texts, which indicates that technical 
vocabulary plays a very important role in specialized texts. This is especially true in medical-related texts, in which almost every one out of three words is a technical word (Chung \& Nation, 2003). Such high density of technical vocabulary in medical texts highlights that technical vocabulary in that specialized domain deserves more attention. Moreover, considering the differences between Chinese and Western medicine, additional research on the technical vocabulary of TCM is needed.

\subsection{What is involved in knowing a technical word?}

In addition to needing knowledge of a large amount of technical vocabulary, an ESP learner also needs to know a lot about each individual technical item to fully understand that word and use it well in that specific context. According to Dudley-Evans and St John (1998), the teaching and learning of vocabulary in ESP followed similar principles to those applied to general vocabulary. Therefore, this section starts with the aspects of knowledge involved in knowing a word in general English and then moves on to the additional aspects of knowing a technical word.

\subsubsection{Knowing general aspects of vocabulary}

Vocabulary knowledge is a rich and complicated construct (Schmitt, 2010b). Although different researchers may have different ways to conceptualize vocabulary knowledge, it has been widely accepted that knowing a word is not just restricted to knowing the formmeaning relationship. Richards (1976) outlined that knowing a word involves knowing the spoken and written form, meaning(s), constraints on use, syntactic behavior, derivations, associations, and connotations of a word. Following Richards (1976), Nation (2013, p. 49) developed a comprehensive framework for describing vocabulary knowledge, including form (spoken, written, word parts), meaning (form and meaning, concept and referents, associations), and use (grammatical functions, collocations, constraints on use) as three aspects of knowing a word. Each aspect of knowledge in this framework includes both receptive and productive knowledge (see Nation, 2013, p. 27, for more details). Receptive vocabulary knowledge involves perceiving the form of a word through listening or reading, and retrieving its meaning; whereas productive vocabulary knowledge involves expressing its meaning though speaking and writing, and retrieving and producing the proper spoken or written form of the word (Nation, 2013). 
As mentioned above, knowing a word also involves knowing the associative relations of that word. Receptively, the learners should know what the target word makes them think of; while, productively, the learners should know what other words they could use instead of the target word (Nation, 2013). Recent research has shown that the word association responses can be used as a tool to investigate and understand ways in which the lexicon is structured and accessed in learners' mind (Fitzpatrick \& Izura, 2011). A similar rationale is applied to the present research to explore TCM learners' understanding of technical words.

In brief, Nation's (2013) framework has enabled us to conceptualize the aspects of word knowledge needed to know a general English word.

\subsubsection{Understanding the subject knowledge}

Knowing a technical word, however, involves more aspects than knowing a general word. According to Basturkmen (2006) and Douglas (2000), ESP language ability requires not only general linguistic knowledge and proficiency but also specific-purpose subject knowledge. The latter has been referred to by different labels: underlying competencies or underlying knowledge (Hutchinson \& Waters, 1985) and invisible discourse (Hirvela, 1997). Similarly, knowing a technical word involves not only the aspects of general vocabulary knowledge, but also understanding the word-related disciplinary concepts in that discipline. In addition, the learners need to develop ways of thinking and frames of reference in that specific discourse domain (Hirvela, 1997). To fully understand a technical word, learners need to know both different aspects involved in knowing a word generally and the underlying subject knowledge related to that word in a technical field.

In the field of TCM specifically, the specific-purpose subject knowledge involves, to a large extent, knowing the Chinese cultural background knowledge and the Chinese philosophy due to the medical tradition and culture from which TCM sprang. TCM has been developed over two millennia based on two core theories of Chinese philosophy - the theory of yin-yang and the theory of qi. Yin and yang originally mean the shady and sunny side of a slop, respectively (Kaptchuk, 2001). In Chinese philosophy, they always represent two opposite but complementary qualities, and all phenomena in the universe are the results of the interplay of two opposite stages, symbolized by yin and yang 
(Maciocia, 2005). Under this philosophical influence, the whole of TCM, its physiology, pathology, diagnosis and treatment could all be interpreted in terms of the essential and fundamental theory of yin and yang (Maciocia, 2005). Further, in Chinese culture, qi is the very basis of the universe's infinite manifestations of life, including minerals, vegetables, animals and human beings. (Maciocia, 2005). The changing nature of qi between a material substance and an ethereal is central to the TCM view of body and mind as an integrated unit (Maciocia, 2005). Thus, the TCM expert - Kaptchuk (2001) pointed out that TCM learners need to be familiar with Chinese philosophy and culture to understand TCM as it is influenced by these philosophies.

The intricate relationship between TCM and Chinese culture has been illustrated in a study about culture and language by $\mathrm{Hu}$ and Fong (2010). They found that Chinesespeaking learners of English have problems understanding idioms associated with heart and mind because Chinese and English culture have different conceptions of these words. In English culture, there is considerable overlap between head and mind with mind located in the head region; whereas the concept of mind tends to overlap more with heart than head in Chinese culture (Hu \& Fong, 2010). This overlap can be traced back to the theory of internal organs of TCM, in which heart is defined as the organ that governs mind (Maciocia, 2005). Such cross-cultural differences between Chinese and Western conceptions of body and health led to different ways in which these words were construed in different languages. Therefore, when Chinese-background learners interpreted the idiom speak one's mind (say something without reservation), they tended to interpret it as being honest and true to someone, which is similar to zhen xin (true heart) in Chinese. This interpretation is understandable given the close relationship between mind and heart in Chinese culture.

The study of Hu and Fong (2010) has two important implications. First, this study showed that TCM and Chinese culture are integrated. To understand technical vocabulary in TCM, the learners need to understand the underlying Chinese culture and philosophy as part of the subject knowledge, and develop ways of thinking and frames of reference in this culture. Second, it suggests that TCM learners may experience challenges in those places where the target language construed things differently from their native language as a result of cross-cultural differences (e.g., mind and heart). Given the cultural differences 
between Chinese and Western conceptions of body and health, TCM and Western medicine have two different ways of seeing health and illness, which means they represents two different ways of thinking (Kaptchuk, 2001). Such difference is ultimately reflected in the language that describes it though the difference between the two types of medicine is larger than the descriptive language. It is possible that some technical terms in TCM, either borrowed intact from Chinese or substituted by an English-word translation, activate a radial network of associations in Western-background TCM students that only partially overlaps with that of Chinese-background students. Therefore, it is interesting to explore whether Chinese and Western background learners understand the technical words in a same way given the different linguistic and cultural background they are from.

\subsubsection{Summary}

This section has shown that knowing a technical word not only involves knowing general aspects of vocabulary knowledge, but also understanding the underlying subject knowledge as an additional aspect of knowledge. For TCM learners specifically, knowing a technical word also involves knowing the Chinese culture and philosophy underlying the subject knowledge. Previous research has shown that language learners found it challenging to construe things in the target language which are construed differently from their native language as a result of cross-cultural differences. As a consequence, further research on technical vocabulary in TCM should consider the variation in learners' cultural background to better support learners' technical vocabulary development.

\subsection{What might make a technical word difficult to learn?}

Technical vocabulary is perceived by practitioners and learners as one of the biggest challenges impeding both the teaching and the learning of the subject knowledge in ESP (Ardasheva \& Tretter, 2015; Tiersma, 1999). This section looks closely into the factors that might make a technical word challenging to learn. It begins with a brief summary of the different linguistic features that affect the difficulty of a word proposed by previous research. It then discusses the features on which this thesis focuses.

To begin with, it might be useful to look at a seminal paper of Laufer (1997), who listed several linguistic features inherent in the word itself that could affect the ease or difficulty to learn that word. These features are pronounceability, orthography, length, 
morphology, part of speech, synformy, abstractness and specificity, and semantic features (Laufer, 1997, pp. 142-153).

Among the different linguistic features, semantic features are of particular interest to the ESP researchers regarding technical vocabulary. They include multiplicity of meanings and semantic extension and transfer such as metaphorization. The fact that a substantial number of technical words have multiple meanings has long been noticed and it has been regarded as one important factor that impedes the comprehension of technical words (Csomay \& Petrović, 2012; Fraser, 2007, 2009, 2012, 2013; Nation, 2013; Nation, Coxhead, Chung, \& Quero, 2016; Schmitt, 2010b; Tiersma, 1999; Watson Todd, 2017). Multiplicity of meaning can either be polysemy or homonymy depending on whether the multiple meanings of the word form are related (Laufer, 1997). An example of polysemy is failure in medicine, which means an organ stops working (e.g., heart failure) whereas its general meaning is lack of success. An example of homonymy is briefin law. It is a noun in law which refers to "a type of legal document" (Tiersma, 1999, p. 111) instead of being an adjective as in brief description. Empirical research evidence has shown that multiple meanings of a word, be it polysemy or homonymy, impedes learners' comprehension of that word (Laufer, 1997). Hence, it is also regarded as one of the greatest challenges in technical vocabulary learning by Fraser (2012).

Metaphorization is known as the transfer and extension of the meaning of words as a result of the interaction between language and thought (Charteris-Black, 2000). It is one of the major mechanisms that create polysemy. As pointed out by Partington (1998), one key defining feature of discipline-specific lexis is that that it tends to be rich in metaphoric uses. For instance, when business people talk about ring-fencing, they mean limiting budgetary liability (Littlemore et al., 2010, p. 192). As was quantified by Low, Littlemore, and Koester (2007), the metaphoric density in academic lectures ranges from $10 \%$ to $13 \%$ of all lexical items. Such metaphors might be semantically opaque to newcomers when they first enter a field (Littlemore et al., 2010) because it may involve a considerable amount of figurative thinking (Littlemore \& Low, 2006) in order to obtain the metaphorical meaning from the basic meaning of the words. 
In the field of TCM, the metaphorical use of language has also been identified in a study of metaphor, body and culture by $\mathrm{Yu}$ (2003). Yu showed how the abstract concept courage was related to gallbladder in 'gallbladder is container of courage' via a conceptual metaphor grounded on the TCM theory of internal organs. In TCM theory, the gallbladder has two main functions. First, it stores and excretes bile that is secreted by the liver. The second function of the gallbladder, which is probably unique to TCM, is that the gallbladder has the capacity to make decisions in mental processes and activities, and it also gives people courage (Maciocia, 2005). Gallbladder thus has an extended meaning related to courage in Chinese culture. 'Gallbladder is the container of courage' is a culturespecific metaphor shaped by the underlying TCM theory (Yu, 2003). This study revealed that the meaning of the words for organs, such as gallbladder, is often extended because of their unique functions in TCM. Yu (2003) called this process metaphorization. Further, $\mathrm{Yu}$ (2003) pointed out that metaphor is actually the bridge that connects the human body and the outside universe in TCM influenced by the views of Chinese philosophy. As a result, this study demonstrated that the TCM technical words might be difficult to understand because they might be familiar words which contain cultural metaphors as in the case of gallbladder.

With regard to specificity, Laufer (1997) pointed out that the more specialized a word is, the more difficult it is to learn that word. In other words, the words that are used in a wider variety of contexts are easier to learn than words that are restricted to narrower contexts of use. The same principle can be applied to technical words within a specialized field as demonstrated by Fraser (2012). He argued that words used in all sub-disciplines of pharmacology (e.g., receptor, channel) tended to be easier to learn than words used with higher specificity such as toxicosis which is used specifically in toxicology. Accordingly, fully technical words might be difficult for most learners because they rarely occur frequently in general language or even occur exclusively in one field, indicating that there are few opportunities to encounter them before entering this field. Similarity of lexical forms, or synforms, is another potential area of difficulty in vocabulary learning (Laufer, 1997). The term synforms refers to pairs of words which sound alike or look similar or both. For instance, mediate and medicate are a pair of synforms found in Pharmacology by Fraser (2012). 
To sum up, this section has shown that there are many factors that might make a word difficult to learn. Nonetheless, these are merely 'potential' difficulties as pointed out by Fraser (2012) since few empirical studies have investigated the difficulties that learners actually have in understanding technical vocabulary. Thus, further research to investigate what actually affects the ease or difficulty of technical words is warranted. Such research will provide better insights into the factors that make a technical word easy or hard, and thus give some indication of how technical vocabulary can be prioritized for teaching with the limited course time available.

\subsection{Technical vocabulary beyond single word units: Lexical bundles}

Previous research has shown that multiword expressions are crucially important in language use and learning (Ackermann \& Chen, 2013; Biber, 2006, 2009; Durrant \& Mathews-Aydınlı, 2011; Hyland, 2008; Hyland \& Tse, 2007; Liu, 2012). First, multiword expressions are ubiquitous in language use overall (Biber, Johansson, Leech, Conrad, \& Finegan, 1999; Nattinger \& DeCarrico, 1992; Ward, 2007). Second, such multiword expressions can effectively help language users increase fluency (Arnon \& Snider, 2010; Ellis, Simpson-Vlach, \& Maynard, 2008; Simpson-Vlach \& Ellis, 2010). Additionally, Gardner (2013, p. 80) has pointed out the potential cost of focusing on single words only in a case study. He found out that important meanings were disregarded when organ system, nervous system, circulatory system, immune system, reproductive system, excretory system, lymphatic system, skeletal system, and respiratory system became simply system with a high frequency count on a list of core single words. Furthermore, Fraser (2012) pointed out that technical multiword expressions in the specialized texts help us understand the technicality of its components through the way in which these words combine and interact with other words. For the above reasons, multiword units in the technical texts, especially technical multiword units, deserve considerable attention from learners, teachers and researchers.

Despite that importance, research on technical lexical bundles remains fairly limited in vocabulary research. Among the studies which investigated lexical bundles in academic disciplines, the majority focused on the most frequent lexical bundles shared by a variety of disciplines (Ackermann \& Chen, 2013; Ädel \& Erman, 2012; Byrd \& Coxhead, 2010; Hsu, 2014b; Hyland, 2008; Liu, 2012). Technical lexical bundles, however, is an 
underexplored field of multiword studies. This section will first look at the term technical lexical bundles - that is used to describe technical vocabulary beyond single word units in this study. Then, it examines approaches to identify lexical bundles, especially in academic contexts. The last subsection looks at the very few studies which have investigated lexical bundles in specialized discourse.

\subsubsection{What are 'lexical bundles'?}

The term lexical bundles is used in the current research to describe multiword items beyond single word units, covering all types and lengths of multiword items (e.g., collocations, and phrasal/prepositional verbs). As defined by Biber et al. (1999, p. 990), "lexical bundles are recurrent expressions, regardless of their idiomaticity, and regardless of their structural status". That said, to make their investigation manageable, Biber et al. (1999) restricted lexical bundles to recurring sequences of three or more words. Another reason for this decision was that Biber et al. (1999) argued that shorter bundles were often incorporated into longer lexical bundles. While this definition suited the aim of the present thesis about multiword expressions, the present thesis extended the scope of lexical bundles to two to five words in length. This decision was made because the present thesis focused on only one register - written discourse of TCM. It was rather narrowly focused compared to the large-scale investigation of written and spoken English across a wide range of disciplines. In addition to lexical bundles, other labels are also used in studies of multiword units, including formulaic sequences (Hsu, 2014b; Wray, 2000), formulas (Simpson-Vlach \& Ellis, 2010), word clusters (Carter \& McCarthy, 2006). In order to avoid confusion, the term lexical bundles is used in this research, and thus the term technical lexical bundles is used to refer to technical vocabulary beyond single word units.

\subsubsection{How to identify lexical bundles in the specialized texts?}

Using corpora to identify lexical bundles is probably the most widely used and efficient approach. Corpora with a large collection of texts can be used to automatically extract lexical bundles occurring more than random probability with the assistance of computer programs (Ackermann \& Chen, 2013; Biber, 2009; Hyland, 2008; Liu, 2012). In such corpus-based studies, frequency is one of the most important selection criterion in the identification of lexical bundles similar to the study of single word units. The study by Biber, Conrad, and Cortes (2004) of lexical bundles in university teaching and textbooks 
is a typical example which relied largely on frequency to identify lexical bundles. Those high-frequency bundles were then studied in terms of the function they served in the academic spoken and written texts. This approach was also called form-first approach by Durrant and Mathews-Aydinlı (2011), and it has been commonly used by researchers (e.g., Ädel \& Erman, 2012; Biber, 2009; Byrd \& Coxhead, 2010; Cortes, 2004; Hyland, 2008; Simpson-Vlach \& Ellis, 2010).

However, Durrant and Mathews-Aydınlı (2011) pointed out that this form-first approach had a limitation from the pedagogical perspective. It is the functions of the lexical bundles rather than the frequency information that matter for the learners. Based on the view that functions of the lexical bundles are of the primary importance, they used a functionfirst approach to study lexical bundles in student essays and research articles. Specifically, they first annotated the target corpora for communicative functions, then identified the recurrent lexical bundles associated with each function. For example, will be followed is the formula used to signal position. Nonetheless, it should be acknowledged that this approach was labor intensive.

In sum, while the form-first approach is a bottom-up approach, the function-first approach is a top-down approach. Both approaches provide valuable insights into the nature of lexical bundles in academic discourse. The form-first approach helps us effectively explore what lexical bundles are frequently used in a selected corpus or area, whereas the function-first approach could help us select lexical bundles which are of practical use to the teachers and learners. Both approaches have their limitations as mentioned above. However, both approaches are valuable to the present research since they provide a methodological basis for the study of technical lexical bundles. To choose a suitable approach for lexical bundles studies, the research aim should be taken into account.

\subsubsection{Lexical bundle studies in disciplinary contexts}

Having reviewed the approaches used to identify lexical bundles, this section looks at recent studies on lexical bundles in different disciplines which illustrated the importance of technical lexical bundles. Five studies (Ha, 2015; Nazar, 2016; Periñán-Pascual, 2015; Ward, 2007; Yang, 1986) have investigated such technical vocabulary beyond single 
word units. This section focuses on two of them, which are important to the present research.

Ward (2007) examined two/three/four-word technical collocations in engineering textbooks. Looking at the vocabulary in two engineering corpora - one with chemical engineering textbooks, and the other one with textbooks from five engineering subdisciplines, Ward (2007) found that there is a divergence in the most common nouns used in different engineering sub-disciplines. Then, he examined the collocational behavior of three common nouns (gas, heat, liquid) in chemical engineering textbooks, and found that these nouns typically appeared in noun phrases (or collocations). This finding suggested that there is a widespread use of technical collocations in specialized discourse and thus they deserve more attention. Ward (2007) also examined the abovementioned collocations in other sub-disciplines, and found that those collocations from the chemical engineering occurred seldom in other sub-disciplines. Therefore, this study suggested that collocations can be sub-discipline specific. Nonetheless, the small corpora used in this study renders this claim tentative. Further, Ward's (2007) results also revealed that the creation of new collocations often represents an early stage of specialization, which can be seen as the threshold to specialized engineering discourse. This finding highlighted the importance of technical vocabulary beyond the single word units for the creation of subject knowledge.

Noticing that studies about technical lexical bundles were very limited, Ha (2015) proposed a categorization method to assess the technicality of two to four-word lexical bundles and categorize the technical lexical bundles. She identified 539 technical lexical bundles from a Financial Corpus of 6,753,212 tokens based on frequency, range and meaning criteria. Depending on whether the meaning of the bundles can be directly derived from the literal meaning of its components, Ha (2015) categorized them into visible/least (e.g., mortgage loans, financial statements) or synergistic technical bundles (e.g., real estate, fair value). Those technical lexical bundles whose meaning cannot be directly derived from the literal senses of its constituents were synergistic technical lexical bundles, otherwise they were visible or least technical bundles. The visible technical lexical bundles were distinguished from least technical lexical bundles on the basis that only one component in the bundles was a technical word. The synergistic 
technical lexical bundles are an interesting linguistic phenomenon since they illustrated how the technical knowledge can be lexicalized through the combination of technical words or/and non-technical words. They thus improve our understanding of the nature of technical vocabulary in finance in a way that the individual technical words cannot. Such synergistic lexical bundles deserve more attention since they might be semantically opaque to the learners. However, the distinction between visible and least technical bundles seems to have limited pedagogical value.

Taken together, these two studies have three important implications. First, they suggest that lexical bundles are ubiquitous in language use, and thus they are indispensable components in vocabulary learning and teaching. Further, it was suggested that lexical bundles are largely discipline-specific. Third, technical lexical bundles can help us understand the nature of technical vocabulary in the specialized areas in a way that the individual technical words cannot. As a result, further research about technical lexical bundles is needed. Such research of multiword units can be useful to enhance the learners' knowledge of technical single words. Considering this, further research into technical vocabulary should take lexical bundles into account.

\subsection{Summary}

This section has looked at technical vocabulary from different perspectives, including the definition, identification, coverage, components of technical vocabulary, knowledge involved in knowing a technical word, potential difficulty of learning technical vocabulary, and technical vocabulary beyond single word units. It sheds light on the nature of technical vocabulary and highlights the importance of technical vocabulary of both single and multiword units. At the same time, it also indicates the potential difficulty of learning technical vocabulary.

Based on the review of literature in the present chapter, three major gaps have been identified. The first concerns vocabulary research in the discipline of TCM. While medical vocabulary has been extensively investigated, little attention has been paid to vocabulary in TCM even though TCM is growing as an important discipline at universities across different countries. The only study that has looked at vocabulary in TCM till the time when this research was conducted is Lu and Durrant (2017), which investigated the 
usefulness of the AWL and AVL in TCM research articles. Considering the differences between the Western medicine and TCM, the findings about the vocabulary in Western medicine are unlikely to be generalizable to TCM. Thus, it would be beneficial to closely examine the lexical characteristics in TCM to achieve a comprehensive understanding of vocabulary in this specialized discipline before proceeding to further detailed investigation.

The second gap relates to technical vocabulary, especially technical vocabulary in TCM. Previous research indicated that while technical vocabulary is essential to the creation of specialized knowledge and accounts for a large proportion in the specialized texts, it still remains an underexplored area. Moreover, less is known about what kind of vocabulary constitutes a technical vocabulary in TCM given that TCM is embedded with Chinese culture and philosophy due to its Chinese origin.

Third, the literature shows that lexical bundles have always been of some interest to language teachers and learners who work in academic discourse community. The last two decades have witnessed a concerted focus on general and academic lexical bundles in academic writing and speech motivated by the idea that lexical bundles help to promote fluency. In contrast, research about technical lexical bundles is rare. To better understand the technical vocabulary in a field, it is necessary to look at the technical lexical bundles in addition to the technical vocabulary at the level of single words.

As a result, it was suggested that more research on technical vocabulary is needed especially in those underexplored disciplines such as TCM. Further, little is known about the actual difficulty that the learners meet in learning and understanding technical vocabulary. Such research into technical vocabulary can provide a better insight into the difficulty of learning technical vocabulary and thus provide a more solid basis for proposing guidelines for teaching. In the next chapter, how technical vocabulary is situated in the broader context of vocabulary learning and teaching will be discussed. In addition, it also reviews studies related to how word lists can be developed to assist vocabulary learning and teaching. 


\section{Chapter 3 Word lists and vocabulary learning}

\subsection{Introduction}

Chapter 2 has discussed the properties of technical vocabulary. This chapter looks at technical vocabulary from two other angles. First, it looks at the place of technical vocabulary within the broader contexts of vocabulary teaching and learning. Then, it examines word list studies, especially discipline-specific word list studies, in relation to vocabulary learning and teaching in general. The last aim of this chapter is to look at how well-constructed word lists can be used to help us understand the lexical characteristics of a given text.

Section 3.2 looks at development and use of word lists as an important instrument of vocabulary learning and teaching in English language teaching history. Section 3.3 reviews the classification of vocabulary, from where we can see how technical vocabulary is situated in the broader context of vocabulary learning and teaching. Section 3.4 reviews research which makes use of well-constructed general word lists to conduct vocabulary profile analysis as a way to understand the vocabulary characteristics of given texts. This chapter concludes with Section 3.5, which summarizes the literature review with research gaps identified and rationale for the first study of the present research.

\subsection{Where is technical vocabulary situated in vocabulary learning?}

Having recognized the importance of technical vocabulary, this section looks at the place of technical vocabulary within the broader context of vocabulary research in relation to English for academic purposes (EAP) and English for specific purposes (ESP). In recent vocabulary research, technical vocabulary has been portrayed in different vocabulary frameworks. The first framework is the four-part categorization while the second is the frequency-based classification (Nation, 2013; Schmitt \& Schmitt, 2014). These frameworks are introduced in turn in the following paragraphs.

Using learning purposes as the criterion, vocabulary can generally be classified into four parts: general high-frequency vocabulary, academic vocabulary, technical vocabulary and low-frequency vocabulary (Nation, 2001). General high-frequency vocabulary (e.g., the, happy) refers to the most frequently used words in English which are very important 
in both spoken and written English. In contrast, low-frequency vocabulary refers to vocabulary that occurs rarely in English. Academic vocabulary (e.g., sustainability, concept) refers to a set of lexical items that occur frequently in a wide range of academic areas but do not occur frequently in non-academic areas (Nation, 2001). Technical vocabulary refers to words which are closely related to the subject area. Such words are used frequently in that subject area but may not be frequently used in other areas. For instance, accretable and capital are technical words in finance but are not technical words in medicine.

Recognizing that academic/technical vocabulary did not fill the gap between highfrequency and low-frequency vocabulary and inspired by Nation's (2006) frequencybased descriptions of English vocabulary, Schmitt and Schmitt (2014) restructured the vocabulary classification framework by adding a new category, one of mid-frequency vocabulary between high-frequency and low-frequency vocabulary. In this frequencybased classification of vocabulary, high-frequency vocabulary (e.g., of, forest) was defined as the most frequently used 3,000 word families of English, while low-frequency vocabulary (e.g., spectrometry, adenomatous) was defined as those beyond the most frequent 9,000 word families. Mid-frequency vocabulary (e.g., academic, hypothesis) thus included the word families between high and low-frequency vocabulary, namely the most frequent 4,000 to 8,000 word families.

This frequency-based classification of vocabulary was also used by Nation (2013). Since then, academic and technical vocabulary were put under the category of specialized vocabulary, which could cut across high, mid, and low-frequency vocabulary levels (Nation, 2013; Nation et al., 2016). It is important to note that, in this classification, the definition of high-frequency vocabulary was different from that of the four-part classification, which defined the most frequent 2,000 word families as high-frequency vocabulary (Nation, 2013; West, 1953). Schmitt and Schmitt (2014) argued that 3,000 word families are the most appropriate cut-off point of high-frequency English vocabulary after reviewing a number of frequency and acquisition studies and exploring the amount of vocabulary needed for English usage, the range of graded readers and dictionary defining vocabulary. This classification was used in this thesis to define high, mid and low-frequency vocabulary because 3,000-word-family level fits medical students' 
proficiency level according to Hsu's (2013) estimate of medical students' vocabulary size in Taiwanese context. The first-year medical students are expected to have a vocabulary size of 3,000 word families since those students need to perform optimally in different disciplines including the English subject to be accepted by a medical university.

This section has provided a brief overview of the place where technical vocabulary is situated in the broader context of vocabulary learning and teaching. This overview demonstrates how technical vocabulary is related to academic vocabulary, and also how it is related to high, mid and low-frequency vocabulary in different categorization frameworks. The academic/technical classification of vocabulary is in line with the classification between EAP and ESP approaches within the sphere of English language teaching. The EAP approach focuses on the teaching of the linguistic features and core skills that are common to all academic disciplines, while the ESP approach emphasizes the teaching of linguistic features that are different from one specific discipline to the other (Dudley-Evans \& St John, 1998). The present thesis focuses on technical vocabulary in the discipline of TCM, thus it relates closely to the ESP approach from an English language teaching perspective.

\subsection{Developing and using word lists}

Due to the fact that English has a huge vocabulary (Schmitt, 2010b), a recurring issue in English teaching research has been that of how vocabulary can be organized for learning and teaching. Creating frequency-based word lists has been an effective and widely used approach to address this issue. The central insight of the frequency-based word lists is that words in a language do not occur with similar frequencies: some words occur only once in a whole text while some words occur almost in every sentence (Nation et al., 2016). In other words, some words are likely to be more important than the others as they appear frequently in the target language. Frequency information thus provided a rational basis for the learning efforts by ensuring that words studied will be met often in their language use (Nation \& Waring, 1997). By prioritizing lexical items with high frequency, the learners can have the greatest possible communicative success with the inevitably limited set of words they learn (Durrant, 2013). Similarly, frequency-based word lists developed from a corpus of texts representing language uses of a specific 
discourse community can provide valuable information about how these words are used in that discourse community (Biber, Conrad, \& Reppen, 1994).

This section discusses previous studies on word list development with a focus on discipline-specific word lists. To begin with, it discusses the units for counting a word as this is one core research issue of recent lexical studies, especially word list studies (Section 3.3.1). Then, it briefly reviews general high-frequency word lists for any learning purposes (Section 3.3.2) and generic academic word lists created for a wide range of academic disciplines (Section 3.3.3). This is because general vocabulary lists and generic academic word lists lay the foundation for the development of discipline-specific word lists. Further, the methods used to develop general word lists and generic academic word lists are important for the development of discipline-specific word lists. After that, it looks at the frequency-based word lists created specifically for one specialized discipline (Section 3.3.4) and medicine-specific word lists (Section 3.3.5).

\subsubsection{The essential units of counting in word list studies}

There are five commonly used units for counting words in a text or corpus: tokens, word types, lemmas, flemmas, and word families. The term tokens refers to the number of running words occurring in a text or corpus (Nation, 2013). Repeated word forms are counted as separate tokens. Token is the simplest and smallest unit for counting words. Word type refers to the number of unrepeated word forms occurring in a text (Nation, 2013). For example, in the previous sentence (excluding reference), there were 14 tokens but only 13 word types since word occurred twice as italicized. Previous specialized vocabulary studies have shown that word type was the most sensible unit of counting specialized vocabulary (e.g., Coxhead et al., 2016; Ha, 2015; Khani \& Tazik, 2013; Quero, 2015; Ward, 2009; Watson Todd, 2017). However, it should be noted that using word types as the unit in word list development means items such as point and points appear as two items in a word list. This may not be helpful considering that even low-level learners may have the morphological knowledge to tell points is the plural form of point.

Lemma is "a set of lexical forms having the same stem and belonging to the same major word class, differing only in inflection and/or spelling" (Francis \& Kučera, 1982, p. 1). For example, study (verb) and its inflectional forms (studying, studies, studied) make up a 
lemma. However, study (noun) is counted as another lemma. Gardner and Davies (2014) argued that lemma is a sensible unit for counting to develop pedagogical word lists. The word lists with lemma as unit for counting can be more informative than word-family lists since lemmas take the grammatical functions of lexical items into account. As a result, lemma has been more common as unit of counting in word list studies about general vocabulary and specialized vocabulary (e.g., Brezina \& Gablasova, 2015; Gardner \& Davies, 2014; Lei \& Liu, 2016). A more recent unit for counting words is flemma, which is an extended version of lemma that does not distinguish between parts of speech (Pinchbeck, 2014). In other words, flemma includes words of different parts of speech in the same lemma. In this regard, study (verb) and study (noun) are counted as one flemma. It has been used as unit of counting in a number of word lists studies for beginners (e.g., Browne, 2014; Dang \& Webb, 2016) because flemma is the most appropriate unit for counting which takes beginners' limited morphological knowledge into account. However, lemma and flemma may not be suitable units for counting in technical vocabulary studies as not all members of a lemma or flemma are technical words (Chung \& Nation, 2004; Nation, 2016b). For example, tree (means hierarchy) is a technical word in engineering while trees is not.

The largest unit of counting is word family, which consists of a stem word with its inflectional forms and transparent derivational forms (Nation, 2013). As to what derivational forms should be included in a word family, Nation (2012) determined that it should be derivational forms in a word family from Level 3 to 6 in Bauer and Nation's (1993) hierarchical scale of inflectional and derivational affixes as can be seen from Table 3.1.

Table 3.1 presents Bauer and Nation's (1993) 7 levels of word family with affixes at each word family level. It should be pointed out that, if a word family is defined as being at a particular level, it includes the stem word together with its inflections and derivations made up of all affixes up to that level. Nation (2012) included derivational forms from Level 3 to 6 in a word family, which means he adopted Bauer and Nation's (1993) Level 6 of word family. In other words, a word family can potentially include the word stem itself (Level 1) together with its inflections (Level 2) and derivations made up of all affixes from Level 3 to Level 6. 
Table 3.1: Bauer and Nation's (1993, p. 257-p. 262) word family levels

\begin{tabular}{|c|c|c|}
\hline Level & Affixes & Example \\
\hline Level 1 & Every form is a different word. & imagine \\
\hline Level 2 & $\begin{array}{l}\text { Inflectional suffixes (plural, third person singular, present } \\
\text { tense, past tense, past participle, -ing, comparative, } \\
\text { superlative, and possessive) }\end{array}$ & $\begin{array}{l}\text { imagining, } \\
\text { imagined, } \\
\text { imagines }\end{array}$ \\
\hline Level 3 & $\begin{array}{l}\text { Most frequent and regular derivational affixes (-able, -er, } \\
\text {-ish, -less, -ly, -ness, -th, -y, non-, and un- with restricted } \\
\text { uses) }\end{array}$ & $\begin{array}{l}\text { imaginable, } \\
\text { imaginably }\end{array}$ \\
\hline Level 4 & $\begin{array}{l}\text { Frequent and orthographically regular affixes (-al, -ation, } \\
\text {-ess, -ful, -ism, -ist, -ity, -ize, -ment, -ous, and in- with } \\
\text { restricted uses) }\end{array}$ & $\begin{array}{l}\text { imagination, } \\
\text { imaginations }\end{array}$ \\
\hline Level 5 & $\begin{array}{l}\text { Regular but infrequent affixes (-age, -al, -an, -ance, -ant, - } \\
\text { ary, -atory, -dom, -eer, -en, -ence, -ent, -ery, -ese, -esque, - } \\
\text { ette, -hood, -i, -ian, -ite, -let, -ling, -ly, -most, -ory, -ship, - } \\
\text { ward, -ways, -wise, -ante, anti-, arch-, bi-, circum-, -counter, } \\
\text {-en, -ex, fore-, hyper-, inter-, mid-, mis-, neo-, post-, pro-, } \\
\text { semi-, sub-, un-) }\end{array}$ & imaginary \\
\hline Level 6 & $\begin{array}{l}\text { Frequent but irregular affixes (-able, -ee, -ic, -ify, -ion, -ist, } \\
\text {-ition, -ive, -th, -y, -pre, re-) }\end{array}$ & $\begin{array}{l}\text { imaginative, } \\
\text { imaginatively }\end{array}$ \\
\hline Level 7 & $\begin{array}{l}\text { Classic roots and affixes (e.g., } a b-, a d-, c o m-, d e-, d i s-, e x-\text {, } \\
s u b-\text { ) }\end{array}$ & Not applicable \\
\hline
\end{tabular}

The third column in Table 3.1 provides such an example of word family with imagine and its family members. However, the notion of word family is still problematic. As shown in Table 3.1, -able is an affix which can be found both at Level 3 and Level 6 . This is because the transparent cases are dealt with at Level 3 (e.g., develop - developable) while the opaque items are dealt with at Level 6 (e.g., attenuate - attenuable) (Bauer \& Nation, 1993). Therefore, word list developers should be cautious when using word family as unit of counting. Nevertheless, word families have been extensively used as a unit of counting in various word list studies (e.g., Coxhead, 2000; Hsu, 2013; Mungra \& Canziani, 2013; Nation, 2012; Valipouri \& Nassaji, 2013; Wang et al., 2008; West, 1953; Xue \& Nation, 1984; Yang, 2015) because it is a sensible unit of counting words if one has a learner's 
receptive use of the language in mind. That is because it has been assumed that knowledge of one member of a word family will help a learner work out the meaning of other members of that family. Nonetheless, the use of word family as the unit of counting has been questioned because EFL learners may not have sufficient morphological knowledge to see the relationship between a base word and its inflections and derivations (Kremmel, 2016; Schmitt \& Zimmerman, 2002; Ward \& Chuenjundaeng, 2009).

Having clarified the concepts of token, type, lemma, flemma and word family, and having introduced Bauer and Nation's (1993) scale of word families, it is useful to connect them so that these units of counting can be comparable. As pointed out by Nation et al. (2016), word type, lemma, flemma, and word family can actually all be placed in Bauer and Nation's (1993) Level 7 scale. Specifically, a word type can, in fact, be placed at Level 1, whereas lemma is at Level 2 and flemma is at Level 2.5 in the scale. Further, a word family is at Level 6 of Bauer and Nation's (1993) scale as defined by Nation (2012). Of particular concern here is the question of what unit to use in word list studies. This however is not a simple question. In general, it is suggested that the unit of counting words should match the purpose of research, the characteristics of the target learners and the resource available in that research (Dang, 2017; Nation, 2013; Nation et al., 2016; Schmitt, 2010a). This thesis focuses on investigating the nature of TCM vocabulary and developing a technical word list for TCM learners, choosing an appropriate unit of counting words is of essential importance. The choice of unit of counting will be clarified in the description of each study with justification provided.

\subsubsection{General high-frequency word lists}

It is commonly assumed that learners already have knowledge of general high-frequency words before they proceed to learning lexical items from specialized word lists (e.g., Coxhead, 2000; Wang et al., 2009). Thus, this section is devoted to briefly introducing two general high-frequency word lists which played important roles in the development of specialized word lists. They are West's (1953) General Service List of English Words (GSL) and Nation's (2012) first 2,000-3,000 BNC/COCA word families. 
To date, West's (1953) GSL is the most well-known and influential general highfrequency word list, which contained the most useful and frequent 2,000 word families of general English. It was developed from a 5-million corpus of written texts based on both objective criteria (frequency and range) and subjective criteria (ease of learning, necessity, cover, style, emotional neutrality). This listing of high-frequency words lays lexical foundation to all kinds of language use in English, including general communication, academic communication and professional communication (Gilner, 2011). The GSL was reported to have a range of $61 \%-85.72 \%$ in coverage across different academic disciplines as summarized in Table 3.2.

Since the GSL was developed more than six decades ago, it does no longer accurately reflect vocabulary in today's English (Coxhead, 2000; Dang \& Webb, 2016; Gardner \& Davies, 2014; Hyland \& Tse, 2007; Richards, 1974). To provide a more accurate picture of the current general high-frequency vocabulary, a number of attempts have been made to build new general high-frequency word lists. The first 2,000-3,000 word families from Nation's (2012) BNC/COCA word lists, Browne's (2014) New General Service List, and Brezina and Gablasova's (2015) New General Service List are cases in point. Among these lists, Nation's (2012) most frequent 2,000 word families have been shown to be the most suitable general high-frequency lists for L2 learners according to criteria such as lexical coverage, learner knowledge, and teacher perception (Dang, 2017). Nation's (2012) most frequent 2,000 BNC/COCA word families are part of an unfinished work of the BNC/COCA word family lists, which have been continuously developed, according to frequency and range data in general English texts, into 28 frequency levels and four supplementary lists (Nation et al., 2016). The first 1,000-word-family list is the most frequently used and widely ranging of all, followed by the second 1,000-word-family list till the less frequent twenty-eighth 1,000-word-family list. Hsu (2013) used the first 3,000 word families of the BNC/COCA as the base in the study to build a medical word list (see Section 3.3.5.2 for details). 
Table 3.2: Coverage of West's (1953) GSL in different specialized corpora

\begin{tabular}{|c|c|c|c|c|}
\hline Study & Discipline & Text type & Size of corpus & Coverage \\
\hline $\begin{array}{l}\text { Sutarsyah, Nation, and } \\
\text { Kennedy (1994) }\end{array}$ & Economics & Textbooks & 300,000 & $82.50 \%$ \\
\hline Ward (1999) & Engineering & Written texts & 60,000 & $82.30 \%$ \\
\hline $\begin{array}{l}\text { Coxhead and Hirsh } \\
\text { (2007) }\end{array}$ & Science & $\begin{array}{l}\text { Textbooks and } \\
\text { other written } \\
\text { materials }\end{array}$ & $1,761,380$ & $71.52 \%$ \\
\hline Fraser (2009) & Pharmacology & $\begin{array}{l}\text { Research } \\
\text { articles }\end{array}$ & 360,000 & $61.00 \%$ \\
\hline Konstantakis (2007) & Business & Textbooks & 600,000 & $85.72 \%$ \\
\hline $\begin{array}{l}\text { Martínez, Beck, and } \\
\text { Panza (2009) }\end{array}$ & Agriculture & $\begin{array}{l}\text { Research } \\
\text { articles }\end{array}$ & 826,416 & $67.53 \%$ \\
\hline $\begin{array}{l}\text { Coxhead, Stevens, and } \\
\text { Tinkle (2010) }\end{array}$ & Science & Textbooks & 279,733 & $76.96 \%$ \\
\hline Li and Qian (2010) & Finance & $\begin{array}{l}\text { Written and } \\
\text { spoken texts }\end{array}$ & $6,279,702$ & $72.63 \%$ \\
\hline Kwary (2011) & Finance & Textbooks & 23,719 & $73.33 \%$ \\
\hline $\begin{array}{l}\text { Khani and Tazik } \\
\text { (2013) }\end{array}$ & $\begin{array}{l}\text { Applied } \\
\text { Linguistics }\end{array}$ & $\begin{array}{l}\text { Research } \\
\text { articles }\end{array}$ & $1,553,450$ & $76.40 \%$ \\
\hline $\begin{array}{l}\text { Valipouri and Nassaji } \\
\text { (2013) }\end{array}$ & Chemistry & $\begin{array}{l}\text { Research } \\
\text { articles }\end{array}$ & 4 million & $65.46 \%$ \\
\hline Liu and Han (2015) & $\begin{array}{l}\text { Environmental } \\
\text { Science }\end{array}$ & $\begin{array}{l}\text { Research } \\
\text { articles }\end{array}$ & 862,242 & $70.16 \%$ \\
\hline $\begin{array}{l}\text { Lu and Durrant } \\
\text { (2017) }\end{array}$ & TCM & $\begin{array}{l}\text { Research } \\
\text { articles }\end{array}$ & $1,045,969$ & $65.72 \%$ \\
\hline
\end{tabular}

Taken together, the high coverage of general high-frequency word lists in different disciplines indicate that general high-frequency words are crucial in ESP vocabulary learning as the vocabulary foundation and that they deserve attention from both ESP learners and teachers. 


\subsubsection{Generic academic word lists}

Generic academic word lists refer to word lists containing important words for academic study across disciplines without being tied to one specific discipline. They have drawn considerable attention from researchers over a long period of time (Browne, Culligan, \& Phillips, n.d.; Campion \& Elley, 1971; Coxhead, 2000; Farrell, 1990; Gardner \& Davies, 2014; Ghadessy, 1979; Lynn, 1973; Praninskas, 1972; Xue \& Nation, 1984). Three influential lists of this kind are Xue and Nation's (1984) University Word List (UWL), Coxhead's (2000) Academic Word List (AWL), and Gardner and Davies' (2014) Academic Vocabulary List (AVL). These word lists lay methodological foundations for the development of discipline-specific word lists, and thus will be introduced briefly in turn in the remaining part of this section.

The UWL was a pioneering academic word list, which was developed by combining four existing academic word lists from Campion and Elley's (1971), Praninskas' (1972), Ghadessy's (1979), and Lynn's (1973) lists. It consisted of 836 word families on top of West's (1953) GSL. Despite its high coverage in academic texts, the UWL was criticized for lacking consistent selection principles in development and having the limitations of its component lists (Coxhead, 2000).

Inspired by the previous work, Coxhead (2000) developed the AWL from a corpus of 3.5 million words of written academic texts based on principled criteria of specialized occurrence, range and frequency. The AWL consists of 570 word families in total, and it remains the most influential academic word lists so far. When tested in specialized corpora of different academic disciplines, the AWL provided coverage from $4.66 \%$ to $12.82 \%$ as shown in Table 3.3 adapted from Coxhead (2011, p. 356).

Though the AWL has great value in EAP pedagogy and research, it has limitations. The major limitation was that the AWL was developed on top of the GSL. The outdated nature of the GSL led to the inclusion of some high-frequency items of the British National Corpus, which is a relatively recent general English corpus (Dang, 2017; Nation, 2004; Schmitt \& Schmitt, 2014). 
Table 3.3: Coverage of Coxhead's (2000) AWL in different academic disciplines

\begin{tabular}{|c|c|c|c|c|}
\hline Studies & Discipline & Text type & Tokens & Coverage \\
\hline $\begin{array}{l}\text { Chen and Ge } \\
\text { (2007) }\end{array}$ & Medicine & Research articles & 190,425 & $10.07 \%$ \\
\hline $\begin{array}{l}\text { Coxhead and Hirsh } \\
\text { (2007) }\end{array}$ & Science & $\begin{array}{l}\text { Textbooks and } \\
\text { other written } \\
\text { materials }\end{array}$ & $1,761,380$ & $8.96 \%$ \\
\hline $\begin{array}{l}\text { Konstantakis } \\
\text { (2007) }\end{array}$ & Business & Textbooks & 600,000 & $4.66 \%$ \\
\hline Fraser (2009) & Pharmacology & Research articles & 360,000 & $9.50 \%$ \\
\hline $\begin{array}{l}\text { Martínez et al. } \\
\text { (2009) }\end{array}$ & Agriculture & Research articles & 826,416 & $9.06 \%$ \\
\hline Ward (2009) & Engineering & Textbooks & 271,000 & $11.30 \%$ \\
\hline $\begin{array}{l}\text { Coxhead et al. } \\
(2010)\end{array}$ & Science & Textbooks & 279,733 & $7.05 \%$ \\
\hline Li and Qian (2010) & Finance & $\begin{array}{l}\text { Written and } \\
\text { spoken texts }\end{array}$ & $6,279,702$ & $10.46 \%$ \\
\hline Kwary (2011) & Finance & Textbooks & 23,719 & $12.00 \%$ \\
\hline $\begin{array}{l}\text { Khani and Tazik } \\
\text { (2013) }\end{array}$ & $\begin{array}{l}\text { Applied } \\
\text { Linguistics }\end{array}$ & Research articles & $1,553,450$ & $11.96 \%$ \\
\hline $\begin{array}{l}\text { Valipouri and } \\
\text { Nassaji (2013) }\end{array}$ & Chemistry & Research articles & 4 million & $9.96 \%$ \\
\hline Liu and Han (2015) & $\begin{array}{l}\text { Environmental } \\
\text { Science }\end{array}$ & Research articles & 862,242 & $12.82 \%$ \\
\hline Yang (2015) & Nursing & Research articles & $1,006,934$ & $8.93 \%$ \\
\hline $\begin{array}{l}\text { Lu and Durrant } \\
\text { (2017) }\end{array}$ & TCM & Journal articles & $1,045,969$ & $10.64 \%$ \\
\hline
\end{tabular}

Being aware of such limitation of existing academic word lists, Gardner and Davies (2014) built the Academic Vocabulary List (AVL) using a different approach. This approach does not assume that learners already have the knowledge of general high-frequency words, and thus regarded the academic words as a separate set of vocabulary cutting across different frequency levels of general English vocabulary. Using lemma as unit of counting, 
Gardner and Davies (2014) selected 3,000 items that had wider range and higher frequency in the 120-millon-word academic corpus than the 305-million-word nonacademic corpus and evenly distributed in the academic corpus in the AVL. The development of the AVL provides us another angle to look at academic vocabulary. However, Durrant (2016) found that even though the AVL was a long listing of academic words, only half of them were truly useful to university student writers because the other items were rarely used in students' academic writing.

Lu and Durrant (2017) examined the usefulness of the AWL and AVL in the dicipline of TCM. They found that the AWL (e.g., significant, analysis) covered $10.64 \%$ as shown in Table 3.3 and the AVL (e.g., group, study) covered $21.17 \%$ of the total tokens. These figures suggest that both generic academic word lists are of great importance in TCM. Nonetheless, such high coverages were achieved by a small number of items in both academic word lists (Lu \& Durrant, 2017). Further analysis of the most frequently used words that were not in the GSL, AWL and AVL showed that a small number of such words (e.g., patient, acupuncture) also achieved a high coverage. These findings suggest that a discipline-specific TCM word list may be of great benefit to the leaners. In the next subsection, research about discipline-specific word lists is reviewed as they are important to the present research.

\subsubsection{Discipline-specific word lists}

Growing research has shown that the generic academic word lists have limited use to learners who have highly specific needs in one discipline (Chen \& Ge, 2007; Durrant, 2016; Hyland \& Tse, 2007; Martínez et al., 2009). Thus, it is suggested that discipline-specific word lists might better serve the needs of such learners. In response to this suggestion, discipline-specific word lists targeted at certain subject areas are established adopting two approaches. Briefly, the first approach builds the lists on top of general highfrequency word lists following the UWL (Xue \& Nation, 1984) and AWL (Coxhead, 2000), while the second approach does not rely on any existing word lists and thus those word lists stand alone. The former approach takes into account learners' existing knowledge of general high-frequency words, but the lists are affected by the limitations of the general word lists on which they are built (Dang, Coxhead, \& Webb, 2017). The latter approach considers the specialized vocabulary as a separate set of vocabulary which cuts across 
different frequency levels in general English (Gardner \& Davies, 2014). Thus, word lists developed using this approach are not affected by the limitations of general word lists. However, it is at risk of repeating lexical items which are already known by the learners (Dang et al., 2017). Table 3.4 and Table 3.5 summarize discipline-specific word lists based on these two approaches respectively.

Table 3.4: Discipline-specific word lists on top of general vocabulary

\begin{tabular}{|l|l|l|}
\hline Author(s) & Word list & Size of the list \\
\hline $\begin{array}{l}\text { Coxhead and Hirsh } \\
(2007)\end{array}$ & Pilot Science Word List & 318 word families \\
\hline Wang et al. (2008) & Medical Academic Word List & 623 word families \\
\hline Hsu (2013) & Medical Word List & 595 word families \\
\hline Hsu (2014a) & Engineering English Word List & 729 word families \\
\hline Liu and Han (2015) & Environmental Academic Word List & 458 word families \\
\hline Yang (2015) & Nursing Academic List & 676 word families \\
\hline
\end{tabular}

Table 3.5: Stand-alone discipline-specific word lists

\begin{tabular}{|l|l|l|}
\hline Author(s) & Word list & Size of the list \\
\hline Ward (1999) & Engineering Word List & 3,000 word families \\
\hline Fraser (2009) & Pharmacology Word List & 2,000 word families \\
\hline Ward (2009) & Basic Engineering English Word List & 299 word types \\
\hline $\begin{array}{l}\text { Valipouri and Nassaji } \\
(2013)\end{array}$ & Chemistry Academic Word List & 1,577 word families \\
\hline $\begin{array}{l}\text { Coxhead et al. (2016) } \\
\text { Lei and Liu (2016) }\end{array}$ & Carpentry List & 1,424 word types \\
\hline $\begin{array}{l}\text { Watson Todd (2017) } \\
\text { Coxhead } \\
\text { Demecheleer (2018) }\end{array}$ & Plumbing word list & 965 lemmas \\
\hline
\end{tabular}

As can been seen in Table 3.4 and Table 3.5, developing discipline-specific word lists has attracted sustained interest especially in the specialized discipline of medicine. In contrast, word lists particularly focused on TCM vocabulary are rare. Thus, it indicates 
that research on the development of word lists in TCM is needed. In the next subsection, studies about medicine-specific word lists will be reviewed in depth.

\subsubsection{Medicine-specific word lists}

This section reviews four studies on medical word lists to closely examine the methods that have been used to develop medical word lists.

Table 3.6: Overview of four word lists in medicine

\begin{tabular}{|c|c|c|c|c|}
\hline Word list & $\begin{array}{l}\text { Medical } \\
\text { Academic Word } \\
\text { List (MAWL) }\end{array}$ & $\begin{array}{l}\text { Medical Word } \\
\text { List (MWL) }\end{array}$ & $\begin{array}{l}\text { Medical Academic } \\
\text { Vocabulary List } \\
\text { (MAVL) }\end{array}$ & $\begin{array}{l}\text { Medical } \\
\text { technical word } \\
\text { lists }\end{array}$ \\
\hline Author(s) & $\begin{array}{l}\text { Wang et al. } \\
\text { (2008) }\end{array}$ & Hsu (2013) & $\begin{array}{l}\text { Lei and Liu } \\
\text { (2016) }\end{array}$ & Quero (2015) \\
\hline $\begin{array}{l}\text { Target } \\
\text { population }\end{array}$ & $\begin{array}{l}\text { Medical English } \\
\text { learners }\end{array}$ & $\begin{array}{l}\text { EFL medical } \\
\text { undergraduates }\end{array}$ & $\begin{array}{l}\text { Medical English } \\
\text { learners }\end{array}$ & $\begin{array}{l}\text { First and second } \\
\text { year medical } \\
\text { students }\end{array}$ \\
\hline Corpus & $\begin{array}{l}\text { 1,093,011 tokens } \\
\text { of medical } \\
\text { journal article }\end{array}$ & $\begin{array}{l}15 \text { million } \\
\text { tokens of } \\
\text { medical } \\
\text { textbooks }\end{array}$ & $\begin{array}{l}2.7 \text { million tokens } \\
\text { of medical journal } \\
\text { articles and } 3.5 \\
\text { million tokens of } \\
\text { medical } \\
\text { textbooks }\end{array}$ & $\begin{array}{l}5,431,740 \\
\text { tokens of } \\
\text { textbooks }\end{array}$ \\
\hline $\begin{array}{l}\text { Main } \\
\text { inclusion } \\
\text { criteria }\end{array}$ & $\begin{array}{l}\text { Specialized } \\
\text { occurrence, } \\
\text { range, frequency }\end{array}$ & $\begin{array}{l}\text { Specialized } \\
\text { occurrence, } \\
\text { range, } \\
\text { frequency }\end{array}$ & $\begin{array}{l}\text { Frequency ratio, } \\
\text { range, dispersion, } \\
\text { discipline } \\
\text { measure, } \\
\text { specialized } \\
\text { meaning }\end{array}$ & $\begin{array}{l}\text { Frequency, } \\
\text { meaning }\end{array}$ \\
\hline $\begin{array}{l}\text { Size of the } \\
\text { list }\end{array}$ & 623 & 595 & 1,234 & 26,000 \\
\hline $\begin{array}{l}\text { Unit of } \\
\text { counting }\end{array}$ & Word family & Word family & Lemma & Word type \\
\hline
\end{tabular}


Table 3.6 above provides an overview of these four medical word lists in comparison the Medical Academic Word List (Wang et al., 2008), the Medical Word List (Hsu, 2013), the New Medical Academic Word List (Lei \& Liu, 2016) and the Medical technical word lists (Quero, 2015). Such review can shed light on the best way to determine items for a TCM-specific word list. Now let us look closely at them in turn.

\subsubsection{Wang et al's (2008) Medical Academic Word List}

Wang et al. (2008) established a medical academic word list (MAWL), which consisted of 623 word families based on a 1.1-million-word corpus of medical journal articles. The development of the MAWL largely followed Coxhead's (2000) approach of establishing the AWL based on selection criteria of specialized occurrence, range, and frequency. That is, the MAWL word families must satisfy the three aforementioned criteria in order to be selected. Specialized occurrence meant the selected word families must be outside West's (1953) GSL. In terms of range, the MAWL word families had to occur in 16 or more of the 32 sub-disciplines. With regard to frequency, the MAWL word families had to occur at least 30 times in the medical corpus (see Table 3.6). The MAWL covered $12.24 \%$ of the running words in the corpus on which it was developed. Such high coverage highlighted the idea that a discipline-specific lexical repertoire may better serve the needs of ESP learners.

The MAWL had three limitations. The first limitation was that the MAWL was developed on top of the GSL, which means the MAWL was affected by the limitations of the GSL. For example, one common criticism of the GSL is that the age of the GSL has rendered it inaccurate in reflecting vocabulary in today's English (Coxhead, 2000; Gardner \& Davies, 2014; Hyland \& Tse, 2007; Richards, 1974). Second, the authors of the MAWL claimed that this list targets at academic vocabulary, which is a class of words between technical and non-technical words (Wang et al., 2008). Nonetheless, the criterion for determining which words are technical and which words are academic remains unclear. For example, words such as lesion and vein tend to be considered as technical vocabulary in medicine but they were still included because they are frequently used across different medical areas. Third, the MAWL was developed from a single genre of journal articles which represented the writing of experts in this field, and thus it might be of limited use to the beginners of this field such as undergraduate students. Despite these limitations, the MAWL was still a pioneering discipline-specific word lists which inspired further 
research of discipline-specific word lists (e.g., the Nursing Academic Word List of Yang, 2015).

\subsubsection{Hsu's (2013) Medical Word List}

Targeting mainly at undergraduates, Hsu (2013) developed a medical word list (MWL) based on a medical textbook corpus of 15 million tokens. The selection criteria of the MWL also followed the AWL (Coxhead, 2000), which were range, specialized occurrence, and frequency. Nonetheless, the specialized occurrence and frequency criteria were adopted with adjustments. Different from the AWL, specialized occurrence meant the selected items had to be outside the most frequent 3,000 word families of Nation's (2012) BNC/COCA lists because the students in her context were supposed to have an English vocabulary of at least the most frequent 3,000 word families. In addition, the frequency of at least 863 times in this medical textbook corpus was set out of practical reasons so that the MWL was not too cumbersome. Further, an MWL word family had to occur in more than half of the sub-disciplines to be selected. The selection criteria led to a listing of 595 word families, which were mainly sub-technical (e.g., component, syndrome) and lay-technical words (e.g., immune, enzyme) in medicine. Despite the fact that lay-technical vocabulary was included in the MWL, highly technical words (with Greek/Latin components, e.g., arthrocentesis, olecranarthropathy) were not included because Hsu (2013) believed that such highly technical vocabulary has constancy in denotation and is thus easy to learn. Another reason for not focusing on highly technical words was that they would be taught in courses such as Medical Terminology (Hsu, 2013).

The MWL is a focused medicine-specific word list, which contributed $10.72 \%$ to the coverage in the medical textbook corpus. It shed light on the nature of vocabulary between non-technical and highly technical vocabulary. Specifically, those words were classified into six groups: (1) words expressing academic notions (e.g., component, mechanism), (2) words whose technical meaning emerged from the medical context (e.g., susceptible to colds, skin graft), (3) words with equal general and specialized meaning (e.g., protein, vein), (4) words originally used in medical contexts but may undergo a semantic change when used in general language (e.g., 'morbid anatomy' versus 'morbid interest in horror movies'), (5) words mainly used in medical register but can be easily understood by layperson (e.g., gene, hormone), (6) words used exclusively in medical contexts (e.g., hyperplasia, thrombosis). 
The MWL is not without limitations. First, the MWL excluded the most frequent 3,000 BNC/COCA word families with the assumption that the EFL learners in that context had knowledge of these words. However, there is no empirical evidence showing whether these learners understand all members of the most frequent 3,000 BNC/COCA word families when these take on a technical meaning in medicine, as in the case of words in group (2) mentioned above. Second, fully technical vocabulary, which was not the concern of the MWL, actually meant the 1,380 items from one medical terminology book in this study. Such a short listing of medical words was not enough to represent all the fully technical words in medicine. This actually led to inclusion of such fully technical words (e.g., thrombosis) in the MWL. As a result, the MWL might not fully match the aim of building a word list to bridge the gap of non-technical and technical vocabulary in medicine.

\subsubsection{Lei and Liu's (2016) Medical Academic Vocabulary List}

Based on the view that academic vocabulary is a separate set of vocabulary cutting across different frequency levels of general vocabulary, Lei and Liu (2016) developed a lemmabased medical academic vocabulary list (MAVL) based on a 2.7-million-word journal article corpus and a 3.5-million-word textbook corpus. To be selected in the MAVL, a lexical item has to satisfy six criteria which combine Coxhead (2000) and Gardner and Davies' (2013) criteria for making the AWL and AVL respectively. The first criterion is a minimum frequency of 28.57 times per million words. The second criterion is a frequency ratio of 1.5 , which meant a word has to occur $50 \%$ more often in the medical corpora than the same word in a general English corpus. In terms of the third criterion, a lexical item has to occur with at least $20 \%$ of the expected frequency in 12 out of 21 sub-corpora in this study. To ensure a lemma is distributed evenly over the corpora, a Juilland's D (Juilland, Brodin, \& Davidovitch, 1970) value of 0.5 is adopted as the fourth criterion. In addition, a lemma has to occur in more than 3 out of 21 sub-corpora to satisfy the discipline measure. Lastly, a lemma has to satisfy a special meaning criterion if this lemma appeared in the New General Service List of Brezina and Gablasova (2015). The meaning criterion is that this lemma can be found in two of the selected medical dictionaries, which shows that this lemma is a medical word. 
All MAVL lemmas were first selected in the medical journal article corpus, and then validated in the medical textbook corpus mentioned above. A total of 965 lemmas which satisfied the selection criteria in both medical corpora appeared in the MAVL. They covered $19.44 \%$ and $20.18 \%$ of the medical journal article corpus and textbook corpus respectively, whereas the lemmatized MAWL covered $10.52 \%$ and $12.97 \%$ respectively. A possible reason for this discrepancy in the Medical Academic Vocabulary List and Medical Academic Word List might be that the former did not exclude the high-frequency words which have a medical meaning. It implied that general high-frequency words which have technical meanings can be very important and useful to the learners, and thus they should not be excluded from the discipline-specific word lists. Given the high coverage of such a relatively small set of vocabulary, this study substantiated the idea that a restricted, discipline-specific lexical repertoire may better serve learners' needs. However, similar to Wang et al.'s (2008) Medical Academic Word List, this list did not include narrowly-distributed technical vocabulary in medicine such as arrhythmia and genomic (Lei \& Liu, 2016). The reason to exclude such technical words is that these words are too specialized. However, Lei and Liu (2016) pointed out that those technical words are useful for the study of specialist areas, and thus should be the focus of future research. As a result, this research indicates that future research on discipline-specific word list construction should take such narrowly-distributed technical vocabulary into account.

\subsubsection{Quero's (2015) Medical technical word lists}

Quero (2015) developed 26 medical word lists along the investigation of vocabulary load of medical textbooks. Each of the 26 medical word list consisted of 1,000 word types selected from a 5.4-million running word corpus based on the criteria of frequency ratio (see Section 2.3.1 for calculation of frequency ration) and meaning. To be selected, a word type had to occur more frequently in the medical corpus than the general corpus or be unique to the medical corpus. At the same time, this word had to have a technical meaning according to the semantic rating scale (see Section 2.3.2 for details). Since the main aim of Quero's (2015) study was to examine the vocabulary load of technical textbooks, she included almost all technical word types. This led to the development of three medical word lists having higher frequency in the medical corpus than the general corpus, and 23 word lists unique to the medical corpus. 
These medical word lists provide valuable information on the technical vocabulary in medicine. Such medical word lists, however, have two main limitations. First, 26 medical word lists with 1,000 word types in each list are very long. Second, they include more than 2,000 medical word types with occurrence of one time in the over 5-million corpus. Therefore, it cannot be readily used by learners and teachers.

\subsubsection{Summary of medicine-specific word lists}

Section 3.3.5 has reviewed research on medicine-specific word lists for medical learners who have highly specific lexical needs with a focus on medicine-specific word lists. These lists indicated that the discipline-specific word lists can well serve ESP learners' needs. However, even though there are a number of medicine-related word lists available, only one (Quero, 2015) of them included technical vocabulary. For the one which mainly concerned technical vocabulary in medicine, the main aim was not to provide a pedagogical word list but to investigate the vocabulary burden of medical textbooks. This is mainly because most researchers believed that technical words were not a problem for the ESP learners (Hsu, 2013; Lei \& Liu, 2016; Wang et al., 2008). However, research has shown that insufficient knowledge about technical vocabulary is one of the most challenging aspects in ESP learning (Ardasheva \& Tretter, 2015; Evans \& Morrison, 2011; Gablasova, 2015; Wu \& Hammond, 2011). Therefore, research about the development of discipline-specific word lists that include technical vocabulary is still needed in medicinerelated disciplines. Such research can provide us insight into the nature of technical vocabulary. Moreover, even though TCM is a medicine-related discipline, it differs from general Western medicine since TCM and Western medicine represent two different ways of thinking and two different ways of seeing the world (Kaptchuk, 2001). Developing a TCM-specific word list can thus be even more valuable as there is only one word list study about TCM in the literature, which is partially reported in a conference proceeding (Hsu, 2016). Such research should take the issues of previous word list studies into account. Further, the assumption that the ESP learners do not have problem in technical vocabulary should also be tested in empirical research.

\subsection{Making use of the existing word lists: Vocabulary profile analysis}

As mentioned in the previous sections, the general high-frequency word lists and generic academic word lists are of great importance since those lists are the basis for vocabulary learning, teaching and researching. Vocabulary profile analysis is a good example of 
making use of such well-constructed general word lists for research. A number of researchers have utilized the existing word lists to conduct lexical profile analysis to understand the lexical characteristics of a certain discipline or register (e.g., Dang \& Webb, 2014; Hsu, 2013; Hsu, 2014a). A good understanding of the lexical characteristics can help the researchers make informed decision about further investigation of the technical vocabulary. This section examines research on lexical profile analysis to show how to make use of the existing word lists to understand the lexis or lexical demands of a certain genre or discipline.

Hsu (2014a) examined the vocabulary demands of English-medium engineering textbooks via lexical profile analysis. Nation's (2006) 14 BNC/COCA word lists served as base word lists in the analysis. Specifically, the coverage of each BNC/COCA word list over the target corpus was calculated and then the vocabulary load, in the number of base word lists, needed to reach 95\% lexical coverage was counted. The rationale for this investigation rested on two ideas. First, Hsu's (2014) study built on the premise that there is a linear relationship between lexical coverage and reading comprehension (Schmitt, Jiang, \& Grabe, 2011) and 95\% coverage is often considered as the minimal reading comprehension threshold (Laufer \& Ravenhorst-Kalovski, 2010). Second, this study rested on the idea that the learners, both native and non-native speakers, acquire vocabulary largely in the order of its frequency and range (Nation, 2006). In other words, the higher-frequency and wider-range words are more likely to be encountered and thus acquired before the lower-frequency and narrower-range words. As a result, Hsu (2014a) found that the vocabulary load (to reach 95\% coverage) of the engineering textbooks is 5,000 word families plus proper nouns, transparent compounds and abbreviations (see Table 3.7).

Using a similar approach, a number of other researchers have investigated the lexical demands of various genres as summarized in Table 3.7. These studies used 95\% lexical coverage as the minimally acceptable comprehension threshold (Laufer \& RavenhorstKalovski, 2010) and/or 98\% lexical coverage as the optimal comprehension threshold (Hu \& Nation, 2000; Schmitt, Jiang, et al., 2011). Although lexical coverage is believed to be the most influential factor, it should be noted that other factors (e.g., syntactic structure) may also affect comprehension (Laufer \& Sim, 1985), and these were not 
considered in those studies. These studies showed that different text genres have varying vocabulary demands. Among them, it is not surprising to notice that life and medical science spoken texts have a heavier vocabulary burden compared to parallel spoken texts in other disciplines (Dang \& Webb, 2014). This finding indicates that life and medical science generally have a heavy vocabulary load.

Table 3.7: Summary of vocabulary load studies

\begin{tabular}{|c|c|c|c|}
\hline Study & Text type & $\begin{array}{l}\text { Vocabulary load } \\
\text { at } 95 \% \text { coverage }\end{array}$ & $\begin{array}{l}\text { Vocabulary load } \\
\text { at } 98 \% \text { coverage }\end{array}$ \\
\hline \multirow{2}{*}{$\begin{array}{l}\text { Nation } \\
(2006)\end{array}$} & Novels & 4,000 & $8,000-9,000$ \\
\hline & Newspapers & 4,000 & $8,000-9,000$ \\
\hline $\begin{array}{l}\text { Webb and } \\
\text { Rodgers } \\
(2009 a)\end{array}$ & Movies & 3,000 & 6,000 \\
\hline $\begin{array}{l}\text { Webb and } \\
\text { Rodgers } \\
(2009 b)\end{array}$ & TV programs & $2,000-4,000$ & $5,000-9,000$ \\
\hline \multirow[t]{2}{*}{ Hsu (2014a) } & Business textbooks & 3,500 & 5,000 \\
\hline & Business research articles & 5,000 & 8,000 \\
\hline \multirow{5}{*}{$\begin{array}{l}\text { Dang and } \\
\text { Webb } \\
(2014)\end{array}$} & Academic spoken English texts & 4,000 & 8,000 \\
\hline & $\begin{array}{l}\text { Arts and Humanities spoken } \\
\text { texts }\end{array}$ & 4,000 & 7,000 \\
\hline & $\begin{array}{l}\text { Life and Medical Science } \\
\text { spoken texts }\end{array}$ & 5,000 & 13,000 \\
\hline & Physical Sciences spoken texts & 4,000 & 10,000 \\
\hline & Social Sciences spoken texts & 3,000 & 5,000 \\
\hline Hsu (2014a) & Engineering textbooks & 5,000 & NA \\
\hline
\end{tabular}

(Note: The unit of counting in the above studies are word families; NA = Not available.)

It is also through such vocabulary profile analysis that Hsu (2013) found the first 3,000 high-frequency word families plus the medical terminology and abbreviations only accounted for $87.28 \%$ of the running words in the medical textbooks in her study. This coverage was $10.72 \%$ short of the $98 \%$ lexical coverage. Such results indicate that there 
is a huge gap between the first 3,000 high-frequency words and the technical vocabulary in medical textbooks. Thus, Hsu (2013) created the Medical Word List (see Section 3.3.5.2 for details) to bridge this gap.

To sum up, these studies inform us that well-constructed word lists can be used to estimate the vocabulary level of the texts being studied and help understand their lexical characteristics by analyzing what proportion of the vocabulary is at the low-frequency levels or even outside them. Such analysis could also lay a foundation for further investigation (e.g., developing a pedagogical word list).

\subsection{Summary}

This chapter has provided an overview of the place of technical vocabulary in vocabulary learning. It shows that vocabulary is a macro construct which can be classified into different micro constructs - high, mid and low-frequency vocabulary, or general highfrequency, academic, technical and low-frequency vocabulary. Among the types of vocabulary, technical vocabulary is the main focus given that this research is located in the context of Traditional Chinese medicine. Then, it looked at the development and use of word lists as a way to organizing the large amount of vocabulary for learning and teaching including technical vocabulary. The final section reviewed important studies that made use of well-constructed word lists to understand the lexical characteristics in the targeted discourse community.

Based on the review of literature in the current chapter, two more research gaps have been identified. First, previous studies have shown that developing frequency-based word lists is an effective way to organize the large amount of vocabulary for learning purposes. Further, research also indicated that discipline-specific word lists can better serve ESP learners' needs than generic academic word lists. Thus, it will be beneficial to develop a TCM word list, especially a TCM word list which includes technical words. This kind of list can not only be of pedagogical use to the learners and teachers, but can also shed light on the nature of technical vocabulary in this far underexplored area.

Second, there is little dispute that a frequency-based word list is an important pedagogical tool. With a view to the usefulness of such frequency-based word lists, 
Martinez and Schmitt (2012) suggested that the potential difficulty of the lexical items for the learners should also be taken into consideration. Nonetheless, it has been indicated in Section 2.6 that little is known about the actual difficulty that the ESP learners encounter. Therefore, it is useful to investigate learners' understanding of the technical words so as to obtain a better idea of the difficulty they encounter. Further, given the cultural differences between Chinese and Western conceptions of body and health, further research on technical vocabulary in TCM should consider the variation in learners' cultural background to better support their technical vocabulary development.

The remainder of this thesis will bridge the gaps that remain outstanding from the literature review (Chapter 2 and 3). The first study (Chapter 4) investigates lexical characteristics and estimates lexical burdens of vocabulary in the underexplored discipline of TCM to gain a comprehensive understanding of TCM vocabulary overall. Study 2 (Chapter 5) investigates technical vocabulary including single and multiword units, while Study 3 (Chapter 6) explores TCM learners' understanding of technical words. In the following chapter, the first study which examines lexical characteristics and vocabulary load in TCM will be presented because this study functions as the basis of Study 2 and 3. 


\section{Chapter 4 Lexical profile and vocabulary loads of TCM texts}

\subsection{Introduction}

The main purpose of this chapter is to explore the vocabulary of English-medium TCM textbooks and journal articles to have a comprehensive understanding of the nature of vocabulary in the specialized discipline of TCM so as to provide TCM learners with support in specialized vocabulary learning. To achieve this purpose, the lexical profile of TCM vocabulary was analyzed independently and also in comparison with that in Western medicine and general written English through corpus analysis. This study (henceforth Study 1) addresses the following the research questions (RQs):

1. What kind(s) of vocabulary make up the English-medium TCM texts?

1a. What is the lexical profile of TCM vocabulary along Nation's (2012) BNC/COCA base word lists?

1b. Beyond Nation's (2012) BNC/COCA base word lists, what vocabulary remains given its cultural and linguistic roots and how can it be classified?

2. What is the coverage of Schmitt and Schmitt's (2014) high, mid, and low-frequency vocabulary in the TCM Corpora?

3. What is the vocabulary load of the English-medium TCM texts?

4. What is the vocabulary load of each type of TCM texts in the TCM Corpora?

5. To what extent does TCM vocabulary differ from that of the Western Medicine?

6. To what extent does TCM vocabulary differ from that of General Written English?

Chapter 4 begins with the description of the methodology of the present study, including the process of corpus building, instrument for data analysis, and process of data analysis in the present study. Specifically, Section 4.2 and 4.3 describe the process of data collection, while Section 4.4 focuses on data analysis of the corpus data. Then, Section 4.5 presents the results of the analysis. Section 4.6 describes the main limitations of the present study. Section 4.7 ends this chapter by summarizing the main findings of the present study and presenting the rationale for the next study. 


\subsection{Building the TCM Corpora}

In corpus-based studies, one key issue is how to build a representative corpus which can suitably represent the language use and reliably reflect the linguistic variation in the target discourse domain. Therefore, this section will start with a review of how to build a representative corpus. The remaining of this section will describe the processes of how the Traditional Chinese Medicine textbook and journal article corpora were built.

\subsubsection{How to build a representative corpus?}

As proposed by Biber (1993), three principles should be followed when designing a representative corpus: (1) defining the population that the target corpus intends to represent; (2) choosing a sampling frame to guide the sampling of the target population, and (3) evaluating the extent to which the selected sample represents the target population. Of these principles, defining the target population is a prerequisite to making sampling decisions and evaluating the sampling decisions.

Guided by Biber's (1993) principles of designing representative corpus, the target population was first defined in order to study the vocabulary in TCM. They are: the TCM textbooks (in the TCM textbook corpus) and the TCM journal articles (in the TCM journal article corpus) used widely by TCM undergraduates. Textbooks and journal articles are two most prominent genres that TCM students are mostly likely to read in their course of study. Textbooks are the main materials that the TCM students encounter especially at the beginning of study while journal articles act as supplementary materials. However, the further they go in their study, the more likely they need to read journal articles. Gradually, when they become professionals, they rely mainly on journal articles. The same pattern has been observed by Lei and Liu (2016) in general medicine. Lei and Liu (2016) used a journal article corpus as primary corpus of establishing a medical academic word list since they targeted both medical students and professionals. Considering that the main target user groups in the present study are students who are studying a Bachelor's degree in TCM, the main materials used in this present research are textbooks with journal articles being the supplementary materials. Having decided on the target population of the TCM Corpora, the next subsections describe how the TCM Corpora were built taking the second and third principles into consideration. In the concluding subsection, a summary of the corpora built in this study is provided. 


\subsubsection{Building the TCM textbook corpus}

Based on my observation as a previous TCM student, TCM learners usually start with the TCM theories and then proceed to clinical practices. A similar course structure was found by Wette and Hawken (2016) for medical students at University of Auckland. Textbooks used at these two stages are different. Based on this consideration, the TCM textbook corpus was sampled equally in theory-based textbooks and practice-based textbooks. Each type of textbooks was built into a sub-corpus: the theory-orientated textbook subcorpus consists of textbooks used in the pre-clinical theory-based years, and the practiceorientated textbook sub-corpus consists of textbooks used in courses about clinical practices. This is a novel but pedagogically friendly way of building the textbook corpus, which can appropriately represent language use in various textbooks of TCM.

In order to select textbooks to build a representative textbook corpus, institutions at the tertiary level which provide TCM Bachelor's degrees were identified via the Internet. Specifically, ten institutions were researched across China (including Hong Kong and Macau), New Zealand, the U.S., the U.K. and Australia which offer English-medium instruction on TCM. The most widely used TCM textbooks were identified. A lecturer from New Zealand School of Acupuncture and Traditional Chinese Medicine was then consulted about the textbooks they used to compare with the lists of textbooks found through the Internet search. As a result, I found that the English-medium TCM textbooks used in institutions from different countries overlap to a considerable extent. Six theorybased textbooks and five practice-based textbooks, which were used in multiple contexts, were selected. Table 4.1 and Table 4.2 demonstrates the composition of the theory-based textbook sub-corpus and practice-based textbook sub-corpus respectively. As shown in these two tables, the size of the textbooks varies from 88,043 tokens to 450,132 tokens. All of the textbooks selected in this study were published after 2000, which means they can represent recent language use in TCM textbooks. 
Table 4.1: Overview of TCM theory-based textbook sub-corpus

\begin{tabular}{|c|c|c|c|c|c|c|}
\hline \multirow{7}{*}{$\begin{array}{l}\text { Theory- } \\
\text { based } \\
\text { textbooks }\end{array}$} & Title & Author(s) & Year & Tokens & Locations & $\begin{array}{l}\text { Total } \\
\text { Tokens }\end{array}$ \\
\hline & $\begin{array}{l}\text { A comprehensive } \\
\text { guide to Chinese } \\
\text { medicine }\end{array}$ & $\begin{array}{l}\text { Ping Chung } \\
\text { Leung, Charlie } \\
\text { Changli Xue, \& } \\
\text { Yung-Chi Cheng }\end{array}$ & 2003 & 92,189 & $\begin{array}{l}\text { China, the } \\
\text { U.S. }\end{array}$ & \multirow{6}{*}{$\begin{array}{l}1,171,6 \\
25\end{array}$} \\
\hline & $\begin{array}{l}\text { A manual of } \\
\text { acupuncture }\end{array}$ & $\begin{array}{l}\text { Peter Deadman, } \\
\& \quad \text { Mazin Al- } \\
\text { Khafaji }\end{array}$ & 2007 & 256,067 & \multirow{5}{*}{$\begin{array}{l}\text { Australia, } \\
\text { China, } \\
\text { New } \\
\text { Zealand, } \\
\text { the U.K., } \\
\text { the U.S. }\end{array}$} & \\
\hline & $\begin{array}{ll}\text { Chinese } & \text { herbal } \\
\text { medicine } & \end{array}$ & $\begin{array}{l}\text { Chongyun Liu, } \\
\& \\
\text { Angela Tseng }\end{array}$ & 2005 & 156,866 & & \\
\hline & $\begin{array}{l}\text { Running a safe } \\
\text { and successful } \\
\text { acupuncture }\end{array}$ & Zhenzhu, Hong & 2006 & 88,043 & & \\
\hline & $\begin{array}{l}\text { The foundations } \\
\text { of } \\
\text { medicine }\end{array}$ & $\begin{array}{l}\text { Maciocia, } \\
\text { Giovanni }\end{array}$ & 2005 & 487,961 & & \\
\hline & $\begin{array}{l}\text { The Web that has } \\
\text { no Weaver }\end{array}$ & Ted, Kaptchuk & 2001 & 90,499 & & \\
\hline
\end{tabular}

Table 4.2: Overview of TCM practice-based textbook sub-corpus

\begin{tabular}{|c|c|c|c|c|c|c|}
\hline \multirow{6}{*}{$\begin{array}{l}\text { Practice- } \\
\text { based } \\
\text { textbooks }\end{array}$} & Title & Authors & Year & Tokens & Locations & $\begin{array}{l}\text { Total } \\
\text { Tokens }\end{array}$ \\
\hline & $\begin{array}{l}\text { Fluid physiology } \\
\text { and pathology in } \\
\text { traditional } \\
\text { Chinese medicine }\end{array}$ & Steven Clavey & 2003 & 198,416 & $\begin{array}{l}\text { Australia, } \\
\text { China, the } \\
\text { U.S., }\end{array}$ & \multirow{5}{*}{$\begin{array}{l}1,109,7 \\
01\end{array}$} \\
\hline & $\begin{array}{l}\text { The treatment of } \\
\text { diabetes with } \\
\text { Chinese medicine }\end{array}$ & $\begin{array}{l}\text { Bob Flaws, } \\
\text { Lynn Kuchinski, } \\
\& \quad \text { Robert } \\
\text { Casanas }\end{array}$ & 2002 & 225,685 & \multirow{2}{*}{$\begin{array}{l}\text { Australia, } \\
\text { China, } \\
\text { New } \\
\text { Zealand }\end{array}$} & \\
\hline & $\begin{array}{l}\text { Treatment of } \\
\text { Infertility with } \\
\text { Chinese medicine }\end{array}$ & Jane Lyttleton & 2013 & 128,318 & & \\
\hline & $\begin{array}{l}\text { The practice of } \\
\text { Chinese medicine }\end{array}$ & $\begin{array}{l}\text { Giovanni, } \\
\text { Maciocia }\end{array}$ & 2008 & 450,132 & $\begin{array}{l}\text { Australia, } \\
\text { China, } \\
\text { New } \\
\text { Zealand, } \\
\text { the U.K., } \\
\text { the U.S. }\end{array}$ & \\
\hline & $\begin{array}{l}\text { Pediatric } \\
\text { acupuncture }\end{array}$ & May, Loo & 2005 & 107,150 & $\begin{array}{l}\text { Australia, } \\
\text { China }\end{array}$ & \\
\hline
\end{tabular}


All aforementioned textbooks were purchased at online shops as e-textbooks in PDF format. The PDF files were converted into TEXT files manually via copying and pasting. Tables, figures, pictures, formulas, Chinese characters, bibliography, and footnotes were manually removed. Acknowledgements and table of contents were also excluded. Errors generated from the conversion of PDF format to TEXT format were checked and corrected manually. Removing such errors in corpus studies is necessary as highlighted by Nation (2016b). After those steps, the theory-orientated textbook sub-corpus and the practice-orientated textbook sub-corpus were built, containing 1,171,625 tokens and 1,109,701 tokens respectively as shown in Table 4.1 and Table 4.2.

Together, the textbook corpus consists of 2,281,326 tokens. By combining internet search, my observation as a TCM students and expert consultation, I believe that the TCM textbook corpus is a balanced and representative corpus, which allows for accurate reflection of language variation in textbooks of the target discourse community.

\subsubsection{Building the TCM journal article corpus}

The journal article corpus was originally built to conduct a vocabulary analysis of academic vocabulary in TCM as one of my Master's assignments, which consisted of only around 1,000,000 words. Later, it was expanded for two purposes. The first purpose is to publish the assignment as an article, which was actually published in March 2017 (Lu \& Durrant, 2017). The second purpose is to conduct the present research.

Unlike textbooks, it can be difficult to capture what kind of the journal articles the learners might encounter at different stages of studying. To select articles which can represent as closely as possible the genre of journal articles that the target users are likely to encounter in their study, the first step was to consult the reference lists obtained through internet search and expert consultation as mentioned above. Four scholarly journals were thus selected, namely Chinese Medicine, Journal of Traditional Chinese Medicine, BMC Complementary and Alternative Medicine, and Complementary Therapies in Medicine. All the journals were international quarterlies and were open to the public. Chinese Medicine and Journal of Traditional Chinese Medicine reported clinical and theoretical TCM research only, while BMC Complementary and Alternative Medicine and Complementary Therapies in Medicine included all sorts of clinical and theoretical 
research about complementary medicine. In the latter case, only articles related to TCM were considered and selected. The second step was to skim the titles of articles in the latest issues. It was found that the TCM research can be roughly divided into three categories, namely: Chinese herbal medicine, including component analysis pharmacology and toxicology; alternative therapies, including acupuncture, moxibustion, auricular acupuncture and scraping therapy; and basic research into theories, diagnoses, and other general aspects of TCM. Therefore, the TCM journal article corpus was sampled in these three categories. The third step was to choose random sample articles of each category with similar size.

These articles were downloaded as PDF files from those four journals. The PDF files were then converted into TEXT files manually via copying and pasting. Any errors generated from the conversion of PDF files to TEXT files were manually checked and corrected. All articles included in the corpus were published between the years 2008 and 2016. Charts, diagrams, images, and Chinese characters written in Chinese were manually removed from the articles. Bibliographies, index numbers, acknowledgements, and appendices were also excluded. The final journal article corpus contained 342 articles, totaling $1,196,941$ tokens as listed in Table 4.3.

Table 4.3: Overview of TCM journal article corpus by journals

\begin{tabular}{|l|l|l|}
\hline Journal & Number of articles & Tokens \\
\hline Chinese herbal medicine & 160 & 399,103 \\
\hline Alternative therapies & 162 & 402,384 \\
\hline $\begin{array}{l}\text { Other general research about TCM theories and } \\
\text { diagnoses }\end{array}$ & 158 & 395,454 \\
\hline Total & 342 & $1,196,941$ \\
\hline
\end{tabular}

Table 4.4: Summary of the TCM Corpora

\begin{tabular}{lll}
\hline Corpus & Sub-corpus & Size \\
\hline \multirow{2}{*}{ TCM textbook corpus } & Theory-based textbook sub-corpus & $1,171,625$ \\
\cline { 2 - 3 } & Practice-based textbook sub-corpus & $1,109,701$ \\
\hline $\begin{array}{l}\text { TCM journal article } \\
\text { corpus }\end{array}$ & None & $1,196,941$ \\
\hline Total & & $3,478,267$ \\
\hline
\end{tabular}


Table 4.4 summarizes the TCM Corpora developed in this study to investigate the lexis in TCM. So far, the development of the TCM Corpora has been completed. In the next section, the development of Western Medical Corpora will be described to make comparison of lexis with TCM.

\subsection{Developing the Western Medical Corpora}

To compare the lexis in TCM and that in Western Medicine (WM), Western Medical Corpora (WM Corpora) was developed. Table 4.5 provides an overview of the WM Corpora. As shown in Table 4.5, the WM Corpora have a similar structure as the TCM Corpora, consisting of a WM textbook corpus and a WM journal article corpus in order to allow fair comparison. Both WM textbook and journal article corpora have similar sizes to the corresponding corpus in TCM.

Table 4.5: Overview of Western Medical Corpora

\begin{tabular}{lll}
\hline Corpus & Source & Size \\
\hline WM textbook corpus & Cecil textbook of internal medicine & $1,134,755$ \\
& Harrison's Principles of Internal Medicine & 809,548 \\
& Merck Manual of Diagnosis and Therapy & $1,115,029$ \\
\hline WM journal article corpus & Medical section of COCA & $1,098,151$ \\
\hline Total & & $4,157,483$ \\
\hline
\end{tabular}

As shown Table 4.5, the WM textbook corpus contains 3,059,332 running words. It includes texts from three textbooks as summarized in Table 4.5: Cecil textbook of internal medicine (Goldman \& Ausiello, 2008), Harrison's Principles of Internal Medicine (Fauci et al., 2008), and Merck Manual of Diagnosis and Therapy (Porter \& Kaplan, 2011). All the materials were obtained from my colleague, Dr. Betsy Quero, who conducted extensive research about vocabulary in Western medicine at Victoria University of Wellington. Her original medical corpus consists of more than five medical textbooks which are widely used by medicine students and professionals. The medical textbook corpus was cut down to the similar size as the TCM textbook corpus. It is advantageous to have Dr. Quero's medical corpus because her medical corpus can be readily analyzed by the Range program (Heatley, Nation, \& Coxhead, 2002). 
The WM journal article corpus contained 1,099,327 running words (see Table 4.5). It was downloaded from the medicine section of the Corpus of Contemporary American English (COCA) (Davies, 2008-). In total, there were 532 journal articles from various medical journals in the WM journal article corpus.

\subsection{Data analysis}

In the previous sections, the development of the TCM and WM Corpora has been described. This section presents how the corpus data were analyzed. It starts with a detailed introduction of the instrument used for data analysis, followed by processing of corpus data, adapting the BNC/COCA base word lists, and categorizing the remaining 'Not in the list' word types. This section ends with the procedure of the analysis.

\subsubsection{The instrument for analysis}

The Range program (Heatley et al., 2002) was used to analyze the corpora data (http://www.vuw.ac.nz/lals/staff/paul-nation/nation_aspx). It enables users to analyze the lexical coverage provided by certain base word lists in a single text or in a large collection of texts. It can also provide frequency and coverage of each word involved in the base word lists. Nation's (2012) BNC/COCA base word lists were adopted as base word lists in order to find out what vocabulary makes up the TCM texts, which consist of word families from the most frequent 1,000 to 25,000 word levels (BASEWRD 1 to 25) and four additional lists (the proper noun, marginal word, transparent compound, and abbreviation lists) (see Table 4.6 for details).

\section{Table 4.6: Nation's (2012) BNC/COCA base word lists}

\begin{tabular}{|l|l|l|}
\hline Basewrd & Components & Examples \\
\hline Basewrd 1-25 & $\begin{array}{l}\text { Word families based on frequency and } \\
\text { range }\end{array}$ & visit, labour, asperity \\
\hline Basewrd 31 & Proper nouns & England, John, Clifton \\
\hline Basewrd 32 & Marginal words & phew, ah, B \\
\hline Basewrd 33 & Transparent compounds & eyeball, halfway, shortsighted \\
\hline Basewrd 34 & Abbreviations & ATM, HP, SVQ \\
\hline
\end{tabular}

To put it simply, the BNC/COCA base word lists were the scale of measurement to find out what kind(s) of vocabulary make up the TCM texts. The reason for using Nation's 
(2012) BNC/COCA base word lists is because they were systematically and continuously developed from the most the high-frequency 1,000 word families till the very lowfrequency 25,000 word families according to how frequently they occur in general English. It thus allows for estimation of vocabulary at various frequency level in complicated texts like the TCM texts. In the BNC/COCA word lists, the word unit of counting is word family. Therefore, the word unit of analysis in this study is also word family to conform to the BNC/COCA base word lists. The criteria used to make word families by Nation (2012) are Bauer and Nation's (1993) Level 6 of making word families, which has been discussed in literature review of Section 3.3.1.

The TCM Corpora were run over the Range program. It was found that $10.19 \%$ of the tokens were not covered by any of the lists and were thus put in the category of 'Not in the lists'. The 'Not in the lists' words were then examined. This led to three additional steps in data analysis: processing the TCM Corpora, adapting the existing BNC/COCA base word lists and categorizing the remaining word types. To ensure that the off-list word types have been grouped into the appropriate lists, each type was crossed-checked by searching the corpora and the meaning were looked up in dictionaries (e.g., the MerriamWebster Dictionary) before being put into the corresponding lists. In the next three subsections, they will be described in detail with justifications provided.

\subsubsection{Processing the TCM Corpora}

There was a considerable number of hyphenated words (e.g., spleen-qi, long-term, reestablish, Dong-yuan), which were listed in the category of 'not in the lists' by Range. Usually, the hyphens are used to indicate a close grammatical or semantic relationship between the hyphenated items (Nation et al., 2016). The items before or after the hyphens can be an affix or a word that can stand alone by itself and has been included in the base word lists (Nation et al., 2016). However, they were counted as new lexical items by the Range program, which had negative effects on analyzing vocabulary because counting such items as new lexical items led to repetitive calculation. To obtain more meaningful results, it was decided to process different uses of hyphens in two different ways with the principle of being consistent with the BNC/COCA base word lists. 
In most cases, the hyphens of hyphenated compounds (e.g., spleen-qi, five-element) were replaced by spaces so that they could be reclassified by corresponding separate single words (e.g., spleen qi, five element). In other cases, the hyphens were removed and the hyphenated compounds were turned to single words. These forms fall into three categories. In Category 1, the joined form was a Chinese first name. Chinese names (e.g. Li Dong Yuan) consisted of three characters with the first character ( $\mathrm{Li}$ ) being the family name, and the second (Dong) and third character (Yuan) being the first name (Dongyuan). Usually, the two characters of the first name were combined as Dongyuan. However, it is not uncommon that some writers of the textbooks used hyphens between the characters of the first names as Dong-yuan. In this case, Dongyuan and Dong-yuan were counted as two different lexical items by Range though they referred to the same person. The hyphens of people's names (e.g. Dong-yuan) were thus removed to keep all the names consistent.

In Category 2, the joined form of the hyphenated item appeared in the BNC/COCA base word lists. For example, the joint form of long-term, long-lasting and by-product can be found as longterm in BASEWRD 33, longlasting in BASEWRD 2, and byproduct in BASEWRD 11. Similarly, if a hyphenated item was made up of an affix and a word (e.g., re-establish) and the joined form can be found in the BNC/COCA base word lists (e.g., reestablish in BASEWRD 2), the hyphens were removed.

In Category 3, the joined form of hyphenated item satisfied Bauer and Nation's (1993) Level 6 criteria of making word families. If a hyphenated word consisted of an affix and a base word from BASEWRD 1 to 25 but the joined form was not in the relevant word list, the hyphen was also removed. For example, the joined form of pre-existing, preexisting, did not appear under the headword exist, but pre-existing was still turned to preexisting. This is because preexisting satisfied the criteria of word family which has been introduced in Section 3.4.3.3.

Although the vast majority of hyphens in hyphenated items were removed or replaced by spaces, hyphens in formulas like $R-C=O-R$ were kept. This decision was made because if the hyphens were replaced by spaces, the formulas which represented low-frequency but 
very specialized words $R-C=O-R$ would have become $R, C$ and $O$ which would then have been classified by the Range program as marginal words.

\subsubsection{Adapting the existing BNC/COCA base word lists}

In addition to the hyphenated words, another kind of 'Not in the lists' word types were proper nouns, abbreviations, and some low-frequency family members which have not been included in the existing BNC/COCA base word lists yet. The adaptation of the existing BNC/COCA base word lists was necessary for two reasons. First, the proper noun and abbreviation lists should be continually updated since each field of specialization has its own proper nouns and abbreviations. Second, some low-frequency family members might be missing if they do not appear in the BNC and COCA corpora used to develop the base word lists. This is acknowledged by Nation et al. (2016), who alerted us to the fact that the BNC/COCA word lists remain unfinished and low-frequency family members need to be continuously added to the existing word families. In the following subsections, how the base word lists were adapted will be described.

\subsubsection{Adapting the proper noun list}

As defined by Kennedy (2003), proper nouns are nouns which begin with a capital letter which form a separate class of nouns. Typical examples are "names of people, places, countries, days, months, institutions (e.g., the British museum), commercial products (e.g., a Cadillac), and holidays" (Kennedy, 2003, p. 147). Such proper nouns were pervasive in the TCM textbook corpora. If the words in 'Not in the lists' were proper nouns, they were added to the BASEWRD31 proper noun list. Danxi (name of people), Zhejiang (name of place), Fudan (name of institution) were instances in this case. In total, 2,795 proper nouns were added to the proper noun list (BASEWRD 31).

\subsubsection{Adapting the abbreviation list}

Abbreviations were formed from multiword sequences by taking the initial letter of each word in the sequence (Plag, 2003). There is a certain amount of medical abbreviations that occurred frequently in the TCM corpus in addition to the abbreviations listed in BASEWRD 34. Such abbreviations could not be ignored and were thus added to BASEWRD 34. For example, TCM (Traditional Chinese Medicine), SIDS (sudden infant death syndrome), $D K D$ (Diabetic kidney disease) were added to the abbreviations list. In total, 2,556 abbreviations were added to the abbreviation list (BASEWRD 34). 


\subsubsection{Adding family members to headwords in BASEWRD 1 to 25}

Some 'Not in the list' word types were low-frequency word family members. They were added to the word families in BASEWRD 1 to 25 of which it should have been a member. For example, preexisting should have been included as a family member under the headword exist in BASEWRD 2 because they were related in meaning and the affix pre appeared at Level 6 of Bauer and Nation's (1993) scale of making word families. In this case, preexisting was added to BASEWRD 2 under the headword exist. Likewise, other word types satisfied these two criteria were added to their headwords: (1) the meaning of the word types was related to the potential word families of which it might be a member; (2) the affix of the word types was in Bauer and Nation's (1993) scale from Level 2 to 6 . Other instances were pathophysiologic which was added to BASEWRD 14 as a family member of pathophysiology, and contraindicates which added to BASEWRD 22 under contraindicate. In total, 1,689 word types were added to the corresponding head words in BASEWRD 1-25.

\subsubsection{Categorizing special cases}

BASEWRD 32 is a base word list with marginal words including swear words, exclamations, and letters of the alphabet. It is surprising that BASEWRD 32 had a relatively high coverage (more than 1\%) over the TCM Corpora than what was reported by Nation (2012) in Wellington Written Corpus. Close inspection of the high-frequency lexical items in BASEWRD 32 showed that 11 items needed to be reclassified. For example, SOD was the abbreviation for superoxide dismutase in the TCM Corpora, but it appeared in BASEWRD 32. SOD was thus moved to the abbreviation list, and other cases were $A H A$, $D H I, E E, E H, E I, E R, G E E, H O, O C H$, and $O H$. Yang, which was grouped in the proper noun list, was indeed a family name in China. However, yang was an essential concept in TCM. In this case, yang was removed from the proper noun list and put into BASEWRD 35.

\subsubsection{Categorizing the remaining 'Not in the lists' word types}

When the TCM Corpora was processed and the existing BNC/COCA word lists were adapted, a large number of the 'Not in the list' word types have been categorized based on the word lists they belong. The remaining word types were by nature highly specialized technical words, which were used exclusively in the domain of TCM or by its related domains. 
In order to make these technical words comparable with the existing base word lists, they were categorized into two TCM base word lists in the same format as other base word lists. The loan words, for example $q i$ and $x u e$, borrowed from Chinese were separated from the other TCM words because they were a special group of vocabulary to the TCM learners especially when the learners are from different cultural and linguistic backgrounds. They might pose different learning burdens to learners from different linguistic and/or cultural backgrounds. The native Chinese-speaking learners may find the loan words easy because they have learnt these in Chinese; whereas the non-Chinesespeaking learners might find them extremely difficult to learn especially if they have no knowledge of Chinese. Thus, the loan words were separated from the other TCM technical words and were categorized into base word list 35 (BASEWRD 35), which is called the TCM loan word list. In addition to loan words, the other medical words which appeared in the 'Not in the lists' were grouped to TCM base word list 36 (BASEWRD 36). Since this word list mainly consists of general medical science words, it is called the TCM medical word list to differentiate from the TCM loan word list.

\subsubsection{Procedure}

After the steps mentioned above, the TCM Corpora were processed and the BNC/COCA base word lists were adapted with two TCM-specific base word lists added. The TCM Corpora were run on the Range program again, and thus the coverage figures of the BNC/COCA base word lists in the TCM Corpora were obtained. Part of the first research question (RQ1a) could then be answered. The remaining 'Not in the lists' words were then studied and categorized to answer Research Question 1b (RQ1b) as mentioned in Section 4.4.4.

To examine the coverage of high, mid, and low-frequency vocabulary in TCM texts, it is necessary to recall their definitions. Using Nation's (2012) frequency-based base word lists, Schmitt and Schmitt (2014) defined the first three 1,000 word families of the BNC/COCA lists (BASEWRD 1-3) as high-frequency vocabulary, those between 4,000 and 8,000 (BASEWRD 4-8) as mid-frequency vocabulary, and those above 9,000 (BASEWRD 9-25) as low-frequency vocabulary. The coverage of each frequency band was then calculated by adding the coverage of the BNC/COCA base word lists included in that frequency band. For example, the coverage of high-frequency vocabulary was determined 
by adding the coverage of BASEWRD 1,2 and 3. Accordingly, the coverage of mid and lowfrequency vocabulary was calculated. This analysis allows us to understand the TCM vocabulary from a broader perspective.

Vocabulary load refers to the number of 1000 -word-family lists needed by counting the lexical coverage of each BNC/COCA base word lists till the total coverage accumulated to 98\%. 98\% lexical coverage was used because it was regarded as the vocabulary necessary for optimal reading comprehension (Schmitt, Jiang, et al., 2011). The vocabulary load of the TCM Corpora was calculated by adding the coverage of BNC/COCA base word lists till the total coverage reached $98 \%$. To make this study comparable to other similar studies, the vocabulary load needed to reach $95 \%$ coverage was also counted which was considered as the minimum reading comprehension threshold (Laufer \& RavenhorstKalovski, 2010).

To determine the difference of vocabulary between TCM and WM, the WM Corpora were run over the Range (Heatley et al., 2002) with the adapted BNC/COCA base word lists (including the TCM base word lists). It was found that $3.70 \%$ of the tokens were not in the BNC/COCA 25,000 word lists. This percentage was fairly high. Therefore, it is worthwhile to have a look at those words which occur frequently in the medical corpus but not in any of the base word lists. For this reason, the 'Not in the list' words which occur more than 100 times in the WM Corpora were categorized according to the same principles for the TCM Corpora. The modified BNC/COCA base word lists and the WM Corpora were then run through the Range again and the results were thus obtained. As a result, WM lexical profile were obtained, and the differences between the TCM and WM vocabulary were determined.

To answer the research question about difference of vocabulary between TCM and general written English, the lexical profile of the Wellington Written Corpus were obtained directly from Nation (2012). The lexical profile of Wellington Written Corpus was chosen for two reasons. First, the Wellington Written Corpus is a general written corpus, so it allowed for a fair comparison with the TCM Corpora which is written. Second, it was chosen by Nation (2012) as the corpus to validate the BNC/COCA base word lists so that the coverage results of the BNC/COCA word lists over this written corpus were 
readily available. Thus, the comparison of lexical profile between the TCM and general written English could be carried out easily.

\subsection{Results}

The previous sections have described the corpus data collection, processing and analysis. This section focuses on presenting the results and findings of the analysis that addresses the research questions in turn.

\subsubsection{Lexical profile of the TCM texts along Nation's (2012) BNC/COCA lists}

The first research question concerns the lexical profile of TCM vocabulary along Nation's (2012) BNC/COCA base word lists. Table 4.7 presents the lexical profile of the TCM vocabulary along the frequency-based BNC/COCA base word lists with the number of word types, tokens, coverage and the most frequently used 3 words in each base word list provided.

As can be seen from Table 4.7, the TCM vocabulary was distributed widely across all the Nation's (2012) 25 BNC/COCA base word lists and four supplementary lists. The lexical coverage and word types provided by the first 1,000 to the twenty-fifth 1,000 word families are decreasing along the frequency-based BNC/COCA base word lists with a number of exceptions which were highlighted in bold font. The first three 1000 BNC/COCA word families accounted for a coverage of $56.11 \%, 10.46 \%$ and $8.74 \%$ of the total tokens in the TCM Corpora, respectively. Nonetheless, the coverage dropped dramatically to $3.86 \%$ by the fourth 1,000 word families and even less by the fifth and sixth base word lists with a reasonable number of word types involved. From the seventh 1,000 onwards, each additional 1,000 word-family added on only a small increase (less than $1 \%$ ) in coverage and involved a much smaller number of word types.

One interesting pattern observed from Table 4.7 is that the lexical coverage and word types provided by the first 1,000 to the twenty-fifth 1,000 word families decreased as might be expected but with a number of exceptions which were highlighted in bold. That is, some lower frequency base word lists provided higher coverage and involved more 
Table 4.7: Lexical profile of the TCM Corpora across BNC/COCA word lists

\begin{tabular}{|c|c|c|c|c|}
\hline BASEWRD & Types & Tokens & Coverage & Examples \\
\hline 1 & 4,048 & $1,951,763$ & $56.11 \%$ & the, and, of \\
\hline 2 & 3,706 & 363,977 & $10.46 \%$ & medicine, tongue, stomach \\
\hline 3 & 3,697 & 304,117 & $8.74 \%$ & patients, symptoms, clinical \\
\hline 4 & 2,234 & 134,360 & $3.86 \%$ & liver, deficiency, pulse \\
\hline 5 & 1,702 & 70,551 & $2.03 \%$ & kidney, abdominal, exterior \\
\hline 6 & 1,362 & 35,183 & $1.01 \%$ & bladder, nourish, vomiting \\
\hline 7 & 1,109 & 27,428 & $0.79 \%$ & glucose, hypertension, placebo \\
\hline 8 & 882 & 27,149 & $0.78 \%$ & tang, stagnation, cortex \\
\hline 9 & 685 & 23,318 & $0.67 \%$ & acupuncture, ling, constipation \\
\hline 10 & 605 & 22,668 & $0.65 \%$ & spleen, semen, wiry \\
\hline 11 & 553 & 16,264 & $0.47 \%$ & stasis, pathogenic, distension \\
\hline 12 & 390 & 9,926 & $0.29 \%$ & fu, lumbar, orifices \\
\hline 13 & 423 & 15,622 & $0.45 \%$ & phlegm, dong, disharmony \\
\hline 14 & 369 & 17,027 & $0.49 \%$ & yin, chai, apoptosis \\
\hline 15 & 328 & 4,918 & $0.14 \%$ & febrile, rhinitis, prolapse \\
\hline 16 & 335 & 6,446 & $0.19 \%$ & gallbladder, tian, wen \\
\hline 17 & 303 & 6,194 & $0.18 \%$ & dang, ducat, meta \\
\hline 18 & 281 & 4,438 & $0.13 \%$ & vacuity, enuresis, hui \\
\hline 19 & 204 & 6,932 & $0.20 \%$ & decoction, epigastric, auricular \\
\hline 20 & 214 & 3,228 & $0.09 \%$ & acupressure, costal, intercostal \\
\hline 21 & 170 & 1,629 & $0.05 \%$ & hemiplegia, axilla, dyspnea \\
\hline 22 & 139 & 1,528 & $0.04 \%$ & concha, astragalus, fluoxetine \\
\hline 23 & 140 & 2,275 & $0.07 \%$ & pericardium, hao, cornu \\
\hline 24 & 70 & 650 & $0.02 \%$ & malleous, malar, supraclavicular \\
\hline 25 & 90 & 1,280 & $0.04 \%$ & cocos, canthus, hyperglycemia \\
\hline 31Proper nouns & 4,421 & 93,041 & $2.67 \%$ & Chinese, Huang, Bai \\
\hline 32Marginal words & 24 & 31,180 & $0.90 \%$ & $\mathrm{G}, \mathrm{P}, \mathrm{L}$ \\
\hline $\begin{array}{l}\text { 34Transparent } \\
\text { compounds }\end{array}$ & 537 & 10,593 & $0.30 \%$ & headache, baseline, pathway \\
\hline 34Abbreviations & 2,961 & 78,361 & $2.25 \%$ & TCM, ST-36, SP-6 \\
\hline Total & 31,982 & $3,272,046$ & $94.07 \%$ & \\
\hline
\end{tabular}


word types than higher frequency base word lists. For example, the $13^{\text {th }} 1,000$ word families provided higher coverage and involved more word types than the $12^{\text {th }} 1,000$, while the $14^{\text {th }} 1,000$ had unusually higher coverage even than $11^{\text {th }} 1,000$ with fewer word types involved. This finding indicates that the $14^{\text {th }} 1,000$ word families might contain some words which were very frequently used in the TCM texts. The top two words yin and chai were cases in point (see Table 4.7). Yin is one of the essential concepts in the theory of TCM while chai is often seen in the names of herbal medicine. Due to the high occurrences of such technical lexis from some lower-frequency base word lists, it is not surprising that the distributional profile of TCM was different from that of general English as described by Nation (2012).

It is interesting to note that there were loan words from Chinese appearing in the BNC/COCA lists from Nation (2012). For example, yin is a loan word from Chinese which has become a well-known concept in English. According to the Merriam-Webster Dictionary (n.d.), yin is defined as 'the feminine passive principle in nature that in Chinese cosmology is exhibited in darkness, cold, or wetness and that combines with yang to produce all that comes to be'. There is no other homograph of yin defined in the dictionary. It is even more interesting to notice that some words in the BNC/COCA word lists actually represented loan words in this discipline rather than English words. For instance, chai is a loan word in TCM which is often used in the names of herbal medicine; however, it is also an English word defined as "a beverage that is a blend of black tea, honey, spices, and milk". The use of chai in TCM is illustrated as following.

Especially in combination with Chai $\mathrm{Hu}$, this herb directs other herbs to the ear. (TCM theory-based textbook corpus)

Similar cases were tang in the $9^{\text {th }} 1,000$ BNC/COCA lists, ling in the $9^{\text {th }} 1,000$, dong in the $13^{\text {th }} 1,000$. They could be either interpreted as an English word or a Chinese loan word depending on the contexts. Even though this kind of semiotic coincidence in English and Romanized Chinese is not very common, it might create confusion for learners especially native speakers of English. 
The four supplementary lists from Nation's (2012) BNC/COCA lists also provide interesting insights into TCM lexis. The coverage of the proper nouns over the TCM Corpora was $2.67 \%$. Typically, these nouns were the names of people (e.g., Dongyuan, $W u$ ) and places (e.g., Zhejiang, Fudan) in the TCM Corpora. The coverage of abbreviations in the TCM Corpora was $2.25 \%$. It means, comparatively speaking, the TCM texts used abbreviations extensively. The following paragraph is an example text from the journal article corpus with abbreviations underlined. It shows how abbreviations were frequently used in the TCM texts.

Liver fibrosis ( $\underline{\mathrm{LF}}$ ), as a result of wound healing response to recurrent liver injury, is thought to be an early reversible stage of liver cirrhosis. It is characterized by the formation of fibrotic scar tissue with abnormal accumulation of fibroblasts and myofibroblasts, and excessive synthesis and deposition of extracellular matrix (ECM) proteins. The development of antifibrotic therapy is important for patients with chronic liver diseases, especially for chronic hepatitis $B(\underline{\mathrm{HBV}})$ and $\mathrm{C}$ virus $(\underline{\mathrm{HCV}})$ infections, which are the most prevalent blood borne viral infection and the major causes of LF worldwide, especially in mainland China. Few $\underline{L F}$ treatments are effective and inexpensive without adverse side effect.

(TCM journal article corpus)

This example illustrates that there were two types of abbreviations. Some abbreviations (e.g., $H B V, H C V$ ) could be understood without knowing what each of the letters stand for. In other words, those abbreviations have become words in their own rights, and they were medical by nature. Other abbreviations (e.g., $L F, E C M$ ) were textual in that they mainly served to save space and make the written text easier to read.

Marginal words accounted for $0.90 \%$ of the total tokens. In the TCM Corpora, they were mainly letters of alphabet (e.g., $J, G$ ), most of which represent the initials in names of people (e.g., J in Akeson, J). Occasionally, some letters represented abbreviations such as $g$ for gram and $n$ for number. Both cases suggest that marginal words would not create much learning burden for the learners. Transparent compounds accounted for $0.30 \%$ of 
the total tokens in the TCM Corpora. The most common transparent compounds were headache, baseline, and pathway (see Table 4.7).

Taken together, the cumulative coverage of the 25,000 BNC/COCA word families and four TCM-adjusted supplementary lists (proper noun, marginal word, transparent compound and abbreviation lists) is $94.07 \%$ in the TCM Corpora. There were still $5.93 \%$ of the tokens which were not covered. In the next subsection, results about the remaining words which appeared in the 'Not in the lists' will be reported.

\subsubsection{TCM-specific vocabulary in TCM Corpora}

This part answers the question of what vocabulary remains beyond Nation's (2012) BNC/COCA base word lists and how can it be classified. Table 4.8 presents the lexical profile of two TCM base word lists, which were created by analyzing the 'Not in the lists' word types after adaptation of the BNC/COCA word lists. They were highly specialized words in this specialized discourse domain found in TCM texts but they did not appear in the BNC/COCA frequency-based and supplementary word lists.

\section{Table 4.8: Lexical profile of TCM base word lists}

\begin{tabular}{|l|l|l|l|l|}
\hline BASEWRD & Types & Tokens & Coverage & Examples \\
\hline 35TCM loan words & 1,261 & 114,285 & $3.29 \%$ & qi, yang, zi \\
\hline 360ther TCM words & 6,806 & 91,936 & $2.64 \%$ & radix, rhizoma, fructus \\
\hline Total & 8,067 & 206,221 & $5.93 \%$ & \multicolumn{1}{|l}{} \\
\cline { 1 - 4 } & &
\end{tabular}

The TCM loan word list (BASEWRD 35) contained only 1,261 word types (1,251 word families), which covered $3.29 \%$ of the total tokens. This is a rather high coverage, which is even higher than the fifth BNC/COCA base word list. Table 4.9 presents the main components of BASEWRD 35. It included all loan words that borrowed directly from Chinese, for example, terms about the essential concepts of TCM (e.g., qi, xue, yang). These loan words carried crucial information about the technical knowledge of TCM. In addition to essential concepts, the TCM loan word list also contained names of acupoints (e.g., Sishencong, Renying) and herbal medicine (e.g., Bai Shao, Chen Pi). They accounted for a large proportion of the word families in this list. These names were included in BASEWRD 
35 instead of BASEWRD 31 because they were content-bearing words which are of great importance in lexicalizing the subject knowledge of this specialized discourse community.

\section{Table 4.9: Examples of loan words from Chinese in the TCM corpora}

\begin{tabular}{ll}
\hline Components & Examples \\
\hline Essential concepts in TCM & qi, yang, wuxing \\
\hline Names of acupoints & Sishencong, Renying, Xiajuxu \\
\hline Names of herbs and herbal medicine & Baishao, Chenpi, Chaihu \\
\hline
\end{tabular}

The words in BASEWRD 35 might only be familiar to and used by the subject specialists in this discipline. They were essential terms which lexicalize the technical knowledge in this specialized discipline, and the importance of these words can also be highlighted by the high coverage they achieved.

BASEWRD 36 contained an overwhelming 6,806 word types (6,347 word families) and covers $2.64 \%$ of the total tokens. Typically, they were highly specialized words in TCM which are only familiar to experts in the field of healthcare or related fields (e.g., biology) and very unlikely to be met in everyday life. It can be Latin names of the herbal medicine (e.g., cinnamomi, rehmanniae), words that describe body or the function of body (e.g., acromioclavicular, thermoreceptors), words that describe disease (e.g., myositis, intussusception), and other words that are related to health care, medicine and TCM (e.g., $\mathrm{mmHg}$, unit of measurement for blood pressure). It is not surprising that many of them were of Latin origin, which are made up of regular affixes in medical science (e.g., acetyl-, amino ckemo-, gastro-, hepato-, hydroxy-, hyper-, hypo-, neuro-, pharmoco-), since Latin is the former lingua franca of medicine (Taavitsainen, 2006). This means that the words in BASEWRD 36 were to a large extent shared by TCM and other medical science disciplines. Furthermore, even though BASEWRD 36 contained 6,806 word types, 3,127 word types occurred only once in the whole TCM Corpus. This finding indicates that there was a great diversity in word choice in TCM, even though some of them were only used once.

The paragraph below is an example text from the practice-based textbooks with words from BASEWRD 35 underlined and words from BASEWRD 36 in bold. This example 
shows that the TCM texts can be loaded with Chinese names of the herbs from BASEWRD 35 and Latin names of the herbs from BASEWRD 36.

This condition is very chronic and occurs on a background of Spleen Yang deficiency and often Kidney Yang deficiency. In such cases one should add herbs to tonify Spleen and Kidney Yang such as Bai Zhu Rhizoma Atractylodis macrocephalae, Huang Qi Radix Astragali membranacei, Yin Yang Huo Herba Epimedii and Du Zhong Cortex Eucommiae ulmoidis.

(TCM textbook corpus)

To sum up, beyond Nation's (2012) BNC/COCA base word lists, TCM loan words in BASEWRD 35 and general medical science words in BASEWRD 36 made up the remaining vocabulary in TCM. In total, the two TCM base word lists had a coverage of $5.93 \%$. This figure means that at least one out of twenty words in the TCM texts was from the TCM base word lists, which highlights the importance of TCM-specific vocabulary in this discipline.

\subsubsection{Coverage of high, mid, and low-frequency vocabulary in TCM}

The second research question is related to the coverage of high, mid, and low-frequency vocabulary in relation to Schmitt and Schmitt's (2014) frequency bands, which allows us to look at the TCM vocabulary at a broader level. Table 4.10 presents the base word lists included in Schmitt and Schmitt's (2014) high, mid, and low-frequency vocabulary bands and their coverage over the TCM Corpora.

As illustrated in Table 4.10 , the high-frequency vocabulary contributed $75.31 \%$ of the total tokens to the overall coverage in the TCM Corpora, while the mid-frequency vocabulary contributed to a much smaller but still useful amount coverage of $8.47 \%$. The low-frequency vocabulary clearly provided the smallest proportion of coverage. In total, Nation's (2012) four supplementary lists (proper noun, marginal word, transparent compound and abbreviation lists) accounted for $6.12 \%$ of the running words in the TCM Corpora. This figure is fairly high mainly because of the high coverage of proper nouns and abbreviations in the TCM texts as explained in Section 4.5.1. The high coverage of the 
supplementary lists indicates that they play an important role in the written discourse community of TCM even though they might not pose much of a learning burden.

Table 4.10: Coverage of high, mid and low-frequency vocabulary in the TCM Corpora

\begin{tabular}{|l|l|l|}
\hline Frequency bands & Base word lists & Coverage \\
\hline High-frequency vocabulary (1,000-3,000) & BASEWRD 1, 2, 3 & $75.31 \%$ \\
\hline Mid-frequency vocabulary (4,000-8,000) & BASEWRD 4-8 & $8.47 \%$ \\
\hline Low-frequency vocabulary (9,000-25,000) & BASEWRD 9-25 & $4.17 \%$ \\
\hline $\begin{array}{l}\text { Proper nouns, marginal words, compounds, } \\
\text { abbreviations }\end{array}$ & BASEWRD 31-34 & $6.12 \%$ \\
\hline TCM-specific vocabulary & BASEWRD 35-36 & $5.93 \%$ \\
\hline Total & 31 & $100.00 \%$ \\
\hline
\end{tabular}

In addition, it is interesting to note that the TCM base word lists provided a cumulative coverage of $5.93 \%$ in the TCM Corpus, which is higher than the cumulative coverage of the low-frequency vocabulary band as a whole (4.17\%). Such result further highlights the importance of these two TCM base word lists, especially the lexical items with very high occurrences (e.g., qi, yang, rhizoma, fructus).

Further, due to the high coverage of these two TCM base word lists, it seems that Schmitt and Schmitt's (2014) definitions of mid and low-frequency vocabulary needed to be reconsidered in TCM. Table 4.11 presents Schmitt and Schmitt's (2014) framework of high, mid, and low-frequency vocabulary with corresponding base word lists provided in the second column. The framework represents the frequency levels of vocabulary in general English. Using the same framework, however, the third column of Table 4.11 presents the base word lists which should be included in each frequency level according to the coverage they achieved in the TCM Corpora. That is, if coverage was the criterion to decide the base word lists in each frequency band, the base word lists included in mid and low-frequency bands present a different picture in the discourse domain of TCM as shown in the third column of Table 4.11. 
Table 4.11: Base word lists in Schmitt and Schmitt's (2014) frequency bands and in the TCM Corpora

\begin{tabular}{|l|l|l|}
\hline $\begin{array}{l}\text { Frequency bands } \\
\text { vocabulary }\end{array}$ & Schmitt and Schmitt (2014) & In TCM Corpora \\
\hline $\begin{array}{l}\text { Mid-frequency } \\
\text { vocabulary }\end{array}$ & BASEWRD 4, 5, 6, 7, 8 & BASEWRD 1, 2, 3 \\
\hline $\begin{array}{l}\text { Low-frequency } \\
\text { vocabulary }\end{array}$ & $\begin{array}{l}\text { BASEWRD 9, 10, 11, 12, 13, } \\
14,15,16,17,18,19,20,21,\end{array}$ & $\begin{array}{l}\text { BASEWRD 7, 8, 9, 10, 14, 11, 13, } \\
12,19,16,15,18,20,23,21,22, \\
22,23,24,25\end{array}$ \\
\hline
\end{tabular}

Note: the base word lists were arranged according to coverage they achieve.

This analysis shows that high-frequency vocabulary worked well in the specialized texts; however, mid and low-frequency vocabulary had different importance in specialized texts due to the existence of frequently used technical words from low-frequency bands and from the TCM-specific base word lists. Even though the mid and low-frequency vocabulary did not fully capture the frequency patterns of vocabulary use in the specialized discipline of TCM, this does not undermine the value of frequency-based descriptions of vocabulary (Nation, 2012; Schmitt \& Schmitt, 2014). In the following subsection, the results of vocabulary load of the TCM texts will be reported.

\subsubsection{Vocabulary load of the TCM texts}

This part answers the questions about vocabulary load of English-medium texts along the BNC/COCA word-frequency scale. As mentioned in Section 4.2.2, TCM students usually start with the TCM theories and then proceed to clinical practices. Ideally, TCM students encounter the theory-based textbooks first, and then proceed to practice-based textbooks and journal articles. Therefore, it might be of pedagogical value to look at the vocabulary burden of the theory-based textbooks and practice-based textbooks separately. In addition, the journal articles represent another genre of materials that the TCM learners need to read at every stage of study. For this reason, the journal articles were analyzed separately from the textbooks. This section first reports on the vocabulary load of the TCM Corpora as a whole, then reports on different text types in the sequence of theory-based textbooks, practice-based textbooks and journal articles. 
Table 4.12 presents the cumulative coverage for the TCM texts as a whole and for theorybased textbooks, practice-based textbooks, and journal articles separately. Proper nouns, marginal words, transparent compounds, and abbreviations were counted on the amount of vocabulary necessary for reasonable comprehension for two reasons. First, they were indispensable due to their high coverage. Without the technical words in the TCM base word lists, the coverage number could never reach $98 \%$ coverage in such a highly specialized corpus. Nor could 95\% text coverage be reached. Second, these words were considered to have a minimal learning burden since the learners can easily recognize the name of a person or abbreviation from its spelling or they can easily understand the meaning of transparent compound from its parts (Nation et al., 2016).

Table 4.12: Cumulative coverage for the TCM texts

\begin{tabular}{|l|l|l|l|l|}
\hline BASEWRD & $\begin{array}{l}\text { TCM } \\
\text { Corpora }\end{array}$ & $\begin{array}{l}\text { Theory-based } \\
\text { textbooks }\end{array}$ & $\begin{array}{l}\text { Practice-based } \\
\text { textbooks }\end{array}$ & $\begin{array}{l}\text { Journal } \\
\text { articles }\end{array}$ \\
\hline $\begin{array}{l}\text { Proper nouns, marginal } \\
\text { words, transparent } \\
\text { compounds, abbreviations } \\
(31-34)\end{array}$ & $6.12 \%$ & $5.15 \%$ & $7.65 \%$ & $6.84 \%$ \\
\hline $\begin{array}{l}\text { TCM base word lists (35- } \\
36)\end{array}$ & $12.05 \%$ & $10.17 \%$ & $16.87 \%$ & $9.50 \%$ \\
\hline 1 & $68.16 \%$ & $69.12 \%$ & $70.91 \%$ & $64.75 \%$ \\
\hline 2 & $78.62 \%$ & $79.32 \%$ & $79.35 \%$ & $77.30 \%$ \\
\hline 3 & $87.36 \%$ & $86.89 \%$ & $86.15 \%$ & $89.00 \%$ \\
\hline 4 & $91.22 \%$ & $90.97 \%$ & $90.20 \%$ & $92.47 \%$ \\
\hline 5 & $93.25 \%$ & $93.27 \%$ & $92.51 \%$ & $93.97 \%$ \\
\hline 6 & $94.26 \%$ & $94.33 \%$ & $93.60 \%$ & $94.87 \%$ \\
\hline 7 & $\mathbf{9 5 . 0 5 \%}$ & $\mathbf{9 5 . 0 2 \%}$ & $94.36 \%$ & $\mathbf{9 5 . 7 8 \%}$ \\
\hline 8 & $95.83 \%$ & $95.82 \%$ & $\mathbf{9 5 . 3 3 \%}$ & $96.36 \%$ \\
\hline 9 & $96.50 \%$ & $96.43 \%$ & $95.93 \%$ & $97.15 \%$ \\
\hline 10 & $97.15 \%$ & $97.14 \%$ & $96.79 \%$ & $97.55 \%$ \\
\hline 11 & $97.62 \%$ & $97.68 \%$ & $97.30 \%$ & $97.91 \%$ \\
\hline 12 & $97.91 \%$ & $97.97 \%$ & $97.65 \%$ & $\mathbf{9 8 . 1 3 \%}$ \\
\hline 13 & $\mathbf{9 8 . 3 6 \%}$ & $\mathbf{9 8 . 3 7 \%}$ & $\mathbf{9 8 . 2 5 \%}$ & $98.49 \%$ \\
\hline
\end{tabular}

For these reasons, the coverage figures of proper nouns, marginal words, transparent compounds, abbreviations, and TCM base word lists were counted in this study until the cumulative percentage arrived at $95 \%$ and $98 \%$ coverage. To make the figures easier to read, BASEWRD 31-36 were placed at the top of Table 4.12 . The base word lists after $98 \%$ 
coverage were not included since they were not the focus here and it could also make the table easier to read (see Appendix 1 for Table 4.12 including coverage of all base word lists).

As highlighted in bold in Table 4.12, to reach the 95\% and 98\% coverage level of the TCM texts overall, a vocabulary of the most frequent 7,000 and 13,000 word families respectively were needed from the BNC/COCA base word lists plus proper nouns, marginal words, transparent compounds, abbreviations, and TCM base word lists. Knowledge of the most frequent 7,000 word families plus proper nouns, marginal words, transparent compounds, abbreviations, and TCM base word lists was needed to reach 95\% coverage level of the theory-based textbooks. The corresponding figures for practicebased textbooks and journal articles were 8,000 and 7,000 word families respectively. To reach $98 \%$ coverage level, larger vocabulary sizes were need: 13,000 word families for theory-based textbooks and also for practice-based textbooks, while 12,000 word families for journal articles. The results indicated that at 95\% coverage, practice-based textbooks were the most lexically demanding, meaning the learners need a larger vocabulary size to start with the practice-based textbooks. At $98 \%$ coverage, it is surprising that journal articles were the least lexically demanding.

In sum, the results indicated that, along the BNC/COCA word-frequency scale, TCM texts had a rather heavy vocabulary burden. The TCM learners not only need vocabulary at different frequency levels but also proper nouns, marginal words, transparent compounds, abbreviations, TCM specific vocabulary from the TCM loan word list and general medical word list to attain a reasonable comprehension of the textbooks and journal articles in this discourse community. This means English-medium TCM texts used a diversity of vocabulary to construct the technical knowledge of such a specialized discipline. In the next section, the lexical profile of the WM Corpora along Schmitt and Schmitt's (2014) vocabulary framework will be presented in comparison with that in TCM.

\subsubsection{Comparison of lexical profile in TCM and Western Medicine}

The fifth research question relates to the difference of lexical profile between TCM and Western medicine (WM). Table 4.13 presents the lexical profile of WM Corpora along 
high, mid, and low-frequency vocabulary bands in comparison with that of the TCM Corpora. Due to the large amount of technical vocabulary, medical abbreviations and proper nouns in the WM Corpora, $2.21 \%$ of the running words were not covered by the adapted BNC/COCA base word lists as shown in Table 4.13. Despite this, the results can still provide valuable information from a lexical profile perspective if they were considered as preliminary.

Table 4.13: Comparison of high-, mid-, and low-frequency vocabulary in WM Corpora and TCM Corpora

\begin{tabular}{|l|l|l|}
\hline Frequency bands & TCM Corpora & WM Corpora \\
\hline $\begin{array}{l}\text { High-frequency vocabulary } \\
(1,000-3,000)\end{array}$ & $75.31 \%$ & $76.07 \%$ \\
\hline $\begin{array}{l}\text { Mid-frequency word vocabulary } \\
(4,000-8,000)\end{array}$ & $8.47 \%$ & $9.80 \%$ \\
\hline $\begin{array}{l}\text { Low-frequency vocabulary } \\
(9,000-25,000)\end{array}$ & $4.17 \%$ & $5.76 \%$ \\
\hline $\begin{array}{l}\text { Proper nouns, marginal words, } \\
\text { compounds, abbreviations }\end{array}$ & $6.12 \%$ & $4.17 \%$ \\
\hline BASEWRD 35 loan words & $3.29 \%$ & $0.00 \%$ \\
\hline BASEWRD 36 medical words & $2.64 \%$ & $1.99 \%$ \\
\hline Not in the list & $0.00 \%$ & $2.21 \%$ \\
\hline Total & $100 \%$ & $100.00 \%$ \\
\hline
\end{tabular}

As can be seen from Table 4.13, both TCM (75.31\%) and WM (76.07\%) texts were similar in using relatively small proportions of general high-frequency vocabulary. It is interesting that the mid and low-frequency vocabulary provided a slightly higher coverage over the WM texts than the TCM texts. To be specific, the mid-frequency vocabulary provided a $1.33 \%$ higher coverage in the WM texts than the TCM texts, whereas the low-frequency vocabulary provided a more visibly higher coverage of $1.59 \%$ in the WM texts than the TCM texts. These results mean that the TCM texts make slightly less use of vocabulary from mid, and low-frequency bands compared to the WM texts. 
In contrast, the supplementary word lists (proper nouns, marginal words, compounds, and abbreviations) provided a 1.95\% higher percentage in TCM than in WM. This result is understandable considering that the proper noun and abbreviation lists were specifically adapted to the TCM Corpora. Nonetheless, $1.95 \%$ higher is still a noting figure. It is not surprising that the TCM loan word list (BASEWRD 35) had a coverage of 3.29\% over the TCM texts whereas these words covered $0 \%$ of the WM texts. This result confirms that the TCM loan word list is a highly specialized word list exclusively used in the discipline of TCM. It is also worth noticing that BASEWRD 36 covered $1.99 \%$ of the running words in the medical corpus, which is slightly lower than that in the TCM texts. This result confirms that BASEWRD 36 contained a large amount of technical words which were shared by WM and TCM. Cytokines, hypothalamic, leukocyte and splenomegaly are cases in point.

To sum up, the results show that the TCM and WM texts had slightly differences in terms of the use of high, mid, and low-frequency vocabulary, and the general medical words in BASEWRD 36. The differences in the use of lexical items in the four supplementary lists are noting. What deserves more attention is the difference in the use of loan words, which indicates that BASEWRD 35 is a unique word list used only in TCM. Together, the results validate my observation that it is not suitable to apply the findings of medical vocabulary to the TCM learners since the medical word lists may not be able to address their lexical needs.

\subsubsection{Comparison of lexical profile in TCM and general written English}

The sixth research question is about the difference of lexical profile between TCM and general written English. Table 4.14 presents the coverage of high, mid, and low-frequency vocabulary in TCM Corpora and that in Wellington Written Corpus (Nation, 2012) using Schmitt and Schmitt's (2014) frequency band as a unit. It needs to be clarified here that 'Not in the lists' words in the TCM Corpus have been categorized into two TCM base word lists while they were not processed in Wellington Written Corpus. Therefore, in the TCM Corpora, the coverage of 'Not in the lists' vocabulary means the cumulative coverage of the two TCM base word lists (BASEWRD 35 and 36) as reported in Section 4.5.2. 
Table 4.14: Comparison of high-, mid-, and low-frequency vocabulary in TCM Corpora and in Wellington Written Corpus

\begin{tabular}{|l|l|l|}
\hline Frequency bands & TCM Corpora & Wellington Written Corpus \\
\hline $\begin{array}{l}\text { High-frequency vocabulary } \\
(1,000-3,000)\end{array}$ & $75.31 \%$ & $89.35 \%$ \\
\hline $\begin{array}{l}\text { Mid-frequency vocabulary } \\
(4,000-8,000)\end{array}$ & $8.47 \%$ & $4.38 \%$ \\
\hline $\begin{array}{l}\text { Low-frequency vocabulary } \\
(9,000-25,000)\end{array}$ & $4.17 \%$ & $1.14 \%$ \\
\hline $\begin{array}{l}\text { Proper nouns, marginal words, } \\
\text { compounds, abbreviations }\end{array}$ & $6.12 \%$ & $3.86 \%$ \\
\hline 'Not in the lists' vocabulary & $5.93 \%$ & $1.25 \%$ \\
\hline Total & $100.00 \%$ & $100.00 \%$ \\
\hline
\end{tabular}

Note: 'Not in the list' vocabulary in the TCM Corpora means the TCM-specific vocabulary.

As illustrated in Table 4.14, the coverage of high-frequency vocabulary in the TCM Corpus (75.31\%) was much lower than that in the Wellington Written Corpus (89.35\%). This means that general written English relied much more heavily on the general highfrequency words than the specialized texts of TCM. In contrast, mid-frequency vocabulary witnessed a reverse trend with a considerably higher coverage in the TCM Corpora $(8.47 \%)$ than the Wellington Written Corpus (4.38\%). The similar trend appeared in the low-frequency vocabulary, which provided $3.03 \%$ higher coverage in the TCM Corpora than the Wellington Written Corpus. The much lower coverage of highfrequency vocabulary coupled with considerably higher coverages of the mid and lowfrequency vocabulary in the TCM Corpora indicates that TCM is a lexically demanding discipline.

Similarly, Nation's (2012) four supplementary lists (proper noun, marginal word, transparent compound and abbreviation lists) accounted for $6.12 \%$ of the running words in the TCM Corpora, which is $2.26 \%$ higher than that of Wellington Written Corpus. This result is understandable since the TCM texts used a very large amount of abbreviations as explained in Section 4.5.1. In addition, compared to Wellington Written Corpus, much more vocabulary in the TCM Corpora was not covered by the high, mid, and low- 
frequency vocabulary, and thus has been categorized into two TCM word lists. In other words, the TCM texts used a much larger set of specialized words than general English texts. This result further reinforces the view that TCM vocabulary is much more challenging than vocabulary in general written English texts.

In sum, the general picture emerging from the above analysis is that TCM had a very different lexical profile from that of the general written English. The TCM texts used a much smaller proportion of high-frequency vocabulary while a larger proportion of mid, low-frequency vocabulary and items in the four supplementary word lists. Moreover, the TCM texts used a wide range of words that laid outside the aforementioned word lists. Such results suggest that TCM vocabulary is very challenging.

\subsection{Summary of the findings and rationale for next study}

In this chapter, the lexical profile of the TCM Corpora over the adapted BNC/COCA base word lists provided us a useful insight into the nature of vocabulary in TCM. It showed that TCM vocabulary was distributed across all the twenty-five 1,000 BNC/COCA word family lists, the four supplementary base word lists, and the two TCM base word lists. Another important finding is that the BNC/COCA base word lists were not ordered in the same way in the specialized TCM Corpora as that in the general English due to the high occurrences of some technical word in some lower-frequency base word lists. Further, both TCM base word lists - BASEWRD 35 and 36 - had higher coverage than the fifth BNC/COCA base word lists, suggesting that the mid and low-frequency vocabulary had a different picture in this specialized discourse domain if off-list TCM technical vocabulary was taken into consideration. In Chapter 7, these findings will be drawn together with other findings of the present study to discuss the value of general high, mid and lowfrequency vocabulary in TCM (Section 7.5.1).

In addition, it was found that the TCM learners have a rather heavy vocabulary learning burden with a vocabulary load of 13,000 and 7,000 word families plus proper nouns, marginal words, transparent compounds, abbreviations, and TCM base word lists at 98\% and 95\% coverage level. These figures mean that TCM is a very lexically demanding discipline. Further, it is important to find that TCM journal articles had a less vocabulary burden than the textbooks at the $98 \%$ coverage level. The comparison of TCM and WM 
vocabulary showed they had different lexical profiles with the most striking difference being the use of loan words in the TCM texts. The comparison of vocabulary in TCM and general written English showed that TCM vocabulary is much more challenging than that of the general written English in that it used a much smaller set of general high-frequency words. All these findings suggest that TCM vocabulary is of great complexity and challenge. These findings will be used in Section 7.5.2 to demonstrate the value of vocabulary load analysis in understanding vocabulary in specialized disciplines.

Overall, the two TCM base word lists (BASEWRD 35 and 36) played a crucial role in constructing the technical knowledge in the highly specialized discipline of TCM in that they accounted for $5.93 \%$ of the total tokens in the TCM Corpora. That is about one out of twenty words in a line of TCM texts, which is much less technical word dense than what was found by Chung and Nation (2003). They reported that one out of three words in the anatomy texts is technical vocabulary. This is because a large amount of the technical vocabulary was included in BASEWRD 1-25, which has not been identified yet. Hence, Study 2 further investigates technical vocabulary in the first to twenty-fifth BNC/COCA base word lists. It also involves the development of a TCM technical word list including technical words in and beyond the BNC/COCA base word lists, and the development of a technical vocabulary list of multiword units. 


\section{Chapter 5 Technical vocabulary in Traditional Chinese Medicine}

\subsection{Introduction}

In the previous chapter, a comprehensive lexical profile analysis of textbooks and research articles in Traditional Chinese Medicine (TCM) has been carried out. All lexical items in the TCM Corpora were taken into account. This process involved profiling the TCM vocabulary along the BNC/COCA base word lists and examining words which did not belong to any of the BNC/COCA base word lists and supplementary lists. These words were then categorized into two TCM base word lists. The present study (henceforth Study 2 ) has three goals. The first goal is to use a keyword analysis and a semantic rating scale to identify technical vocabulary in frequency and range-based 1-25 BNC/COCA base word lists (BASEWRD 1-25). Then, these technical words will be further investigated in terms of their semantic characteristics. The second is to develop a TCM technical word list based on word types, and the third aim is to develop a technical vocabulary list of multiword units. To differentiate technical vocabulary of single and multiword units, the term technical word(s) refers to technical vocabulary of single word units while technical lexical bundle(s) refers to two to five multiword units. Specifically, this chapter aims to answer the following research questions (RQs):

1. Which lexical items in Nation's (2006) 1-25 frequency-based BNC/COCA word lists are technical words in the specialized discipline of TCM?

1a. How many of them are from the high, mid, and low-frequency vocabulary bands, respectively?

1b. What are the semantic characteristics of those technical words?

2. What is the overall coverage of technical vocabulary in the TCM texts?

3. Which technical words are frequently used in TCM so that they can be included in the TCM technical word list?

4. Is there a difference in the coverage of TCM technical word list in theory-based textbooks, practice based textbooks and journal articles?

5. What is the coverage of the TCM technical word list in an independent collection of TCM texts, in the medical texts and general written texts? 
6. Which technical lexical bundles are frequently used in the specialized discipline of TCM?

Using the TCM Corpora built in the previous study (see Section 4.2 for details), this chapter focuses on describing methodology used in Study 2 and reporting results to answer the research questions listed above. Specifically, Section 5.2 to 5.4 describe the methods used in corpus analysis to identify technical words from BASEWRD 1-25, to develop a technical word list, and a lexical bundle list in the TCM texts. They are then followed by Section 5.5 which reports the results and findings of the analysis with answers to the research questions. This chapter will be closed with a brief summary of the important findings, and implications for the next study (Section 5.6).

\subsection{Identifying technical words in 1-25 BNC/COCA base word lists}

While conducting the lexical profile analysis (see Chapter 4), two TCM base word lists were created including words (e.g., qi, rhizome, moxibustion) which did not appear in any of the BNC/COCA word lists. Along with these clearly technical words, there was also a huge number of technical words which were included in the first to twenty-fifth BNC/COCA base word lists (BASEWRD 1-25). Heart, treatment, and syndrome were cases in point. These words are essential in the construction of disciplinary knowledge in TCM. To identify technical words in the BASEWRD 1-25, this study adopted a method which combines quantitative analysis of keywords and qualitative analysis with the guidance of pre-determined semantic rating scale. The keyword analysis was used to identify words which are potential candidates of technical vocabulary based on the assumption that technical words occur more frequently in the technical texts than that in the general English texts (Chung, 2003; Scott, 2006; Yang, 1986). The qualitative analysis was used to decide whether words from the keyword analysis are truly technical vocabulary.

In the following subsections, both the methods of identifying technical words and the procedures are described in detail. This description includes deciding on the unit of analysis, selecting a comparison corpus to prepare for the keyword analysis, conducting the keyword analysis, adapting a semantic rating scale for qualitative analysis, and analyzing the semantic features of those technical words. 


\subsubsection{Deciding unit of counting}

In this study, the unit of analysis in identifying technical words was word type because research evidence has shown that word type is the most suitable unit for counting highly specialized vocabulary (Chung \& Nation, 2004; Coxhead et al., 2016; Ha, 2015; Nation, 2013; Nation et al., 2016; Ward, 2009). First, not every word family member is a technical word in specialized disciplines. For example, Coxhead et al. (2016) found that fixing is a technical word in Carpentry while fix is not. Second, some members in a word family may not occur at all or occur with low frequency in the specialized corpora. Ha (2015) found that hierarchy is a very prominent technical word in Finance while hierarchical and hierarchies are not. Last, it has been noted by researchers that knowledge of one form of a word does not necessarily imply knowledge of its inflections or derivations (Durrant, 2013; Martinez \& Murphy, 2011; Ward, 2009). For example, Martinez and Murphy (2011) argued that there is little reason to suppose learners can derive the meaning of a word like fishy (meaning not completely right, honest, or legal) from fish given the semantic distance between them. For these reasons, this study decided to use word type as the unit of counting words.

\subsubsection{Developing the comparison corpus for keyword analysis}

The comparison corpus was developed to conduct the keyword analysis, which was used to identify potential technical words in the target TCM Corpora. Table 5.1 shows that the comparison corpus was comprised of a roughly equal number of fiction, magazine and newspaper texts. They represented different kinds of general written English. The present study chose general written English corpus as the comparison corpus for two reasons. First, most previous studies used a comparison corpus with general English texts (Chung, 2003; Chung \& Nation, 2004; Scott, 1997). Nonetheless, this study preferred written texts over spoken texts because the target corpora (TCM Corpora) are written. Excluding spoken texts can thus ensure that the keywords generated are not an endless list of written-text specific words because research has shown that spoken and written texts are very different from a vocabulary perspective (Dang, 2017; Dang \& Webb, 2014; van Zeeland \& Schmitt, 2013). Second, a general written English corpus could help achieve the aim of finding out potential candidates which are likely to be technical words as a basis of qualitative analysis of semantics (see Section 5.2.4). 
Table 5.1: Overview of the comparison corpus

\begin{tabular}{ll}
\hline Materials & Tokens \\
\hline Fiction & $5,980,535$ \\
Magazine & $5,745,610$ \\
Newspaper & $5,624,990$ \\
\hline Total & $17,351,135$ \\
\hline
\end{tabular}

Together, the comparison corpus consisted of 17,351,135 running words, which is about five times larger than the target TCM Corpora (see Section 4.2 for details). This number was decided because the present study adopted the view that the larger the comparison corpus the better (Chung, 2003; Scott, 2009, 2010). This view is also in line with the suggestion proposed by Chung (2003) that a comparison corpus needs to be big enough to allow all general high-frequency vocabulary to appear.

All texts in the comparison corpus were obtained from the Corpus of Contemporary American English (COCA) (Davies, 2008-). COCA was chosen as the source because it is one of the largest and updated general English corpora available at the time of writing. COCA contains more than 520 million tokens, and it has been divided into spoken, fiction, magazines, newspapers, and academic texts with similar sizes. Another advantage of COCA (Davies, 2008-) is that the corpus can be separated in terms of genres and the texts are available in TEXT files. As a result, a roughly equal number of fiction, newspaper and magazine texts were downloaded from the corresponding sections of the COCA. When the comparison corpus was developed, the next step is to conduct the keyword analysis, which is the focus of the next section.

\subsubsection{Conducting the keyword analysis}

The keyword analysis was used to find out keywords in the TCM Corpora. As defined by Scott (1997), keywords are words which occur with unusual frequency in a target corpus by comparison with a comparison corpus. Such keywords are highly likely to be the technical vocabulary because technical words often occur more frequently in specialized texts than in general use (Scott, 2006). The software program - Wordsmith Tools 6.0 (hereafter Wordsmith) (Scott, 2012) was employed for the keyword analysis since it can automatically compute the keyness of a word in a target corpus in comparison to a 
comparison corpus. It involves three stages (Scott, 2012). The first stage is to generate a word list with all word types and their frequencies for the comparison corpus using the WordList function in Wordsmith. The second stage is to generate a same sort of word list with the target specialized corpus WordList function. The last stage is to compare the two word lists generated in stage one and two using the KeyWords function in Wordsmith and identify keywords in target specialized corpus. A keyword list can thus be created with all potential technical words, which are ordered according to their relative keyness.

Before carrying out the keyword analysis, a pilot study with one TCM textbook corpus The foundations of Chinese medicine (Maciocia, 2005) was conducted to ensure that the TCM Corpora and the comparison corpora could be processed appropriately by Wordsmith and to see if the comparison corpus was reliable. Since The foundations of Chinese medicine only contained 487,968 tokens, the comparison corpus was reduced to $2,500,000$ words proportionately in fiction, magazine and newspaper to make the comparison corpus five times larger than the pilot specialized corpus. Following the steps mentioned above, a pilot TCM keyword list was created with the top 20 key words presented in Table 5.2.

\section{Table 5.2: Top 20 keywords of TCM Corpora from the pilot keyword analysis}
1. treatment
11. kidney
2. yin
12. patients
3. blood
13. phlegm
4. liver
14. and
5. deficiency
15. Chinese
6. acupuncture
16. symptoms
7. pain
17. medicine
8. heat
18. points
9. spleen
19. tongue
10. of
20. clinical

One problem identified when conducting the pilot study was that, as illustrated in Table 5.2, function words (e.g., of, and) and proper nouns (e.g., Chinese) also appeared in the pilot keyword list though most of the words were technical words (e.g., treatment, yin). 
To prevent these obviously not technical words from appearing in the keyword lists, stop lists were attached in the KeyWords function. Stop lists are lists of words, such as function words, that the computer program was instructed not to take into account when performing the lexical analysis (Chung \& Nation, 2004). One major advantage of using stop lists was that all the words in the stop lists could be excluded at once.

In this study, the function word list from the Range program (Heatley et al., 2002), the proper noun list (BASEWRD 31), the marginal word list (BASEWRD 32) and the abbreviation list (BASEWRD 34) were adopted as stop lists. The TCM word lists (BASEWRD 35 and 36) were also used as stop lists because these words had already been classified as technical words (see Chapter 3 for details) and the aim of the present study is to identify technical words from BASEWRD 1-25. Therefore, it was not necessary to include words from BASEWRD 31 to 36.

After applying the stop lists, the problem identified in the pilot study was solved. The keyword analysis was then conducted. The whole TCM Corpora and the whole comparison corpus were processed by the WordList function. A word list with TCM vocabulary and a comparison word list with general English vocabulary were developed from this analysis. These two word lists were compared by the KeyWords function (with the above mentioned stop lists) and the TCM keyword list was thus generated. The TCM keyword list consisted of 4,982 word types which were potential technical words in TCM.

\subsubsection{Developing the semantic rating scale}

The keyword analysis generated a list of potential technical words in TCM, but not all these items were technical in nature. Clearly, non-technical words, such as therefore and due, were scattered across the keyword list. Therefore, a qualitative analysis was used to decide whether they were truly technical words or not. To ensure that the qualitative analysis was valid and systematic, a semantic rating scale was developed to draw a line between technical and non-technical words with meaning being the only criterion as shown in Table 5.3. The semantic rating scale was guided by that of Chung and Nation (2003, p. 254), which was originally developed to identify technical words in anatomy by classifying words on the closeness of the meaning of a word to anatomy (See Section 2.3.2 for more details about the rating scale). Since the present study did not intend to 
distinguish the level of closeness of a word to the specialized discipline, the semantic rating scale was simplified. It contained only two scales - the non-technical scale and the technical scale with four parts, as shown in Table 5.3.

Table 5.3: Overview of the semantic rating scale

\begin{tabular}{|l|l|l|}
\hline Scales & Description & Examples \\
\hline Non-technical & $\begin{array}{l}\text { has meaning that has no particular } \\
\text { relationship with TCM }\end{array}$ & due, summary, chapter, box \\
\hline \multirow{5}{*}{ Technical } & $\begin{array}{l}\text { 1. loan word and the meaning is } \\
\text { related to TCM }\end{array}$ & fu, yin, chai, wan \\
\cline { 2 - 3 } & $\begin{array}{l}\text { 2. has a meaning related to TCM and } \\
\text { is (almost) the same as the general } \\
\text { meaning }\end{array}$ & $\begin{array}{l}\text { sweating, pain, efficacy, } \\
\text { function, external, lateral, } \\
\text { group }\end{array}$ \\
\cline { 2 - 3 } & $\begin{array}{l}\text { 3. has a meaning related to TCM } \\
\text { but is different from the general } \\
\text { meaning }\end{array}$ & $\begin{array}{l}\text { tissue, message, liver, } \\
\text { course, back }\end{array}$ \\
\cline { 2 - 3 } & $\begin{array}{l}\text { 4.has a meaning related to and is only } \\
\text { related to TCM or general medical } \\
\text { science }\end{array}$ & $\begin{array}{l}\text { moxibustion, acupuncture, } \\
\text { apoptosis }\end{array}$ \\
\hline
\end{tabular}

The adapted scale has three points of difference from that of Chung and Nation (2003). The first major difference is Part 1 in the technical scale (in italics) in Table 5.3 about loan words. Due to the special cultural origin of TCM, a large amount of loan words was used to lexicalize the technical knowledge (see Section 5.4.2 for more details). The second major difference is Part 2 in the technical scale (underlined) in Table 5.3. If using Chung and Nation's (2003) scale, this group of words would be classified as Step 2, meaning they were minimally related to the specialized discipline. Unfortunately, Chung and Nation (2003) did not provide information about why they considered them as non-technical. This group of words was considered as technical in this study because they provided support in the construction of subject knowledge even though they might not create the essential subject concepts. For example, group often referred to participants in the health and medical community usually with a connotation of patients, and thus was considered 
to be a technical word. A second difference is that Part 3 in the technical scale (in bold) was not mentioned in the rating scale of Chung and Nation (2003).

\subsubsection{Applying the semantic rating scale and analyzing the semantic characteristics of technical words}

Every word in the TCM keyword list was checked against the semantic rating scale manually. This process involved checking the meaning of a word using multiple dictionaries such as Merriam-Webster Online Dictionary and Macmillan Dictionary, and confirming the meaning by consulting the concordance lines in the TCM Corpora or using my subject knowledge of TCM. One advantage of the present research is that, as the researcher, I have a Bachelor's degree in TCM, which enables me to bring in subject knowledge when making a decision. The words rated as non-technical were removed from the keyword list. After removing all non-technical words, a list of 4,368 TCM technical words from BASEWRD 1 to 25 were identified.

When the technical words from the frequency-based BNC/COCA word lists were identified, those items were then investigated in terms of their semantic characteristics. Specifically, the semantic characteristics of technical words identified from the 1-25 BNC/COCA word lists were analyzed according to Figure 5.1, which is a framework for analyzing the semantic features of TCM technical words. In this way, a better understanding of these technical words can be obtained. In the next section, how the TCM technical word list is developed will be described in detail.

\subsection{Developing the TCM technical word list}

Study 1 identified 8,607 technical words in TCM outside the BNC/COCA base word lists, while the first part of the present study identified 4,368 technical words in the 1-25 frequency-based BNC/COCA base word lists. This means that there is a huge repertoire of technical words in TCM. To develop a TCM technical word list of pedagogical use in the discipline of TCM, the next steps are to establish the characteristics for this list (Section 5.3.1) and set the criteria for the items to be included in this list (Section 5.3.2). Section 5.3.3 describes how the items of the TCM technical word list were selected and the TCM technical word list was validated. The last section (Section 5.3.4) provides detailed specifications for the TCM technical word list as a brief overview of the word list developed from the present study. 
Figure 5.1: Framework for analyzing semantic features of TCM technical words

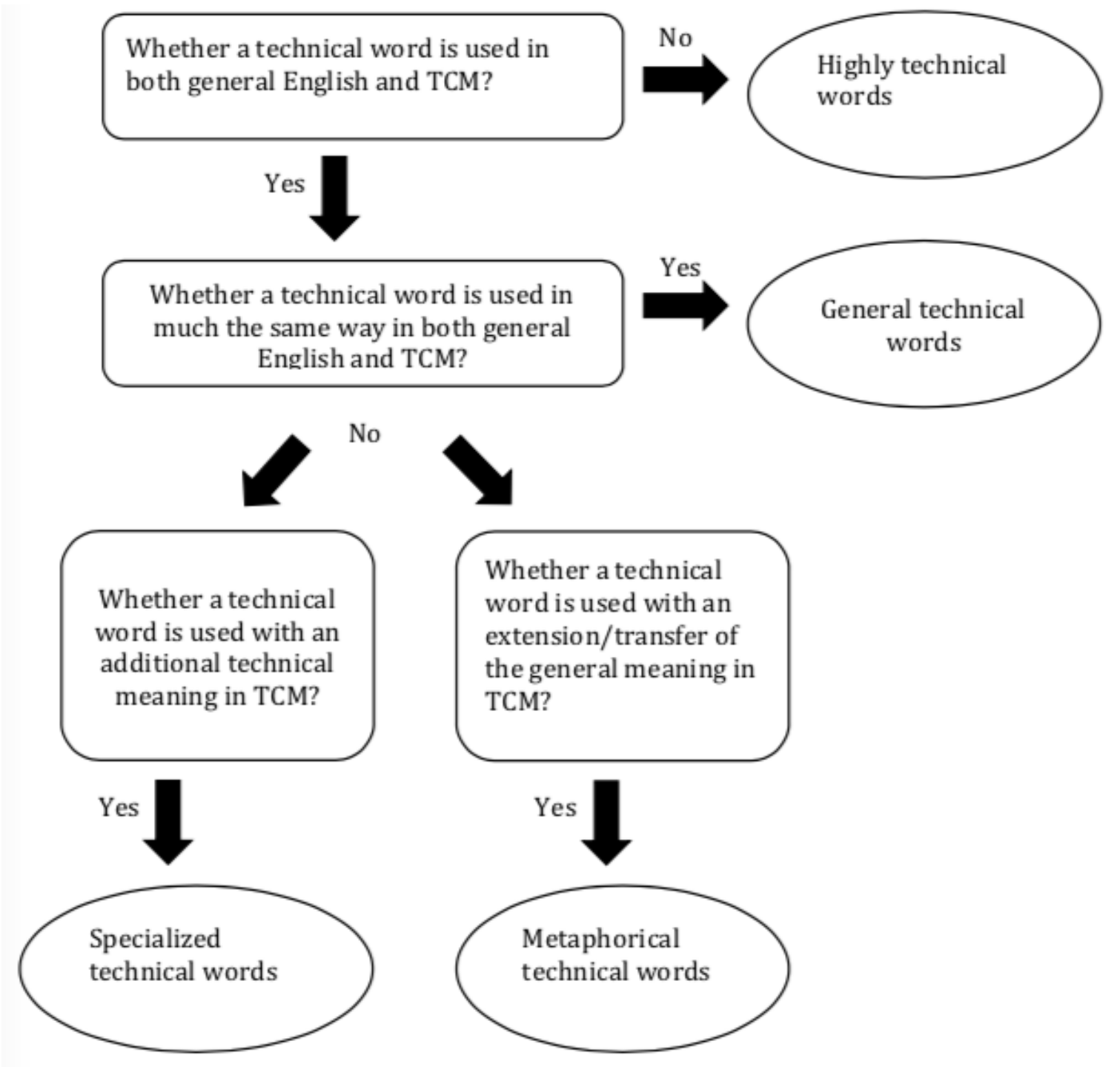




\subsubsection{Establishing the characteristics for the TCM technical word list}

The TCM technical word list should possess the following three characteristics:

1. Technical meaning: the TCM technical word list must be technical by nature and should take all kinds of words that have a technical meaning into consideration since they are all necessary vocabulary for developing TCM concepts.

2. Size: the TCM technical word list must contain a reasonable number of lexical items for pedagogical use. Therefore, the technical word list should contain no more than 3,000 word types as a word list with more than 3,000 word types will be too demanding for the learners. The items in the list can then be divided into three sub-lists according to their occurrences in the TCM Corpora (see Table 5.4) to make the TCM technical word list into different sets of smaller attainable goals.

\section{Table 5.4: Sub-lists of the TCM technical word list}

\begin{tabular}{|l|l|}
\hline Sub-list & Components \\
\hline Sub-list 1 & The most frequent 1,000 word types in the TCM Corpora \\
\hline Sub-list 2 & The most frequent 1,001 -2,000 word types in the TCM Corpora \\
\hline Sub-list 3 & Outside the most frequent 2,000 word types in the TCM Corpora \\
\hline
\end{tabular}

3. Adaptability to learners' linguistic background and vocabulary levels: each TCM technical vocabulary sub-list should be divided into three levels based on Nation's (2012) BNC/COCA base word lists to benefit learners from different linguistic backgrounds and vocabulary levels as shown in Table 5.5.

As can be seen from Table 5.5, Level 1 contains TCM technical words from the first to third 1,000 BNC/COCA frequency levels, namely, words from high-frequency vocabulary band. Level 2 contains technical words outside the first to third 1,000 BNC/COCA levels while Level 3 contains loan words. The division was made based on the consideration that the target learners might be from different linguistic backgrounds and non-native English speakers might have different proficiency levels of English (see Table 5.5). Specifically, the loan words were separated from other English words because they might pose more challenges to learners who do not have knowledge of the Chinese language. 
Table 5.5: Three levels of each sub-list in the TCM technical word list

\begin{tabular}{|c|c|c|c|}
\hline Level & Level 1 & Level 2 & Level 3 \\
\hline Sub-list 1 & $\begin{array}{l}\text { The most frequent } \\
\text { 1,000 word types } \\
\text { which are from 1,000- } \\
\text { 3,000 BNC/COCA word } \\
\text { families }\end{array}$ & $\begin{array}{l}\text { The most frequent } \\
\text { 1,000 word types } \\
\text { which are outside } \\
\text { 1,000-3,000 } \\
\text { BNC/COCA word } \\
\text { families }\end{array}$ & $\begin{array}{l}\text { The most frequent } \\
1,000 \text { word types } \\
\text { which are loan words }\end{array}$ \\
\hline Sub-list 2 & $\begin{array}{l}\text { The most frequent } \\
2,000 \text { word types } \\
\text { which are from 1,000- } \\
3,000 \text { BNC/COCA word } \\
\text { families }\end{array}$ & $\begin{array}{l}\text { The most frequent } \\
2,000 \text { word types } \\
\text { which are outside } \\
\text { 1,000-3,000 } \\
\text { BNC/COCA word } \\
\text { families }\end{array}$ & $\begin{array}{l}\text { The most frequent } \\
2,000 \text { word types } \\
\text { which are loan words }\end{array}$ \\
\hline Sub-list 3 & $\begin{array}{l}\text { Outside the most } \\
\text { frequent 2,000 word } \\
\text { types but from 1,000- } \\
3,000 \text { BNC/COCA word } \\
\text { families }\end{array}$ & $\begin{array}{l}\text { Outside the most } \\
\text { frequent 2,000 word } \\
\text { types and outside } \\
\text { 1,000-3,000 } \\
\text { BNC/COCA word } \\
\text { families }\end{array}$ & $\begin{array}{l}\text { Outside the most } \\
\text { frequent 2,000 word } \\
\text { types which are loan } \\
\text { words }\end{array}$ \\
\hline
\end{tabular}

Technical words from the fourth 1,000 word families of BNC/COCA lists onwards were separated from that from the first to the third 1,000. This decision was made because it is unlikely that the undergraduates have mastered the vocabulary beyond the third 1,000 BNC/COCA levels in the EFL contexts such as Vietnam (Dang, 2017; Nguyen \& Webb, 2017), Indonesia (Nurweni \& Read, 1999), Denmark (Henriksen \& Danelund, 2015), Israel (Laufer, 1998), and Taiwan (Webb \& Chang, 2012). Thus, there is a reason to expect that a considerable number of the TCM learners from the EFL contexts such as China find technical words which are outside the high-frequency vocabulary band equally difficult.

To make sure that the TCM technical word list benefits learners from different linguistic backgrounds and at different vocabulary levels, the characteristics of this list have been 
established. The next subsection describes the criteria of selecting items for the TCM technical word list based on the abovementioned characteristics.

\subsubsection{Setting criteria for the items in the TCM technical word list}

Based on the discussion of characteristics of the TCM technical word lists, a selected item had to satisfy the meaning, specialized occurrence and frequency criteria. However, the aforementioned technical words had satisfied the specialized occurrence and specialized meaning criteria during the process of being identified as technical (see Section 4.4 and Section 5.2 for details). Therefore, this subsection is mainly devoted to describing the establishment of the frequency criterion for the items to be included in the TCM technical word lists.

Frequency is the commonly used criterion in deciding whether a word is worthy of being included in a word list (Coxhead, 2000; Gardner \& Davies, 2014; Wang et al., 2008; Yang, 2015). There is no unanimous frequency threshold that can be applied to every corpusbased word list study. The frequency threshold varied from study to study depending on the aim of the word list. For example, Coxhead (2000) used the frequency criterion of at least 100 times to select the AWL word families. That is, a selected word family had to occur at least 100 times in a 3.5-million academic written corpus. This threshold was later adopted by a considerable number of specialized word list studies (e.g., Khani \& Tazik, 2013; Mudraya, 2006; Valipouri \& Nassaji, 2013; Wang et al., 2008; Yang, 2015). Using word type as the unit of counting, Ha (2015) adopted a minimum of 63 occurrences in 6.7-million financial corpora to select technical words in finance. This threshold equals to 100 occurrences in Coxhead's (2000) word-family-based frequency threshold, taking the average number of each word family into account.

In this study, the frequency cut-off point of 60 times was chosen after comparing five pilot lists as shown in Table 5.6. Adopting the same frequency criterion of Coxhead (2000) and Ha (2015) means that the selected TCM technical word types had to occur at least 33 times in the 3.5-million TCM Corpora. This frequency criterion led to a pilot list with 3,695 word types (see Table 5.6). Such a long listing of lexical items might be too cumbersome to be a realistic and attainable learning goal. Therefore, four other cut-off points above 33 times were trialed as shown in Table 5.6. The comparison showed that, 
of the five frequency cut-off points, 60 times led to a much shorter listing of word types without undermining the coverage too much. It was thus decided that 60 times is the cutoff point for the TCM technical word list.

Table 5.6: Comparison of coverage and number of items in five pilot lists with 5 frequency cut-off points

\begin{tabular}{|l|l|l|}
\hline Frequency cut-off points & Number of word types & Coverage \\
\hline 33 & 3,695 & $37.84 \%$ \\
\hline 40 & 3,407 & $37.54 \%$ \\
\hline 50 & 3,069 & $37.11 \%$ \\
\hline 55 & 2,910 & $36.87 \%$ \\
\hline $\mathbf{6 0}$ & $\mathbf{2 , 7 7 8}$ & $\mathbf{3 6 . 6 4 \%}$ \\
\hline
\end{tabular}

Range and dispersion were widely used in the studies of specialized word list (e.g., Coxhead, 2000; Gardner \& Davies, 2014; Lei \& Liu, 2016; Wang et al., 2008; Yang, 2015) but not in the present study. This was determined by the nature of the TCM technical word list, which is a technical word list. Technical vocabulary may only appear frequently in a single text or chapter, reflecting the specific topic and content of that text or chapter (Miller \& Biber, 2015). This nature of technical vocabulary means that it is highly likely that technical words do not occur in a wide range of texts or distribute widely across the target corpus. This idea was confirmed by Fraser (2007), who found that parasympathetic was a technical word in pharmacology that should be known to all pharmacology learners but found in only three articles. In order to ensure that such technical words are included in the word list, it was decided to build a technical word list without range and dispersion criteria.

In sum, the comparison of five pilot lists with different frequency cut-off points showed that 60 resulted in a word list that had a smaller number of items but still provided a high coverage over the TCM Corpora.

\subsubsection{Selecting items for and validating the TCM technical word list}

The previous section has described the establishment of the meaning, specialized occurrence and frequency criteria of the TCM technical word list based on the 
characteristics discussed in Section 5.3.1. The lexical items that satisfied the criteria were selected and those items were divided into three sub-lists. Each sub-list was then further divided into three categories as mentioned in Table 5.5.

To validate the TCM technical word list, a validating TCM corpus was developed. In principle, the structure of the validating corpus should mirror the structure of the TCM Corpora, from which the word list was developed. However, due to the availability of materials, the validating corpus was relatively small. It consisted of one TCM textbook (54,757 tokens) and 120 journal articles (409,995 tokens) from the four journals mentioned in Section 4.2.3. The journal articles were also sampled in a similar way to that of the TCM journal article corpus (see Section 4.2.3): 40 articles each from Chinese herbal medicine, alternative therapies, and basic research into theories, diagnoses and other general aspects of TCM. In total, the validating TCM corpus is comprised 464,752 tokens. The coverage of the TCM technical word list over the validating TCM corpus was determined by submitting this corpus to the Range program (Heatley et al., 2002).

For the same purpose, the coverage of the TCM technical word list over the Western Medical Corpora (WM Corpora) and the general written English corpus were also calculated. The details of the WM Corpora can be found in Section 4.3, while the components of the general written English corpus can be found in Section 5.2.2, which acted as a comparison corpus to identify technical words.

\subsubsection{Specifications for the TCM technical word list}

To provide the potential users of the TCM technical word list with a clear view of the TCM technical word list, this section adopts and adapts Nation's (2016, p.133-134) specification framework to describe this list as a way to summarize the characteristics of this list. Table 5.7 presents the detailed specifications of the TCM technical word list, focusing on 13 different aspects.

In the next section, the methodology related to the identification of technical lexical bundles in the TCM texts and the development of the TCM technical lexical bundle list will be described. 
Table 5.7: Specifications for the TCM technical word list

\begin{tabular}{|c|c|}
\hline Focus & Specification \\
\hline Purpose & $\begin{array}{l}\text { Vocabulary learning targets for TCM learners } \\
\text { Course design and material writing for TCM practitioners }\end{array}$ \\
\hline Size of list & 2,778 items \\
\hline Unit of counting & Word types \\
\hline Number of sub-lists & $\begin{array}{l}\text { Nine sub-lists to adapt to learners' linguistic background and } \\
\text { vocabulary levels. }\end{array}$ \\
\hline Capitalization & No distinction between non-capitalized and capitalized words \\
\hline Numbers & Some technical words contain numbers such as $\mathrm{BCl} 2$ \\
\hline Criteria for list & $\begin{array}{l}\text { (1) Keyness: has to be more frequent in the TCM Corpora over } \\
\text { general English corpus; } \\
\text { (2) Meaning: has a meaning related to the subject knowledge; } \\
\text { (3) Frequency: occurs more than } 60 \text { times in the TCM Corpora. }\end{array}$ \\
\hline Word separators & Space, hyphens \\
\hline Separate lists & $\begin{array}{l}\text { Proper nouns, marginal words, transparent compounds, and } \\
\text { abbreviations are in four separate lists as specified in Chapter } \\
4 \text {. }\end{array}$ \\
\hline Function words & Not included in this list. \\
\hline Homoforms & Homoforms and homographs are not distinguished. \\
\hline Corpus & $\begin{array}{l}\text { The TCM Corpora consists of a theory-based textbook corpus, } \\
\text { a practice-based textbook corpus, and a journal article corpus, } \\
\text { which contains } 3,478,267 \text { tokens in total. }\end{array}$ \\
\hline Possible criticisms & $\begin{array}{l}\text { (1). Homoforms and homographs in the list are not } \\
\text { distinguished (e.g., shen means kidney vs shen means spirit); } \\
\text { (2). The TCM Corpora on which this list was developed are } \\
\text { relative small in size; } \\
\text { (3) The TCM Corpora only involves written materials. }\end{array}$ \\
\hline
\end{tabular}




\subsection{Developing the TCM technical lexical bundle list}

A mixed-method approach of corpus-based quantitative analysis using a computer program and qualitative semantic analysis was used to identify the most frequently used technical lexical bundles in TCM. The corpus-based computational analysis was to generate a provisional lexical bundle list, whereas the qualitative analysis was to decide whether a lexical bundle in the provisional list is technical or not. The remainder of this section starts with deciding the length of lexical bundles and word unit of analysis (Section 5.4.1) to make preparation for computational analysis, followed by a description of computational analysis (Section 5.4.2) and criteria for qualitative analysis (Section 5.4.3). To make the technical lexical bundle list more concise, the last subsection describes the process of how the lexical bundle list is refined.

\subsubsection{Deciding the length of lexical bundles and word unit of analysis}

Before proceeding to the data-driven computational analysis, it is important to decide the length of lexical bundles to be investigated and the word unit of analysis. This study adopted the term 'lexical bundles' because it aims to look at the multiword sequences that occur most frequently in the discourse community of TCM, following the approach of Biber and Barbieri (2007, p. 264). However, this study extended the original use of lexical bundles from three or more words (Biber et al., 1999) to two to five words even though it is argued that 2-word lexical bundles are usually subsumed in 3-, 4-, and 5-word bundles (Simpson-Vlach \& Ellis, 2010). The reason for this is that some of the important technical lexical bundles in TCM, such as the names of herbal medicine, are in two-word combination (e.g., Gan Cao, Fu Ling). To make sure that these bundles were included, this study decided to include 2-word bundles. In terms of the unit of analysis, word type was adopted in order to be consistent with the single word analysis. This means blood vessel and blood vessels were regarded as two different lexical bundles.

\subsubsection{Extracting the lexical bundles}

After deciding the length of the lexical bundles to be included and word unit of analysis, the next step is to extract the lexical bundles. Using the same TCM Corpora (see Section 4.2 for details), the function of WordList Cluster in Wordsmith (Scott, 2012) was performed to retrieve a provisional lexical bundle list. In total, this data-driven lexical bundle list contained over 220,000 entries with the most frequent 20 lexical bundles presented in Table 5.8. This means that the provisional lexical bundle list is a long list of 
lexical bundles which required substantial amount of further work. Thus, it is important to set the frequency criterion to make the lexical bundle list into more manageable size. An initial look at the bundles in Table 5.8 shows that a large number of the top bundles in the TCM Corpora were made up of high-frequency function words, and were nontechnical in nature (e.g., of the, in the, to the). Such lexical bundles were called 'grammatical collocations' by Durrant (2009, p. 163).

Table 5.8: The most frequent 20 lexical bundles in the TCM Corpora

\begin{tabular}{|l|l|l|l|}
\hline 1 & of the & 11 & is the \\
\hline 2 & in the & 12 & qi and \\
\hline 3 & to the & 13 & is a \\
\hline 4 & and the & 14 & Chinese medicine \\
\hline 5 & on the & 15 & there is \\
\hline 6 & it is & 16 & at the \\
\hline 7 & from the & 17 & can be \\
\hline 8 & with the & 18 & that the \\
\hline 9 & such as & 19 & the heart \\
\hline 10 & for the & 20 & due to \\
\hline
\end{tabular}

Another characteristic of the provisional lexical bundle list is that, as presented in Table 5.8, a certain number of high frequency lexical bundles (e.g., qi and, if liver) are of little pedagogical benefit since they do not create any extra learning burden if the learners know the keywords qi and liver respectively. Those features of the bundles in the provisional lexical bundle list highlight a need to set meaning criterion to make the lexical bundle list more pedagogically useful. In the next subsections, the frequency and meaning criteria for selecting technical lexical bundles will be described in turn.

\subsubsection{Deciding frequency criterion for TCM technical bundle list}

In previous studies, the frequency threshold for lexical bundles varied from five times per million words (Ackermann \& Chen, 2013), ten times per million words (Simpson-Vlach \& Ellis, 2010), 20 times per million/words (Byrd \& Coxhead, 2010; Cortes, 2004; Ha, 2015; Hyland, 2008; Liu, 2012), to 25 times per million words (Wood \& Appel, 2014) depending on the specific research aims. Since the aim of the present research is to better 
understand the nature of technical vocabulary in TCM, a less restricted cut-off point of 20 instances per million words was adopted. This means the cut-off frequency threshold for this study was set at 70 times in the 3.5-million-word TCM Corpora. Lexical bundles with occurrences under 70 were thus not included in the following semantic-related analysis. This step decreased the provisional lexical bundle list to 8,164 entries.

\subsubsection{Deciding meaning criterion for TCM technical bundle list}

In addition to the frequency criterion mentioned above, another two meaning-related selection criteria were used: whether the meaning of each of the lexical bundles is complete and whether the meaning of the lexical bundles relates to the subject knowledge of TCM. The analysis for the completeness of meaning was carried out using concordance lines. For instance, Radix Paeoniae is part of the name of an herb Radix Paeoniae Lactiflorae; nonetheless, it is not easy to decide whether Radix Paeoniae is meaning complete unless the concordance lines were consulted. In this way, a more pedagogically valuable list of lexical bundles can be created to help improve the acquisition of technical vocabulary in the discipline of TCM.

The second meaning-related criteria is that the meaning of a lexical bundle should be related to subject knowledge of TCM to be considered as a technical lexical bundle. The identification of lexical technical bundles is easier compared to identification of the technical words since they can be signaled by the presence of technical words - either one of the constituents is a technical word (e.g., side effects, on the back, large intestine, to resolve phlegm), or all of the constituents are technical words (e.g., Bai Zhu, Yin deficiency, liver blood, Cinnamomi Cassiae).

In sum, the criteria used for selecting technical lexical bundles are: (1) frequency >= 70; (2) meaning complete; and (3) meaning related to TCM subject knowledge. Every entry which satisfied the frequency criterion was checked, and those which satisfied the meaning-based criteria mentioned above were selected. Once this process was completed, a list of the most distinctively technical lexical bundles was created, comprising 1,137 items. 


\subsubsection{Refining the TCM technical lexical bundle list}

There is considerable overlap among the 1,137 technical lexical bundles, for example, the ethereal soul and ethereal soul. In other words, considerable numbers of two-, three-, and four-word lexical bundles are subsumed in the three-, four-, and five-word bundles. While this is a problem that has been identified by other researchers (Biber \& Barbieri, 2007; Byrd \& Coxhead, 2010; Hyland, 2008), only Wood and Appel's (2014) study to date has dealt with this problem. Wood and Appel (2014, p. 5) advanced this field of research by listing the root structure of lexical bundles with variable slots at the end of the sequences being placed in brackets. First, they decided that if a shorter lexical bundle is subsumed in another longer lexical bundle, the shorter lexical bundle is considered as the root structure. For example, the three-word structure 'as long as' was found to be part of the four-word structure 'as long as the'. As a result, 'as long as' was classified as the root structure with 'the' being the variable slot, and the whole structure was presented as 'as long as (the)'. Second, they also decided that wherever two 4-word bundles contained an identical 3-word structure, the 3-word string common to both sequences was classified as the root structure. For example, the four-word sequences 'at the end of' and 'the end of the' were combined to create the structure '(at) the end of (the)'.

In order to deal with the overlapping lexical bundles, an additional step was implemented in the present study following Wood and Appel's (2014) approach of identifying root structure of lexical bundles. The general principle is if a shorter lexical bundle is subsumed in another or other longer lexical bundle(s), the shorter or shortest lexical bundle is considered as the root structure. Here are some instances. 'Blood glucose' is embedded in 'blood glucose levels', 'blood glucose' is thus the root structure and 'levels' is the variable slot which would be placed brackets in the end of 'blood glucose' as 'blood glucose (levels)'. If there are two or more options at the periphery of a structure, all options would be bracketed as in the following case. 'Liver blood' is subsumed in 'nourish liver blood' 'deficiency of liver blood' 'nourishes liver blood', 'liver blood' is thus considered to be the root structure in this case. The other variable slots 'nourish' 'deficiency of and 'nourishes' were placed in brackets in front of 'liver blood' as '(nourish/deficiency of/nourishes) liver blood'. 
By condensing the overlapping lexical bundles, the number of technical lexical bundles was reduced, especially lexical bundles that start with the/a or ended in of. This step makes the lexical bundle list a more pedagogically efficient and friendly list with 898 items. The results will be presented in Section 5.5.7.

\subsection{Results}

This section sets out to report on the results and findings in response to the research questions raised in Section 5.1 of the present study.

\subsubsection{TCM technical words from 1 to $25 \mathrm{BNC} / \mathrm{COCA}$ word lists}

In answer to the first research question of which lexical items in Nation's (2012) 1-25 frequency-based BNC/COCA word lists are technical words, a total number of 4,368 word types were identified as technical. Altogether, these words covered $33.50 \%$ of the total tokens in the TCM Corpora. Table 5.9 presents the distributional profile of these technical words over Schmitt and Schmitt's (2014) high, mid, and low-frequency vocabulary.

Table 5.9: Distribution of BASEWRD 1-25 technical words across high, mid, and low-frequency vocabulary bands

\begin{tabular}{|l|l|l|}
\hline Frequency band & No. of types & Proportion \\
\hline High-frequency $(1,000-3,000)$ & 1,757 & $40.22 \%$ \\
\hline Mid-frequency $(4,000-8,000)$ & 1,221 & $27.95 \%$ \\
\hline Low-frequency $(9,000-25,000)$ & 1,390 & $31.83 \%$ \\
\hline Total & 4,368 & $100 \%$ \\
\hline
\end{tabular}

As shown in Table 5.9, of these 4,368 word types, 1,757 items of the technical words were found in the first to third 1,000 word families (high-frequency vocabulary) based on Nation's BNC/COCA word lists. They accounted for $40.22 \%$ of the word types identified from 1-25 BNC/COCA base word lists, which means high-frequency vocabulary played an important role in lexicalizing the technical sense of the TCM texts. These results means that a good number of the technical words (e.g., heart, wind) might be familiar to beginners/laypersons. Table 5.10 illustrates the most frequent 50 technical words which were identified from BASEWRD 1 to 25 with the bold items from the high-frequency vocabulary band, the underlined items from the mid-frequency vocabulary band, and the others from the low-frequency vocabulary band. 
Table 5.10: Top 50 TCM technical words from Nation's (2006) BNC/COCA word lists

\begin{tabular}{|c|c|c|c|c|c|}
\hline & Word type & $\begin{array}{ll}\text { No. } & \text { of } \\
\text { occurrences } & \\
\end{array}$ & & Word type & $\begin{array}{ll}\text { No. } & \text { of } \\
\text { occurrence } & \\
\end{array}$ \\
\hline 1 & blood & 13,925 & 26 & clinical & 4,707 \\
\hline 2 & treatment & 13,433 & 27 & pulse & 4,656 \\
\hline 3 & heat & 12,039 & 28 & channel & 4,646 \\
\hline 4 & yin & 10,453 & 29 & decoction & 4,079 \\
\hline 5 & pain & 10,252 & 30 & lower & 4,004 \\
\hline 6 & $\underline{\text { liver }}$ & 9,976 & 31 & damp & 3,977 \\
\hline 7 & deficiency & 8,956 & 32 & $\underline{\text { lung }}$ & 3,903 \\
\hline 8 & acupuncture & 8,484 & 33 & formula & 3,876 \\
\hline 9 & used & 7,749 & 34 & use & 3,703 \\
\hline 10 & patients & 7,627 & 35 & effect & 3,664 \\
\hline 11 & group & 7,311 & 36 & patient & 3,648 \\
\hline 12 & kidney & 6,946 & 37 & disease & 3,638 \\
\hline 13 & spleen & 6,878 & 38 & dampness & 3,562 \\
\hline 14 & heart & 6,760 & 39 & fire & 3,432 \\
\hline 15 & cold & 6,415 & 40 & $\underline{\text { herbs }}$ & 3,372 \\
\hline 16 & medicine & 6,276 & 41 & syndrome & 3,268 \\
\hline 17 & points & 6,229 & 42 & $\underline{\operatorname{tang}}$ & 3,262 \\
\hline 18 & point & 5,964 & 43 & results & 3,251 \\
\hline 19 & symptoms & 5,693 & 44 & pattern & 3,241 \\
\hline 20 & study & 5,526 & 45 & control & 3,183 \\
\hline 21 & phlegm & 5,482 & 46 & cases & 3,013 \\
\hline 22 & body & 5,406 & 47 & chronic & 2,969 \\
\hline 23 & wind & 5,340 & 48 & channels & 2,959 \\
\hline 24 & tongue & 5,323 & 49 & effects & 2,945 \\
\hline 25 & stomach & 5,038 & 50 & groups & 2,893 \\
\hline
\end{tabular}

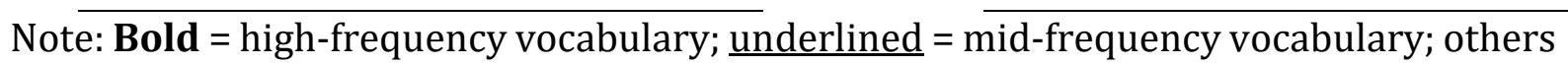
= Low-frequency vocabulary. 
As shown in Table 5.10, high-frequency words such as blood, treatment, and heat have rather high occurrences, and are essential in conveying core technical meaning in TCM texts. Comparatively speaking, slightly fewer technical words were from the midfrequency vocabulary band (1,221 word types) than the high-frequency bands as seen in Table 5.9. Technical words from the mid-frequency band such as liver, deficiency, and kidney (see Table 5.10) are also of fundamental importance because they represent some of the essential concepts in TCM theory. An interesting word found at the mid-frequency level is tang. It means "a taste or smell that is strong and bitter in a pleasant way" according to Macmillan dictionary (2009-2017). However, in TCM, tang is a loan word from Chinese which means decoction. Though this kind of semiotic coincidence in English and Romanized Chinese is not very common, it definitely creates confusion for learners.

Similarly, a considerable number of technical words were from the low-frequency vocabulary band (1,390 word types). It is not surprising that more word types were identified from the low-frequency band than the mid-frequency band due to the specificity of vocabulary in technical areas. These kinds of words, such as acupuncture, spleen and decoction (see Table 5.10), are very unlikely to be met in general English. It is interesting to note that loan word yin was identified in the frequency-based BNC/COCA lists since most loan words are outside the frequency-based lists and have been grouped into the TCM loan word list. According to the Merriam-Webster Dictionary, yin was defined as "the feminine passive principle in nature that in Chinese cosmology is exhibited in darkness, cold, or wetness and that combines with yang to produce all that comes to be" (yin, n.d.). There is no other homograph of yin defined in the dictionary in the English language. It might be because yin has become a well-known concept in English since it is also an important concept in Chinese philosophy. The concordances were also drawn from the COCA to illustrate the use of yin, which supported that yin is a loan word from Chinese as can be seen from the following examples.

1. He gets involved with a guru who claims that homosexuality creates an imbalance of yin and yang in the world.

2. The cold ice cream and hot rum sauce signify the complementary energies of yin and yang, female and male. 
It is interesting that yang was not found in the 1-25 frequency-based BNC/COCA word lists but in the proper noun list. In Chinese culture, yin and yang always come together as two opposite sides or parts of something. The reason for this may be that Yang is also a common family name in Chinese. This linguistic phenomenon is explained in the limitation section (see Section 8.4.2 for details).

Overall, the results show that TCM technical words cut across the high, mid, and lowfrequency vocabulary bands of the BNC/COCA word lists, which conforms to that of Nation et al. (2016). Further, those technical words were spread across the high, mid, and low-frequency vocabulary bands with a similar proportion. In the following section, the semantic features of those technical words will be further explored and reported.

\subsubsection{The semantic features of BASEWRD 1-25 technical words in the TCM texts}

This section answers the question about the semantic features of the technical words from the frequency-based BNC/COCA word lists. The technical words from the first to the $25^{\text {th }}$ BNC/COCA based word lists fall into four groups based on their semantic usage patterns as shown in Table 5.11.

Table 5.11: Semantic features of technical words from BASEWRD 1-25 of BNC/COCA lists in TCM

\begin{tabular}{|l|l|l|}
\hline Semantic feature & Description & Examples \\
\hline $\begin{array}{l}\text { General } \\
\text { technical words }\end{array}$ & $\begin{array}{l}\text { those words are used in much the } \\
\text { same way in both general language } \\
\text { and TCM }\end{array}$ & patient, eye, pain \\
\hline $\begin{array}{l}\text { Specialized } \\
\text { technical words }\end{array}$ & $\begin{array}{l}\text { those words are used with an } \\
\text { additional technical meaning in TCM }\end{array}$ & cold, stroke, labor \\
\hline Metaphorical & $\begin{array}{l}\text { those words are used with an } \\
\text { extension of the general meaning }\end{array}$ & bone, heart, wind \\
\hline $\begin{array}{l}\text { Highly } \\
\text { technical words }\end{array}$ & $\begin{array}{l}\text { those words have constant meaning } \\
\text { and are only likely to be met in TCM } \\
\text { or its related disciplines }\end{array}$ & $\begin{array}{l}\text { chromosome, } \\
\text { decoction }\end{array}$ \\
\hline
\end{tabular}


The first group of the technical words in TCM is that those words were used in the same way in a technical field as in general English texts, while the second group is that some general words could take on specific technical meanings which were different from their general meanings. The third one is that the technical words could be used metaphorically in TCM to lexicalize the technical knowledge. One kind of metaphorical use identified is, for example, bone. Bone is not the same concept in TCM as in general medical science. As defined by Maciocia (2005, p. 83), "bones in Chinese medicine does not purely refer to the Western anatomical structure of that name, although it does include that. Apart from its anatomical aspect, bones in Chinese medicine also refer to an energetic layer of the body structures." This definition clearly shows that bone in TCM is conceptually richer than what is defined in medical science in that the meaning of bones has been semantically transferred to denote energetic layer.

Another kind of metaphorical use is exemplified by word such as wind. Wind in TCM is more than the climatic wind, it is also one cause of diseases; however, in Western medical science there is no such equivalent concept about wind being a disease factor. This is because TCM was developed based on the observation of life processes and the relationships between human beings and their environment (Kaptchuk, 2001). This is a method of description that is not available in Western medicine which relies largely on the mechanism of biomedicine. Understanding wind properly in TCM, therefore, involves some conceptual thinking in this specialized discipline, taking into account the underlying cultural traditions and philosophies. Similar cases are fire, heat and others which have no equivalent concept in Western Medicine. As shown in the fifth row of Table 5.11, the fourth group of technical words from BASEWRD 1-25 is that some highly technical words (e,g., acupuncture) have constant meaning and have only one denotation (Hsu, 2013). Usually, those words are only likely to be met in TCM or its related disciplines such as general medicine.

Taken together, the analysis of semantic features of technical words from BASEWRD 125 further illustrates the lexical complexity of and challenges posed by such TCM lexis. It indicates that the semantic features of a word are also important indicators for selecting entries for discipline-specific word lists. 


\subsubsection{How large is the technical vocabulary of TCM?}

This subsection answers the question about the overall coverage of technical vocabulary in the TCM texts. Table 5.12 shows the coverage of technical words identified from the frequency- and range-based BNC/COCA word lists was obtained (BASEWRD 1-25 technical words), and that of technical words outside the BNC/COCA word lists (BASEWRD 35 and 36) identified in the first study.

Table 5.12: Summary of technical words in TCM

\begin{tabular}{|l|l|l|}
\hline Word list & Word types & Coverage \\
\hline BASEWRD 1-25 technical words & 4,368 & $33.51 \%$ \\
\hline BASEWRD 35 TCM loan words & 1,261 & $3.29 \%$ \\
\hline BASEWRD 36 TCM words & 6,806 & $2.66 \%$ \\
\hline Total & 12,435 & $39.46 \%$ \\
\hline
\end{tabular}

As presented in Table 5.12, a total number of 12,435 word types were identified as technical words, and they covered $39.46 \%$ of the total tokens in the whole TCM Corpora. This means that almost four out of ten running words in the English-medium TCM texts are technical words. This percentage is higher than $31.2 \%$ found by Chung and Nation (2004) in anatomy texts. Such a high density of technical vocabulary highlights the importance of technical vocabulary in this discourse community.

\subsubsection{The most frequent technical words in TCM}

The third research question of the present study asks which technical words are frequently used in TCM so that they can be included in the TCM technical word list. In total, 2,778 word types satisfied frequency and meaning criteria mentioned in Section 5.3.2, and were included in the TCM technical word list. Table 5.13 presents the most frequent 20 technical words from this list with their total number of occurrences in the TCM Corpora provided. The loan words (from TCM-specific loan based word list) are in bold to make them more visible in the list. As can be seen, these technical words occurred very frequently in the TCM texts and are thus essential to the learners. 
Table 5.13: The most frequent 20 items in the TCM technical word list

\begin{tabular}{|l|l|l|l|}
\hline Technical word & No. of occurrences & Technical word & No. of occurrences \\
\hline qi & 21,866 & used & 7,749 \\
\hline blood & 13,924 & patients & 7,627 \\
\hline treatment & 13,433 & group & 7,311 \\
\hline heat & 12,039 & radix & 7,294 \\
\hline yin & 10,453 & kidney & 6,945 \\
\hline pain & 10,252 & spleen & 6,878 \\
\hline liver & 9,976 & heart & 6,760 \\
\hline yang & 9,800 & cold & 6,415 \\
\hline deficiency & 8,956 & medicine & 6,276 \\
\hline acupuncture & 8,484 & points & 6,229 \\
\hline
\end{tabular}

The 2,778-item TCM technical word list was divided into three sub-lists, with sub-list 1 containing the most frequent 1,000 technical words identified in the TCM Corpora, sublist 2 containing the second most frequent 1,000 technical words, and sub-list 3 containing the third most frequent 778 technical words. Table 5.14 summarizes the coverage figures of each sub-list with examples provided.

Table 5.14: Coverage of the TCM technical word list

\begin{tabular}{llll}
\hline Sub-list & Word types & Coverage & Examples \\
\hline 1 & 1,000 & $29.97 \%$ & qi, blood, treatment \\
\hline 2 & 1,000 & $4.89 \%$ & detected, laboratory, geng \\
\hline 3 & 778 & $1.78 \%$ & ultrasound, vein, fuling \\
\hline Total & 2,778 & $36.64 \%$ & \\
\hline
\end{tabular}

The TCM technical word list covered $36.64 \%$ of the total tokens in the TCM Corpora. Most importantly, the first 1,000 words in the TCM technical word list covered $29.97 \%$ of the running words, which was much higher than the second and third sub-lists. Each sub-list was further divided into three levels. Level 1 and 2 contain technical words from the first 1,000 to the third 1,000 BNC/COCA word families, and those outside the most frequent 3,000 word families. Level 3 are items borrowed from Chinese, namely loan words. Table 
5.15 presents the number of word types, coverage and examples of technical words in each level of sub-lists. In total, there are 1,325 word types in the TCM technical word list from Level 1 (the most frequent 3,000 BNC/COCA word families), making up 22.21\% of the TCM texts. A similar number of 1,245 word types of the TCM technical word lists are at Level 2 (outside the most frequent 3,000 BNC/COCA word families), covering $11.46 \%$ of the TCM Corpora. A much smaller number of 218 word types are loan words at Level 3 , covering a much less but important $2.97 \%$ of the TCM texts.

Table 5.15: Number of word types and coverage of technical words at each level of sub-lists

\begin{tabular}{|c|c|c|c|c|c|c|}
\hline \multirow[t]{2}{*}{ Level } & \multicolumn{2}{|c|}{ Level 1} & \multicolumn{2}{|c|}{ Level 2} & \multicolumn{2}{|c|}{ Level 3} \\
\hline & word types & coverage & word types & coverage & word types & coverage \\
\hline Sub-list 1 & 643 & $19.39 \%$ & 289 & $8.13 \%$ & 68 & $2.45 \%$ \\
\hline Sub-list 2 & 465 & $2.33 \%$ & 468 & $2.23 \%$ & 67 & $0.33 \%$ \\
\hline Sub-list 3 & 217 & $0.49 \%$ & 478 & $1.1 \%$ & 83 & $0.19 \%$ \\
\hline Total & 1,325 & $22.21 \%$ & 1,235 & $11.46 \%$ & 218 & $2.97 \%$ \\
\hline
\end{tabular}

As can also be seen from Table 5.15, the division of the TCM technical word list into levels in each sub-list has made the 2,778-item technical word list into 9 smaller sets of technical words with the largest set having 643 items. Such division has two benefits. First, it makes the TCM technical word list adaptable to learners from different backgrounds and at different vocabulary levels (see Section 7.4.4 for details). Second, it condenses the long TCM technical word list into small and manageable sets of technical words. The TCM technical words in each sub-list and level can be seen in Appendices 2 to 11.

\subsubsection{Coverage of the TCM technical word list in TCM theory and practice-based textbooks, and journal articles}

The fourth research question concerns the coverage of the TCM technical word list in TCM theory and practice-based textbooks, and journal articles. As demonstrated in Table 5.16 , the TCM technical word list provided a maximum coverage $(40.06 \%)$ over the TCM practice-based textbooks, which was slightly higher than that of the theory-based textbooks (38.54\%). The coverage of the TCM technical word list over the journal articles 
was considerably lower than that of the textbooks in general, at $31.43 \%$. These results are not surprising since they are consistent with the findings of Study 1 , which found that journal articles were less lexically demanding than textbooks as journal articles used relatively more general high-frequency vocabulary.

Table 5.16: Coverage of the TCM technical word list in different types of TCM texts

\begin{tabular}{|l|l|}
\hline Text types & Coverage (\%) \\
\hline Theory-based textbooks & $38.54 \%$ \\
\hline Practice-based textbooks & $40.06 \%$ \\
\hline Journal articles & $31.43 \%$ \\
\hline
\end{tabular}

Despite these differences, the coverage of the TCM technical word list in the three types of TCM texts were generally at a high level of over 30\%. This means the TCM learners can encounter those items in the TCM technical word list frequently in both TCM textbooks and journal articles.

\subsubsection{Coverage of the TCM technical word list in an independent collection of TCM texts, Western Medical texts, and general written English texts}

The fifth research question concerns the coverage of the TCM technical word list in another collection of TCM texts, in Western Medical (WM) texts, and in general written English texts with an aim to validate the TCM technical word list. Table 5.17 presents the coverage figures of the TCM technical word lists over those three different collection of texts.

Table 5.17: Coverage of the TCM technical word list in three validating corpora

\begin{tabular}{|l|l|}
\hline Corpus & Coverage \\
\hline TCM validating corpus & $31.43 \%$ \\
\hline WM Corpora & $25.32 \%$ \\
\hline General written English corpora & $4.11 \%$ \\
\hline
\end{tabular}

The second row of Table 5.17 shows that the TCM technical word list covered $31.43 \%$ of the TCM validating corpus (see Section 5.3.3). This coverage figure is interesting as it is exactly the same as the coverage of the TCM journal articles mentioned in Table 5.16 
above. This might be because the TCM validating corpus is, to a large extent, a journal article corpus due to a lack of textbooks. This finding well demonstrates that the TCM technical word list can reflect the frequently used technical words in the discipline of TCM.

In contrast, the TCM technical word list only accounted for $4.11 \%$ of the general written English texts as shown in the last row of Table 5.17. Such coverage was mainly achieved by items such as arm, brain, and chest. This coverage figure means that the TCM technical word list represents a group of technical words that can be rarely encountered in general English language use. Moreover, the TCM technical word list covered a considerably lower proportion of the Western Medical texts (25.32\%) than that of the TCM Corpora and TCM validating corpus. This coverage was achieved by items such as antibiotics, cell, and diagnoses. The result suggests that the TCM technical word list better represents the most frequent technical words in TCM rather than Western Medicine.

Taken together, these findings indicate that the TCM technical word list can accurately capture the most frequent technical words in the discipline of TCM. In the next subsection, the results related to the technical lexical bundles will be reported.

\subsubsection{The most frequent technical lexical bundles in TCM}

The last research question of the present study is about the most frequent technical lexical bundles in the TCM Corpora. In total, 1,137 lexical bundles satisfied the included criteria described in Section 5.4.3 and 5.4.4. To deal with the overlapping lexical bundles (see Section 5.4 .5 for more details), these 1,137 items were refined and resulted in a TCM technical bundles list of 898 items. Table 5.18 presents the most frequently used 20 lexical bundles after refinement, which were arranged according to their number of occurrences in the TCM Corpora. The root structure of each lexical bundle is in bold to make them more visible. As can be seen, those lexical bundles occurred quite frequently in the TCM Corpora. 
Table 5.18: The most frequent 20 condensed lexical bundles in the TCM Corpora

\begin{tabular}{|c|c|}
\hline Lexical bundles & No. of occurrences \\
\hline 1. (traditional) Chinese medicine (practitioners) & 3,369 \\
\hline 2. (resolves/the/resolve) damp heat & 1,862 \\
\hline 3. (the) yin deficiency & 1,858 \\
\hline $\begin{array}{l}\text { 4. (spleen and/tonify/tonifies/deficiency of/the/warm) } \\
\text { kidney yang }\end{array}$ & 1,732 \\
\hline $\begin{array}{l}\text { 5. (stagnation of/flow of/move/moves/the/stagnant/ } \\
\text { rebellious/free flow of) liver qi }\end{array}$ & 1,644 \\
\hline 6. (the) kidney yin & 1,490 \\
\hline $\begin{array}{l}\text { 7. (qi stagnation of/liver/phlegm and/heart) blood stasis } \\
\text { (syndrome) }\end{array}$ & 1,335 \\
\hline 8. (the/in the/compared with the/a) control group & 1,263 \\
\hline $\begin{array}{l}\text { 9. (descending of/the descending of/the/tonify/tonifies) } \\
\text { lung qi (deficiency) }\end{array}$ & 1,236 \\
\hline 10. (the) treatment principle (of) & 1,216 \\
\hline 11. (heart/stomach/kidney) qi deficiency & 1,205 \\
\hline 12. (Zhi) Gan Cao & 1,203 \\
\hline 13. (liver) qi stagnation & 1,188 \\
\hline 14. (spleen/spleen and kidney/heart) yang deficiency & 1,140 \\
\hline $\begin{array}{l}\text { 15. (the/tonify/tonifies/ascending of/deficiency of/the } \\
\text { ascending of /deficient) spleen qi }\end{array}$ & 1,119 \\
\hline 16. Fu Ling & 1,073 \\
\hline 17. (the) clinical manifestations (of) & 1,050 \\
\hline 18. (chest/chronic) painful obstruction & 1,030 \\
\hline 19. Dang Gui & 960 \\
\hline 20. in the treatment (of) & 959 \\
\hline
\end{tabular}


A close look at the technical lexical bundles in Table 5.18 shows that there are three categories of technical lexical bundles in the discipline of TCM: Category 1 with all constituents being loan words (e.g., Gan Cao, Fu Ling, Dang Gui); Category 2 with at least one of the constituents being a loan word (e.g., kidney yin, lung qi, qi stagnation); and Category 3 with no constituent being loan word (e.g., damp heat, clinical manifestations, treatment principle).

Table 5.19 presents a detailed composition of the TCM technical lexical bundle list with number of items and examples in each category provided. The first category of technical lexical bundles consists of 159 entries, which contains 132 full names of herbs (e.g., Gan Cao, Fu Ling), 9 herbal formulae (e.g., Shen Qi Wan, Er Chen Tang), and 18 essential terms (e.g., yuan qi, san jiao) that do not have English equivalents. This set of lexical bundles characterize the discipline of TCM as they cannot be commonly seen in other specialized disciplines. It is interesting that all lexical bundles in this category are nominal structures, suggesting that the use of nominal expressions is a key and ubiquitous feature of TCM texts. Furthermore, some technical lexical bundles can create the subject knowledge in a way that the single word might not be able to. For example, ling itself occurred frequently in the TCM Corpora and in the TCM technical word list, but the meaning was unclear by itself unless it was combined with other loan words such as fu. Fu ling is a very commonly used herb medicine, which can be found in Table 5.18. This finding indicates that some technical words need to be learned in combinations rather than in isolation. In this regard, the technical lexical bundle list is a good supplement to the TCM technical word list.

Table 5.19: Composition of the TCM technical lexical bundle list

\begin{tabular}{|l|l|l|l|}
\hline Category & Constituents & $\begin{array}{l}\text { Number of } \\
\text { items }\end{array}$ & Examples \\
\hline Category 1 & All loan words & 159 & Fu Ling, Shen Qi Wan, yuan qi \\
\hline Category 2 & $\begin{array}{l}\text { Combination of loan } \\
\text { and English words }\end{array}$ & 89 & kidney yin, deficient qi, rectify the qi \\
\hline Category 3 & All English words & 650 & $\begin{array}{l}\text { clinical manifestation, nourish liver } \\
\text { blood, Sclerotium Poriae Cocos }\end{array}$ \\
\hline
\end{tabular}


TCM technical lexical bundles at Category 2 consist of both loan words and English words, such as kidney yin, deficient qi, and rectify the qi (see Table 5.19). There are 89 lexical items (out of 898) of this type in total. Among them, the most common pattern is also nominal structures (e.g., kidney yin, deficient qi), which is comprised of 72 items. They are of crucial importance in developing abstract and complex technical concepts in TCM especially concepts which do not have equivalents in Western medical science. For example, when yin (noun) and kidney (noun) are combined as kidney yin, a complex technical concept is established, which is considered to be "the foundation of all the Yin energies of the body" and "is the fundamental substance for birth growth and reproduction" (Maciocia, 2005, p. 153). This implies that, in TCM, kidney has the function of governing birth, growth and reproduction in addition to the function of controlling urine, whereas kidney has no such extended meaning in Western medical science. The presence of such unusual combination of nouns thus indicates that kidney was used with an extension of the mainstream meaning via metaphorical/figurative use. In other words, that the metaphorical use of some technical words may be signaled by the presence of unusual combinations is interesting. In addition to most common noun combinations for constructing complicated concepts, there are 13 items consisting of a 'verb + noun' structure (e.g., nourish yin, move liver qi). This shows the collocational behavior of the important concepts in TCM, and illustrates how the nouns and noun combinations relate to and interact with each other.

It can also be seen from Table 5.19 that a vast majority of the technical lexical bundles (650 out of 898 items) in English-medium TCM texts are in Category 3. These multiword unit combinations are made up of English words. As shown in Table 5.20, the nominal structures (e.g., damp heat, clinical manifestations) are dominant (551 items), followed by verb + noun structures (e.g., nourish liver blood, clear heat). The nominal structures serve to construct complicated concepts of TCM and also form Latin names (e.g., Sclerotium Poriae Cocos, Radix Angelicae Sinensis) of the herbs. For example, Sclerotium Poriae Cocos is the English name, or to be more precisely, the Latin name, of Fu Ling. Similar to herb names in the form of loan words, this kind of lexical bundles can supplement the single words Sclerotium, Poriae, Cocos by making them into meaningful units. This finding further supports the view that some technical words need to be learnt in combinations. In addition to the combinations mentioned above, propositional 
structure (e.g., in the lung, in vitro) can also be commonly seen (40 items), which usually indicate the place, the time, the condition of the nouns or noun combinations relate to or interact with each other.

Table 5.20: Summary of structures of lexical bundles at Category 3

\begin{tabular}{|l|l|l|}
\hline Structure & Number of items & Examples \\
\hline Nominal structure & 551 & damp heat, Radix Angelicae Sinensis \\
\hline Verb structure & 59 & nourish liver blood, clear heat \\
\hline Prepositional structure & 40 & in the lung, in vitro \\
\hline
\end{tabular}

Overall, one interesting finding concerning the TCM technical lexical bundle list is the dominance of nominal expressions in each category of the bundles. In other words, noun combinations are key resources in constructing disciplinary knowledge. This finding reflects the information-dense nature of TCM texts as a highly specialized academic discipline. Another interesting finding is that the full names of herbs in the lexical bundle list, either in the form of loan words (Romanized Chinese) or Latin words, can complement the TCM technical word list which separates full names into single words in some cases. The third finding is that the metaphorical use of technical words as found in Section 5.5.2 can be signaled by the presence of unusual noun combinations (especially loan word + English word combination, and vice versa) identified in the technical lexical bundle list. The technical lexical bundle list further reflects the lexical complexity of the TCM discourse. The full list of the technical lexical bundles in each category can be found in Appendices 11-13. The indications of the findings will be further discussed in the discussion chapter (Section 7.2.2.3).

\subsection{Summary of main findings and rationale for next study}

To sum up, this study has four main findings. The first finding concerns the technical words identified in BASEWRD 1-25 of BNC/COCA lists. There were 4,368 word types, which covered $33.5 \%$ of the total tokens in the TCM Corpora. An analysis of semantic features of technical words from BASEWRD 1-25 shows that those words fall into four distinctive groups to lexicalize the subject knowledge of TCM. Importantly, the technical words used with specialized and metaphoric meanings may cause comprehension problems even though these word forms are generally known by learners. However, 
empirical evidence is needed to support this claim. This indicates a need to investigate learners' understanding of these technical words, which will be the main focus of Study 3. The second main finding is the overall coverage of technical words, which was $39.46 \%$. Such high density of technical words in the TCM texts indicates it is challenging to read such texts. In Chapter 7, this finding will be used in Section 7.2.1 to highlight the importance of technical vocabulary in specialized English.

The third finding is related to the development of the TCM technical word list, which consisted of 2,778 word types based on keyness, meaning, and frequency criteria using the TCM Corpora. The list was divided into three sub-lists according to frequency of occurrences, and each list was divided into three levels to cater for the needs of TCM learners from different linguistic backgrounds and vocabulary levels. In total, they covered $36.64 \%$ and $31.43 \%$ of the tokens in the whole TCM Corpora and the validating TCM corpus. These figures were considerably higher than the coverage of the TCM technical word list in Western Medical Corpora, and general written English corpus, suggesting that it is a truly technical word list in TCM. In Chapter 7, this finding will be drawn together with other findings to highlight the value of discipline-specific word lists in specialized English teaching and learning in Section 7.4.

The last main finding of this study concerns the TCM technical lexical bundle list, which contained 898 technical lexical bundles. The noun bundles were found to dominate in the list, which reflect the nature of academic texts in science disciplines. The importance of the TCM technical bundles list is highlighted by the fact that it can supplement the TCM technical single-word list, indicating that some technical words need to be learnt in combinations. These findings will be used in Section 7.2.2.3 to discuss the importance of technical vocabulary beyond single word units in subject knowledge construction.

It should be acknowledged that both the TCM word list and lexical bundle list are very long listings of lexical items, which provide useful targets of eventual learning goals for TCM learners. However, how to achieve such ambitious learning goals remains an issue. It is actually a practical issue for most word list studies. The next study therefore makes an attempt to investigate learners' understanding of technical words identified in the first 
two studies. Such investigation can provide useful insights into the selection of items which merit attention or more explicit attention in the valuable learning time. 


\section{Chapter 6 Investigating learners' understanding of technical vocabulary}

\subsection{Introduction}

From Chapters 4 and 5, a relatively comprehensive understanding of the nature of vocabulary, especially technical vocabulary, in the discourse community of TCM was obtained. The present chapter explores TCM learners' understanding of technical words identified in Study 1 and Study 2. The exploration has two aims. The first aim is to investigate whether TCM learners have difficulty in understanding the technical words in their field of study. The second aim is to investigate whether Western TCM learners and Chinese TCM learners understand the technical words in the same way given the different linguistic and cultural backgrounds they are from and the unique characteristics of technical vocabulary in TCM. The investigation is based on the research questions (RQs) listed below:

1. Do the TCM learners have difficulty in understanding technical vocabulary in their field of study?

If so, what kind of technical vocabulary poses difficulties to the learners, and why does it pose challenges?

2. Do Western-background TCM learners understand technical words in TCM in the same way as Chinese-background TCM learners?

2a. If not, which kind(s) of technical words manifest this divergence between the two groups' understanding (the most)?

2b. What factors help account for the divergence, if any, in the two groups' understanding of the technical words?

This third study (henceforth Study 3) serves a diagnostic purpose to identify 'problematic' technical words and find out what makes them problematic. This could be followed up by the development of pedagogic instruments to help remedy the attested problems (if any). Ultimately, it seeks to provide insights into the pedagogical merits of teaching certain technical words in the long-listing technical word list developed from Study 2, taking into account learners' specific needs and lacunae in their knowledge. Further, given the cultural differences between Chinese and Western conceptions of body and health, it is 
possible that some technical terms in TCM, either borrowed intact from Chinese or substituted by an English-word translation, activate a network of associations in Western background TCM students that only partially overlaps with that of Chinese background students. Therefore, it is useful to investigate the understanding of these terms between Chinese and Western learners since they might not be semantically transparent to learners from different cultural and linguistic backgrounds, and thus might pose potential difficulties to the learners. The hypothesis is that, if divergence between the Western and Chinese TCM learners' understanding of technical words is found, it will often be attributable to cross-cultural differences in metaphoric conceptions of the body and matters of health and well-being between Chinese and Western society. The theoretical background for this general hypothesis is provided by the Cognitive Linguistic treatment of word meaning as 'encyclopedic': A word activates in the language user's mind much more than its 'denotation' or dictionary meaning (Littlemore, 2009). Rather, it activates a network of associations connected to the language user's experience of the word as it is socially and culturally grounded (Littlemore, 2009).

The remaining part of the present chapter focuses on the methodology, results and findings of Study 3. Specifically, Section 6.2 describes the methodology of the present study, while Section 6.3 presents the results and findings. Section 6.4 concludes the current chapter with a summary of the main findings.

\subsection{Methodology}

This section starts with a description of the participants in this study, followed by the description of the instruments (word association task and retrospective interview) used in this study and the detailed procedure of data collection. Section 6.2 ends with a description of how the data were analyzed.

\subsubsection{Participants}

Twenty-two TCM university students agreed to take part in this study. These participants came from two groups with similar sizes: 12 TCM students from a Chinese TCM university and 10 TCM students from a New Zealand TCM institution at the tertiary level. However, the data from one Chinese participant was excluded because this participant did not appear to know most of the words, either cue words or association choices, in the word association task, including common words such as wind. This is surprising given that all 
participants were university students. Consequently, the data from 11 Chinese TCM learners were used for the analysis.

The 11 Chinese TCM learners were 19 to 25 years old, and their medium of instruction is mainly Chinese. They had studied English for over eight years and had a high score of English on the College Entrance Examination to enter that university, and so they can be regarded as advanced English learners. It is important to mention that six of them had also had two years of English training as English major students at university prior to undertaking TCM study. Table 6.1 provides detailed information about the 11 Chinese TCM learners' gender, their specialization within TCM, year of studying TCM, and whether they had English training at university prior to TCM. As can be seen in the third column of Table 6.1, the Chinese participants majored in three different specialisms TCM as a degree course, Chinese herbal medicine as a degree course, and acupuncture and moxibustion as a degree course.

Table 6.1: Chinese TCM learners' demographic information

\begin{tabular}{|l|l|l|l|l|}
\hline Participant & Gender & Specialization & $\begin{array}{l}\text { Year of } \\
\text { studying } \\
\text { TCM }\end{array}$ & $\begin{array}{l}\text { English } \\
\text { training prior } \\
\text { to TCM }\end{array}$ \\
\hline CP1 & Female & TCM & 5 & Yes \\
\hline CP2 & Female & TCM & 4 & Yes \\
\hline CP3 & Female & Chinese Herbal Medicine & 3 & No \\
\hline CP4 & Male & Acupuncture and Moxibustion & 2 & Yes \\
\hline CP5 & Female & TCM & 1 & No \\
\hline CP6 & Female & TCM & 2 & No \\
\hline CP7 & Female & Chinese Herbal Medicine & 3 & No \\
\hline CP8 & Female & Acupuncture and Moxibustion & 2 & Yes \\
\hline CP10 & Female & Acupuncture and Moxibustion & 2 & Yes \\
\hline CP11 & Female & Acupuncture and Moxibustion & 2 & Yes \\
\hline CP12 & Female & Chinese Herbal Medicine & 4 & No \\
\hline
\end{tabular}


It needs to be clarified that the year of studying TCM in Table 6.1 does not necessarily mean the participants' year of university study because some programs have two years of intensive English training at a language university prior to starting the actual TCM program. During those two years, the students only study English courses as English major students. Therefore, if a participant is in the second year of studying TCM, it might mean that he/she is a year four university student. Other students, however, only take one compulsory college English course each semester, and might take elective English for Chinese Medical Purposes or English for Medical Purposes courses depending on their choices.

Table 6.2: New Zealand TCM learners' demographic information

\begin{tabular}{|l|l|l|l|}
\hline Participant & Gender & Specialization & Year of learning TCM \\
\hline NP1 & Female & Acupuncture & 4 \\
\hline NP2 & Female & TCM & 3 \\
\hline NP3 & Male & Acupuncture & 1 \\
\hline NP4 & Female & Acupuncture & 3 \\
\hline NP5 & Female & Acupuncture & 3 \\
\hline NP6 & Male & Acupuncture & 4 \\
\hline NP7 & Female & Acupuncture & 2 \\
\hline NP8 & Male & Acupuncture & 2 \\
\hline NP9 & Female & Acupuncture & 2 \\
\hline NP10 & Female & Acupuncture & 2 \\
\hline
\end{tabular}

The TCM learners from New Zealand TCM institution were between the ages of 23 and 53 and they were studying TCM to obtain a Bachelor's degree. The Bachelor's degree in TCM in New Zealand is a four-year program with English being the medium of instruction. The New Zealand participants were TCM learners from year one to year four. They were nine native English speakers from New Zealand, America and the U.K., and one Taiwanese. The Taiwanese participant had lived in New Zealand for over 20 years, so she was considered an English speaker for the purpose of this study (although she is a native Chinese speaker). One of the English native speaking participants has Chinese parents, and thus has some knowledge of spoken Chinese. This participant and the 
aforementioned Taiwanese participant were called Western TCM learners with Chinese background. It should be noted that, in this study, the labels 'Chinese background learners' and 'Western background learners' were used to refer to learners studying TCM in China and learners studying TCM in New Zealand respectively. This categorization is oversimplistic since learners can definitely have multicultural backgrounds. Table 6.2 presents detailed information about the New Zealand learners' gender, specialization with TCM, and year of learning TCM.

The number of participants in this study is relatively small. However, this study does not attempt to establish response norms for the word association task, nor does it aim to draw conclusions on the basis of quantitative analyses. Rather, it is an exploratory study which aims to investigate TCM learners' understanding of technical vocabulary, identify sources of comprehension problems, and possible cross-linguistic and cross-cultural differences in word comprehension between aforementioned two groups of TCM learners using two rich sets of qualitative and quantitative data.

\subsubsection{Word association task}

A word association task was used in this study for two reasons. First, it can indicate how well the participants understand the technical words, since Read (2012) and Schmitt, Ng, and Garras (2011) have demonstrated that it can reliably measure how well cue words are mastered. Second, the association responses offered by the participants would serve as input for further interview interaction in order to obtain a fuller picture of what (background/subject) knowledge drives the given associations. In the following subsections, the selection of the format of word association task, cue items, and associations are presented in turn.

\subsubsection{Format of the word association task}

In general, there are two formats of word association tasks - the standard format is to ask the participants to supply response words (free association) and the more recent and innovative format is to ask the participants to select given associations (selected association) (Read, 2012). A selected association task was used in this study for two reasons (see Figure 6.1 for the example of the task instructions). First, to decide which format of the word association task to use in the present study, a pilot study was conducted with one TCM learner who is a Chinese speaker but has relatively high level of 
English proficiency. The pilot study suggested that the free association task was too challenging because it required the participants to supply responses and she could only supply responses to five out of twenty given cue words. Second, Meara (2009) found that second language (L2) learners provided less consistent and interpretable responses than the native speakers in the free association task, which led to less promising findings in gaining an understanding of L2 learners' lexical network.

\section{Figure 6.1: Instructions for the word association task}

This task contains target 20 words. Each target word (in bold) has eight (8) options. Please choose four words of the eight options which you think are most closely associated to the following words in the context of Traditional Chinese medicine (TCM). Put a tick $(\sqrt{ })$ in the box for each option you choose.

\begin{tabular}{|l|c|}
\hline Here is an example: & You might answer the task like this: \\
joint & joint \\
$\square$ A. qi & $\checkmark$ A. qi \\
$\square$ B. tendon & $\checkmark$ B. tendon \\
$\square$ C. skeleton & $\square$ C. skeleton \\
$\square$ D. blood & $\checkmark$ D. blood \\
$\square$ E. pain & $\square$ E. pain \\
$\square$ F. heart & $\square$ F. heart \\
$\square$ G. shoulder & $\square$ G. shoulder \\
$\square$ H. hepatitis & $\square$ H. hepatitis \\
\hline
\end{tabular}

Think aloud: As you make your choices, I would like you to talk about your choices and why you chose (or did not choose) each one.

The selected association task was based on Read's (1998) version of word association test, in which the participants were asked to choose four associates out of eight options provided. Though similar to Read (1998), modifications were made to the association task so as to ensure a closer fit with the aim of the present study. For example, in the instruction, the participants were asked to choose four associations which they think are most closely associated with the cue words in TCM context. This decision was made 
because, according to Study 2, some technical words are metaphoric or polysemous in the context of TCM. Therefore, knowing the literal or general meaning of these words does not guarantee that they understand the technical meaning of these words in TCM. By asking them to choose what they felt to be the most closely associated words, their indepth understanding of these technical words can be revealed. More information is provided in Section 6.2.2.3 about associations and distractors.

The eight-option format was adopted since, compared to a six-option format, it is less susceptible to guessing (Schmitt, Ng, et al., 2011). In addition, Schmitt (2000) also suggested that the eight-option format might be more suitable for high-frequency polysemous words. Considering that a considerable number of technical words in TCM are high-frequency words which are often polysemous or metaphorical (see Chapter 5), the eight-option format is the most suitable format to this study. It is also a commonly used format in word association tests (Qian, 1999; Qian \& Schedl, 2004; Read, 1993, 1998; Schmitt, Ng, et al., 2011).

In total, the word association task consisted of 20 items (see Appendix 14 for the task example). The cue words of the association task were limited to 20 items for considerations of time. The association task as such was only a part of the interview procedure (see Section 6.2.4 for details), where the association responses served as prompts in the subsequent retrospective interview. Twenty items allowed the interviews to be finished in one hour. Longer interviews were deemed undesirable because of the risk of fatigue on the part of the interviewees. That the interview would be manageable in an hour was estimated by piloting it with one former TCM learner from China. In the pilot study, the 20-item word association task and the interview was finished in one hour. In the next part, how these 20 cue words were selected is described.

\subsubsection{Target cue words}

The sheer number of technical words in the TCM technical word list (see Chapter 5 for more information) indicates that a principled manner of selecting items to be focused on in the present study is required. To select such items, two properties of technical words - frequency banding and semantic transparency/opacity - were taken into account as complementary parameters (as shown in Figure 6.3). This decision was made because, as 
was found out in Study 1 and 2, technical words can be from any frequency bands and those from high-frequency bands and lower-frequency bands may be of different familiarity to the learners depending on the learners' proficiency level of English. Therefore, the frequency band of a technical word is an important property that needs to be considered. Another property is that some technical words are metaphoric or polysemous in a specific discipline, especially those from Schmitt and Schmitt's (2014) high-frequency vocabulary band. They are seemingly familiar but might take a very specialized sense which may not be known by a learner at all, and thus create a range of comprehension problems that learners are not always aware of. Such words were regarded as opaque technical words in this study. Watson Todd (2017) also argued that opacity of technical words is an important property, which causes problems if the learners have to deal with it autonomously.

The idea of selecting technical words according to the parameters of frequency and transparency/opacity was borrowed from Martínez (2013). In a study to select multiword expressions for explicit instruction, Martínez (2013) proposed an innovative framework based on the frequency and transparency/opacity of given multiword expressions (see Figure 6.2). It is a useful framework which allows English teachers to make informed decisions about what to teach in classrooms.

Figure 6.2: Martinez's (2013, p, 190) Frequency-Transparency Framework

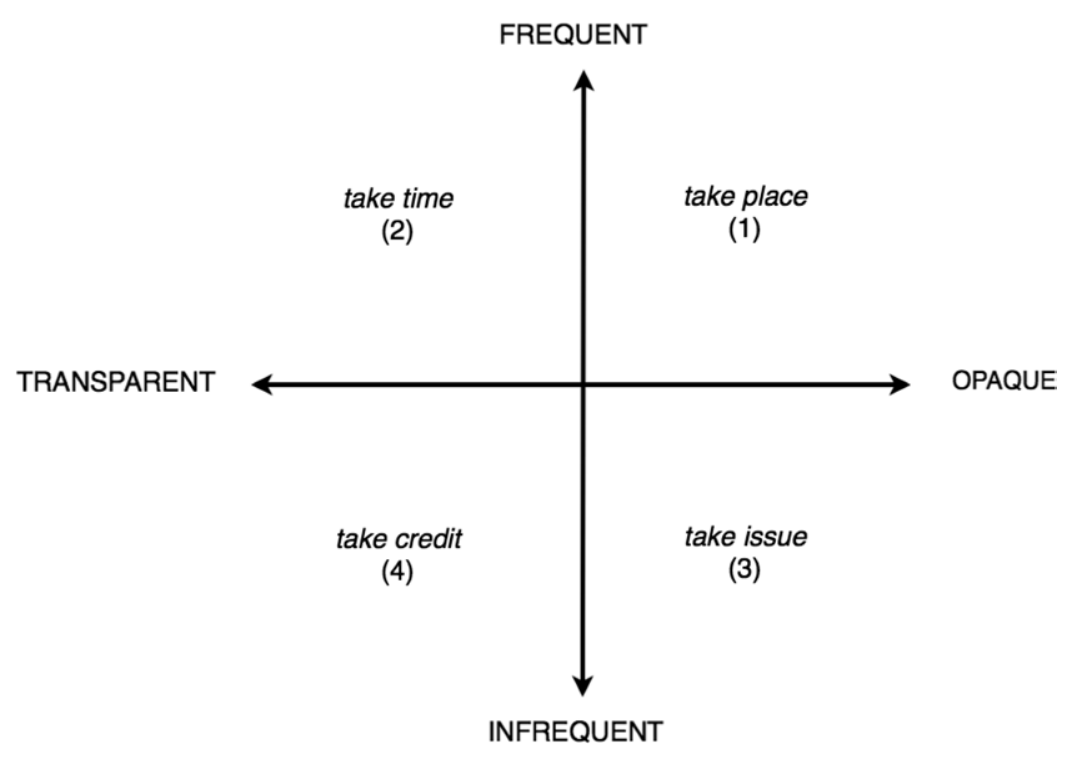


Similarly, the TCM technical words were divided into four groups based on a FrequencyTransparency Framework as presented in Figure 6.3. However, frequency here means the frequency-banding of a word in the BNC/COCA base word lists, whereas transparency refers to the extent to which the specialized sense of a technical word departs from its literal/general meaning.

Figure 6.3: The Frequency-Transparency Framework for technical words

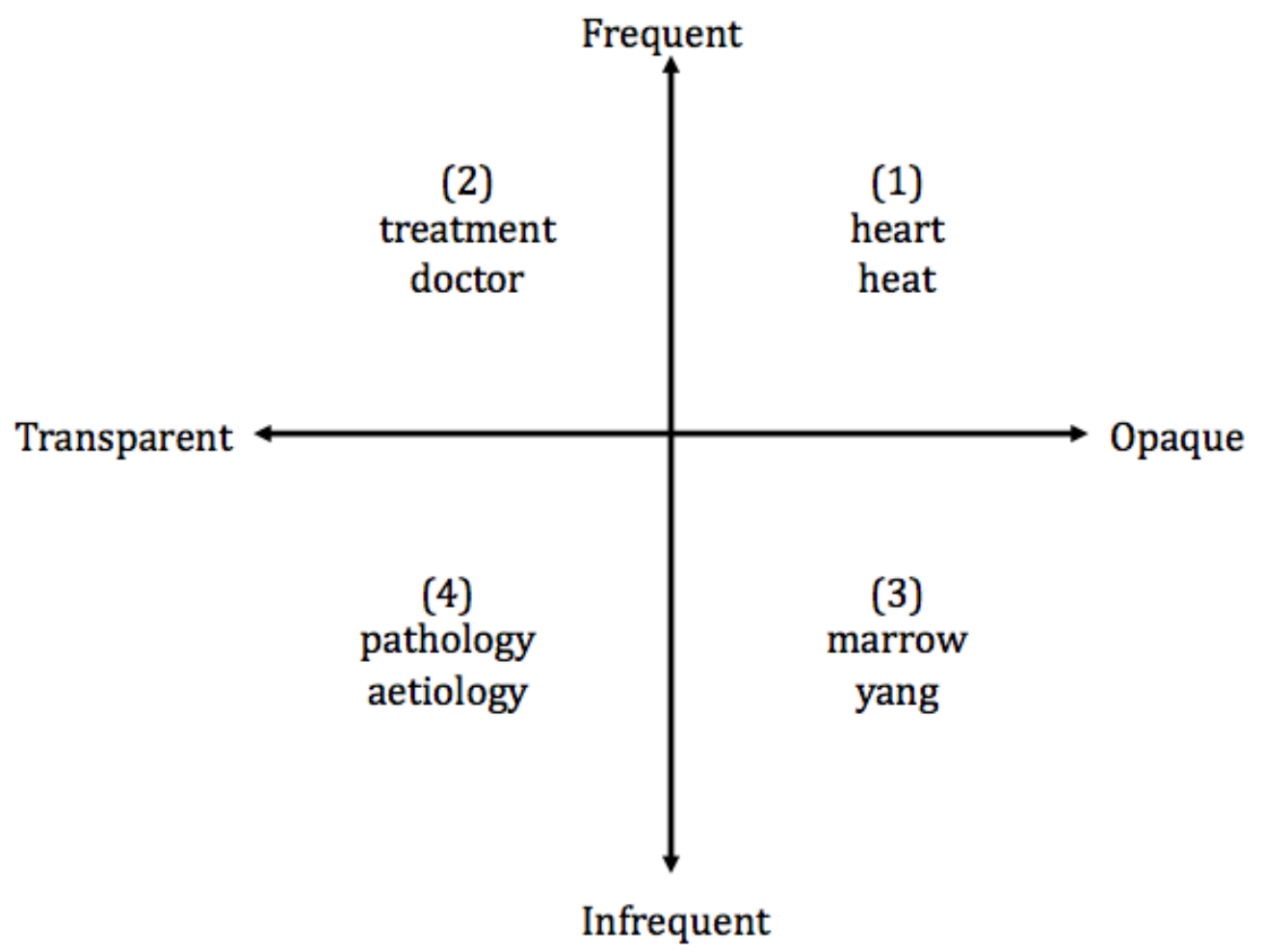

Figure 6.3 presents an intersection of continua based on frequency and transparency of technical words. The vertical continuum represents frequency while the horizontal continuum represents semantic opacity. On the vertical continuum, technical words which are from the first to the third 1,000 word families of the BNC/COCA word lists lie on the upper side and those in the lower frequency bands than the third 1,000 on the lower side. The cut-off point at 3,000 BNC/COCA level, which was proposed by Schmitt and Schmitt (2014) as the high-frequency vocabulary, was adopted since it "represents an important milestone in language development" (Schmitt \& Schmitt, 2014, p. 492) and advanced English learners are highly likely to be familiar with these words. In addition, even though the high-frequency vocabulary only involves three base words lists with 
3,000 word families, it covers $75.3 \%$ of the total tokens and involves 11,449 word types out of the total 40,070 word types that appear in the whole TCM Corpora (see Chapter 5 for details). As shown in Figure 6.3, heart, heat, treatment, and doctor are from the first 3,000-word-family bands so that they were placed on the upper side of the intersection of the continuum. Correspondingly, pathology, aetiology, marrow and yang were located on the lower side since they are from the 3,000+ BNC/COCA word family levels.

On the horizontal continuum, items likely to be opaque were located on the right side whereas those likely to be transparent were on the left side. The first indicator of opacity is whether the technical sense of a word differs from its literal/general sense. In other words, if a word is metaphorical or polysemous in this specialized field, this word was considered as opaque. For example, heart means "a hollow muscular organ of vertebrate animals that by its rhythmic contraction acts as a force pump maintaining the circulation of the blood" (heart, n.d.), whereas in TCM it refers to the organ that is situated in the chest whose main functions are to govern blood and to house the mind (Maciocia, 2005). This means that "the state of heart (and blood) will affect the mental activities including the emotional state" of a human being (Maciocia, 2005, p. 109). The concept of heart is therefore quite different in TCM from that of the general concept of heart because of the differences between Chinese and Western conceptions of body and health. Newcomers of this field might not even be aware of such conceptual differences, and as a result the technical use of this word in TCM may be opaque to them.

The second indicator is whether a technical word is a loan word which has been borrowed intact from Chinese. For instance, yang is known to people as an important and distinctive concept of Chinese philosophy, which refers to "the masculine active principle in nature that in Chinese cosmology is exhibited in light, heat, or dryness and that combines with yin to produce all that comes to be" (yang, n.d.). Due to the fact that TCM employs a unique theoretical system and a holistic approach to describing both health and diseases on the basis of Chinese philosophy of Yin-Yang theory, Yin and Yang became core technical concepts in TCM. Understanding the application of Yin-Yang in TCM is of crucial importance. However, this might not be easy to learners who are from a nonChinese background. Second, Yang is a commonly seen family name in Chinese, and these diverse uses of the word may make its technical meaning in more opaque especially for 
non-Chinese speaking learners. For these reasons, loan technical words were considered as likely to be opaque as well. As shown in Figure 6.3, heart, heat, marrow and yang were placed on the right (opaque) side because heart, heat, and marrow have specialized senses in TCM which differ from their literal/general meanings and yang is a loan word borrowed from Chinese directly.

Following these principles, the most frequent 200 technical words on the frequencybased TCM technical word list from Study 2 were allocated into the four quadrants: Quadrant 1 with relatively frequent and opaque technical words, Quadrant 2 with relatively frequent and transparent technical words, Quadrant 3 with relatively infrequent and transparent technical words, and Quadrant 4 with relatively infrequent and opaque technical (see Appendix 18 for examples). Five technical words from each Frequency-Transparency category were randomly selected for the word association task and used as the prompts in the retrospective interviews (see Appendix 19 for interview guide). The cue words selected from each category were mixed in the word association task to ensure that not all the low frequency words come together, which might have caused frustration in the participants.

\subsubsection{Associations and distractors}

When the original set of cue words was selected, the associates and distractors were carefully chosen as provided options in the word association task. The associations were selected based on Fitzpatrick's (2006) categories of association responses, which contained very detailed subcategories of association in each category (see Table 6.3). Modifications, however, were made to Fitzpatrick's (2006) association categories so as to ensure a closer fit with the aim of word association task used in the present study. Only the meaning and position-based (similar to syntagmatic) associations were used because the word association task was used as prompts to explore learner's deeper understanding of technical words. When selecting the position-based associations, the TCM technical lexical bundle list (see Section 5.5.7 for details) acted as a guide. The meaning-based associations could be any of the six subcategories in Table 6.3. Each item contained a mixture of association options. 
Table 6.3: Categories and subcategories of word association responses (Adapted from Fitzpatrick, 2006, p. 131)

\begin{tabular}{|c|c|c|}
\hline Category & $\begin{array}{l}\text { Subcategory ( } \mathrm{x}=\text { cue } \\
\text { word, } \mathrm{y}=\text { response } \\
\text { word) }\end{array}$ & $\begin{array}{l}\text { Definition ( } \mathrm{x}=\text { cue word, } \mathrm{y}=\text { response } \\
\text { word) }\end{array}$ \\
\hline \multirow{6}{*}{$\begin{array}{l}\text { Meaning- } \\
\text { based } \\
\text { association }\end{array}$} & Defining synonym & $\mathrm{x}$ means the same as $\mathrm{y}$ \\
\hline & Specific synonym & $\mathrm{x}$ can mean $\mathrm{y}$ in some specific contexts \\
\hline & $\begin{array}{l}\text { Hierarchical/lexical } \\
\text { set relationship }\end{array}$ & $\begin{array}{l}\mathrm{x} \text { and } \mathrm{y} \text { are in the same lexical set or are } \\
\text { coordinates or have a meronymous or } \\
\text { superordinate relationship }\end{array}$ \\
\hline & Quality association & $\mathrm{y}$ is a quality of $\mathrm{x}$ or $\mathrm{x}$ is a quality of $\mathrm{y}$ \\
\hline & Context association & $\mathrm{y}$ gives a conceptual context for $\mathrm{x}$ \\
\hline & $\begin{array}{l}\text { Conceptual } \\
\text { association }\end{array}$ & $\mathrm{x}$ and $\mathrm{y}$ have some other contextual link \\
\hline \multirow{2}{*}{$\begin{array}{l}\text { Position- } \\
\text { based } \\
\text { association }\end{array}$} & $\begin{array}{l}\text { Consecutive } \mathrm{xy} / \mathrm{yx} \\
\text { collocation }\end{array}$ & $\mathrm{x}$ collocates with $\mathrm{y}$, or $\mathrm{y}$ collocates with $\mathrm{x}$ \\
\hline & $\begin{array}{l}\text { Phrasal xy/yx } \\
\text { collocation }\end{array}$ & $\begin{array}{l}\mathrm{y} \text { follows } \mathrm{x} \text { in a phrase, or } \mathrm{x} \text { follows } \mathrm{y} \text { in a } \\
\text { phrase }\end{array}$ \\
\hline
\end{tabular}

To make the word association task less susceptible to guessing, one to two distractors were provided to each item as options. For the same reason, the distractors were selected from the TCM technical word list but they were selected on the basis that they had no direct semantic link with the cue word. The reason for offering only one to two distractors is because this task, different from Read (1998), asked the participants to select four associations which they think are most closely associated with the cue words. Take joint for example.

\section{joint}
$\square$ A. qi
B. tendon
$\square$ C. skeleton
$\square$ D. blood
E. pain 

$\square$ F. heart
$\square$ G. shoulder
$\square$ H. hepatitis

In TCM, joints are "more than just anatomical entities, they have an important function with regard to the circulation of Qi and Blood, with several implications in pathology" (Maciocia, 2005, p. 752). As shown above, tendon and skeleton are paradigmatic associations which are from the same word class as joint, while qi and blood are meaningbased associations which form the conceptual meanings of joint. Pain (joint pain) and shoulder (shoulder joint) are syntagmatic associations which can be found in the TCM technical lexical bundle list. In this example, heart and hepatitis are distractors. Only when the learners understand joint in TCM well can they associate joint with qi and blood. Otherwise, there are still four other words which can be associated with the cue words. Choosing words other than qi and blood can also show that the learners understand the literal meaning of joint. The selection process can thus give us a better chance to know whether learners understand the extended metaphoric meaning of the target cue words in the TCM context. To sum up, the selection of associates is a measure of how well the target cue words are known, and the process of selecting the associates reflects the knowledge that drives the associations.

\subsubsection{Piloting the word association task}

When all the cue words, associations, and distractors were selected, the draft word association task was piloted on one previous TCM learner and one native English speaker who has no knowledge of TCM. Necessary revisions were made. For example, stroke was one of the cue words from the category of Quadrant 2 (frequent and transparent). It was replaced by protein because it was found that stroke was ambiguous. The English native speaker interpreted 'stroke' as a verb instead of a noun, and interpreted it as 'to rub gently in one direction' or 'the act of striking' (stroke, 2018). However, in medicine, it usually means "a serious illness caused when a blood vessel in your brain suddenly breaks or is blocked" (stroke, n.d.). It is a case of homonymy. A sample of the final word association task is given in Appendix 14. 


\subsubsection{Retrospective interview}

The association responses offered by the participants served as input for interview interaction in order to obtain a fuller picture of what knowledge drove the given associations. In addition, the interviewer can probe how well the participants really know the target words by asking them to justify their choices. It can thus increase the validity of the results generated from the word association task.

The retrospective interview was chosen because it is a useful instrument to explore not only for complete answers but also for responses about complicated and in-depth ideas and concepts behind the answers (Cohen, Manion, \& Morrison, 2007). With students from Chinese universities, the word association task was written in English, but they could choose either Chinese or English in the think-aloud interview interaction to help them express their ideas freely (see Appendix 19 for interview guide). Unsurprisingly, all Chinese participants chose Chinese as the language in the interview interaction. The interviews were conducted in English with the non-Chinese-background TCM learners.

\subsubsection{Procedure}

In the previous subsections, the participants, instruments, and task items have been described. This subsection describes the procedure by which the data were collected. The data collection was approved by the Human Ethics Committee of Victoria University of Wellington, New Zealand (see Appendix 15). Prior to data collection, an official invitation was sent to TCM undergraduates through different channels such as WeChat and email. Participants who were interested in participating in this study contacted the researcher via WeChat message or email. The researcher then set up one-on-one meetings with each participant in person.

At the first stage of the interview, questions regarding their educational level, specialism, L1, and other relevant demographic information were asked. For example, 'what's your name?' and 'how many years have you been learning TCM?'.

In the second stage, the participants were invited to perform a paper-and-pencil word association task according to the given instruction as shown above in Figure 6.1. The instruction was written in English because the participants in this study are either 
advanced English learners or native speakers. After they read the written instruction, more detailed instruction was supplemented orally to ensure that the participants understood the instruction. For example, the reasons why A. qi, B. tendon, D. blood and E. pain were chosen was provided in the example: qi and blood were chosen because joints have a function related to the circulation of qi and blood in TCM, tendon was chosen because it is close to joints from an anatomical point of view, and pain was chosen because joint pain is commonly shown in TCM texts. Participants were not given time constraints for this task, but the task was answered face-to-face with the researcher and without access to dictionaries or other related texts. All participants finished each task item within one minute.

The participants were asked to share their thinking processes regarding how they arrived at their answers immediately after they finished each item in the association task. Questions such as 'why did you choose A?' and 'why do you associate this word with B?' were asked to supplement their think-aloud process. This means that immediately after the participants answered each item, they were asked to reflect on the reasons behind their responses to the association task.

At the last stage, when the task items and related questions were all answered, the participants were asked a few questions about whether they had specialized English courses and, if so, when they had taken such courses. The whole interview process was audio recorded. If there were unclear issues related to the participants' answers or explanations, the researcher contacted the participants for further clarification.

\subsubsection{Data analysis}

The interviews were transcribed before scoring the task since they were important for deciding to the extent to which the cue words were understood. The word association task by each participant was scored using a 5-point scale for responses to each cue item, yielding a total maximum score of 100 points for this 20 -item task. The answers on each item were rated from 0 to a maximum score of 5 points according to the responses provided in the written task and the elaborations given in the retrospective interview, as specified in Table 6.4. 
Table 6.4: Scoring criteria for the word association task

\begin{tabular}{|c|c|c|}
\hline Degree & Points & Criteria \\
\hline Full knowledge & 5 & $\begin{array}{l}\text { Selected at least three associations related to TCM } \\
\text { and demonstrated full knowledge of the cue word in } \\
\text { TCM context }\end{array}$ \\
\hline \multirow{4}{*}{ Partial knowledge } & 4 & $\begin{array}{l}\text { Selected at least three associations related to TCM } \\
\text { and demonstrated some knowledge of the cue word } \\
\text { in TCM context }\end{array}$ \\
\hline & 3 & $\begin{array}{l}\text { Selected at least two associations related to TCM and } \\
\text { demonstrated a little bit of knowledge of the cue } \\
\text { word in TCM context }\end{array}$ \\
\hline & 2 & $\begin{array}{l}\text { Selected at least one association related to TCM and } \\
\text { demonstrated no knowledge of the cue word in TCM } \\
\text { context }\end{array}$ \\
\hline & 1 & $\begin{array}{l}\text { Selected association(s) unrelated to TCM but related } \\
\text { to literal/general meaning and demonstrated no } \\
\text { knowledge of the cue word in TCM context }\end{array}$ \\
\hline No knowledge & 0 & $\begin{array}{l}\text { Did not know the cue word at all or selected items } \\
\text { that could not be justified }\end{array}$ \\
\hline
\end{tabular}

On the 5-point scale, the reason to use the criterion of at least three associations related to a cue word in TCM is because, in some cases, the participants chose only three associations which they thought were most associative but also demonstrated full knowledge of the cue word. For example, the cue word cancer (see Appendix 14) had eight options in the sequence of A. tumour, B. toothache, C. malignant, D. stroke, E. lung, F. colorectal, G. gastric, H. chemotherapy, but one Chinese participant only chose A, C, H and explained that E, F, G were too narrowly focused because they were types of cancer (B and D were distractors). In this case, she demonstrated full knowledge of this word and provided valid reasons for not choosing the others. Thus, she received 5 points for this item. In most cases, the participants chose four or more associations when they demonstrated full knowledge of the cue word. 
The responses were scored according to the criteria in Table 6.4 by the researcher first. To ensure reliability, a second rater who is an expert in TCM translation was employed to score one participant's responses on all 20 items according to the criteria in Table 6.4. The agreement was at a high level of $95 \%$, and agreement on the $5 \%$ other items was reached through discussion. After the reliability of the scoring on the word association task was ascertained, the first rater scored all the tasks, and the second rater was consulted when there was any uncertainty. The total score of each respondent was then calculated and the results are reported in Section 6.3.1.

To answer RQ2, the typical associations of each participant group were identified and compared to find out if there is any divergence between the two groups' understanding of technical vocabulary in their field of study. The typical associations of each cue word in a participant group were the four associations that were selected by more participants in that group compared to other association options. The interview data were then analysed to decide whether divergence or convergence occurred between these two groups of TCM learners. This involved qualitative analysis through the description and interpretation of the way participants' background or learning experience may account for divergence or convergence in the associations from particular prompts in the word association task. The analysis follows the classic method for qualitative data analysis of Holliday (2010), which involves coding the data, determining the themes, constructing an argument, and collecting typical extracts to support the argument.

\subsection{Results}

So far in this chapter, the methodology of the present study has been described. This section first presents the results of the word association task of Chinese and Western background learners. Then, it reports on the comparison of typical associations between those two groups of learners, and the reasons why divergence or convergence of understanding occurred.

\subsubsection{Outcome of the word association task}

The first research question asks whether TCM learners have difficulty in understanding the technical words in their discipline of study. The present section answers this question from the perspectives of Chinese TCM learners and Western TCM learners respectively. 


\subsubsection{Performance of Chinese TCM learners in the word association task}

Table 6.5 provides the descriptive results of the word association task from the Chinese TCM learners. For the Chinese TCM students as a whole, the mean score of the word association task was 74.27 out of 100 , which suggests that they had good understanding of most technical words included in the task. At the same time, it also shows that these learners, on average, seemed to have insufficient knowledge of one fourth of the technical words included in the task.

Table 6.5: Descriptive statistics of Chinese learners' performance in the word association task $(\mathrm{N}=11)$

\begin{tabular}{|l|l|l|l|l|l|}
\hline Category & Total & Minimum & Maximum & M & SD \\
\hline $\begin{array}{l}\text { Frequent and opaque } \\
\text { technical words }\end{array}$ & 25 & 16 & 25 & 19.91 & 3.48 \\
\hline $\begin{array}{l}\text { Frequent and transparent } \\
\text { technical words }\end{array}$ & 25 & 16 & 25 & 23.64 & 2.94 \\
\hline $\begin{array}{l}\text { Infrequent and transparent } \\
\text { technical words }\end{array}$ & 25 & 0 & 25 & 12.27 & 9.27 \\
\hline $\begin{array}{l}\text { Infrequent and opaque } \\
\text { technical words }\end{array}$ & 25 & 7 & 25 & 18.45 & 7.09 \\
\hline All 4 categories & 100 & 40 & 98 & 74.27 & 21.12 \\
\hline
\end{tabular}

(Note: Total= maximum possible score; $\mathrm{M}=$ mean; $\mathrm{SD}=$ standard deviation)

Table 6.5 also demonstrates that the Chinese TCM learners generally scored higher for the technical words from high-frequency vocabulary bands (a total mean of $19.91+23.64$ $=43.55$ ) than those from the lower-frequency bands (a total mean of $12.27+18.45=$ 30.72). This result suggests that this group of TCM learners had better knowledge of the technical words from the high-frequency vocabulary bands than those from lowerfrequency bands of general English vocabulary. Of the two categories from the highfrequency band, the learners appeared to have better knowledge of transparent technical words such as skin and cancer $(\mathrm{M}=23.64)$ than the opaque technical words such as channel and mind $(\mathrm{M}=19.91)$. The interview data showed that four Chinese TCM learners had problems in understanding the extended metaphorical meaning of technical words in English, especially learners who did not take the English for Chinese Medical Purposes 
courses. For example, they understood the meaning of channel as in TV channel, but could not connect it with TCM. In this case, the learners showed an inability to understand the specialized meaning of general English words even though they have the subject knowledge and general English vocabulary knowledge. In terms of the technical words from low-frequency levels, the results in Table 6.5 indicate that Chinese TCM learners knew the opaque items such as tang and $q i(\mathrm{M}=18.45)$ better than the transparent items such as lumbar and diarrhoea $(\mathrm{M}=12.27)$ from the lower frequency vocabulary bands. These results are not surprising because the Chinese learners had an advantage in understanding loan words which are from the opaque and infrequent category.

Another important point to note from Table 6.5 is that there is a wide range of scores in the word association task, from students who displayed almost full knowledge of the target words (the highest score being 98 out of 100) to those who appeared to understand only a small percentage of them (the lowest score being 40 out of 100). This reflects a wide range of technical vocabulary knowledge among the Chinese TCM students. To examine the factors which might contribute to the differences, the Pearson $r$ correlation between the year of learning TCM and the scores in the word association task was calculated (the Shapiro-Wilk Test indicated that the scores were normally distributed). The result shows that there was a moderate positive correlation $(r=.330)$ between year of learning TCM and scores of the word association task. Nonetheless, the correlation was not statistically significant $(p=.322)$, which is not so surprising given the small number of participants.

A second factor that may have accounted for the differences is whether the TCM learners had extensive English training at university prior to studying TCM. Table 6.6 presents the descriptive results of English trained and non-English trained subgroups of students. As can be seen in Table 6.6, the mean score of the English trained subgroup was much higher $(M=89.83)$ than that of the non-English trained subgroup $(M=55.60)$. The MannWhitney Test was used to explore whether the difference of scores was significant between those two subgroups. The result shows that there was a significant difference $(\mathrm{z}$ $=1.00, p<0.01$ ) between the two subgroups of TCM students even though the sample was small. To put it in another way, those TCM learners who did not have intensive English training prior to TCM study tended to encounter greater difficulty in 
understanding technical words in English than those who had intensive English training. This means general English proficiency mattered in the understanding of TCM technical vocabulary.

Table 6.6: Descriptive statistics of English trained and non-English trained subgroups

\begin{tabular}{|l|l|l|l|}
\hline Subgroup & N & M & SD \\
\hline English trained & 6 & 89.83 & 10.76 \\
\hline Non-English trained & 5 & 55.60 & 13.09 \\
\hline
\end{tabular}

(Note: $\mathrm{N}$ = number; $\mathrm{M}=$ mean; $\mathrm{SD}$ = standard deviation)

Taken together, the results indicate that Chinese TCM learners did have some difficulty in understanding the TCM technical words in the task. Comparatively speaking, more difficulty came from technical words of the lower-frequency levels. For instance, items such as lumbar and diarrhoea were of great difficulty to those learners as the cue words in the task. This pattern was also observed when the learners were confronted with such technical words provided as options to the cue words in the task. The learners did not know technical words such as wheezing, colorectal, and borborygmus. As a result, they did not choose those words as the associations, as was revealed in the interview. Further, the results reveal that whether the learners had English training prior to undertaking the TCM study is an important factor which contributes to better understanding of the TCM technical terms in English.

\subsubsection{Performance of Western TCM learners in the word association task}

Table 6.7 displays the descriptive statistics of the word association task from the Western background TCM learners. For the Western background TCM students as a whole, the mean score of the word association task was 94.20 out of 100 . This score means that the Western TCM learners had good understanding of the target technical words in the task. Nonetheless, it also means that those learners did encounter difficulty in understanding, on average, one word out of twenty target words. 
Table 6.7: Descriptive statistics of the word association task $(\mathrm{N}=10)$ from the Western learners

\begin{tabular}{|l|l|l|l|l|l|}
\hline Category & Total & Minimum & Maximum & M & SD \\
\hline $\begin{array}{l}\text { Frequent and opaque } \\
\text { technical words }\end{array}$ & 25 & 17 & 25 & 22.90 & 2.73 \\
\hline $\begin{array}{l}\text { Frequent and transparent } \\
\text { technical words }\end{array}$ & 25 & 23 & 25 & 24.50 & 0.85 \\
\hline $\begin{array}{l}\text { Infrequent and transparent } \\
\text { technical words }\end{array}$ & 25 & 24 & 25 & 24.90 & 0.32 \\
\hline $\begin{array}{l}\text { Infrequent and opaque } \\
\text { technical words }\end{array}$ & 25 & 19 & 25 & 21.40 & 2.55 \\
\hline All 4 categories & 100 & 86 & 99 & 94.20 & 4.13 \\
\hline
\end{tabular}

(Note: Total= maximum possible score; $\mathrm{M}=$ mean; $\mathrm{SD}=$ standard deviation)

Comparatively speaking, the Western TCM learners scored slightly lower on opaque technical words either from the high frequency band such as mind and blood $(\mathrm{M}=22.90)$ or from lower frequency bands such as tang and phlegm $(\mathrm{M}=21.40)$ than the transparent technical words with mean scores of 24.50 (e.g., knee, cancer) and 24.90 (e.g., lumbar, tonify) respectively (see Table 6.7). The results indicate that the major difficulty of Western background learners came from the opaque words in the understanding of TCM technical words, especially the loan words. Such difficulty was not only reflected in the cue items of the task, but could also be seen from their encoding process when they met loan words provided as options. Two of the participants even made it explicit in the interview that they found it especially challenging to learn words which were borrowed intact from Chinese. In addition, it is interesting to find out in the interview that some low-frequency technical words, such as colorectal and borborygums, were also difficult for the Western learners. Extract 1 illustrates this point. Colorectal was not among the cue words in the word association task, and so this difficulty was not reflected in the scores directly, but was revealed through the interview.

\section{Extract 1: A Western learner talks about associations of the cue word cancer.}

NP6: (Looking at colorectal) What is this word? Colorectal? I don't know this word. 
Interviewer: So you've never seen this word?

NP6: No.

Even though there were only minor differences in scores of the Western background learners, the correlation between year of studying TCM and score of the task was also tested to see if year of studying TCM contributed to the difference. Since the Shapiro-Wilk Test indicated that the scores were normally distributed, the Pearson Correlation $r$ was used. The result shows that there was a weak negative correlation $(r=-.228)$ between year of studying TCM and score of the word association task though it was not statistically significant $(p=.263)$. This result indicates that the year of studying the subject was not an important predictor of the learners' technical vocabulary knowledge. Yet, this result might not be very meaningful because there were other individual differences that mattered with such a small sample. For example, the influence of the parents' cultural background and the learners' ethnicity. As mentioned in Section 6.2.1, two participants came from migrant families with a Chinese background (one at year two and the other one at year three). These two participants performed really well, including on the loan words, and both obtained an overall score of 99 out of 100 .

To sum up, the Western TCM learners in the present study obtained high scores on the word association task even though some of them had certain difficulty in understanding the opaque technical words, especially the loan words. This result indicates that those Western learners seemed to have very good understanding of the technical words in their field of study.

\subsubsection{Comparison of typical associations between Chinese and Western learners}

The second research question concerns whether the Western TCM learners and Chinese TCM learners understand the technical words of their discipline in the same way. In answer to this question, the typical associations trigged by the target technical words of each participant group were calculated and compared. If the typical associations of the cue words were different between the two participant groups, divergence in the understanding of the words was assumed to be manifested. 
Table 6.8: Summary of divergence in each category of TCM technical words

\begin{tabular}{llll}
\hline Degree of divergence & $\begin{array}{l}\text { Major } \\
\text { divergence }\end{array}$ & $\begin{array}{l}\text { Minor } \\
\text { divergence }\end{array}$ & $\begin{array}{l}\text { No } \\
\text { divergence }\end{array}$ \\
\hline Frequent and opaque technical words & 0 & 2 & 3 \\
Frequent and transparent technical words & 0 & 3 & 2 \\
Infrequent and transparent technical words & 0 & 4 & 1 \\
Infrequent and opaque technical words & 2 & 1 & 2 \\
\hline Total & 2 & 10 & 8 \\
\hline
\end{tabular}

As shown in Table 6.8, it was found that major divergence of understanding was manifested concerning two target technical words (10\%) with only two same typical associations in the two participant groups. These items were phlegm and tang. It is not surprising that both the items are from the low frequency and opaque category of technical words. Minor divergence was manifested in ten items (50\%) where both participant groups shared at least three typical associations with these items. It is interesting to note that a minor divergence was manifested from all four categories as indicated in Table 6.8. For the remaining eight cue words, Chinese and Western TCM learners shared the same typical associations, which accounted for $40 \%$ of the lexical items in the word association task.

In sum, divergence in the understanding of technical words from Chinese and Western TCM learners was manifested as a result of divergence identified in the typical associations selected by each participant group. The divergence, however, was minor overall. Such divergence could come from technical words in any category of the Frequency-Transparency Framework with technical words from the lower frequency bands manifesting more divergence than those from the high frequency band. Factors that accounted for the divergence are reported in the next subsection.

\subsubsection{Factors contributing to the divergence of typical associations}

Since divergence was identified in the understanding of the technical words between the Chinese and Western TCM learners, the interview data was analyzed to uncover possible factors that might account for it. Three factors were found in the qualitative analysis as described in Section 6.2.5, which were linguistic background, the breadth of subject 
knowledge, and the cultural background of the learners. The three factors are reported in depth in the following parts in turn.

\subsubsection{Linguistic background}

Linguistic background was identified as the first and most important factor which contributed to the divergence. First, the Chinese TCM learners did not have sufficient knowledge of technical words from the lower frequency bands. That is, the words that they had few chances to meet in their English language learning experiences. For example, borborygmus was chosen by the Western TCM learners as one of the typical associations to the cue word diarrhoea, whereas a majority of Chinese learners (10 out 11) did not select it because the Chinese TCM learners were not at all familiar with borborygmus, which is a technical word in TCM from the lower frequency bands. As a consequence, divergence of associations were manifested. Extract 2 provides an example of which the Chinese learners did not choose borborygmus as the association because she did not know this word.

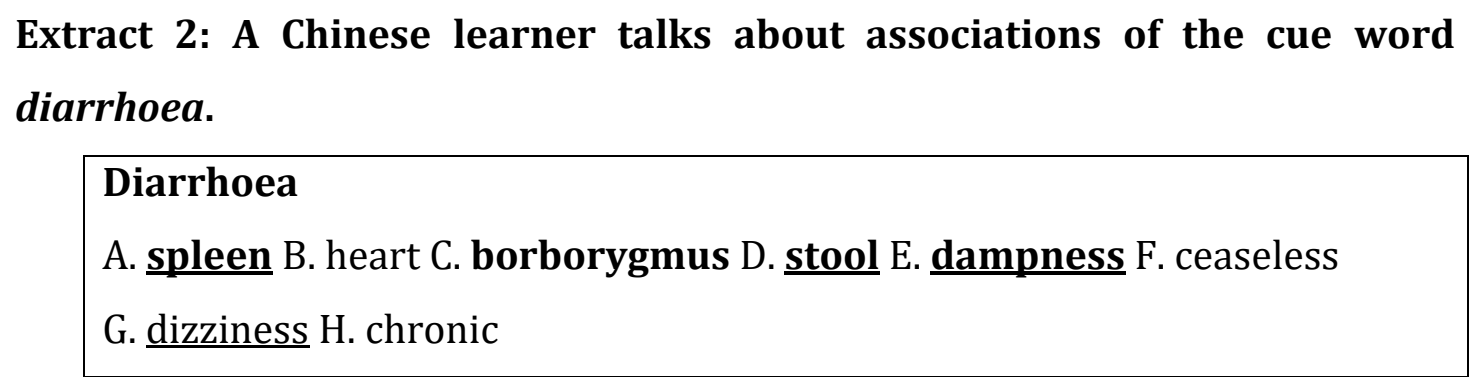

Note: Western learners' typical associations in bold and Chinese learners' underlined.

Interviewer: So can I please ask why you didn't choose C (borborygmus)?

CP10: I don't know this word.

Interviewer: So if you had known this word means fuming (Chinese translation of borborygums), would you have chosen it?

CP10: Fuming, ah, I would.

Interviewer: Why?

CP10: I would remove dizziness, because it has stronger association. This disease has a symptom as such. 
Similar patterns could also be found when the participants met other technical words from the lower frequency bands such as lumbar as the cue word and mucus as the association option. Thus, unfamiliarity with technical words from the lower frequency vocabulary bands affected Chinese TCM learners' choices of associations, which led to the divergence in their understanding of technical words compared to that of Western learners.

Second, the Western TCM learners did not have enough knowledge of the loan words borrowed from Chinese language such as tang as one of the cue words and chengqi as one of the association options. This can also lead to the divergence in the understanding of technical words between Chinese and Western learners. The following extract from an interview with a Western TCM learner illustrates this point.

\author{
Extract 3: Western learner talks about associations to the cue word tang. \\ NP8: (looking at tang) Sounds very hard? \\ Interviewer: Have you ever seen this word? \\ NP8: Oh, no, tang, is that tong means pain? \\ Interviewer: No, not this one. \\ NP8: No, I don't know.
}

Seven out of the ten Western learners showed a similar reaction when they saw this cue word. As a result, a major divergence in understanding of tang was manifested between Western learners and that of the Chinese learners. Thus, lack of Chinese linguistic knowledge on the Western learners' side was an important factor which led to an apparent divergence of understanding certain technical words.

\title{
6.3.3.2 Breadth of subject knowledge
}

The second factor that accounted for the divergence was the breadth of subject knowledge that the learners are familiar with or exposed to in their study. Extract 4 demonstrates one Western TCM learner talking about herbs in relation to cold, whereas Extract 5 illustrates one Chinese TCM learners talking about the same thing. 
Extract 4: One Western learner talking about herbs as association to cold.

Cold

A. fever B. heat C. yin D. pathogenic E. wind F. herbs G. intestine H. limbs

Note: Western learners' (NP3) associations in bold and Chinese learners' (CP12) underlined.

Interviewer: Not herbs?

NP3: I didn't pick herbs because I don't really know anything about herbs yet, and they feel more like a treatment than a problem, yeah.

\section{Extract 5: One Chinese learner talking about herbs as association to cold.}

Interviewer: So why did you choose herbs?

CP12: I chose herbs because a lot of herbs are cold herbs, I mean, some herbs have a cold property.

Even though neither the Western learner in Extract 4 nor the Chinese learner in Extract 5 specialized in herbal medicine, the Chinese learner could still make the association confidently while the Western learner seemed to be less confident when discussing associations about herbs which were outside their specialization. As a consequence, a divergence between the two participant groups' understanding of technical words was manifested. This is because basic courses about TCM foundation, acupuncture and herbal medicine are compulsory in Chinese learners' study irrespective of their sub-disciplines. Therefore, Chinese TCM learners tended to select associations from every aspect of TCM knowledge - TCM theory, acupuncture, and Chinese herbal medicine. In contrast, Western TCM learners tended to have focused study of acupuncture in classes so that they had very focused knowledge of acupuncture and TCM theory as the foundation of their study. As a result, their associations tended to relate more closely to acupuncture either theoretical or practical and TCM theory.

\subsubsection{Cultural background}

Cultural background was assumed to be a factor which accounted for the divergence in understanding of technical words between Chinese and Western background learners. However, it did not actually stood out. In general, Western TCM learners seemed to have a good understanding of most technical words that contain cultural metaphors (e.g., mind, 
phlegm) though it was found that cultural background could create problems in the interpretation of some loan words. In other words, even though TCM has different culturally-bound metaphoric conceptions of the body and matters of health and wellbeing from its Western counterparts, the Western background TCM learners showed a good and similar comprehension of technical words which contain such metaphoric conceptions as the Chinese learners. Such good understanding can probably be ascribed to the good grasp of subject knowledge, which incorporates most of the cultural knowledge the learners needed to understand the technical words in their study. The good understanding of technical words which reflect Chinese metaphoric conception of body and health also implies that the Western background learners have really learned the technical words in their field.

Nonetheless, among the Western background learners, if they had some knowledge about Chinese cultural background, they showed a better ability to decode technical words such as loan words (that are used in the sub-discipline outside their specialization). Extract 6 and Extract 7 compare the decoding process of one Western learner who did not have any Chinese background with another Western learner who had some knowledge of Chinese culture.

\section{Extract 6: Western learner without Chinese background talks about the cue word tang. \\ NP9: Um, tang, that's a tricky one, I don't really know. \\ Interviewer: You haven't seen this word before? \\ NP9: No, well, in like yintang, that kind of thing, but I couldn't tell you...Ok, I will pick none because I can't really, but yeah, I've heard yintang and that kind of thing, but I can't think of any of these that would fit why.}

\section{Extract 7: Western learner with Chinese background talks about the cue word tang.}

NP5: Oh, it's a tricky one coz I actually don't know the meaning I think it's herbs like you know when you have a formula unless I'm completely wrong, yeah, but I actually don't know the meaning of that. Um, also don't even know the meaning of this one (chengqi), so therefore if I think it's closely associated with herbs and I 
translated it into decoction, yeah, I tick that. And then with herbs you can have specific flavours, I'll tick bitter, and them because usually you'll smell them you know, I'll tick that (pungent odour). Interviewer: So how would you translate it if you don't know...?

NP5: Can you pronounce it for me or you're not allowed to?

Interviewer: Tang (the interviewer pronounces it with the tone).

NP5: I think possibly this (decoction), but other than this I don't know.

It shows that when both of the Western learners did not have much knowledge about Chinese linguistic knowledge, the one who had some Chinese background showed a better ability to decode the technical words that were not used very commonly in their specialization but used very commonly in herbal medicine, which is another important area of TCM. The same pattern appeared in other Western learners' decoding process except one who had studied herbal medicine in her previous study. With a majority of Western learners having no Chinese background, major divergence between Chinese and Western learners' understanding was manifested when such technical words acted as a cue word in the word association task, especially loan words such as tang. It is even more interesting to note that some Western learners decoded this word as an English word rather than a loan word. Extract 8 is a case in point.

\section{Extract 8: A Western learner encoded tang as an English word.}

NP1: Ok, tang, I'm not familiar with this word. Is this an English word or Chinese word?

I: Actually it's pinyin.

NP1: I don't know what it is as a Chinese. So I can't make associations. Whereas if I choose to look at it as an English word, then I might be able to make associations. I: So how would you choose as an English word?

NP1: As a taste like a flavour.

As mentioned in Section 5.5.1, tang is a mid-frequency word in English as indicated by Nation's (2012) BNC/COCA base word lists whereas it is also a word form used in Chinese when romanized. Extract 8 thus demonstrates that such loan words were likely to create confusion for the learners if they were not explained explicitly. 
Taken together, it was found that the linguistic background of the learners and the breadth of their subject knowledge appeared to play important roles in the divergence between the two participant groups' understanding of technical words. In addition, cultural background of the learners seemed to matter in interpretation of some loan words. Nonetheless, it seems that the effect of cultural background played only a minor role, which was different from what was hypothesized at the beginning of this study.

\subsubsection{Divergence in understanding of technical words between Chinese and Western learners with same typical associations}

Apart from the findings related to the research questions of this study, one other interesting finding emerged from the results. That is, even though the typical associations were the same, the pathways that led respondents to make certain associations were still different in some cases. Extract 9 and Extract 10 make a comparison of the same typical association to the cue word spleen between a Western learner and a Chinese learner.

\footnotetext{
Extract 9: Western TCM learner talked about lung as association to spleen. NP1: I chose lung because in TCM you got your 12 primary channels, but they are paired up so there is taiyin, so the lung and the spleen are the same channel, and they have a really strong relationship, and they work together quite simultaneously in the body to, basically in creating qi and to ensuring smooth digestion, they both work together particularly in transforming, transporting and moving fluids around the body.
}

\section{Extract 10: Chinese TCM learner talked about lung as association to spleen.}

CP4: Um, because, the spleen and the lung has mother-child relationship. Then digestion, spleen governs transformation and transportation. Then, stomach has an external-internal relationship (with the spleen). Then blood, it's the material basis for production of qi and blood.

As can be seen from the above extracts, the pathways that led the Western TCM learners to make certain association was strongly connected to the knowledge of acupuncture that they had gained from their studies, whereas the Chinese learners came up with the same 
association via the underlying theory and philosophy knowledge of TCM. Such difference actually reveals that the nature of knowledge was determined by the way they learned or acquired that knowledge. The Chinese learners probably acquired much of the underlying theory and philosophy knowledge incidentally in daily life as common sense knowledge, before this knowledge became enhanced and systematized in their study. Therefore, the theory and philosophy knowledge stood out when they made the associations. However, the Western learners spent more time on learning knowledge of their particular sub-discipline even though they also spent some time learning the underlying theory and philosophy knowledge. It is thus not surprising that the technical knowledge of their sub-discipline stood out. This finding suggests that the way the knowledge is represented in the mind is determined by how it was acquired. In Chapter 7, this finding will be discussed in detail to demonstrate the nature of knowledge acquisition (Section 7.3.2).

\subsection{Summary}

The first research question is whether the TCM learners have difficulty in learning and understanding technical vocabulary in their study. This study has found that the Chinese TCM learners had great difficulty in understanding the technical words from the lowerfrequency levels outside the first to the third 1,000 word families of BNC/COCA word lists. Also, some learners had difficulty in understanding the specialized meaning of technical words which was different from their general meaning. In addition, it was found that those TCM learners who did not have intensive English training at university prior to undertaking TCM study encountered greater difficulty than their counterparts who had intensive English training. As for the Western TCM learners, the results showed that they also encountered difficulty in understanding the technical words, but the difficulty mainly originated from the loan words of Chinese origin. The reasons for why Chinese and Western learners met difficulties will be discussed in Section 7.3.1.

The second research question concerns whether Western background TCM learners understand technical words in TCM in the same way as Chinese background TCM learners. It was found that there was a certain divergence of understanding. However, that is mainly because of the different linguistic backgrounds of each participant group. It was also revealed that the learners who had Chinese background tended to understand the 
loan words of Chinese better whereas the Western learners understood technical words of Latin/Greek origin better. The second factor that helped to account for it was breadth of subject knowledge which resulted from the different education systems. The overall conclusion, though, is that the cultural background did not create much divergence in the understanding of technical words as was hypothesized at the beginning of this study. Linguistic knowledge, especially vocabulary knowledge, was a more outstanding factor which led to Chinese and Western background learners' divergence in the understanding of technical words than the cultural knowledge. This is mainly because Western background learners had acquired sufficient cultural knowledge through their courses. In Chapter 7, these findings will be drawn together with other findings for the discussion of factors that make the technical vocabulary difficult for them (Section 7.3.1).

An additional finding of this study is that even though the typical associations were the same in the two participant groups, the pathways that led them to make certain associations were still different in some cases because they learned or acquired the subject knowledge in different ways. It reveals the knowledge that is represented in the mind was determined by how it was learned. This finding will be used in Section 7.3.2 to discuss ways of which the learners from different backgrounds acquired technical knowledge. In the next chapter, the findings of this study along with the findings of the first two studies will be discussed together. 


\section{Chapter 7 Discussion}

\subsection{Introduction}

Chapters 4, 5, and 6 have presented detailed description of three linked studies with answers to the research questions as well as important findings of each study. In this chapter, these findings are discussed in depth under four themes: (1) the importance of technical vocabulary; (2) learners' understanding of technical words; (3) disciplinespecific word list development; and (4) the relation of TCM vocabulary to general vocabulary. The first theme is central to the present thesis while the second theme is an underexplored but potential area of research in technical vocabulary. Both the first and second theme lay foundation to discipline-specific word list construction, which is the third theme of the present chapter. The last theme concerns TCM vocabulary in the broader context of vocabulary learning and teaching, which is important to the understanding of TCM vocabulary and more broadly ESP vocabulary. These themes will be discussed in turn in this chapter.

\subsection{What is the value of technical vocabulary in TCM texts?}

Technical vocabulary is one core concept in ESP vocabulary research. The importance of technical vocabulary and the challenge of learning this kind of vocabulary is clearly demonstrated through the present thesis. Based on the findings of the present research, this section will look at technical vocabulary from two perspectives: (1) highlighting the importance of technical vocabulary from a lexical coverage perspective; and (2) revealing the value of technical vocabulary from a subject knowledge construction perspective.

\subsubsection{How much coverage does technical vocabulary provide?}

Recent studies have greatly advanced our understanding towards the role that technical vocabulary plays in specialized texts. Although different researchers have defined technical vocabulary in slightly different ways or used different approaches to identify technical vocabulary, they have shown that technical vocabulary accounts for a large proportion of technical texts (Chung \& Nation, 2003; Coxhead, 2018; Quero, 2015). This thesis reinforces this idea by revealing that that technical vocabulary covers $39.43 \%$ of the TCM texts. This means that almost two in every five tokens in the TCM texts are technical in nature. 
Earlier research on medicine-related disciplines reported a similar coverage. The text coverage of technical vocabulary is $31.2 \%$ in anatomy (Chung \& Nation, 2003), $37 \%$ in general medical science (Quero, 2015), and 26.73\% with 570 word families in pharmacology (Fraser, 2013). Comparatively speaking, technical vocabulary makes up a much smaller proportion of texts in non-medical disciplines, such as applied linguistics (20.6\%) (Chung \& Nation, 2003). The reason why medicine-related disciplines have a higher proportion of technical vocabulary is because medical disciplines belong to the disciplines of discovering, identifying, and describing new entities or concepts that are not discussed in everyday conversation; thus, they use a large number of highly technical words (e.g., arthrocentesis, olecranarthropathy) (Biber, 2006). In contrast, applied linguistics belongs to the humanity and social science disciplines which discuss new perspectives of people, events and social behavior from everyday life; therefore, many of the technical concepts in humanity and social science disciplines (e.g., language, interaction) can be explained by adapting everyday language (Biber, 2006).

A possible reason why technical vocabulary in TCM provides a slightly higher coverage compared with other medical-related disciplines might be that TCM not only uses a huge number of highly technical words from Latin and/or Greek origin but also a considerable number of loan words to describe concepts that are specifically used in TCM due to its relationship to Chinese culture and philosophy. Excerpt 1 illustrates the use of loan words which are underlined.

\section{Excerpt 1}

Qi is Yang in relation to Blood. Blood, which is a denser and more material form of Qi, is therefore more Yin.

(Maciocia, 2005)

This example shows that it is understandable why technical vocabulary makes up a larger proportion of the TCM texts than in other medical disciplines. In the next subsection, the importance of different types of technical vocabulary in subject knowledge building will be further discussed. 


\subsubsection{How important is TCM technical vocabulary in subject knowledge construction?}

Through the investigation of technical vocabulary of both single words and multiword units, the findings suggest that three types of technical vocabulary are worth noticing but tend to be under researched overall. They are technical words from general highfrequency vocabulary bands, fully technical words, and technical lexical bundles. The importance of these three different types of technical vocabulary will be discussed in turn in this section.

\subsubsection{The importance of technical words from general high-frequency bands}

This thesis first draws attention to the extensive use of general high-frequency vocabulary as technical words. Study 2 found that 1,325 items (47.7\%) of the TCM technical word list $(2,778$ in total) were from the general high-frequency word lists. Earlier studies on technical vocabulary in other disciplines have also reported a similar pattern. Fraser (2009) found that pharmacology employed a considerable number of general high-frequency words as technical vocabulary. Likewise, general high-frequency vocabulary has been found to be frequently used to lexicalize the technical knowledge in law (Tiersma, 1999), in medicine (Hsu, 2013; Quero \& Coxhead, 2018), in engineering (Watson Todd, 2017) and in plastics (Pueyo \& Val, 1996).

More importantly, this thesis advances the research of technical vocabulary, especially TCM vocabulary, by finding out that general high-frequency vocabulary can be used to create the technical knowledge via metaphorization. The metaphorically-based technical words constitute an important area of exploration and explanation. In TCM, for example, the meaning of wind has been extended to denote technical knowledge of disease factors, whereas the meaning of channels has been transferred to denote areas of influence over a certain tridimensinal section of the body (Maciocia, 2005). This is understandable because TCM adopts a holistic approach to describing both health and diseases on the basis of the philosophy of Yin-Yang balance (Kaptchuk, 2001), which advocates the idea that human being is "an integral part of nature" (Yu, 2003, p. 18). Similar use of metaphors at the lexical level was also identified in the discipline of economics (Boers, 1999; Boers \& Demecheleer, 1997; Charteris-Black, 2000), finance (Charteris-Black \& Musolff, 2003), and medicine (Lindstromberg, 1991; Salager-Meyer, 1990; Van Rijn-van Tongeren, 1997). Charteris-Black (2000) reveals that economics can often be 
metaphorically conceived as living organisms. This is instantiated by the metaphorical use of general high-frequency words such as diet in the sentence "State-owned firms surviving on a diet of subsidy and protection" (Charteris-Black, 2000, p. 157). Metaphorical use of general vocabulary can thus be regarded as a way that the writers of discipline specific texts attempt to make the core concepts of their field accessible to outsiders or newcomers (Cameron, 2003). Alternatively, it is possible that metaphors are simply what writers need to resort to when they try to think and talk about intangible or novel concepts (Lakoff \& Johnson, 1980; Van Rijn-van Tongeren, 1997).

In addition, this thesis illustrates some general high-frequency words take on a technical meaning (e.g., expression, labour) in the technical areas which is different from its general meaning. This is an important way in which the general high-frequency vocabulary lexicalizes the subject knowledge in specialized areas such as law (Tiersma, 1999), medicine (Hsu, 2014a), pharmacology (Fraser, 2009), anatomy and applied linguistics (Chung \& Nation, 2003), and biology, history and political science (Cohen, Glasman, Rosenbaum-Cohen, Ferrara, \& Fine, 1979).

Lastly, some general high-frequency words were used equally in everyday experience and in the technical fields (Chung \& Nation, 2003; Hsu, 2013) because each discipline overlaps with our every experience to some extent. Medicine, for instance, is a very common everyday conversation topic while it is also an important academic discipline. Therefore, it is understandable that some medical technical vocabulary overlaps with general vocabulary. Nonetheless, what they mean to a layperson is not necessarily the same as what they mean to an expert. For example, protein might only refer to a substance in food such as meat and egg that is an important part of the human diet to laypeople, whereas it means the total nitrogenous material in plant and animal substances and includes many essential biological compounds such as enzymes or antibody to the experts (protein, n.d.).

In brief, by looking at the use of general high-frequency vocabulary in the specialized discipline of TCM, this thesis reveals an important but underexplored use of such vocabulary to lexicalize the technical knowledge - the metaphorical use. This finding is meaningful from the perspective of ESP vocabulary teaching and learning given that no 
study to date in TCM context and only a few studies (e.g., Salager-Meyer, 1990) in general medical contexts have looked at the metaphorical general high-frequency words.

\subsubsection{The importance of fully technical words}

Fully technical words, as defined here, are lexical items used almost exclusively in medical contexts (Fraser, 2013). One kind of fully technical words in TCM is words of Latin and/or Greek origin (e.g., acetylcholine, hypertension), which is in line with that in Western medicine reported by Hsu (2013) and in pharmacology reported by Fraser (2009). Such fully technical words spread across the mid and low-frequency levels of general vocabulary, and also the TCM medical base word list. On the one hand, those words may be challenging for learners to produce because they tend to be long words. On the other hand, many of them have affixes (e.g., hyper-) and word parts more generally (e.g., tension) that contribute transparently to word meaning, and these may facilitate comprehension (provided the learner understands these word parts). Those words are often used to precisely describe the pathological conditions, medical instruments and clinical procedures (Hutton, 2006). Thus, such technical words of Latin/Greek origin are of great value in subject knowledge construction.

Loan words from Chinese, which is the language of TCM's origin, represent another kind of fully technical words in TCM, and they constitute a unique and interesting group of lexis identified in the current research. This distinctive group of words covered $3.29 \%$ of the total tokens in the TCM Corpora whereas they covered $0 \%$ in the Western medicinal corpora (see Chapter 4 for details). This finding confirms that these loan words are a specialized group of words used only in TCM. The reason why loan words were used to lexicalize the subject knowledge is because TCM contains a sizeable number of concepts which have no counterparts in other medical disciplines and have not been lexicalized in English or cannot be precisely lexicalized in English (e.g., yin, yang). As a result, the use of loan words can be seen as a distinctive feature in the specialized discourse of TCM.

In sum, these findings show that fully technical words are essential in constructing subject knowledge. In section 7.4.3, fully technical words will be further discussed from the perspective of discipline-specific word list construction. 


\subsubsection{The value of TCM technical lexical bundles}

So far in this subsection, the discussion has focused on technical vocabulary in the form of single words. In this part, technical multiword units, which are referred to as technical lexical bundles in this study, will be discussed. Technical lexical bundles, as operationalized here, are the most commonly co-occurring sequences of words in the TCM Corpora which also represent distinct semantic units. This is an understudied but potential area of technical vocabulary research. The very few studies on technical lexical bundles have suggested that technical lexical bundles are pervasive in technical texts (Ward, 2007), and they strongly characterize the specialized texts (Ha, 2015; Ward, 2007). The findings of this thesis lend support to these two claims.

To begin with, a total number of 1,137 technical lexical bundles were found to occur frequently in different types of TCM texts. The sheer number of technical lexical bundles indicates that the use of lexical bundles is a salient feature of the TCM texts. Thus, this finding reinforces the idea that lexical bundles are ubiquitous in technical texts (Ward, 2007). Moreover, this thesis also found that the ubiquity of discipline-specific compound nominal phrases (e.g., heat failure) is a salient feature of the TCM texts, where they serve to name complex phenomena, entities or processes under investigation (Ward, 2007). This finding is in line with the findings of Salager (1983), who identified that compound noun phrases are frequently used in medical English. A possible reason for this is because medical language, like any scientific language, is descriptive. Thus, it makes frequent use of concept expressing nouns or nominal phrases (Salager-Meyer, 1990). Those bundles have the ability to construct meaning and build new knowledge upon previous knowledge (Pueyo \& Val, 1996). Most importantly, Cohen et al. (1979) found that such bundles caused difficulties among ESP learners from different academic disciplines (e.g., biology, history, political science).

Furthermore, Study 2 revealed that technical lexical bundles can construct technical knowledge in a way that the individual words cannot. Due to the special origin of TCM, a large number of loan words are used in TCM texts. Nonetheless, some of the frequently occurring loan words borrowed directly from Chinese may not make much sense by themselves (e.g., bai, ling). For example, ling itself occurs very frequently in the TCM Corpora, but the meaning is unclear by itself unless it is combined with other loan words 
such as fu. Fu ling is a commonly used herb medicine. Technical lexical bundles can therefore make some of the single words into meaningful units, and realize the construction of technical knowledge. This finding also supports the claim of Pueyo and Val (1996) that some technical words cannot be learned in isolation. By looking at the role and nature of technical lexical bundles, this thesis provides sound evidence that the use of technical lexical bundles is a vital tool in the creation of technical knowledge in TCM. Based on these findings, it is suggested that a technical lexical bundle list is beneficial and it can supplement the technical word list in the specialized disciplines.

\subsubsection{Summary}

To sum up, this section has discussed the findings related to technical vocabulary in the form of both single and multiword units. It emphasizes that technical vocabulary plays a crucial role in the technical texts and has a field-constructing function. Also, technical vocabulary strongly characterizes TCM texts, which takes various forms rather than singles words that have traditionally been considered 'technical' only (e.g., using fully technical words). Specifically, technical vocabulary can be composed of general highfrequency words and lexical bundles. Even though there are a number of studies about multiword units in academic disciplines (e.g., Biber et al., 2004; Liu, 2012), research which focuses particularly on technical multiword units remains rare. As a result, this thesis calls for more attention to technical multiword units, and suggests that a technical multiword list can be of help to the discipline-specific word lists which are usually made up of single word units.

\subsection{How important is learners' understanding of technical words in ESP?}

Learners' understanding of technical words is the second thread that is important in the present thesis. In this section, the focus will be on learners' understanding of the technical vocabulary in a word list of their discipline, which is an underexplored but potential area of technical vocabulary research. Section 7.3.1 focuses on factors that make a technical word difficult for the learners, whereas Section 7.3.2 looks at the pathways by which the learners acquire technical vocabulary knowledge in their field of study. After that, I will look more closely at the importance of TCM learners' background in their specialized vocabulary development. The last subsection is a brief summary of Section 7.3. 


\subsubsection{What makes a TCM technical word difficult for the learners?}

As reviewed in the literature (see Section 2.6), there are many potential factors that can make a technical lexical item difficult for ESP learners: the semantic features, specificity, and synforms (Fraser, 2012; Laufer, 1997). This subsection discusses the most salient factors that actually created the most comprehension problems among TCM learners from different backgrounds.

\subsubsection{Chinese background TCM learners}

Study 3 indicated that the specificity of a technical word is the most salient factor which caused comprehension problems among Chinese TCM learners. This finding means that the highly specialized words used exclusively in medical or TCM contexts posed greater difficulty to those learners than those used in wider contexts. For example, technical words such as diarrhoea and lumbar are clearly more difficult to Chinese learners than cancer and protein. These findings are understandable. The Chinese background TCM learners were from the EFL context, thus they had few opportunities to encounter English words outside the general high-frequency vocabulary bands in their previous foreign language learning experience. After they entered into their field of study where they had more chances to be exposed to such technical words, they needed to learn the subject knowledge and those technical words in Chinese and English simultaneously. As a result, they still did not have enough time to use the technical words in English as was claimed by one of the Chinese participants that "I don't have time to use those technical words in English after class because I have many courses to attend in one semester". To make it worse, the overwhelmingly large quantity of such technical words in their discipline made the learning of these words even more challenging. Thus, the Chinese learners experienced difficulty in understanding the technical words, especially those outside the general high-frequency vocabulary bands.

Those technical words which are polysemous or metaphoric $(M=19.91)$ did pose more difficulty compared to other technical words whose meanings are transparent $(M=23.64)$ among the technical words from the high-frequency vocabulary bands. The semantic features of a technical word can thus be regarded as the second salient feature which makes a technical word difficult to the Chinese background learners. For example, the learners knew channel in TV channel but not channel in the body used in TCM. The 
learners knew the word form channel in English and knew its disciplinary concept of channel in their L1 and but their command of English is not sufficient for them to make a positive transfer from general meaning to discipline-specific meaning. As a result, certain semantic characteristics of a technical word may make the technical word hard to understand. It indicates that learners need more help with technical words which are polysemous or metaphoric. Watson Todd (2017) also made a similar claim that technical words which have a different meaning in the specialized discipline from the general meaning should be prioritized in classroom.

\subsubsection{Western background TCM learners}

Study 3 also revealed that, as a whole, the Western background TCM learners had the most difficulty in understanding the loan words (e.g., yang, qi). Such loan words are one type of fully technical words as mentioned in Section 7.2.2.2, which are almost used exclusively in TCM. The possible reason for this finding is that a majority of Western background learners are English L1 speakers and most of them did not have any Chinese background, thus they had little linguistic knowledge about the Chinese loan words. Given this, it is not surprising that the Western background TCM learners encountered greatest difficulty when confronted with such loan words.

In contrast, technical words whose use reflects Chinese cultural metaphors (e.g., heart) did not pose as much difficulty to the Western background TCM learners, and their understanding of these words is similar to that of the Chinese background learners. This finding is surprising as it was assumed at the beginning of Study 3 that the Western background learners may understand such technical words differently from the Chinese background learners. Nonetheless, this finding is understandable for two reasons. First, those Western background learners had studied TCM for a considerable period of time. In other words, they had gained a reasonable or even excellent grasp of the technical knowledge and the cultural knowledge underlying the technical knowledge. Second, the Western background learners received English-medium instruction of the subject knowledge. Such instruction might involve a great deal of semantic elaboration, which can effectively facilitate learners' acquisition of the discipline-specific meanings of those technical words. Given that the Western learners are native or native-like speakers of English, they were familiar with the general meaning of those technical words. Therefore, 
it is easy for them to make appropriate transfer from general meaning of these technical words to the metaphorical meaning. This finding is consistent with the view of Woodward-Kron (2008), who pointed out that learning disciplinary knowledge involves understanding, engaging with, and adopting technical terms of that discourse.

\subsubsection{Summary}

In sum, this thesis emphasizes that the specificity of the technical words is the most prominent factor that makes the technical vocabulary difficult for the TCM learners from different linguistic backgrounds. Further, these findings indicate that TCM learners from different backgrounds encounter different challenges in technical vocabulary learning, which is due in large part to their different linguistic backgrounds. It then suggests that the specialized technical words which the ESP learners rarely encounter in their language learning experience should be the priority in the teaching and learning of technical vocabulary. However, these claims are being made on the basis of a study that involved a small number of participants who completed one task, which limits the generalizability of its findings. In addition, even though learners may be familiar with technically used words from the high-frequency vocabulary bands, their awareness needs to be raised if those words are polysemous or metaphoric in their discipline.

\subsubsection{How did TCM learners acquire knowledge of technical vocabulary?}

The results of Study 3 suggested that the Chinese and Western TCM learners acquired the technical words through different pathways although the resulting knowledge looks the same according to their answers in the word association task. The reason for this is because the way that the knowledge is represented in the learners' mind has been determined by how it was acquired, which is essentially different. The Chinese learners acquired much of the underlying TCM theory and philosophy knowledge incidentally in daily life as common sense knowledge, before this knowledge became enhanced and systematized in their study, whereas the Western learners acquired the TCM technical knowledge mainly through textbooks and courses in their study.

This finding supports a broad interpretation of the concept of transfer appropriate processing proposed by Morris, Bransford, and Franks (1977). Transfer appropriate processing suggests that the way you learn something will determine the nature of the resulting knowledge, and so it emphasizes the importance of aligning particular 
acquisition/learning activities to particular goals and purposes (Morris et al., 1977). For example, Morris et al. (1977) found that the participants who were given a semantic orienting task performed better in a semantic recognition test, while the participants who were give the rhyming orienting task performed better in the rhyming recognition test. This finding is meaningful in two ways.

First, it reminds us that the acquisition activities should match the learning goals and purposes in vocabulary teaching and learning. This is especially important for technical vocabulary pedagogy given that some technical words are general high-frequency words that take on new technical meanings in the specialized disciplines (e.g., heat, channel) and some technical words are highly specialized which are unlikely to be known (e.g., rhinitis, pericardium). The former technical words involve the teaching of new technical meaning to known word forms and the latter involves the teaching of new word forms and new meanings at the same time. Thus, this thesis calls for more attention to different teaching interventions for different technical words. Furthermore, if testing is to be involved, the test and the learning activities should be in an appropriate match. Second, it highlights the value of retrospective interviews immediately after the participants completed the task in the exploratory study. In this way, I can not only see what was learned or acquired by the learners in their study but also see how they learned or acquired that particular knowledge. Such process can in turn provide us with insights into finding appropriate ways to help the learners overcome the difficulty that they encounter in their study.

In brief, this section has revealed the value of particular learning/acquisition activities determined relevant to particular pedagogical goals and purposes. It thus indicates the need to teach technical words of known forms and unknown forms differently.

\subsubsection{How important is learners' background in their ESP vocabulary development?} It was revealed in Study 3 that the Chinese background TCM learners had an average score of 74.27 on the word association task, while the corresponding score of the Western background learners is 94.20. Even though the present thesis did not aim to compare the performance of the Western learners and Chinese learners over the word association task, such results are still worth some interpretation and discussion. 
These results indicated that the Chinese background learners did not have as good an understanding of technical words in the given word association task as Western background learners did overall. This finding however is understandable considering the different learning situations of the two groups of learners. For the Chinese learners, they were in English as a foreign language context where Chinese is the medium of instruction in their study. Those Chinese learners were expected to acquire specialized English language ability mostly through specialized English courses. This means they had limited exposure to English-medium textbooks/instruction each week and thus limited time to use technical vocabulary in English. Nonetheless, during the inevitably limited learning time, they had to learn the novel word forms and meanings as a majority of the technical words were new to them. Even if they might be familiar with some technical words from the general high-frequency vocabulary bands and the technical knowledge in their L1, it is still difficult for them to make semantic transfers from general meanings of these technical words to their technical meanings as discussed in Section 7.3.1.1. In contrast, the Western TCM learners lived in an English speaking country and also had it as the medium of instruction in their study. That means two things. First, the Western learners were very likely to be familiar with most of the technical words in form even though there must be some low-frequency words in TCM whose forms are also unfamiliar to native speakers (e.g., loan words of Chinese origin). Thus, the Western learners could focus on learning the technical meaning of the words whose form was known to them. Second, they had more exposure to English-medium materials/instruction and thus more time to be engaged with the technical vocabulary.

This finding is interesting as it is in line with the 'type of processing-resource allocation' (TOPRA) model for vocabulary learning (Barcroft, 2004, 2015). According to the TOPRA model, each specific type of processing necessarily exhausts the overall processing capacities of a learner, and thus decreases other types of processing (Barcroft, 2015). In the case of Chinese TCM learners, they needed to focus on two types of processing - both structural and semantic processing - when learning technical vocabulary during the limited time of specialized English courses. By contrast, the Western learners only needed to focus on semantic processing to a large extent. That is, the Chinese learners had heavier processing demands than the Western learners, which may have led to a relatively lower learning rate of technical words by the Chinese learners than the 
Western learners. As a result, the Chinese learners appeared to have poorer knowledge of the technical words in the word association task than the Western learners.

In sum, the findings of this study emphasize the different processing demands of the learners from different linguistic backgrounds. It also suggests that the learning and teaching of technical words in known form and unknown form should be different.

\subsubsection{Summary}

To sum up, this section reveals that learners of different linguistic backgrounds have different sources of difficulty in technical vocabulary learning. In general, they encountered greater difficulty with technical words that they seldom saw or used in their previous language learning experience. Thus, it suggests that learners' linguistic background should be considered in ESP vocabulary teaching and learning, and in discipline-specific word list development. Further, this thesis emphasizes that the teaching and learning of technical words in known word forms and in unknown word forms should be different as the former only involves semantic processing and the latter involves both structural and semantic processing (Barcroft, 2015).

\subsection{How can discipline-specific word lists better support ESP vocabulary development?}

Discipline-specific word list construction is another core issue of the present thesis. It is thus important to look closely at the factors related to discipline-specific word list construction emerging from the results of this thesis. This section first provides sound evidence why discipline-specific word lists can better serve ESP learners' needs than general lists. After that, it discusses one important issue of discipline-specific word lists - whether technical words from the general high frequency vocabulary bands should be included. Then it also discusses the importance of including fully technical vocabulary in the discipline-specific word lists. Lastly, it looks at the importance of learners' linguistic background and proficiency levels in discipline-specific word list development.

\subsubsection{How can TCM technical word list better serve TCM learners' needs?}

Compared with generic high-frequency or academic word lists, discipline-specific word lists are better at drawing ESP learners' attention to the most important words in their specialized disciplines and providing a shortcut to reduce the amount of vocabulary 
learning given that academic disciplines usually have heavy vocabulary loads (Nation, 2013, 2016a). This thesis provides strong support for this argument in three ways.

First, Study 1 found a large number of frequently used lexical items (e.g., qi, fructus) in TCM did not appear in any of the existing BNC/COCA base word lists, even the lowest frequency level of 25, 000 word families. Nor did they appear in the generic academic word lists such as Coxhead's (2000) AWL and Gardner and Davies' (2013) AVL (Lu \& Durrant, 2017). A similar pattern was found with medical vocabulary as reported in Study 1 (see Section 4.5.5). Pneumoniae and pathobiology are cases in point. These findings are consistent with those from earlier studies. For example, research on vocabulary of environmental science reported that the GSL and AWL did not capture some of the frequently used and widely distributed words in that discipline (Liu \& Han, 2015). The findings of the present thesis and of previous research indicate that some of the very important lexical items in specialized disciplines may not be captured by the general word lists or generic academic word lists. Thus, a discipline-specific word list is needed so that all the important items in the given discipline can be well captured.

Second, Study 1 also found that from the seventh 1,000-word-family level onwards, the additional coverage for each 1,000 level of vocabulary in the TCM Corpora is very small, at under $1 \%$. Most of such coverage was achieved by a small number of frequently occurring items in these base word lists. That is, only a small number of the items in the mid and low-frequency bands are truly useful to the TCM learners. This finding lends support to the idea that a focused discipline-specific word list can better serve ESP learners' specific lexical needs (Durrant, 2013, 2016; Hyland \& Tse, 2007).

Third, this thesis demonstrated that some words take on a technical meaning when used in the specialized disciplines. For example, period in medical context refers to menstruation rather than a period of time. A discipline-specific word list allows the ESP learners and teachers to focus on the discipline-specific meanings of the items in the lists and on how these words are used in the disciplines that the learners are studying. Similar vocabulary use was found in other disciplines such as engineering (Watson Todd, 2017), pharmacology (Fraser, 2009), carpentry (Coxhead et al., 2016), and economics 
(Sutarsyah et al., 1994). These findings thus provide more solid evidence to the idea that discipline-specific word lists can better serve ESP learners' needs.

In brief, this thesis provides sound evidence that the TCM technical word list can better serve TCM learners' needs, which more broadly promotes the idea that discipline-specific word lists can better reflect the specialized features of the specialized fields and better serve the specific needs of the ESP learners from three different perspectives. In this way, the ESP learners can be motivated to learn items from these lists because they can clearly see the relationship between what they learn in their ESP courses and their subject courses (Coxhead \& Hirsh, 2007; Hyland, 2016).

\subsubsection{Should technical words from general high-frequency vocabulary be included in the discipline-specific word lists?}

While several existing discipline-specific word lists (Hsu, 2013; Liu \& Han, 2015; Wang et al., 2008; Yang, 2015) were developed on top of the general high-frequency word lists, the others (Coxhead et al., 2016; Fraser, 2009; Lei \& Liu, 2016; Valipouri \& Nassaji, 2013; Ward, 2009; Watson Todd, 2017) did not rely on any existing word lists. The first approach considers that specialize vocabulary is related to general vocabulary and assumes that the ESP learners have learned and mastered the general high-frequency vocabulary. Nonetheless, the latter approach regards the specialized vocabulary as a separate set of vocabulary cutting across different 1,000-word levels of general vocabulary, and does not assume that the learners have mastered the general highfrequency vocabulary. The findings of the present thesis lend support to the latter approach.

First, as can be seen from its structure, the TCM technical word list has a large proportion $(47.7 \%)$ of shared items with the first to the third 1,000 BNC/COCA word families. This finding means that TCM technical vocabulary has a large number of shared items with the high frequency vocabulary, indicating that general high-frequency vocabulary is an indispensable part of the TCM discipline-specific word list. Quero and Coxhead (2018) also identified a similar vocabulary pattern in medicine, in which they found that around $15 \%$ of first to the third 1,000 BNC/COCA word families have a medical meaning. Second, Study 3 reveals that some TCM learners had not mastered all the general high-frequency 
words in the TCM technical word list. The Chinese TCM learners had an average score of 43.55 (a total of 50) over the technical items from the high-frequency vocabulary bands, whereas the Western learners scored 47.4 on average. These findings indicate that the technical words from high frequency vocabulary are of great pedagogical value. It thus highlights a weakness of the discipline-specific word lists which were built on top of the general high-frequency word lists such as West's (1953) GSL, assuming that the learners had mastered those words.

Given the importance of technical words from general high-frequency bands, this thesis reinforces the idea that technical vocabulary is a separate kind of vocabulary cutting across different frequency levels of general vocabulary (Coxhead et al., 2016; Nation, 2013; Nation et al., 2016). Further, this thesis argues that it is necessary to include technical words from the general high-frequency vocabulary levels in the disciplinespecific word lists to better support the ESP learners' vocabulary development.

\subsubsection{Should fully technical words be included in the discipline-specific word lists?}

A number of previous discipline-specific word lists (e.g., Hsu, 2013; Wang et al., 2008) focused on discipline-specific academic words and did not take fully technical words into consideration for two reasons. First, the researchers claimed that fully technical vocabulary has constancy of meaning unmodified by association and has only one denotation. They are thus more easily mastered than other kinds of vocabulary (Hoffmann, 1981). Second, it was believed that ESP learners do not see these technical words as a problem because these words are usually the focus in the subject courses or the textbooks (Ferguson, 2012; Hsu, 2013; Strevens, 1973).

Nonetheless, the findings of the present thesis suggest that fully technical words should also be included in the discipline-specific word lists. First, they are crucial in lexicalizing the subject knowledge of that field as indicated in Section 7.2.2.2. Second, it was revealed in Study 3 that Chinese learners, who are ESP learners from the EFL context, encountered great difficulty in comprehending fully technical words (e.g., colorectal, borborygmus) even though these words have been taught in some courses for terminology in their discipline. This might be that the learners do not encounter these technical words frequently enough to allow them to acquire those words. According to Nation (2013), 
repeated attention to the target items is required for vocabulary learning. In such contexts, sustained attention to items of fully technical vocabulary has become necessary, which is echoed by Ferguson (2012). He further points out that ESP teachers may well be able to assist with the learning of such technical vocabulary. For example, they can help medical students who have sound knowledge the meaning of technical terms but still struggle over their pronunciation. Additionally, incorporating fully technical words into the discipline-specific word list allows the learners and teachers to systematically focus on the frequently used technical words given that there is a huge amount of technical words in most academic disciplines. As a result, they can sequence the learning and teaching of technical words based on informed decisions. In this way, all technical items that the learners encounter often in their disciplinary areas can be captured and learned systematically.

\subsubsection{How can word lists be adaptable to learners' linguistic backgrounds and proficiency levels?}

Nation et al. (2016) suggests that word lists should suit the proficiency level of the list users. Moreover, Dang et al. (2017) also calls for attention to learners' previous vocabulary knowledge in the development of specialized word lists. The nature of technical vocabulary in TCM revealed in the current thesis provides insight into the value of considering learners' linguistic variation in discipline-specific word list construction. Such consideration is especially important for discipline-specific word lists since the target users can be English L1 speakers, L2 speakers, or EFL learners who are studying the given academic discipline. The TCM technical word list is an example of how a discipline-specific word list can better match its users' linguistic background and proficiency levels by way of dividing the long list into smaller sets of words.

This TCM technical word list was first divided into three sub-lists according to the number of occurrence of the technical words in the TCM Corpora in a decreasing order (see Figure 7.1). Sub-list 1, 2 and 3 represent the most frequent 1,000 word types, the second most frequent 1,000 word types, and the third most frequent 778 word types, in the TCM Corpora respectively. Each sub-list was divided into three levels, with Level 1, 2, and 3 consisting of technical words from high-frequency vocabulary bands, technical 
words outside high-frequency bands, and technical words which are loan words, respectively (see more details in Section 5.3.1).

\section{Figure 7.1: Division of the TCM technical word list}

\begin{tabular}{l|l|l|l} 
Sub-list 1 & Level 1 & Level 2 & Level 3 \\
\hline Sub-list 2 & Level 1 & Level 2 & Level 3 \\
\hline Sub-list 3 & Level 1 & Level 2 & Level 3
\end{tabular}

These levels allow learners to focus their attention on the TCM technical words that are relevant to their existing vocabulary levels. Now, let us look at how the division of the technical words can adapt to the learners of different linguistic backgrounds and proficiency levels.

\subsubsection{The Chinese background TCM learners}

Study 3 showed that some TCM learners had not mastered technical vocabulary from general high-frequency vocabulary bands. Such learners are defined as intermediate vocabulary level learners. These learners can start with Level 1 and then go to Level 2 of each sub-list. Given that the Chinese learners have knowledge of loan words at Level 3, they only need to focus on Level 1 and Level 2 technical words from sub-list 1 to sub-list 3. In this way, learners at intermediate vocabulary level can focus their attention on the most important words that they will encounter in their discipline. At the same time, they can make up for their insufficient knowledge of the general high-frequency vocabulary.

As also shown in Study 3, some Chinese learners have an adequate grasp of technical vocabulary from general high-frequency bands but have not mastered technical vocabulary outside the high-frequency bands. These learners are defined as advanced vocabulary level learners. Ideally, these learners can focus on Level 2 items directly from sub-list 1 to 3 . It allows the learners to focus their attention on a much smaller number of items based on their existing vocabulary level. 


\subsubsection{The Western background learners and other non-Chinese learners}

As revealed in Study 3, the western background TCM learners are either L1 or L2 English speakers, who have good command of the Level 1 and Level 2 technical words. However, they lack knowledge of the loan words at Level 3. In this case, they need a much smaller number of words and can focus their attention on the loan words only.

Accordingly, other non-Chinese learners can also adjust their focus on the levels depending on their vocabulary level and knowledge of loan words. For example, the high vocabulary level learners who do not have linguistic knowledge of Chinese can start from technical words at Level 2 and then Level 3. Ideally, the learners can get the best return from their efforts by studying the TCM technical words beyond their existing vocabulary levels.

Overall, this thesis shows that it is important for list makers to consider the linguistic characteristics of their target users in word list development to make the word list a more efficient tool (Dang et al., 2017; Nation et al., 2016). Another advantage of the division of sub-lists and levels is that it allows us to divide the long technical word lists into smaller and manageable sets of items. Further, the teachers and learners can avoid repeatedly teaching and learning known items.

\subsection{How does TCM vocabulary relate to general English vocabulary?}

The concept of general high, mid, and low-frequency vocabulary runs through the first two studies of the present thesis as the foundation of technical vocabulary research. By analyzing the coverage of BNC/COCA base word lists at each frequency level, this section (1) emphasizes the value of high, mid, and low-frequency vocabulary in specialized TCM texts; and (2) reveals the large vocabulary burden nature of the ESP texts.

\subsubsection{What is the importance of the high, mid, and low-frequency vocabulary in TCM vocabulary learning?}

By revisiting frequency, lexical coverage, acquisition and use, Schmitt and Schmitt (2014) determined that high-frequency English vocabulary should include the most frequent 3,000 word families. This cut-off point is different from the traditional idea that the general high-frequency vocabulary included roughly the most frequency 2,000 word 
families, which was largely influenced by the size of West's (1953) GSL (Nation et al., 2016; Schmitt, 2010b; Schmitt \& Schmitt, 2014).

The results of this thesis lend support to Schmitt and Schmitt's (2014) idea that highfrequency English vocabulary should include the most frequent 3,000 word families from two perspectives. First, the first three 1,000 word families contributed 56.11\%, 10.46\%, and $8.74 \%$ to the coverage of TCM texts. The coverage dropped dramatically to $3.86 \%$ by the fourth 1,000 word families, and even lower on the base word lists after the 4,000 word families. Apart from the coverage figures, the number of word types found in the TCM corpus also support the inclusion of the first 3,000 word families in the highfrequency vocabulary. The first three 1,000 word families involved 4,048, 3,706 and 3,697 word types, whereas the fourth 1,000 word families involved a much smaller number of 2,234 word types. These findings reveal that the third 1,000 word families are no less important than the second 1,000 word families in the TCM texts, but the difference between the third and fourth 1,000 word families is apparent. Thus, this thesis lends support to the idea that the most frequent 3,000 word families are an appropriate boundary for the high-frequency vocabulary. Providing a total coverage of $75.31 \%$, this study reveals that the TCM learners can recognize a large proportion of the word forms in the TCM texts if they have knowledge of the general high-frequency vocabulary. It thus highlights the value of general high-frequency vocabulary in specialized texts, which conforms to previous claims that knowledge of high-frequency vocabulary is crucial for all kinds of language use (Gilner, 2011; Nation, 2001; Nation \& Hwang, 1995). As a result, this study demonstrates that the high-frequency vocabulary indeed deserves focused attention from TCM learners and teachers.

This study then raises the question about the suitability of defining the mid-frequency vocabulary as from the most frequent 4,000 to 8,000 word families (Schmitt \& Schmitt, 2014 ) in the specialized discipline of TCM because of the high coverage of two TCMspecific base word lists. Both TCM base word lists have higher coverage than base word lists from the fifth 1,000 word families onwards as shown in Study 1. Nonetheless, further research in other disciplines is needed to confirm whether it is true in other disciplines. It also casts doubt on the utility of mid-frequency vocabulary as a whole for ESP learners who have highly specialized language needs as in this study. From the seventh 1,000- 
word-family level onwards, the additional coverage for each 1,000 level is very small indeed, at under 1\%. Further, most of this coverage was achieved by a small number of items in these base word lists. That is, only a small number of items in the mid-frequency are truly useful to the TCM learners. The finding thus lends support to the suggestion that a more focused discipline-specific word list can better serve ESP learners' needs (Durrant, 2013; Hyland \& Tse, 2007).

In terms of the low-frequency vocabulary which was considered to have limited utility generally (Schmitt \& Schmitt, 2014), this study holds a different view of their utility to TCM learners. Two reasons may explain this. First, a vocabulary load of at least the most frequent 12,000 word families at 98\% coverage level found in Study 1 means the lowfrequency vocabulary is an indispensable part of the TCM vocabulary. The TCM learners will encounter certain difficulty without knowledge of vocabulary at the low-frequency bands. Second, it was found that some low-frequency items were essential to the construction of subject knowledge (e.g., decoction, pericardium), but only a relatively small number of the items from the low-frequency bands are truly useful, which is the same as vocabulary of mid-frequency bands as mentioned above. Similarly, it promotes the idea that the ESP learners can get better learning return from a more focused discipline-specific word list that is tailored to their specialized needs (Durrant, 2013; Hyland \& Tse, 2007).

In summary, by looking at the lexical profile of TCM vocabulary along the BNC/COCA base word lists, this thesis adds sound evidence to the need of including the most frequent 3,000 word families as high-frequency vocabulary. However, this study challenges the suitability of defining the most frequent 4,000 to 8,000 word families as mid-frequency vocabulary in the specialized discipline of TCM and calls for more attention to the lowfrequency vocabulary. Together, it suggests that the learning sequence after the most frequent 3,000 word families should be a more focused discipline-specific word list to better serve learners' specific language needs.

\subsubsection{What is the value of vocabulary load analysis in understanding TCM texts?}

Study 1 has shown that vocabulary load analysis is an important tool in evaluating the lexical demands of the given ESP texts. To begin with, it allows for fair comparison of 
vocabulary difficulty between different disciplines or genres. In Study 1, it was found that knowledge of the most frequent 13,000 and 7,000 word families (+ proper nouns, marginal words, transparent compounds, abbreviations and two TCM word lists) was needed to reach the $98 \%$ and $95 \%$ coverage in TCM texts. Compared with the vocabulary load of 8,000-9,000 word families (+ proper nouns) to reach $98 \%$ coverage and 4,000 word families (+ proper nouns) to reach 95\% coverage of general written English (Nation, 2006), the findings suggest that a larger vocabulary load was needed to achieve both optimal and minimum comprehension in the discipline of TCM. Further, the TCM textbooks are also more lexically demanding than engineering and business textbooks. The TCM textbooks required knowledge of the most 7,000-8,000 word families to reach 95\% coverage, while the corresponding figures for engineering and business textbooks are 5000 word families (Hsu, 2014a) and 3,000-4,000 word families (Hsu, 2011) respectively. These findings are in line with findings of Dang and Webb (2014), who found that Life and Medical Sciences is the most lexically demanding specialized area in examination of vocabulary size necessary to reach $95 \%$ and $98 \%$ coverage compared to other specialized areas of the same spoken genre (Arts and Humanities, Physical Sciences and Social Sciences). This finding is also broadly consistent with that of Coxhead, Dang, and Mukai (2017), who pointed out that comprehending hard-science speech (e.g., engineering, medical science) is more demanding than comprehending soft-science speech (e.g., law, education) in terms of lexical coverage. It is thus understandable that TCM is a lexically demanding discipline compared to other disciplines of the same written genre.

Second, Study 1 also revealed that there was a slight difference in the amount of vocabulary needed to reach $95 \%$ and $98 \%$ coverage of theory-based textbooks, practicebased textbooks and journal articles. With knowledge of proper nouns, marginal words, transparent compounds, abbreviations and TCM base word lists, learners need 7,000 word families to reach $95 \%$ of the theory-based textbooks and journal articles while they need 8,000 word families for the practice-based textbooks. At the $98 \%$ level, the vocabulary burden for both types of textbooks is 13,000 word families (+ proper nouns, marginal words, transparent compounds, abbreviations and two TCM word lists), while 12,000 for journal articles. It is interesting to note that journal articles are likely to have a slightly lower vocabulary burden than both types of textbooks. 
One possible reason for this small but important discrepancy between lexical demands of TCM textbooks and journal articles is that textbooks are information dense in nature in the sense that each chapter covers a whole set of related medical terms. As pointed out by Biber (2006), textbook writers are motivated by a stylistic preference for varied vocabulary, rather than using the same word types repeatedly. Such diversity in word choices of textbooks is due mostly to the use of specialized vocabulary. In contrast, the journal articles have a tighter focus than textbooks on a particular medical issue, and they are often more homogenous in terms of format, research process and content. Thus, the journal articles are likely to use a less wide variety of word types. Further, journal articles perhaps contain more numerical information, such that a substantial number of tokens are numbers and figures. The difference between the nature of textbook and journal article can be illustrated in the following excerpts which were taken from the textbook and journal article corpora in the present study.

\section{Excerpt 2}

Ear acupuncture is a well-known application of the principle that a single, small part of the body reflects the whole. According to this theory, the ear resembles an upside down fetus and there is a point in the ear pavilion that reflects each part or organ of the body (Fig. 23.3).

(TCM textbook corpus)

\section{Excerpt 3}

A total of 458 subjects were assessed for eligibility and 71 subjects were enrolled, 39 were randomised to the intervention group and 32 to the placebo group. Eight randomly allocated participants did not complete the study; four in each group (see Figure 1).

(TCM journal article corpus)

Excerpt 2 is from the textbooks which introduces a treatment method in TCM, while Excerpt 3 is from a journal article which reports a research study. It can be seen from these excerpts that textbooks use a set of related terms while the journal articles uses considerable numbers to report the results of a research study. As a result, it is 
understandable that the TCM journal articles are less lexically demanding than the textbooks.

These findings are in contrast to research by Hsu (2011), who reported that business journal articles had a heavier vocabulary burden than business textbooks. One explanation for this contrast might be that different disciplines have different practices and routines (Biber, 2006). Medical students usually need to learn a huge number of highly specialized technical words to understand the subject knowledge to enter this field. However, what is probably unusual for medical learners is that they need to learn to describe technical terms in a colloquial way at the later stage of study in order to communicate with patients who are usually not professionals (Wette \& Hawken, 2016). The patients might not have any knowledge of medical terminology, yet the ways in which they describe their histories and talk about their symptoms (using informal, lay-medical language) are of vital importance to the medical professionals' ability to understand what is going on with the patients and to reach a provisional diagnosis. Miscommunication may occur in consultations if the doctors misinterpret the lay medical language used by the patients (Dahm, 2011). In fact, one important component of clinical communicative skills is to use commonplace synonyms for medical jargon and terms (Dahm, 2011; Wette \& Hawken, 2016). Textbooks can be regarded as tools which help medical students enter this field, whereas research articles reflect mature medical students or professionals' way of reporting research. Thus, research articles in medical contexts tend to use more colloquial technical words compared to textbooks so that they can be better understood. Both findings are meaningful in raising the point that textbooks alone or journal articles alone might not be sufficient to represent the lexical components of a discipline. Including both textbooks and journal articles is the strength of the corpora used in the present study.

In sum, this section has discussed the findings related to lexical profile and vocabulary loads of TCM. It demonstrates that vocabulary load analysis is an important tool in the evaluation and understanding of vocabulary in specialized disciplines. As a result, it highlights the importance of well-constructed frequency-based BNC/COCA word lists as a foundation for the vocabulary load analysis. Also, it indicates that TCM is a lexically 
demanding discipline. Thus, it warrants that a focused TCM-specific word list will be of help to support the ESP vocabulary learning and teaching.

\subsection{Summary}

This chapter has discussed four main themes drawn from the findings of the present thesis. Technical vocabulary and discipline-specific word list construction are core issues in ESP vocabulary research. Learners' understanding of the technical vocabulary is an understudied but potential area of technical vocabulary. The last theme of the relationship between ESP vocabulary and general vocabulary is of fundamental importance in ESP vocabulary research. The next chapter concludes this thesis by looking at the implications of the thesis as well as its limitations. It will also point out directions for future research. 


\section{Chapter 8 Conclusion}

\subsection{Introduction}

This thesis has explored the nature of vocabulary in Traditional Chinese Medicine (TCM) as an important academic discipline to address the lack of research on specialized vocabulary in this area. The first study investigated the lexical profile of TCM through corpus analysis and measured the vocabulary burden of TCM textbooks and journal articles. It mainly addressed the question of whether, and how, a pedagogically useful listing of technical words can be constructed. Based on this analysis of vocabulary difficulty and richness, the second study involved establishing a 2,778-item TCM technical word list supplemented with a 1,543-item TCM lexical bundle list for the learners and teachers in this specialized but underexplored discipline. This study has answered the questions concerning what words the TCM learners actually need to know for successful academic communication in their target discourse community. The last study is an exploratory study, which served a diagnostic purpose to identify problematic technical words from the learners' perspective using items in the TCM technical word list. This exploratory study started with an assumption that the Western background learners and Chinese background learners in TCM might understand technical words with cultural metaphors in different ways. However, the findings of the present research show that this assumption does not hold. Such findings are surprising but interesting. The Western background TCM learners understood the technical words with cultural metaphor in much the same way as the Chinese learners since the Western background learners obtain an excellent grasp of subject knowledge in their field of study. However, certain divergence was manifested in the understanding of the technical words between the Western and Chinese learners, which mainly originated from the difference of their linguistic knowledge. These three linked studies provide valuable implications for theory, methodology, teaching, and learning of vocabulary especially technical vocabulary.

Chapter 8 starts with the theoretical and methodological contributions of the present thesis (Section 8.2). It is followed by the pedagogical implications (Section 8.3), and limitations (Section 8.4). This chapter ends with the directions for future research and a brief conclusion of the present thesis (Sections 8.5 and 8.6). 


\subsection{Methodological and theoretical contributions}

This section is devoted to discussing the methodological and theoretical contributions of the current research. These contributions are discussed in turn below.

\subsubsection{Methodological contributions}

The present thesis has made three methodological contributions in relation to corpus design, the identification of technical vocabulary, and the value of interviews with the word association task.

The first methodological contribution is the way in which the textbook corpora were built to represent textbooks used at two different stages of medical education. This was determined by the nature of medical education in which the TCM learners usually start with the TCM theories and then proceed to clinical practices. Textbooks at these two stages are quite different in focus, and the difficulty level is progressive from the theory to practice-based textbooks. The analysis of theory and practice-based textbooks revealed that the practice-based textbooks have a slightly heavier lexical burden than the theory-based textbooks, which reflects the nature of lexis in textbooks used at these two different stages. In this way, the textbooks that are encountered by the learners in their study are well represented, and the nature of lexis used in their discipline is well reflected. This way of developing a textbook corpus is quite different from previous medical corpus-based studies which usually draw on different sub-disciplines to build a representative corpus (e.g., Hsu, 2013; Lei \& Liu, 2016). It is sensible to sample the medical corpus on different sub-disciplines. However, in reality, the medical learners do not progress from one sub-discipline to another in their undergraduate study. Instead, they need to learn foundational knowledge and practice in their undergraduate study and then move to a certain sub-discipline which usually happens in the postgraduate study. No previous corpus-based research on medical vocabulary has taken this issue into account. Thus, the approach of corpora design in the current thesis has a methodological value.

Second, this thesis makes a methodological contribution by way of making the TCM technical word list adaptable to learners' linguistic background and proficiency level. The TCM technical word list was developed independently without relying on any generic 
high-frequency word lists. It has been divided into three sub-lists to make it in manageable sets of words, and each sub-list was divided into three different levels to match users' linguistic background and vocabulary levels. This is very important given that the TCM learners are from a variety of linguistic backgrounds as a result of the internationalization of higher education. Even if the learners are from the same linguistic background, it is highly possible to have different vocabulary levels. This can be inferred from the results of the word association task reported in Section 6.3.1. Some Chinese learners only know an average 8 out of 20 items while others might know all of the 20 items. Dividing the lists into different levels enables us to take learners' existing knowledge of general vocabulary into consideration, and thus can avoid repeatedly teaching and learning known items. Such way of making a list adaptable to learners' vocabulary level has been used by Dang et al. (2017) for the Academic Spoken Word List. Yet, this is the first study to extend such idea to a technical word list. In brief, the TCM technical word list presents a way to make a corpus-based word list more suitable to ESP vocabulary learning and teaching.

The third methodological contribution of the thesis is that it highlights the value of combining a word association task and a follow-up interview in the exploratory research to investigate learners' understanding of technical words. A word association task can be a useful tool to reveal learners' knowledge of the given technical words, while interviewing the learners apart from the word association task as an extra dimension can help us to discern what knowledge drives the given association, which cannot be revealed by the outcome of the word association task. Even though the Chinese and Western learners have chosen the same association to a cue word according to the outcome of the word association task, the interview data revealed that the pathways which led the Western and Chinese learners to choose certain association can be very different. Such finding illustrates that a combination of word association task and interview allows us to obtain rich and complementary data in understanding learners' knowledge of the technical words. That is to say, the word association task and the follow-up interview is a nice methodological twist. 


\subsubsection{Theoretical contributions}

The present thesis has also made two theoretical contributions to vocabulary research. First, it provides insights into the nature of the technical vocabulary in an important but underexplored discipline of TCM. This research emphasizes that technical vocabulary in TCM can take different forms, be of different linguistic origins, and also in either single or multiword units. Importantly, this study is one of the first studies that has looked at technical vocabulary from the perspective of multiword units. It reveals that some technical words should be seen in multiword units rather than in single words as only in combinations do they represent a meaningful unit. Drawing on an example from Section 5.5.5, ling occurred frequently in the TCM Corpora, but the meaning is not clear by itself unless it was combined with other words such as fu. Fu ling is a commonly used herb medicine. Thus, this research has illustrated that lexical bundles are useful devices for the construction and comprehension of a specialized discourse. Considering the lack of research into technical vocabulary of multiword units, the findings of this thesis provide important insights for further research about technical vocabulary in different ESP areas.

Second, this thesis provides empirical insights into learners' knowledge of technical vocabulary in their field of study. It indicates that TCM learners tended to lack knowledge of highly specialized technical vocabulary, especially those words which are used exclusively in their discipline. This finding is meaningful given that it is the first research to date which has investigated the actual problems encountered by the learners in technical vocabulary learning. It then provides further insights into how to prioritize the technical items for instruction in a specialized English course given that there are always more items needed to be taught than the actual learning time available, which is the main focus of the next section.

\subsection{Pedagogical implications}

This section addresses pedagogical implications of the present thesis and offers recommendations for ESP vocabulary learning and teaching from four perspectives. Section 8.3.1 offers suggestions about developing learners' knowledge of technical words, while Section 8.3.2 provides suggestions about developing learners' knowledge of technical lexical bundles. After that, Section 8.3.3 is devoted to discussing implications related to learners' understanding of technical words in the word list implementation. 
Section 8.3.4 presents the implications related to the general high-frequency vocabulary given that it makes up a huge proportion of the TCM texts. Even though Section 8.3.1 has touched on the pedagogical suggestions for high-frequency vocabulary since a considerable number (1,757 word types) of technical words are from general highfrequency vocabulary, this subsection focuses on offering suggestions on developing learners' knowledge of general high-frequency vocabulary in its own right. This is based on the consideration that, in some EFL contexts, a majority of the TCM learners lack knowledge of general high-frequency vocabulary. The last subsection provides a summary of the pedagogical implications.

\subsubsection{Developing TCM learners' knowledge of technical words}

As indicated in the previous chapter, knowledge of technical vocabulary is critical to TCM learners in their specialized English development. The frequency-based TCM technical word list provides TCM learners and teachers a pool of lexical resources which might be particularly worth focusing on. However, it should be noted that working with the TCM technical word list is not simply about remembering those items by rote learning (Dang, 2017). Nor should the items in the lists be learned or taught in decontextualized ways as noted by previous research (Coxhead, 2000). Rather, these items should be learned in a principled manner according to their characteristics and learners' previous linguistic knowledge.

First, the TCM technical word list can be used as a guide for the teachers to establish longterm and short-term learning goals (Nation, 2013). At the beginning of the specialized course, learners and teachers can set the learning goals depending on learners' linguistic backgrounds or existing levels of general vocabulary as shown in Figure 8.1. As mentioned in Section 5.3.1, Level 1, 2 and 3 in Figure 8.1 represent TCM technical words from the first to the third 1,000 BNC/COCA levels, technical words outside the first to the third 1,000 BNC/COCA levels, and loan words, respectively. 
Figure 8.1: Learning sequence of the TCM technical word list

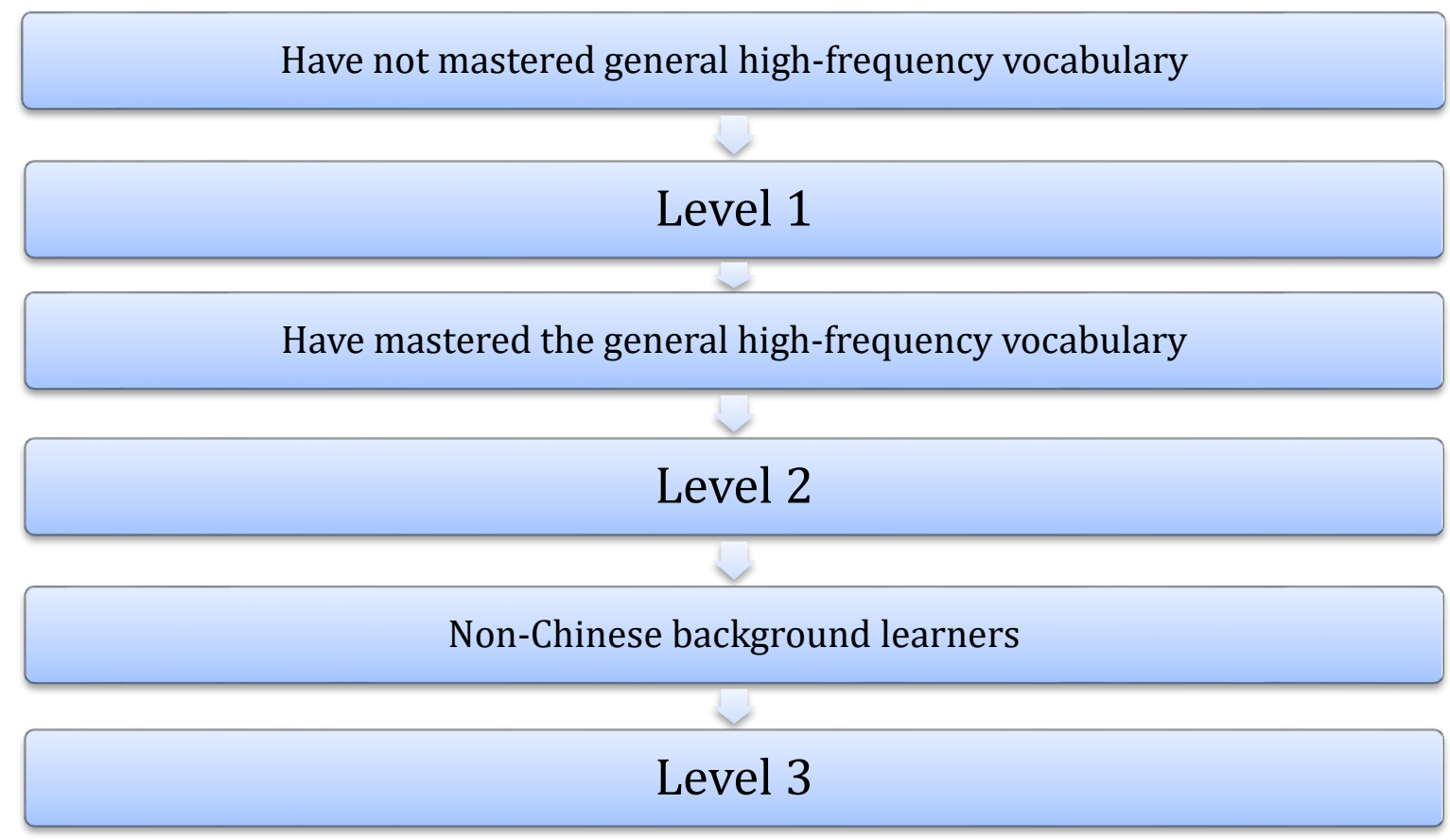

The different levels of each TCM technical sub-list allow the learners and teachers to focus on the technical vocabulary relevant to learners' existing linguistic knowledge. For example, learners who have mastered general high-frequency vocabulary can go straight to Level 2 technical vocabulary from each sub-list. Therefore, their goal of learning can start from Level 2 technical vocabulary. It is important to note that if the learners have mastered the general meanings of the general high-frequency vocabulary and not mastered the discipline-specific meanings of those words (see Section 8.3.2 for more details), they were considered as learners who have not mastered the general highfrequency vocabulary. In this case, their goal of technical vocabulary learning should include the learning of the discipline-specific meanings of the general high-frequency vocabulary, then proceed to Level 2, and Level 3 if the learners do not have knowledge of the loan words. In this way, an effective technical vocabulary learning program can be developed.

Second, once the learning goals have been established, the TCM technical word list can serve as a reference for a specialized English lexical syllabus in TCM. It is important for teachers to design materials and organize learning activities for learners to acquire/expand their knowledge of certain target words and consolidate such knowledge 
in meaningful ways. Nation's (2007) four strands, which involve meaning-focused input, language-focused learning, meaning-focused output, and fluency development, can act as a framework for designing a balanced specialized language course and organizing learning activities. To ensure effective learning, the teachers should first provide the learners with comprehensible meaning-focused input through listening and reading (Nation, 2013). Then, at the language-focused learning stage, the teachers need to draw learners' attention to items in the TCM technical word list explicitly. After that, the learners should be given opportunities to develop their knowledge through writing and speaking activities. The last strand is to develop learners' fluency over the items they have learned rather than to learn new items. Such fluency can be developed through all four skills - reading, listening, writing and speaking. Examples of activities for each strand can be found in Nation (2013) and Coxhead (2014).

Third, given that TCM is a lexically demanding discipline, helping learners to develop vocabulary learning strategies so that the learners are at a better position to learn the technical words autonomously is particularly valuable. Trainable vocabulary learning strategies include inferring from context, identifying lexical familiarization, unchaining nominal compounds, synonym search and word analysis (Williams, 1985). For example, the students can be asked to make direct discoveries as to how technical words are used in the specialized field of TCM with corpus-based materials (Hsu, 2013). In addition to vocabulary learning strategies, it is also crucial for the TCM learners to develop word study strategies such as knowledge of using word parts (Nation, 2013). This is because many technical medical words, as can be seen in this thesis, are made up of word roots and affixes. For example, cardiovascular is made up of the root cardi(o) (meaning heart), the affix vascul (meaning vessel) and the affix -ar (meaning pertaining to). The root cardi(o) can also be combined with other affix such as tocography to become cardiotocograph (Hutton, 2006). Thus, a knowledge of roots and affixes is of great value to medical students. It can help learners to learn unfamiliar words by relating these words to known roots and affixes, and also help them check whether an unfamiliar word has been successfully guessed from the contexts (Nation, 2013).

Further, lexical items in the TCM technical word list can also be used for guidance in the assessment and testing of technical vocabulary knowledge. For example, test designers 
can select items from the list to develop the lexical components of a test. It should be noted that testing and assessment of technical vocabulary knowledge should be aligned with the teaching and learning activities and the learning goals (Malmström, Pecorari, \& Shaw, 2018). For example, if the learning activities only involve the receptive knowledge of certain technical words, the assessment should be reflective of receptive dimensions of knowing the target words.

In sum, the TCM technical word list can be of great value for TCM learners, teachers, course designers, and material writers. Nonetheless, it is also important to note that the TCM technical word list is only a starting point for learning technical vocabulary. Further, students ultimately also need to develop knowledge of technical words with lower occurrences which were not included in the TCM technical word list after they master the items in the list using the strategies mentioned above. Another point to note is that the learning sequence provided in Figure 8.1 is based on the assumption that most learners have reasonable knowledge of general high-frequency vocabulary. It is also possible that in some EFL contexts a majority of the learners have a low vocabulary level of less than 2,000 word families (Dang, 2017; Henriksen \& Danelund, 2015; Webb \& Chang, 2012). In such cases, the learners should focus on developing knowledge of general high-frequency vocabulary as suggested in Section 8.3.4.

\subsubsection{Developing learners' knowledge of technical lexical bundles}

Since the TCM technical bundle list is a supplementary list to the TCM technical word list, it can act as a referential list in the learners' development of technical vocabulary. The learners can target the technical words as mentioned in Section 8.3.1, but they can look for the use of the target single words in the lexical bundle list to see which words co-occur frequently with the target words. For example, if the learners target qi, they can find the use of qi in the lexical bundle list such as lung qi, qi deficiency, and tonify qi. In this way, the learners can know about the contexts where the target words are used, and acquire a sound repertoire of recurrent word combinations in their discipline of study. Hence, they can be at a better position in achieving fluency in specialized language use. Moreover, the TCM lexical bundle list can also help raise awareness of the importance of lexical bundles in the specialized discourse among the teachers and material writers. Thus, they can include a focus on relevant bundles in the materials and classrooms. 
In brief, if the learners want to develop higher proficiency in their specialized discipline, the TCM technical bundles list can be a good resource. With these lexical bundles, the learners better understand how meaning and structure are built in their discipline than just single words.

\subsubsection{Integrating learners' sources of difficulty in word list implementation}

While the corpus-based word lists offer us important insights into what are useful to the learners based on which words they are expected to encounter and use frequently (Nation, 2016b), there is little in the way of guidance for teachers to help them choose items that should be explicitly taught in the classroom. By looking at learners' understanding of technical words drawn from the corpus-based TCM technical word list, this thesis provides some implications for selecting technical words to focus on.

First, this research reveals that the more specialized a technical word is, the more comprehension problems it may cause among the learners. This is because some technical words do not usually occur outside a field (e.g., qi), the learners have few chances to encounter them in their language learning experience. Therefore, it is an important indicator for prioritizing items from a word list for instruction. The teachers can prioritize the technical words that are novel to their learners' language learning experience for instruction. For most Western background learners, the loan words are highly specialized and are novel to them, thus these items might be good candidates for overt instruction in their classroom. Further, this research found that the learners found it difficult to differentiate loan words which have similar forms (zhong, zong and zheng). Teachers should thus be aware of possible confusions that can be created by such form similarity of loan words. As for Chinese L1 learners, the words which they seldom encounter in their previous English language learning experience such as technical words from lower frequency bands are especially challenging. Examples such as lumbar and borborygmus can be found in Section 6.3.1.1, which can hardly be found in non-medical texts. Thus, a specialized English teacher in this context can explicitly focus on such technical words. 
Second, this research indicates that the semantic feature of a technical word such as polysemy and metaphor is another criterion for selecting items from a word list for instruction. Given that the polysemous or metaphoric technical words are more likely to cause comprehension problems, the teachers should give some priority to such words in the classroom. For example, based on the finding that some Chinese background learners knew channel as TV channel but not channel in the body (see Section 6.3.1, p. 135), the teachers can focus on helping the learners to acquire the discipline-specific meaning of channel. Semantic elaboration tasks can be especially effective in the learning of new meaning on known words. For instance, the teachers can organize activities such as working out the similarity between the 'basic' word meaning and its extended meaning as a technical word. At the same time, the teachers can also teach the learners to use authentic language samples from the corpus such as the TCM Corpora used at the present research to acquire the discipline-specific meanings of the known word forms. Such kind of corpus-based teaching might help train autonomous students to take charge of their own learning processes (Hsu, 2013). Furthermore, given that a considerable number of general high-frequency words are metaphorical in different disciplinary contexts, raising students' awareness of metaphor can also be beneficial for specialized vocabulary learning (Boers, 2000; Juchem-Grundmann \& Krennmayr, 2010). It can provide the ESP learners with insight into the conceptual domain of their target subject.

In sum, this section offers recommendations for a teacher to decide which words to focus on in the classroom. In this way, the valuable classroom time spent on teaching technical vocabulary can yield the greatest benefit.

\subsubsection{Enhancing learners' knowledge of general high-frequency vocabulary}

In the Discussion chapter, the importance of general high-frequency vocabulary in specialized English learning was highlighted given its high coverage in TCM texts in general. This finding offers important pedagogical implications for the ESP learners. Many technical words, as illustrated in this thesis, were found in the lists of most frequent 3,000-word-family list of BNC/COCA and are in fact common in general English. Learners and teachers should be aware that having a large overall vocabulary and a good knowledge of general vocabulary can make it much easier for TCM learners to acquire the new discipline-specific meanings of those known word forms (Crossley, Salsbury, \& 
McNamara, 2010). In other words, good knowledge of the general high-frequency vocabulary lays a solid foundation for specialized vocabulary learning. Thus, if most learners in the specialized English course have not grasped high frequency vocabulary, teachers should help learners develop their general knowledge of the high-frequency vocabulary at the beginning of their study. However, if only a minority of learners have not mastered the general high-frequency vocabulary, the teachers can follow the suggestions made in Section 8.3.1 while the learners should make an effort to enhance or expand their knowledge of the high-frequency vocabulary.

In brief, this subsection emphasizes that helping the learners to gain or enhance knowledge of the general high-frequency vocabulary is essential to the TCM learners for the development of specialized vocabulary.

\subsubsection{Summary}

This section has presented the pedagogical implications of the present research. However, the pedagogical suggestions are largely for English L1 or Chinese L1 TCM learners from monolingual learning contexts. Thus, the generalisability of those pedagogical implications to multi-lingual learning contexts with students from mixed language backgrounds remains an open question. In the next section, the limitations of the present research will be presented.

\subsection{Limitations}

This section presents the limitations of the present research. It has four sub-sections, which discuss limitations related to corpora, loan words, Nation's (2012) BNC/COCA word lists, and practical issues respectively.

\subsubsection{Limitations related corpora}

The corpora used in the present research are not large by contemporary standards, which should be acknowledged as one weakness of this research. This is due to the lack of materials especially English-medium textbooks in TCM. For the same reason, the validation corpus is not as balanced as the corpora used to develop the TCM technical word list. The validation corpus contains only one textbook with the other texts being journal articles. However, this provides a possible direction for future research on discipline-specific word lists, which will be further discussion in Section 8.5. 
Second, it was felt that a sufficiently representative selection of texts was achieved. However, it is still possible that a different selection of textbooks and articles, or a broader (or indeed narrower) selection of textbooks and journal articles in TCM, would have yielded different results. Thus, I am aware that the corpora used in the current research may still not capture all the patterns of the language in the specialized discourse of TCM, nor represent them in precisely the correct proportions.

Third, the present research has limited the focus on two written genres of the specialized language - textbooks and journal articles - that the TCM students read in their study to address their primary lexical needs. This is based on the consideration that textbooks and journal articles are the most important materials that are required by TCM study. Those materials are good sources to explore the lexis in TCM. Due to time constraints, this thesis has not investigated the TCM lexis used in other types of texts and other registers. Thus, it should be regarded as a starting point in the exploration of TCM lexis, which provides a direction for future research as discussed in Section 8.5.

\subsubsection{Limitations caused by the Chinese loan words}

Given that TCM is a special discipline which originates from Chinese culture and philosophy, a large number of loan words (Romanized Chinese words) are used in the English-medium texts in this discipline. Fundamental differences between Chinese language and English language inevitably results in limitations. Since the pronunciation of many characters is identical, such characters appear in the same form. For example, Huang can be a common family name but can also be commonly seen as part of herbal medicine (e.g., Huang Bai, Huang Qi). Both uses of Huang occur frequently in the TCM Corpora. As Huang has been included in the proper noun list of Nation's (2012) BNC/COCA lists, it was thus kept in the proper noun list. However, this can have an influence on the coverage figures of relevant word lists such as the proper noun list.

The same problem also has an impact on the TCM technical word list. For example, the character shen (神) that means Spirit is different from shen (肾) which means kidney. In the actual texts, the relevant Chinese characters might be presented beside the word such as shen to make the distinction as illustrated above. Such Chinese characters, however, cannot be processed by the Range program and were thus removed. This means that they 
were counted as the same word 'shen' by the Range program and 'shen' as presented in the TCM technical word list can therefore refer to two concepts.

\subsubsection{Limitations related Nation's (2012) BNC/COCA word lists}

The third group of limitations is related to Nation's (2012) BNC/COCA word lists which were used in the lexical profile and vocabulary load analysis. First, when the present research was conducted, the $26^{\text {th }}$ to the $28^{\text {th }} 1,000$ word families of BNC/COCA word lists were still under development. Thus, only the first to the 25th BNC/COCA word lists and the four supplementary lists were used in the present research. The lexical profile analysis could have been more comprehensive if the $26^{\text {th }}$ to the $28^{\text {th }} 1,000$ word families had been incorporated in the analysis.

The second limitation related to Nation's (2012) BNC/COCA lists is the use of the abbreviation list. In this research, the abbreviation list (BASEWRD 34) of the BNC/COCA lists was used and adapted. However, it is not an ideal option to use an abbreviation list originally created from general English for specialized English. The results would have been more accurate if an abbreviation list for TCM was built from scratch at the beginning of the present research. One reason is that some of the abbreviations in the BNC/COCA abbreviation list should have been technical words in this specialized corpora. For example, $S H I$ is included in the abbreviation list (BASEWRD 34) which is actually a loan word borrowed from Chinese in the TCM Corpora. Misclassification occurred in this way. Even though SHI is moved from the abbreviation list to the loan word list, the work involved is not necessarily less than creating a new abbreviation list. Further, it is possible that some misclassifications were not discerned. A second reason is that the majority of abbreviations needs to be identified via concordance check and be manually added to the abbreviation list. This is because a large number of abbreviations used in the ESP texts are highly specialized. Therefore, it is very unlikely that they occur in the general English texts and thus in the abbreviation list of the BNC/COCA. For these reasons, instead of adopting the existing BNC/COCA abbreviation list, I would suggest that future ESP vocabulary research creates a new abbreviation list which does not necessarily involve more work but definitely leads to more accurate analysis. A possible way to handle the abbreviations in the specialized corpus is to identify technical abbreviations 
and create a technical abbreviation list as Coxhead and Demecheleer (2018) did for their Plumbing corpus.

\subsubsection{Limitations caused by practical issues}

In addition to limitations related to corpora, loan words and BNC/COCA lists, there is also a number of limitations caused by practical issues. First, the number of participants in the word association task and follow-up interview is relatively small due to the exploratory nature of that study. There are only 11 and 10 participants in the Chinese background group and Western background group, respectively. This is a fundamental shortcoming of the present research. The inferential results related to the word association task in Study 3 should thus be taken with caution. They should be regarded as tentative rather than conclusive.

Second, the word association task needs some improvement. For example, urine was used as an association option provided in item 13, and it was used as the cue word as item 14 . It was misleading to some learners when they saw the same item in a row that played different roles in the word association task. It would be better to reverse the sequence of item 13 and 14, and place them in different pages of the task. This problem was not identified until the data was collected.

Third, only one word association task was used in Study 3, which consisted of 20 items. This is a small number of items. It would be ideal to have more task items in the word association task or have more than one word association task so that richer data can be elicited in the follow-up interview and the related inferential data can be more powerful. Nevertheless, the present research has weighed up practical constraints of participants' time and capacity to complete longer tasks that could have been led to fatigue. Thus, it is noted as a limitation of the present research, which inevitably limits the generalizability of the findings related to the word association task.

\subsection{Directions for future research}

The findings and limitations of this research provide insights for future research. They can provide four different directions for TCM and vocabulary. The first direction of the present research is to look at the vocabulary used in other types of specialized language in TCM such as classroom discourse and multimodal texts. These texts are also important 
to the development of disciplinary literacy. The present research limited the focus on textbooks and journal articles, which has been acknowledged as a limitation above. However, vocabulary used in other kinds of texts (e.g., multimodal texts, clinical conversation, tutorials) also deserves attention in future research. Research that looks at the spoken discourse in the discipline of TCM can be especially valuable. In this way, the researchers, ESP teachers and learners can have a better understanding of the lexis in this specialized discipline. Similarly, it is also important to look at the productive dimension of technical vocabulary in TCM and other disciplines. For example, what technical vocabulary do TCM learners use in their assignments or other tasks that they produce?

The second direction is to further validate the TCM technical word list and the TCM technical lexical bundle list. As mentioned above, due to a lack of English-medium materials in this discipline, the validation corpus is not well balanced. It would be desirable for future research on discipline-specific word lists to develop a validation corpus which mirrors the structure of the specialized corpora from which the disciplinespecific word list is developed. In addition, other validating sources such as teachers' perception of the word usefulness and learners' vocabulary knowledge can also provide insights into the validity of the word lists (Dang, 2017).

The third direction is to explore the difficulty that non-Chinese EFL learners have in the learning of technical vocabulary in their study. The present research has investigated the difficulty in the understanding of TCM technical words from the perspectives of Chinese L1 learners and English L1 learners. During the time of data collection in China, I found that a huge number of international students who are studying TCM in Chinese TCM universities but using English as the medium of study. Those learners are neither English L1 speakers nor Chinese L1 speakers. Due to the learners' time constraints, they did not participate in the current study. However, it would be interesting to investigate in future research what difficulties they have in their study and how they understand the technical words they encounter frequently.

Lastly, more empirical information about how the ability to comprehend and use technical vocabulary develops in different students across different stages of study and 
how such development can be fostered is still needed. It would be interesting in further research to test various participants' comprehension of the TCM texts plus their uptake of technical vocabulary from the texts since it is well known that incidental vocabulary acquisition from textual input correlates positively with text comprehension. It is even more interesting to explore whether the Chinese background readers (EFL learners) of TCM texts struggle more with text comprehension, despite their initial greater familiarity with culture-specific notions therein. In addition, what kinds of technical vocabulary pose the most difficulty to the learners in other academic disciplines such as medicine and engineering also remains very much open and an important priority for future work.

\subsection{Conclusion}

In sum, this thesis has bridged a gap in the ESP literature through a thorough investigation of vocabulary in TCM, which is an under-researched discipline. The development of TCM technical word lists in the form of both single and multiword units has shed light on the nature of technical vocabulary and provided an important basis for future research into vocabulary in ESP. In addition, this thesis has provided valuable pedagogical insights into the use of inevitably long word lists in the limited classroom time depending on learners' linguistic backgrounds and proficiency. Overall, these investigations lay a foundation for further research into specialized language use in TCM, technical vocabulary in other disciplines, as well as ESP education especially that in China. 


\section{References}

Ackermann, K., \& Chen, Y. H. (2013). Developing the Academic Collocation List (ACL) - A corpus-driven and expert-judged approach. Journal of English for Academic Purposes, 12(4), 235-247. doi:10.1016/j.jeap.2013.08.002

Ädel, A., \& Erman, B. (2012). Recurrent word combinations in academic writing by native and non-native speakers of English: A lexical bundles approach. English for Specific Purposes, 31(2), 81-92. doi:10.1016/j.esp.2011.08.004

Ardasheva, Y., \& Tretter, T. R. (2015). Developing science-specific, technical vocabulary of high school newcomer English learners. International Journal of Bilingual Education and Bilingualism, 1-20.

Arnon, I., \& Snider, N. (2010). More than words: Frequency effects for multi-word phrases. Journal of Memory and Language, 62, 67-82.

Barcroft, J. (2004). Effects of sentence writing in second language lexical acquisition. Second Language Research, 20(4), 303-334.

Barcroft, J. (2015). Lexical input processing and vocabulary learning. Amsterdam: John Benjamins.

Basturkmen, H. (2006). Ideas and options in English for specific purposes. Mahwah, New Jersey: Lawrence Erlbaum Assoicates Publishers.

Bauer, L., \& Nation, P. (1993). Word families. International Journal of Lexicography, 6(4), 253-279.

Berman, R., \& Cheng, L. (2010). English academic language skills: Perceived difficulties by undergraduate and graduate students, and their academic achievement. Canadian Journal of Applied Linguistics, 4(1-2), 25-40.

Bertels, A., \& Speelman, D. (2014). Clustering for semantic purposes: Exploration of semantic similarity in a technical corpus. Terminology, 20(2), 279-303. doi:10.1075/term.20.2.07ber

Biber, D. (1993). Representativeness in corpus design. Literary and Linguistic Computing, $8(4), 243-257$.

Biber, D. (2006). University language: A corpus-based study of spoken and written registers. Amsterdam: John Benjamins. 
Biber, D. (2009). A corpus-driven approach to formulaic language in English: Multi-word patterns in speech and writing. International Journal of Corpus Linguistics, 14(3), 275-311. doi:10.1075/ijcl.14.3.08bib

Biber, D., \& Barbieri, F. (2007). Lexical bundles in university spoken and written registers. English for Specific Purposes, 26, 263-286.

Biber, D., Conrad, S., \& Cortes, V. (2004). If you look at... : Lexical bundles in university teaching and textbooks. Applied Linguistics, 25(3), 371-405. doi:DOI 10.1093/applin/25.3.371

Biber, D., Conrad, S., \& Reppen, R. (1994). Corpus-based approaches to issues in applied linguistics. Applied Linguistics, 15, 169-189.

Biber, D., Johansson, S., Leech, G., Conrad, S., \& Finegan, E. (1999). The Longman Grammar of Spoken and Written English. London: Longman.

Boers, F. (1999). When a bodily source domain becomes prominent: The joy of counting metaphors in the socio-economic domain. In R. W. Gibbs \& G. J. Steen (Eds.), Metaphor in Cognitive Linguistics (pp. 47-56). Amsterdam: John Benjamins.

Boers, F. (2000). Enhancing metaphoric awareness in specialised reading. English for Specific Purposes, 19, 137-147.

Boers, F., \& Demecheleer, M. (1997). A few metaphorical models in (Western) economic discourse. In W. A. Liebert, G. Redeker, \& L. Waugh (Eds.), Discourse and Perspective in Cognitive Linguistics (pp. 115-129). Amsterdam: John Benjamins.

Borrell-Carrio, F., Suchman, A. L., \& Epstein, R. M. (2004). The biopsychosocial model 25 years later: Principles, practice, and scientific inquiry. Annals of Family Medicine, 2(6), 576-582. doi:10.1370/afm.245

Brezina, V., \& Gablasova, D. (2015). Is There a Core General Vocabulary? Introducing the New General Service List. Applied Linguistics, 36(1), 1-22. doi:10.1093/applin/amt018

Browne, C. (2014). A New General Service List: The better mousetrap we've been looking for? Vocabulary learning and Instruction, 3(1), 1-10. doi:10.7820/vli.v03.1.browne

Browne, C., Culligan, B., \& Phillips, J. (n.d.). A new academic word list. Retrieved from http://www.newacademicwordlist.org/

Byrd, P., \& Coxhead, A. (2010). On the other hand: Lexical bundles in academic writing and in the teaching of EAP. University of Sydney Papers in TESOL, 5, 31-64.

Cameron, L. (2003). Metaphor in educational discourse. London: MPG Books Ltd. 
Campion, M. E., \& Elley, W. B. (1971). An academic vocabulary list. Wellington: New Zealand Council for Educational Research.

Carter, R., \& McCarthy, M. (2006). Cambridge grammar of English. Cambridge: Cambridge University Press.

Charteris-Black, J. (2000). Metaphor and vocabulary teaching in ESP economics. English for Specific Purposes, 19, 149-165.

Charteris-Black, J., \& Musolff, A. (2003). 'Battered hero' or 'innocent victim'? A comparative study of metaphors for euro trading in British and German financial reporting. English for Specific Purposes, 22, 153-176.

Chen, Q., \& Ge, G.-c. (2007). A corpus-based lexical study on frequency and distribution of Coxhead's AWL word families in medical research articles (RAs). English for Specific Purposes, 26(4), 502-514. doi:10.1016/j.esp.2007.04.003

Chung, T. M. (2003). A corpus comparison approach for terminology extraction. Terminology, 9(2), 221-246.

Chung, T. M., \& Nation, P. (2003). Technical vocabulary in specialised texts. Reading in a foreign language, 15(2), 103-116.

Chung, T. M., \& Nation, P. (2004). Identifying technical vocabulary. System, 32(2), 251263.

Cohen, A. D., Glasman, B., Rosenbaum-Cohen, P. R., Ferrara, J., \& Fine, J. (1979). Reading English for specialized purposes: Discourse analysis and the use of student informants. TESOL Quarterly, 13, 551-564.

Cohen, L., Manion, L., \& Morrison, K. (2007). Research methods in education (6th ed.). Abingdon: Routledge.

Cortes, V. (2004). Lexical bundles in published and student disciplinary writing: Examples from history and biology. English for Specific Purposes, 23(4), 397-423. doi:10.1016/j.esp.2003.12.001

Coxhead, A. (2000). A new academic word list. TESOL Quarterly, 34(2), 213-238. doi:Doi $10.2307 / 3587951$

Coxhead, A. (2011). The Academic Word List 10 Years On: Research and Teaching Implications. TESOL Quarterly, 45(2), 355-362. doi:10.5054/tq.2011.254528

Coxhead, A. (2014). New ways in teaching vocabulary. Alexandria: TESOL Inc.

Coxhead, A. (2018). Vocabulary and English for Specific Purposes Research. London: Routledge. 
Coxhead, A., Dang, T. N. Y., \& Mukai, S. (2017). Single and multi-word unit vocabulary in university tutorials and laboratories: Evidence from corpora and textbooks. Journal of English for Academic Purposes, 30, 66-78. doi:10.1016/j.jeap.2017.11.001

Coxhead, A., \& Demecheleer, M. (2018). Investigating the technical vocabulary of Plumbing. English for Specific Purposes, 51, 84-97. doi:10.1016/j.esp.2018.03.006

Coxhead, A., Demecheleer, M., \& McLaughlin, E. (2016). The technical vocabualry of Carpentry: Loads, lists and bearings. TESOLANZ Journal, 24, 38-71.

Coxhead, A., \& Hirsh, D. (2007). A pilot science-specic word list. Revue Francaise de Linguistique Appliqueé, 7(2), 65-78.

Coxhead, A., Stevens, L., \& Tinkle, J. (2010). Why might secondary science textbooks be difficult to read? New Zealand Studies in Applied Linguistics, 16(2), 37-52.

Crossley, S., Salsbury, T., \& McNamara, D. (2010). The Development of Polysemy and Frequency Use in English Second Language Speakers. Language Learning, 60(3), 573-605. doi:10.1111/j.1467-9922.2010.00568.x

Csomay, E., \& Petrović, M. (2012). "Yes, your honor!”: A corpus-based study of technical vocabulary in discipline-related movies and TV shows. System, 40(2), 305-315. doi:10.1016/j.system.2012.05.004

Dahm, M. R. (2011). Exploring perception and use of everyday language and medical terminology among international medical graduates in a medical ESP course in Australia. English for Specific Purposes, 30, 186-197. doi:http://dx.doi.org/10.1016/i.esp.2011.02.004

Dang, T. N. Y. (2017). Investigating vocabulary in academic spoken English: Corpus, teachers, and learners (Unpublished doctoral thesis). Victoria University of Wellington, Wellington.

Dang, T. N. Y., Coxhead, A., \& Webb, S. (2017). The Academic Spoken Word List. Language Learning, 67(4), 959-997. doi:10.1111/lang.12253

Dang, T. N. Y., \& Webb, S. (2014). The lexical profile of academic spoken English. English for Specific Purposes, 33, 66-76. doi:10.1016/j.esp.2013.08.001

Dang, T. N. Y., \& Webb, S. (2016). Making an essential word list for beginners. In P. Nation (Ed.), Making and Using Word Lists for Language Learning and Testing (pp. 153167). Amsterdam: John Benjamins. 
Davies, M. (2008-). The Corpus of Contemporary American English (COCA): 560 million words, 1990-present. from Available online at https://corpus.byu.edu/coca/

Douglas, D. (2000). Assessing Language for Specific Purposes. Cambridge: Cambridge University Press.

Dudley-Evans, T., \& St John, M. (1998). Developments in English for Specific Purposes. Cambridge: Cambridge University Press.

Durrant, P. (2009). Investigating the viability of a collocation list for students of English for academic purposes. English for Specific Purposes, 28(3), 157-169. doi:10.1016/j.esp.2009.02.002

Durrant, P. (2013). Discipline and Level Specificity in University Students' Written Vocabulary. Applied Linguistics, 35(3), 328-356. doi:10.1093/applin/amt016

Durrant, P. (2016). To what extent is the Academic Vocabulary List relevant to university student writing? English for Specific Purposes, 43, 49-61.

Durrant, P., \& Mathews-Aydınlı, J. (2011). A function-first approach to identifying formulaic language in academic writing. English for Specific Purposes, 30(1), 58-72.

Ellis, N. C., Simpson-Vlach, R., \& Maynard, C. (2008). Formulaic language in native and second-language speakers: Psycholinguistics, corpus linguistics, and TESOL. TESOL Quarterly, 41, 375-396.

Evans, S., \& Green, C. (2007). Why EAP is necessary: A survey of Hong Kong tertiary students. Journal of English for Academic Purposes, 6(1), 3-17.

Evans, S., \& Morrison, B. (2011). Meeting the challenges of English-medium higher education: The first-year experience in Hong Kong. English for Specific Purposes, 30(3), 198-208. doi:10.1016/j.esp.2011.01.001

Farrell, P. (1990). Vocabulary in ESP: A lexical Analysis of the English of Electronics and a Study of Semi-Technical Vocabulary. CLCS Occassional Paper No.25: Trinity College.

Fauci, A. S., Braunwald, E., Kasper, D. L., Hauser, S. L., Longo, D. L., Jameson, J. L., \& Loscalzo, J. (2008). Harrison's principles of internal medicine (17th ed.). New York: McGrawHill.

Ferguson, G. (2012). English for medical purposes. In B. Paltridge \& S. Starfield (Eds.), The handbook of English for specific purposes (pp. 243-261). Malden MA: John Wiley \& Sons.

Fitzpatrick, T. (2006). Habits and rabbits: Word associations and the L2 lexicon. EUROSLA Yearbook, 6, 121-145. 
Fitzpatrick, T., \& Izura, C. (2011). Word association in L1 and L2: An exploratory study of response types, response times and inter-language mediation. Studies in Second Language Acquisition, 33(03), 373-398.

Francis, W. N., \& Kučera, H. (1982). Frequency analysis of English usage: Lexicon and grammar. Boston: Houghton Mifflin.

Fraser, S. (2005). The lexical characteristics of specialized texts. In K. Bradford-Watts, C. Ikeguchi, \& M. Swanson (Eds.), JALT2004 conference proceedings. Tokyo: JALT.

Fraser, S. (2007). Providing ESP learners with the vocabulary they need: Corpora and the creation of specialized word lists. Hiroshima Studies in Language and Language Education(10), 127-143.

Fraser, S. (2009). Breaking down the divisions between general, academic and technical vocabulary: The establishment of a single, discipline-based word list for ESP learners. Hiroshima Studies in Language and Language Education, 12, 151-167.

Fraser, S. (2012). Factors affecting the learning of technical vocabulary. Hiroshima Studies in Language and Language Education, 15, 123-142.

Fraser, S. (2013). Building corpora and compiling pedagogical lists for university medical students. Hiroshima Studies in Language and Language Education(16), 65-88.

Gablasova, D. (2015). Learning technical words through L1 and L2: Completeness and accuracy of word meanings. English for Specific Purposes, 39, 62-74.

Gardner, D. (2013). Exploring vocabulary: Language in action. New York: Routledge.

Gardner, D., \& Davies, M. (2014). A new academic vocabulary list. Applied Linguistics, 35(3), 305-327. doi:http://dx.doi.org/10.1093/applin/amt015.

Ghadessy, P. (1979). Frequency counts, words lists, and materials preparation: A new approach. English Teaching Forum, 17(1), 24-27.

Gilner, L. (2011). A primer on the General Service List. Reading in a foreign language, 23(1), 65-83.

Goldman, L., \& Ausiello, D. (2008). Cecil textbook of internal medicine (23rd ed.). Philadelphia: Saunders Elsevier.

Ha, A. Y. H., \& Hyland, K. (2017). What is technicality? A Technicality Analysis Model for EAP vocabulary. Journal of English for Academic Purposes, 28, 35-49. doi:10.1016/j.jeap.2017.06.003 
Ha, Y.-H. (2015). Technical vocabulary in finance: A corpus-based study of annual reports and earnings calls. A thesis submitted in partial fulfilment of the requirements for the degree of Doctor of Philosophy at The University of Hong Kong.

heart. (n.d.). In Merriam-Webster.com. Retrieved from August 27, 2017 https://www.merriam-webster.com/dictionary/heart

Heatley, A., Nation, P., \& Coxhead, A. (2002). Range: A program for the analysis of vocabulary in texts.

Henriksen, B., \& Danelund, L. (2015). Studies of Danish L2 learners' vocabulary knowledge and the lexical richness of their written production in English. In P. Pietilä, K. Doró, \& R. Pípalová (Eds.), Lexical issues in L2 writing (pp. 29-56). Newcastle upon Tyne: Cambridge Scholars Publishing.

Hirvela, A. (1997). Disciplinary portfolios and EAP writing Instruction. English for Specific Purposes, 16(2), 83-100.

Hoffmann, L. (1981). The linguistic analysis and teaching of LSP in the German Democratic Republic. In J. Hoedt \& R. Turner (Eds.), The world of LSP, and new bearings in LSPV (pp. 25-47). Copenhagen: UNESCO/ALSED LSP Network and Newsletter, the Copenhagen School of Economics.

Holliday, A. (2010). Analysing qualitative data. In B. Paltridge \& A. Phakiti (Eds.), Continuum Companion to Research Methods in Applied Linguistics. London/New York: Continuum International Publishing Group.

Hsu, W. H. (2011). The vocabulary thresholds of business textbooks and business research articles for EFL learners. English for Specific Purposes, 30(4), 247-257. doi:10.1016/j.esp.2011.04.005

Hsu, W. H. (2013). Bridging the vocabulary gap for EFL medical undergraduates: The establishment of a medical word list. Language Teaching Research, 17(4), 454-484. doi:10.1177/1362168813494121

Hsu, W. H. (2014a). Measuring the vocabulary load of engineering textbooks for EFL undergraduates. English for Specific Purposes, 33, 54-65. doi:10.1016/j.esp.2013.07.001

Hsu, W. H. (2014b). The most frequent opaque formulaic sequences in English-medium college textbooks. System, 47, 146-161. doi:10.1016/j.system.2014.10.001 
Hsu, W. H. (2016). Measuring the Vocabulary Load of English-medium Traditional Chinese Medicine (TCM) Textbooks. Paper presented at the 3rd International Conference on Social Science, Shanghai.

Hu, M., \& Nation, I. S. P. (2000). Vocabulary density and reading comprehension. Reading in a foreign language, 13(1), 403-430.

Hu, Y.-H., \& Fong, Y.-Y. (2010). Obstacles to CM-guided L2 idiom interpretation. In S. D. Knop, F. Boers, \& A. D. Rycker (Eds.), Fostering language teaching efficiency through cognitive linguistics. Berlin and New York: Hubert \& Co. GmbH \& Co. KG, Gottingen.

Hutchinson, T., \& Waters, A. (1985). ESP at the crossroads. In J. Swales (Ed.), Episodes in ESP (pp. 177-187). Oxford: Pergamon.

Hutton, A. R. (2006). An introduction to medical terminology for health care: A selfteaching package. London: Churchill Livingstone.

Hyland, K. (2008). As can be seen: Lexical bundles and disciplinary variation. English for Specific Purposes, 27(1), 4-21. doi:10.1016/j.esp.2007.06.001

Hyland, K. (2016). General and specific EAP. In K. Hyland \& P. Shaw (Eds.), The Routledge handbook of English for academic purposes. London: Routledge.

Hyland, K., \& Tse, P. (2007). Is there an academic word list. TESOL Quarterly, 41(No. 2), 235-253.

Juchem-Grundmann, C., \& Krennmayr, T. (2010). Corpus-informed integration of metaphor in materials for the business English clasroom. In S. D. Knop, F. Boers, \& A. D. Rycker (Eds.), Fostering language teaching efficiency through cognitive linguistics (pp. 317-335). Berlin and New York: Hubert \& Co. GmbH \& Co. KG, Gottingen.

Juilland, A. G., Brodin, D. R., \& Davidovitch, C. (1970). Frequency dictionary of French words. The Hague: Mouton de Gruyter.

Kageura, K., \& Umino, B. (1996). Methods of automatic term recognition: A review. Terminology, 3, 259-289.

Kaptchuk, T. (2001). Chinese Medicine: The Web That has no weaver. London: Rider.

Kennedy, G. (2003). Structure and meaning in English: A guide for teachers. U.K.: Pearson Education Limited. 
Khani, R., \& Tazik, K. (2013). Towards the Development of an Academic Word List for Applied Linguistics Research Articles. RELC Journal, 44(2), 209-232. doi:10.1177/0033688213488432

Konstantakis, N. (2007). Creating a business word list for teaching business English. Elia, 7, 79-102.

Kremmel, B. (2016). Word Families and Frequency Bands in Vocabulary Tests: Challenging Conventions. TESOL Quarterly, 50(4), 976-987. doi:10.1002/tesq.329

Kwary, D. A. (2011). A hybrid method for determining technical vocabulary. System, 39(2), 175-185.

Lakoff, G., \& Johnson, M. (1980). Metaphors We Live By. Chicago: University of Chicago Press.

Laufer, B. (1997). What's in a word that make it hard or easy: some intralexical factors that affect the learning of words. In N. Schmitt \& M. McCarthy (Eds.), Vocabulary: Description, Acquisition, and Pedagogy (pp. 140-155). Cambridge: Cambridge University Press.

Laufer, B. (1998). The development of passive and active vocabulary in a second language: Same or different? Applied Linguistics, 19(2), 255-271.

Laufer, B., \& Ravenhorst-Kalovski, G. C. (2010). Lexical threshold revisited: Lexical text coverage, learners' vocabulary size and reading comprehension. Reading in a Foreign. Language, 22(1), 15-30.

Laufer, B., \& Sim, D. (1985). Measuring and explaining the reading threshold needed for English for academic purposes texts. Foreign Language Annals, 18(5), 405-411.

Lei, L., \& Liu, D. L. (2016). A new medical academic word list: A corpus-based study with enhanced methodology. Journal of English for Academic Purposes, 22, 42-53. doi:10.1016/j.jeap.2016.01.008

Li, Y., \& Qian, D. D. (2010). Profiling the Academic Word List (AWL) in a financial corpus. System, 38(3), 402-411. doi:10.1016/j.system.2010.06.015

Lindstromberg, S. (1991). Metaphor and ESP: A ghost in the machine? Journal of English for Specific Purposes, 10, 207-225.

Littlemore, J. (2009). Applying cognitive linguistics to second language learning and teaching. Basingstoke, UK: Palgrave Macmillan.

Littlemore, J., Chen, P., Tang, P. L., Koester, A., \& Barnden, J. (2010). The use of metaphor and metonymy in academic and professional discourse and theri challenges for 
learners and teachers of English. In S. D. Knop, F. Boers, \& A. D. Rycker (Eds.), Fostering language teaching efficiency through cognitive linguistics (pp. 333-349). Berlin and New York: Hubert \& Co. GmbH \& Co. KG, Gottingen.

Littlemore, J., \& Low, G. (2006). Metaphoric competence, second language learning, and communicative language ability. Applied Linguistics, 27(2), 268-294.

Liu, D. L. (2012). The most frequently-used multi-word constructions in academic written English: A multi-corpus study. English for Specific Purposes, 31(1), 25-35. doi:10.1016/j.esp.2011.07.002

Liu, J., \& Han, L. N. (2015). A corpus-based environmental academic word list building and its validity test. English for Specific Purposes, 39, 1-11. doi:10.1016/j.esp.2015.03.001

Low, G., Littlemore, J., \& Koester, A. (2007). Metaphor use in three UK university lectures. Applied Linguistics, 29(3), 428-455. doi:10.1093/applin/amn008

Lu, C. (2014). English for Chinese Medicine Purpose: A preliminary needs analysis from the perspective of former students and course instructors. (Unpublished Master's dissertation), The University of Exeter, Exeter, U.K.

Lu, C., \& Durrant, P. (2017). A corpus-based lexical analysis of Chinese medicine research articles. The Asian Journal of Applied Linguistics, 4(1), 3-15.

Lynn, R. W. (1973). Preparing word-lists: A suggested method. RELC Journal, 4(1), 25-28. Maciocia, G. (2005). The Foundations of Chinese Medicine: A Comprehensive Text for Acupuncturists and Herbalists (Second ed.). London: Elsevier Churchill Livingston.

Malmström, H., Pecorari, D., \& Shaw, P. (2018). Words for what? Contrasting university students' receptive and productive academic vocabulary needs. English for Specific Purposes, 50, 28-39.

Martínez, I. A., Beck, S. C., \& Panza, C. B. (2009). Academic vocabulary in agriculture research articles: A corpus-based study. English for Specific Purposes, 28(3), 183198.

Martínez, R. (2013). A framework for the inclusion of multi-word expressions in ELT. ELT Journal, 67(2), 184-198.

Martinez, R., \& Murphy, V. A. (2011). Effect of frequency and idiomaticity on second language reading comprehension. TESOL Quarterly, 45(2), 267-290.

Martinez, R., \& Schmitt, N. (2012). A phrasal expressions list. Applied Linguistics, 33(3), 299-320. doi:10.1093/applin/ams010 
Meara, P. (2009). Connected Words: word associations and second language lexical acquisition. Amsterdam: John Benjamins.

Mežek, S. p. (2013). Learning terminology from reading texts in English: The effects of note-taking strategies. Nordic Journal of English Studies, 13(1), 133-161.

Miller, D., \& Biber, D. (2015). Evaluating reliability in quantitative vocabulary studies: The influence of corpus design and composition. International Journal of Corpus Linguistics, 20(1), 30-53.

Monroe, E. E., \& Panchyshyn, R. (1995). Vocabulary considerations for teaching mathematics. Childhood Education, 72(2), 80-83.

Morris, E., Bransford, J. D., \& Franks, J. J. (1977). Levels of processiong versus transfer appropriate processing. Journal of Verbal Learning and Verbal behavior, 16, 519533.

Mudraya, O. (2006). Engineering English: A lexical frequency instructional model. English for Specific Purposes, 25(2), 235-256. doi:10.1016/j.esp.2005.05.002

Mungra, P., \& Canziani, T. (2013). Lexicographic studies in medicine: Academic Word List for clinical case histories. Ibérica, 25, 39-62.

Nation, P. (2001). Learning vocabulary in another language. Cambridge: Cambridge Universith Press.

Nation, P. (2004). A study of the most frequent word families in the British National Corpus. In P. Bogaards \& B. Laufer (Eds.), Vocabulary in a second language: Selection, acquisition, and testing Amsterdam: John Benjamins.

Nation, P. (2006). How large a vocabulary is needed for reading and listening? Canadian Modern Language Review, 63(1), 59-82.

Nation, P. (2007). The four strands. Innovation in Language Learning and Teaching, 1(1), $1-12$.

Nation, P. (2012). The BNC/COCA word family lists. Retrieved from http://www.victoria.ac.nz/lals/about/staff/paul-nation.

Nation, P. (2013). Learning vocabulary in another Language (2nd ed.). Cambridge: Cambridge University Press.

Nation, P. (2016a). Hyphenated words and transparent compounds. In P. Nation (Ed.), Making and using word lists for language learning and testing. Amsterdam/Philadephia: John Benjamins Publishing Company. 
Nation, P. (2016b). Making and using word lists for language learning and testing. Amsterdam/Philadephia: John Benjamins Publishing Company.

Nation, P., Coxhead, A., Chung, T. M., \& Quero, B. (2016). Specialized word lists. In P. Nation (Ed.), Making and Using Word Lists for Language Learning and Testing. Amsterdam: John Benjamins.

Nation, P., \& Hwang, K. (1995). Where would general service vocabulary stop and special purpose vocabulary begin? System, 23, 35-41.

Nation, P., \& Waring, R. (1997). Vocabulary size, text coverage and word lists. In N. Schmitt \& M. McCarthy (Eds.), Vocabulary: Description, Acquisition, and Pedagogy. Cambridge: Cambridge University Press.

Nattinger, J. R., \& DeCarrico, J. S. (1992). Lexical phrases and language teaching. Oxford: Oxford University Press.

Nazar, R. (2016). Distributional analysis applied to terminology extraction: First results in the domain of psychiatry in Spanish. Terminology, 22, 141-170.

Nguyen, T. M. H., \& Webb, S. (2017). Examining second language receptive knowledge of collocation and factors that affect learning. Language Teaching Research, 21(3), 298-320.

Nurweni, A., \& Read, J. (1999). The English vocabulary knowledge of Indonesian university students. English for Specific Purposes, 18(2), 161-175.

Partington, A. (1998). Patterns and meaning. Using corpora for English language research and teaching. Amsterdam: John Benjamins Publishing Company.

Periñán-Pascual, C. (2015). The underpinnings of a composite measure for automatic term extraction: The case of SRC. Terminology, 21(2), 151-179. doi:10.1075/term.21.2.02per

Peruzzo, K. (2014). Term extraction and management based on event templates: An empirical study on an EU corpus. Terminology, 20(151-170).

Peters, P., \& Fernández, T. (2013). The lexical needs of ESP students in a professional field. English for Specific Purposes, 32(4), 236-247. doi:10.1016/j.esp.2013.05.002

Pinchbeck, G. G. (2014). Lexical frequency profiling of a large sample of Canadian high school diploma exam expository writing: L1 and L2 academic English. Paper presented at the American Association of Applied Linguistics, Portland, OR, USA.

Plag, I. (2003). Word-formation in English. Cambridge: Cambridge University Press. 
Porter, R. S., \& Kaplan, J. L. (2011). The Merck manual of diagnosis and therapy (19th ed.). NJ: Merck Sharp \& Dohme Corp.

Praninskas, J. (1972). American university word list. London: Longman.

protein. (n.d.). In Merriam-Webster.com. Retrieved from April 19, 2018, https://www.merriam-webster.com/dictionary/stroke

Pueyo, I. G., \& Val, S. (1996). The construction of technicality in the field of plastics: A functional approach towards teaching technical terminology. English for Specific Purposes, 15(4), 251-278.

Qian, D. D. (1999). Assessing the roles of depth and breadth of vocabulary knowledge in reading comprehension. Canadian Modern Language Review, 56, 282-308.

Qian, D. D., \& Schedl, M. (2004). Evaluating an in-depth vocabulary knowledge measure for assessing reading performance. Language Testing, 21, 28-52.

Quero, B. (2015). Estimating the vocabulary size of L1 Spanish ESP learners and the vocabulary load of medical textbooks. A thesis submitted to Victoria University of Wellington in fulfilment of the requirements for the degree of Doctor of Philosophy in Applied Linguistics.

Quero, B., \& Coxhead, A. (2018). Using a corpus-based approach to select medical vocabulary for an ESP course: The case for high-frequency vocabulary. In Y. Kirkgöz \& K. Dikilitaş (Eds.), Key issues in English for Specific Purposes in higher education (pp. 51-75). New York: Springer.

Read, J. (1993). The Development of a new measure of L2 vocabulary knowledge. Language Testing, 10, 355-371.

Read, J. (1998). Validating a test to measure depth of vocabulary knowledge. In A. Kunnan (Ed.), Validation in Languge Assessment (pp. 41-60). Mahwah, NJ: Lawrence Erlbaum.

Read, J. (2012). Piloting vocabulary tests. In G. Fulcher \& F. Davidson (Eds.), The Routledge Handbook of Language Testing (pp. 307-320). London: Routledge.

Richards, J. C. (1974). Word lists: Problems and prospects. RELC Journal, 5(2), 69-84.

Richards, J. C. (1976). The role of vocabulary teaching. TESOL Quarterly, 10(1), 77-89.

Salager, F. (1983). The lexis of fundamental medical English: Classificatory framework and rhetorical functions (A statistical approach). Reading in a foreign language, 1(1), 54-64. 
Salager-Meyer, F. (1990). Metaphors in medical English prose: A comparative study with French and Spanish. English for Specific Purposes, 9, 145-159.

Schmitt, N. (2000). Vocabulary in language learning. Cambridge: Cambridge University Press.

Schmitt, N. (2010a). Key issues in teaching and learning vocabulary. In R. Chacón-Beltrán, C. Abello-Contesse, \& M. d. M. Torreblanca-López (Eds.), Insights into non-native vocabulary teaching and learning (pp. 28-40). U.K.: Multilingual Matters.

Schmitt, N. (2010b). Researching vocabulary: A vocabulary research manual. New York: Palgrave Macmillan.

Schmitt, N., Jiang, X. Y., \& Grabe, W. (2011). The Percentage of Words Known in a Text and Reading Comprehension. Modern Language Journal, 95(1), 26-43. doi:10.1111/j.1540-4781.2011.01146.x

Schmitt, N., Ng, J. W. C., \& Garras, J. (2011). The word associates format: Validation evidence. Language Testing, 28(1), 105-126. doi:10.1177/0265532210373605

Schmitt, N., \& Schmitt, D. (2014). A reassessment of frequency and vocabulary size in L2 vocabulary teaching. Language Teaching, 47(04), 484-503.

Schmitt, N., \& Zimmerman, C. B. (2002). Derivative word forms: What do learners know? TESOL Quarterly, 36(2), 145-171.

Scott, M. (1997). PC analysis of key words - And key key words. System, 25(2), 233-245. doi:http://dx.doi.org/10.1016/S0346-251X(97)00011-0

Scott, M. (2006). The importance of key words for LSP. In E. Arno, A. Cervera, \& C. Ramos (Eds.), Information technologh in languages for specific purposes: Issues and prospects (pp. 231-243). Berlin: Springer.

Scott, M. (2009). In search of a bad reference corpus. In D. Archer (Ed.), What's in a wordlist? Investigating word frequency and keyword extraction (pp. 79-91). Oxford: Ashgate.

Scott, M. (2010). Problems in investigating keyness, or clearing the undergrowth and marking out trails... In M. Bondi \& M. Scott (Eds.), Keyness in texts (pp. 43-58). Amsterdam/Philadelphia: John Benjamins Publishing Company.

Scott, M. (2012). WordSmith tools version 6. Liverpool: Lexical Analysis Software Ltd.

Simpson-Vlach, R., \& Ellis, N. C. (2010). An Academic Formulas List: New Methods in Phraseology Research. Applied Linguistics, 31(4), 487-512. doi:10.1093/applin/amp058 
Strevens, P. (1973). Technical, technological, and scientific English. ELT Journal, 27, 223234.

stroke. (n.d.). In Merriam-Webster.com. Retrieved from July 14, 2017, https://www.merriam-webster.com/dictionary/stroke

Sutarsyah, C., Nation, P., \& Kennedy, G. (1994). How useful is EAP vocabulary for ESP? A corpus based case study. RELC Journal, 25(2), 34-50.

Taavitsainen, I. (2006). Medical communication: Lingua francas. In K. Brown (Ed.), Encyclopedia of language and linguistics (2 ed., pp. 643-644). Amsterdam: Elsevier.

Tiersma, P. M. (1999). Legal language. Chicago: University of Chicago Press.

Valipouri, L., \& Nassaji, H. (2013). A corpus-based study of academic vocabulary in chemistry research articles. Journal of English for Academic Purposes, 12(4), 248263. doi:10.1016/j.jeap.2013.07.001

Van Rijn-van Tongeren, G. (1997). Metaphor in medical texts. Amsterdam: Rodopi.

van Zeeland, H., \& Schmitt, N. (2013). Lexical coverage in L1 and L2 listening comprehension: The same or different from reading comprehension? Applied Linguistics, 34(4), 457-479. doi:10.1093/applin/ams074

Wang, J., Liang, S. L., \& Ge, G. C. (2008). Establishment of a Medical Academic Word List. English for Specific Purposes, 27(4), 442-458. doi:10.1016/j.esp.2008.05.003

Ward, J. (1999). How large a vocabulary do EAP engineering students need? Reading in a foreign language, 12(2), 309-323.

Ward, J. (2007). Collocation and technicality in EAP engineering. Journal of English for Academic Purposes, 6(1), 18-35. doi:10.1016/j.jeap.2006.10.001

Ward, J. (2009). A basic engineering English word list for less proficient foundation engineering undergraduates. English for Specific Purposes, 28(3), 170-182.

Ward, J., \& Chuenjundaeng, J. (2009). Suffix knowledge: Acquisition and applications. System, 37(3), 461-469. doi:10.1016/j.system.2009.01.004

Watson Todd, R. (2017). An opaque engineering word list: Which words should a teacher focus on? English for Specific Purposes, 45, 31-39. doi:10.1016/j.esp.2016.08.003

Webb, S., \& Chang, A. C.-S. (2012). Second language vocabulary growth. RELC Journal, 43(1), 113-126.

Webb, S., \& Rodgers, M. P. H. (2009a). The lexical coverage of movies. Applied Linguistics, 30(3), 407-427. doi:10.1093/applin/amp010 
Webb, S., \& Rodgers, M. P. H. (2009b). Vocabulary demands of television programs. Language Learning, 59(2), 335-366.

West, M. (1953). A general service list of English words. London: Longman.

Wette, R., \& Hawken, S. J. (2016). Measuring gains in an EMP course and the perspectives of language and medical educators as assessors. English for Specific Purposes, 42, 38-49. doi:10.1016/j.esp.2015.11.002

Williams, R. (1985). Teaching vocabulary recognition strategies in ESP reading. ESP Journal, 4, 121-131.

Wood, D. C., \& Appel, R. (2014). Multiword constructions in first year business and engineering university textbooks and EAP textbooks. Journal of English for Academic Purposes, 15, 1-13. doi:10.1016/j.jeap.2014.03.002

Woodward-Kron, R. (2008). More than just jargon - the nature and role of specialist language in learning disciplinary knowledge. Journal of English for Academic Purposes, 7(4), 234-249.

Wray, A. (2000). Formulaic sequences in second language teaching: Principle and practice. Applied Linguistics, 21(4), 463-489.

Wu, W., \& Hammond, M. (2011). Challenges of university adjustment in the UK: a study of East Asian Master's degree students. Journal of Further and Higher Education, 35(3), 423-438. doi:10.1080/0309877x.2011.569016

Xue, G., \& Nation, P. (1984). A university word list. Language Learning and Communication, $3(2), 215-229$.

yang. (n.d.). In Merriam-Webster.com. Retrieved from August 15, 2017, https://www.merriam-webster.com/dictionary/yang

Yang, H. (1986). A new technique for identifying scientific/technical terms and describing science texts (an interim report). Literary and Linguistic Computing, 1, 93-103.

Yang, M.-N. (2015). A nursing academic word list. English for Specific Purposes, 37, 27-38.

yin. (n.d.). In Merriam-Webster.com. Retrieved from April 21, 2017, https://www.merriam-webster.com/dictionary/yin

Yu, N. (2003). Metaphor, body, and culture: The Chinese understanding of gallbladder and courage. Metaphor and symbol, 18(1), 13-31. 


\section{Appendices}

\section{Appendix 1. Results of vocabulary burden analysis}

\begin{tabular}{|c|c|c|c|c|}
\hline BASEWRD & $\begin{array}{l}\text { TCM } \\
\text { Corpora }\end{array}$ & $\begin{array}{l}\text { Theory-based } \\
\text { textbooks }\end{array}$ & $\begin{array}{l}\text { Practice-based } \\
\text { textbooks }\end{array}$ & $\begin{array}{l}\text { Journal } \\
\text { articles }\end{array}$ \\
\hline Supplementary word lists & $6.12 \%$ & $5.15 \%$ & $7.65 \%$ & $6.84 \%$ \\
\hline TCM base word lists & $12.05 \%$ & $10.17 \%$ & $16.87 \%$ & $9.50 \%$ \\
\hline 1 & $68.16 \%$ & $69.12 \%$ & $70.91 \%$ & $64.75 \%$ \\
\hline 2 & $78.62 \%$ & $79.32 \%$ & $79.35 \%$ & $77.30 \%$ \\
\hline 3 & $87.36 \%$ & $86.89 \%$ & $86.15 \%$ & $89.00 \%$ \\
\hline 4 & $91.22 \%$ & $90.97 \%$ & $90.20 \%$ & $92.47 \%$ \\
\hline 5 & $93.25 \%$ & $93.27 \%$ & $92.51 \%$ & $93.97 \%$ \\
\hline 6 & $94.26 \%$ & $94.33 \%$ & $93.60 \%$ & $94.87 \%$ \\
\hline 7 & $95.05 \%$ & $95.02 \%$ & $94.36 \%$ & $95.78 \%$ \\
\hline 8 & $95.83 \%$ & $95.82 \%$ & $95.33 \%$ & $96.36 \%$ \\
\hline 9 & $96.50 \%$ & $96.43 \%$ & $95.93 \%$ & $97.15 \%$ \\
\hline 10 & $97.15 \%$ & $97.14 \%$ & $96.79 \%$ & $97.55 \%$ \\
\hline 11 & $97.62 \%$ & $97.68 \%$ & $97.30 \%$ & $97.91 \%$ \\
\hline 12 & $97.91 \%$ & $97.97 \%$ & $97.65 \%$ & $98.13 \%$ \\
\hline 13 & $98.36 \%$ & $98.37 \%$ & $98.25 \%$ & $98.49 \%$ \\
\hline 14 & $98.85 \%$ & $98.92 \%$ & $98.86 \%$ & $98.79 \%$ \\
\hline 15 & $98.99 \%$ & $99.06 \%$ & $98.96 \%$ & $98.97 \%$ \\
\hline 16 & $99.18 \%$ & $99.28 \%$ & $99.12 \%$ & $99.15 \%$ \\
\hline 17 & $99.36 \%$ & $99.42 \%$ & $99.31 \%$ & $99.35 \%$ \\
\hline 18 & $99.49 \%$ & $99.49 \%$ & $99.48 \%$ & $99.50 \%$ \\
\hline 19 & $99.69 \%$ & $99.73 \%$ & $99.71 \%$ & $99.64 \%$ \\
\hline 20 & $99.78 \%$ & $99.81 \%$ & $99.77 \%$ & $99.78 \%$ \\
\hline 21 & $99.83 \%$ & $99.85 \%$ & $99.81 \%$ & $99.85 \%$ \\
\hline 22 & $99.87 \%$ & $99.88 \%$ & $99.86 \%$ & $99.90 \%$ \\
\hline 23 & $99.94 \%$ & $99.96 \%$ & $99.93 \%$ & $99.95 \%$ \\
\hline 24 & $99.96 \%$ & $99.98 \%$ & $99.94 \%$ & $99.97 \%$ \\
\hline 25 & $100.00 \%$ & $100.00 \%$ & $100.00 \%$ & $100.00 \%$ \\
\hline
\end{tabular}




\section{Appendix 2. Sub-list 1 of the TCM technical word list at Level 1}

Note: The word types were arranged by their number of occurrences in the TCM Corpora. The concordance lines of word types falling into a single lemma (e.g., point, points) were checked to see if they had similar meanings and patterns of use in the TCM Corpora. If so, they were combined into a single entry in the word list as point(s).

\begin{tabular}{|c|c|c|c|}
\hline 1. blood & 26. fire & 51. treat(ed/ing/s) & 76. quality(ies) \\
\hline 2. treatment & 27. result & 52. $\operatorname{organ}(\mathrm{s})$ & 77. principle(s) \\
\hline 3. heat & 28. pattern(s) & 53. cell(s) & 78. indicate $(\mathrm{d} / \mathrm{s} / \mathrm{ing})$ \\
\hline 4. pain & 29. control(led/s) & 54. feeling & 79. traditional \\
\hline 5. use(d)/using & 30. case(s) & 55. factor (s) & 80. yellow \\
\hline 6. patient(s) & 31. chest & 56. compared & 81. activity/activities \\
\hline 7. group(s) & 32. clear(s/ing) & 57. participants & 82. disorder(s) \\
\hline 8. heart & 33. vessel(s) & 58. severe & 83. weak \\
\hline 9. cold & 34. $\operatorname{add}(\mathrm{ed})$ & 59. excess & 84. empty \\
\hline 10. medicine(s) & 35. related & 60. therapy/therapies & 85. associated \\
\hline 11. point(s) & 36. water & 61. medical & 86. shan \\
\hline 12. symptom(s) & 37. trail(s) & 62. significantly & 87. cough(ing) \\
\hline 13. study & 38. mind & 63. manifestation(s) & 88. model(s) \\
\hline 14. body & 39. red & 64. flow & 89. middle \\
\hline 15. wind & 40. data & 65. coating & 90. age \\
\hline 16. tongue & 41. method(s) & 66. health & 91. bleeding \\
\hline 17. stomach & 42. significant & 67. painful & 92. burner(s) \\
\hline 18. clinical & 43. function(s) & 68. system(s) & 93. practice \\
\hline 19. channel(s) & 44. western & 69. internal & 94. addition(s) \\
\hline 20. low(er) & 45. main & 70. food(s) & 95. primary \\
\hline 21. damp & 46. dry & 71. include(d/ing) & 96. throat \\
\hline 22. formula(s/e) & 47. skin & 72. women & 97. signs \\
\hline 23. $\operatorname{effect}(\mathrm{s})$ & 48. condition(s) & 73. depression & 98. excessive \\
\hline 24. disease(s) & 49. cause(s/d/ing) & 74. effective & 99. rat(s) \\
\hline 25. dampness & 50. level(s) & 75. normal & 100. area(s) \\
\hline
\end{tabular}


Continued

\begin{tabular}{|c|c|c|c|}
\hline 101. expression(s) & 126. fullness & 151. $\operatorname{action}(\mathrm{s})$ & 176. sleep \\
\hline 102. swelling & 127. deep & 152. mean & 177. spirit \\
\hline 103. full & 128. rapid & 153. lack & 178. theory \\
\hline 104. test(s) & 129. $\min$ & 154. relationship(s) & 179. major \\
\hline 105. sweat(ing/s) & 130. muscle(s) & 155. intervention(s) & 180. source \\
\hline 106. mental & 131. root & 156. transformation & 181. statistical \\
\hline 107. upper & 132. risk & 157. thirst & 182. stroke \\
\hline 108. brain & 133. problems & 158. development(s) & 183. energy(ies) \\
\hline 109. mouth & 134. external & 159. outcome(s) & 184. injury \\
\hline 110. powder & 135. criterion(a) & 160. resolve(s/d) & 185. modern \\
\hline 111. pale & 136. region & 161. invasion & 186. pregnancy \\
\hline 112. difference(s) & 137. cancer & 162. process(es) & 187. eye \\
\hline 113.increase(d/s/ing) & 138. physical & 163. neck & 188. affect(ed/s/ing) \\
\hline 114. location & 139. period(s) & 164. score(s) & 189. observed \\
\hline 115. stage(s) & 140. nature & 165.supplement(ing) & 190. characteristics \\
\hline 116. changes & 141. pressure & 166. soul & 191. ye \\
\hline 117. indication(s) & 142. form(s) & 167. hot & 192. cycle \\
\hline 118. emotional & 143. poor & 168. evidence & 193. stress \\
\hline 119. warm(ing) & 144. difficult & 169. connecting & 194. tissue(s) \\
\hline 120. movement & 145. stimulation & 170. strong & 195. ban \\
\hline 121. thin & 146. loss & 171. decrease(d/s) & 196. applied \\
\hline 122. combined & 147. lead(ing/s) & 172. protein(s) & 197. follow \\
\hline 123. descend(ing/s) & 148. interior & 173. $\operatorname{drug}(\mathrm{s})$ & 198. improvement(s) \\
\hline 124. rate & 149. performed & 174. similar & 199. response(s) \\
\hline 125. combination(s) & 150.practitioner(s) & 175. reduce(d/ing/s) & 200. drain(ing) \\
\hline
\end{tabular}


Continued

\begin{tabular}{|c|c|c|c|}
\hline 201. influence(s) & 226. size(s) & 251. damage(s) & 276. nose \\
\hline 202. weight & 227. mechanism(s) & 252. $\operatorname{leg}(\mathrm{s})$ & 277. experimental \\
\hline 203. frequent & 228. receive(d/ing/s) & 253. resulting & 278. illness \\
\hline 204. standard(s) & 229. shoulder & 254. rising & 279. provide(d/s) \\
\hline 205. anxiety & 230. intension & 255. aspect(s) & 280. infertility \\
\hline 206. diet & 231. governing & 256. moves & 281. conventional \\
\hline 207. dryness & 232. defensive & 257.randomiz(s)ed & 282. circulation \\
\hline 208. involved & 233. sea & 258. events & 283. joint(s) \\
\hline 209. mice & 234. infection(s) & 259. measure $(\mathrm{d} / \mathrm{s})$ & 284. stiffness \\
\hline 210. conducted & 235. taste & 260.preparation(s) & 285. current \\
\hline 211. positive & 236. value(s) & 261. sexual & 286. sudden \\
\hline 212. transform(ing) & 237. frequency(ies) & 262. bone(s) & 287. obtained \\
\hline 213. surface & 238.promote(s/ing) & 263. memory & 288. strengthen(s) \\
\hline 214. benefit(s) & 239.regulate(s/ing/d) & 264. ear(s) & 289. active \\
\hline 215. inability & 240. vision & 265. position & 290. thick \\
\hline 216. phase(s) & 241. knee(s) & 266. exercise & 291. eliminate(s/ed) \\
\hline 217. hospital(s) & 242. difficulty & 267.identify(ied/ing) & 292. heaviness \\
\hline 218. sample(s) & 243. eating & 268. scale(s) & 293. functional \\
\hline 219. subjects & 244. original & 269. variation & 294. tiredness \\
\hline 220. potential & 245. weakness & 270. animal(s) & 295. spiritual \\
\hline 221. temperature & 246. bitter & 271. swollen & 296. breath(ing) \\
\hline 222. dose (s) & 247. comparison(s) & 272. individual & 297. develop(ed) \\
\hline 223.improve(d/ing/s) & 248. characteriz(s)ed & 273. occur(s) & 298. breast \\
\hline 224. arm & 249. ability & 274. $\operatorname{calm}(\mathrm{s})$ & 299. loose \\
\hline 225. healthy & 250. discharge(s) & 275. rebellious & 300.demonstrate(d/s) \\
\hline
\end{tabular}


Continued

\begin{tabular}{|c|c|c|c|}
\hline 301. strength(s) & 326. remedy & 351. negative & 376. scientific \\
\hline 302. approach & 327. basis & 352. presence & 377. enters(ing) \\
\hline 303. injection(s) & 328. growth & 353. element(s) & 378. colour \\
\hline 304. doctors & 329. safety & 354. surgery & 379. dependent \\
\hline 305. anger & 330. fur & 355. modifications & 380. release(s) \\
\hline 306. assess(ed/ing) & 331. breathlessness & 356. uncooked & 381. gene(s) \\
\hline 307. located & 332. strategies & 357. complex & 382. mainly \\
\hline 308. defined & 333. direct(ing) & 358. identification & 383. produce $(d)$ \\
\hline 309. mild & 334. provide(d/s) & 359. restore(s/ing) & 384. significance \\
\hline 310. oral & 335. consumption & 360. tip & 385. underlying \\
\hline 311. ingredients & 336. daily & 361. failure & 386. centre \\
\hline 312. reduction & 337. alternative & 362. products & 387. limited \\
\hline 313. desire & 338. purple & 363. formation & 388. suffering \\
\hline 314. average & 339. nerve(s) & 364. sang & 389. concentration \\
\hline 315. floating & 340. irregular & 365. female(s) & 390. drink \\
\hline 316. prevent(s) & 341. effectiveness & 366. divided & 391. absence \\
\hline 317. balance & 342. intensity & 367. experienced & 392. network \\
\hline 318. directly & 343. relatively & 368. collected & 393. redness \\
\hline 319. evaluation & 344. restlessness & 369. immune & 394. opens \\
\hline 320. stops & 345. evaluate(ing) & 370. male(s) & 395. shortness \\
\hline 321. production & 346. objective & 371. determine(d) & 396. management \\
\hline 322. reinforcing & 347. central & 372. examination & 397. presented \\
\hline 323. application & 348. emotion(s) & 373. relieve & 398. branch \\
\hline 324. accompanied & 349. useful & 374. heaven & 399. marked \\
\hline 325. administration & 350. index & 375. relevant & 400. specifically \\
\hline
\end{tabular}


Continued

\begin{tabular}{|c|c|c|c|}
\hline 401. population & 426. chills & 451. helps & 476. procedure(s) \\
\hline 402. origin & 427. classical & 452. establish(ed) & 477. repeated \\
\hline 403. pronounced & 428. inner & 453. regulation & 478. protective \\
\hline 404. expressed & 429. degree & 454. masses & 479. sequence \\
\hline 405. sadness & 430. randomly & 455. suitable & 480. manifest \\
\hline 406. conception & 431. technique & $\begin{array}{l}456 . \\
\text { observation(s) }\end{array}$ & 481. complications \\
\hline 407. constitution & 432. variable(s) & 457. depth & 482. relation \\
\hline 408. random & 433. excluded & 458. examine(d) & 483. users \\
\hline 409. severity & 434. proper & 459. transporting & 484. agents \\
\hline 410. ache(s) & 435. smooth & 460. medicinal & 485. linking \\
\hline 411. moderate & 436. modified & 461. insufficient & 486. correspond(s) \\
\hline 412. containing & 437. core & 462. responsible & 487.measurement(s) \\
\hline 413. burning & 438. fried & 463. birth & 488. psychological \\
\hline 414. extract(s/ed) & 439. status & 464. correlation(s) & 489. reaction \\
\hline 415. alcohol & 440. gate & 465. consciousness & 490. substances \\
\hline 416. selection & $\begin{array}{l}441 . \\
\text { understanding }\end{array}$ & 466. corresponding & 491. sensitivity \\
\hline 417. mental & 442. chemical & 467. prevention & 492. confirmed \\
\hline 418. aged & 443. distribution & 468. revealed & 493. extreme \\
\hline 419. importance & 444. downward(s) & 469. nervous & 494. blinding \\
\hline 420. compounds & 445. $\operatorname{clinic}(\mathrm{s})$ & 470. stepping & 495. contrast \\
\hline 421. extraordinary & 446. dosage(s) & 471. chosen & 496. investigate(d) \\
\hline 422. visual & 447. fright & 472. recovery & 497. sore \\
\hline 423. carried & 448. signaling & 473. exhaustion & 498. consistent \\
\hline 424. components & 449. unable & 474. structure(s) & 499. superior \\
\hline 425. relative & 450. reflect(s/ing) & 475. fixed & 500. typical \\
\hline
\end{tabular}

Continued

\begin{tabular}{|l|l|l|l|}
\hline 501. clinically & 503. guidelines & 505. interaction(s) & 507. tendency \\
\hline 502. biological & 504. wasting & 506. initial & \\
\hline
\end{tabular}




\section{Appendix 3. Sub-list 1 of the TCM technical word list at Level 2}

\begin{tabular}{|c|c|c|c|}
\hline 1. yin & 26. tonify(ies/ing) & 51. induced & 76. hypertension \\
\hline 2. liver & 27. exterior & 52. needling & 77. therapeutic \\
\hline 3. deficiency(ies) & 28. urine & 53. moxibustion & 78. mucus \\
\hline 4. acupuncture & 29. ling & 54. cortex & 79. tian \\
\hline 5. radix & 30. obstruction(s) & 55. $\operatorname{limb}(\mathrm{s})$ & 80. retention \\
\hline 6. kidney(s) & 31. intestine(s) & 56. herba & 81. serum \\
\hline 7. spleen & 32. prescription(s) & 57. moxa & 82. asthma \\
\hline 8. phlegm & 33. deficient & 58. sensation(s) & 83. placebo \\
\hline 9. pulse(s) & 34. essence(s) & 59. constipation & 84. fang \\
\hline 10. decoction(s) & 35. acute & 60. efficacy & 85. distension \\
\hline 11. lung(s) & 36. diagnosis & 61. stool(s) & 86. sticky \\
\hline 12. herb(s) & 37. fever & 62. uterus & 87. wiry \\
\hline 13. syndrome(s) & 38. urination & 63. nausea & 88. diagnostic \\
\hline 14. tang & 39. pill(s) & 64. penetrating & 89. fatigue \\
\hline 15. rhizoma & 40. nourish(es/ing) & 65. lateral & 90. rehmanniae \\
\hline 16. chronic & 41. semen & 66. insomnia & 91. distention \\
\hline 17. stagnation & 42. urinary & 67. acupoint(s) & 92. pericardium \\
\hline 18. fluid(s) & 43. vomiting & 68. glucose & 93. headaches \\
\hline 19. fu & 44. dizziness & 69. pathology(ies) & 94. tinnitus \\
\hline 20. herbal & 45. gallbladder & 70. adverse & 95. expel(s) \\
\hline 21. stasis & 46. wan & 71. pathological & 96. diarrh(o)ea \\
\hline 22. fructus & 47. diabetes & 72. vacuity & 97. yuan \\
\hline 23. abdominal & 48. needle(s) & 73. differentiation & 98. acupressure \\
\hline 24. bladder & 49. dang & 74. dong & 99. scanty \\
\hline 25. pathogenic & 50. abdomen & 75. sham & 100. slippery \\
\hline
\end{tabular}


Continued

\begin{tabular}{|c|c|c|c|}
\hline 101. sclerotium & 126. aversion & 151. protocol(s) & 176. nutritive \\
\hline 102. duration & 127. sinew(s) & 152. upward(s) & 177. metabolism \\
\hline 103. menstrual & 128. paeoniae & 153. marrow & 178. ramulus \\
\hline 104. $\operatorname{acid}(\mathrm{s})$ & 129. insulin & 154. activation & 179. blurred \\
\hline 105. glycyrrhizae & 130. administered & 155. nasal & 180. cerebral \\
\hline 106. appetite & 131. medicinals & 156. physiological & 181. wheezing \\
\hline 107. lumbar & 132. uralensis & 157. ovulation & 182. febrile \\
\hline 108. disharmony(ies) & 133. greasy & 158. chemotherapy & 183. medication(s) \\
\hline 109. angelicae & 134. orifice(s) & 159. diagnosed & 184. seu \\
\hline 110. cocos & 135. diabetic & 160. spine & 185. apoptosis \\
\hline 111. officinalis & 136. flos & 161. sperm & 186. cinnamomi \\
\hline 112. chinensis & 137. inflammatory & 162. subtractions & 187. elbow \\
\hline 113. poriae & 138. turbid & 163. agitation & 188. contraction \\
\hline 114. citri & 139. backache & 164. astragali & 189. uterine \\
\hline 115. oedema & 140. menstruation & 165. bi & 190. inclusion \\
\hline 116. distil & 141. onset & 166. prescribe(d) & 191.invigorate(s/ing) \\
\hline 117. palpitation(s) & 142. dysfunction & 167.macrocephalae & 192. itching \\
\hline 118. complexion & 143. tong & 168. oppression & 193. needled \\
\hline 119. atractylodis & 144. chai & 169. physician(s) & 194. anterior \\
\hline 120. numbness & 145. gen & 170. pericarpium & 195. parameters \\
\hline 121. median(s) & 146. inflammation & 171. ascend(s) & 196. dull \\
\hline 122. ethereal & 147. massage & 172. pathogen(s) & 197. respiratory \\
\hline 123. sinensis & 148. irritability & 173. pathways & 198. praeparata \\
\hline 124. oedema & 149. bowel(s) & 174. epilepsy & 199. toxicity \\
\hline 125. ginseng & 150. ascending & 175. tuber & 200. heterogeneity \\
\hline
\end{tabular}


Continued

\begin{tabular}{|l|l|l|l|}
\hline 201. profuse & 218. reticulatae & 235. renal & 252. moutan \\
\hline 202.complementary & 219. toxic & 236. cardiac & 253. upright \\
\hline 203. toxin(s) & 220. cassiae & 237. corporeal & 254. oblique \\
\hline 204. superficial & 221. ophiopogonis & 238. incidence & 255. fibrosis \\
\hline 205. medication & 222. miscarriage(s) & 239. disperse(s) & 256. japonici \\
\hline 206. obstructing & 223. midline & 240. subdues & 257. pinelliae \\
\hline 207. costal & 224. tonification & 241. gong & 258. intestinal \\
\hline 208. radicis & 225. perpendicular & 242.harmonize(s/ing) & 259. lactiflorae \\
\hline 209. deviation & 226. stagnant & 243. intake & 260. obstructed \\
\hline 210. aetiology & 227. diaphragm & 244. allergic & 261.miltiorrhizae \\
\hline 211. epigastrium & 228. discomfort & 245. oppositae & 262. impairment \\
\hline 212. spinal & 229. poria & 246. auricular & 263. wrist \\
\hline 213. artery(ies) & 230. atrophy & 247.gastrointestinal & \\
\hline 214. tumor(s) & 231.acupuncturist(s) & 248. glutinosae & \\
\hline 215. caulis & 232. dampness & 249. proliferation & \\
\hline 216. beneficial & 233. dioscoreae & 250. spontaneous & \\
\hline 217. facial & 234. medial & 251. fen & \\
\hline
\end{tabular}




\section{Appendix 4. Sub-list 1 of the TCM technical word list at Level 3}

\begin{tabular}{|c|c|c|c|}
\hline 1. qi & 18. sanyinjiao & 35. sha & 52. lieque \\
\hline 2. yang & 19. zusanli & 36. gou & 53. qihai \\
\hline 3. $\mathrm{zi}$ & 20. xiao & 37. luo & 54. zhishi \\
\hline 4. shen & 21. xie & 38. xiong & 55. cang \\
\hline 5. zhi & 22. xia & 39. xuan & 56. baihui \\
\hline 6. cao & 23. zhong & 40. neiguan & 57. yanglingquan \\
\hline 7. pi & 24. zao & 41. taixi & 58. gua \\
\hline 8. jiao & 25. qin & 42. po & 59. hou \\
\hline 9. $\mathrm{mu}$ & 26. huo & 43. quchi & 60. dou \\
\hline 10. shi & 27. hegu & 44. niu & 61. qiao \\
\hline 11. sheng & 28. shenshu & 45. pishu & 62. xian \\
\hline 12. jing & 29. jie & 46. zhen & 63. zhaohai \\
\hline 13. ji & 30. zheng & 47. xue & 64. fengchi \\
\hline 14. shu & 31. xing & 48. xin & 65. zangfu \\
\hline 15. cun & 32. taichong & 49. rou & 66. fenglong \\
\hline 16. xiang & 33. guanyuan & 50. zang & 67. feishu \\
\hline 17. lian & 34. zhongwan & 51. yinlingquan & 68. zhongji \\
\hline
\end{tabular}




\section{Appendix 5. Sub-list 2 of the TCM technical word list at Level 1}

\begin{tabular}{|c|c|c|c|}
\hline 1. detected & 26. stimulate(s/d) & 51. soreness & 76. imbalance(s) \\
\hline 2. survival & 27. limitation(s) & 52. detection & 77. continuous \\
\hline 3. difficulties & 28. increases & 53. locate & 78. methodological \\
\hline 4. independent & 29. internally & 54. tension & 79. portion \\
\hline 5. laboratory & 30. presentation & 55. occur(red/ring) & 80. complicated \\
\hline 6. techniques & 31. articles & 56. formed & 81. infant(s) \\
\hline 7. exposure & 32. $\operatorname{contain}(\mathrm{s} / \mathrm{ed})$ & 57. dietary & 82. intense \\
\hline 8. ancient & 33. tested & 58. functioning & 83. signal \\
\hline 9. allocation & 34. context & 59. movements & 84. randomization \\
\hline 10. childhood & 35. correct & 60. environment & 85. substance \\
\hline 11. flows & 36. unclear & 61. extremities & 86. achieve(d) \\
\hline 12. modification & 37. experiment(s) & 62. sessions & 87. enter \\
\hline 13. passage(s) & 38.manifest(s/ing/ed) & 63. referred & 88. transform(ed/s) \\
\hline 14. requires & 39. resistance & 64. supplements & 89. positions \\
\hline 15. variety & 40. transportation & 65.derive(d/ing/s) & 90. perspective(s) \\
\hline 16. attacks & 41. individuals & 66. fall(s/ing) & 91. practices \\
\hline 17. properties & 42. solution & 67. gathering & 92. regions \\
\hline 18. reactions & 43. variations & 68. illnesses & 93. describe \\
\hline 19. characteristic & 44. depend(s/ing) & 69. integrated & 94. shock \\
\hline 20. connect(s/ed) & 45. suffer(ed/s) & 70. minor & 95. appearance \\
\hline 21. pregnant & 46. adopted & 71. simultaneously & 96. develop(ing/s) \\
\hline 22. normally & 47. stiff & 72. density & 97. stronger \\
\hline $\begin{array}{l}\text { 23.generate(d/in } \\
\text { g) }\end{array}$ & 48. sinking & 73. concept(s) & 98. compound \\
\hline 24. component & 49. watery & 74. fertility & 99. elderly \\
\hline 25. content(s) & 50. participant & 75. capacity & 100. binding \\
\hline
\end{tabular}


Continued

\begin{tabular}{|c|c|c|c|}
\hline 101. cycles & 126. employed & 151. reflected & 176. approaches \\
\hline 102. electrical & 127. produce(s/ing) & 152. stored & 177. prevent(ing/s) \\
\hline 103. nan & 128. quantitative & 153. behaviour & 178. counter \\
\hline 104. contribute(s) & 129. enhance(d) & 154. elevated & 179. concentrations \\
\hline 105. coupled & 130. manual & 155. manner & 180. $\operatorname{cool}(\mathrm{s} / \mathrm{ing})$ \\
\hline 106. similarity & 131. inserted & 156. peak & 181. distinguish \\
\hline 107. labour & 132. removes & 157. resulted & 182. markedly \\
\hline 108. manipulation & 133. assigned & 158. sufficient & 183. strain \\
\hline 109. $\operatorname{aim}(\mathrm{s} / \mathrm{ed})$ & 134. compare(ing) & 159. blocked & 184. aching \\
\hline 110. controlling & 135. effectively & 160. depressive & 185. drains \\
\hline 111. recruited & 136. situations & 161. examinations & 186. principal \\
\hline 112. boost(s) & 137. vital & 162. substantial & 187. invasions \\
\hline 113.eliminating & 138. thirsting & 163. ingredient & 188. stimulate(ing) \\
\hline 114. extraction & 139. blind & 164. strengthening & 189. constant \\
\hline 115. progression & 140. explore(d) & 165. cure (d) & 190. healing \\
\hline 116. tube(s) & 141. linked & 166. percentage & 191. outer \\
\hline 117. virus(es) & 142. persistent & 167. resolving & 192. remedies \\
\hline 118. exercises & 143. blockage & 168. disturbance(s) & 193. obtain \\
\hline 119. raw & 144. curative & 169. angle & 194. warmth \\
\hline 120. stable & 145. motor & 170. gender & 195. damaged \\
\hline 121. accuracy & 146. transport & 171.maintain(ed/ing) & 196. exhibited \\
\hline 122. emission & 147. calming & 172. composed & 197. fundamental \\
\hline 123. experiences & 148. collapse & 173. relieve(ing/s/d) & 198. strain(ing/ed) \\
\hline 124. involve(s/ing) & 149. injure(s) & 174. energetic & 199. bacterial \\
\hline 125. molecular & 150. produces & 175. present(s) & 200. evil(s) \\
\hline
\end{tabular}


Continued

\begin{tabular}{|c|c|c|c|}
\hline 201. participate(ing) & 226.correspodence(s) & 251. profiles & 276. mode \\
\hline 202. restless & 227. inadequate & 252. separated & 277. preference \\
\hline 203. variance & 228. occurrence & 253. statistics & 278. quantity \\
\hline $\begin{array}{l}\text { 204.accumulate(d/s } \\
\text { /ing) }\end{array}$ & 229. standardized & 254. chemicals & 279. cloudy \\
\hline 205. mortality & 230. arise(s) & 255. reaches & 280. depressed \\
\hline 206. reverse & 231. simultaneous & 256. stones & 281. presenting \\
\hline 207. regulatory & 232. tenderness & 257.accompanying & 282. psychiatric \\
\hline 208. emphasis & 233. proportion & 258. allocated & 283. toes \\
\hline 209. injected & 234. flowing & 259. equivalent & 284. generalized \\
\hline 210. adjusted & 235. assist(ed) & 260. warms & 285. weaken(s) \\
\hline 211. disturbed & 236. removing & 261. balanced & 286. definition \\
\hline 212. rapidly & 237. fatty & 262. representative & 287.prescription(s) \\
\hline 213. constitutional & 238. focuses & 263. rises & 288. sores \\
\hline 214. theories & 239. helpful & 264. scatter(s/ing) & 289. consequence \\
\hline 215. validity & 240. unblock & 265. perceived & 290. implies \\
\hline 216. decline & 241. hardness & 266. successfully & 291. paired \\
\hline 217. blind & 242. integration & 267. уа & 292. radiating \\
\hline 218. correlated & 243. collecting & 268. attributed & 293. screening \\
\hline 219. orally & 244. excellent & 269. frustration & 294. subgroup \\
\hline 220. tightness & 245. marker(s) & 270. confusion & 295. respective \\
\hline 221. courses & 246. influenced & 271. thickness & 296. sighing \\
\hline 222. stopping & 247. separation & 272. expert & 297. damaging \\
\hline 223. quicken(s) & 248. bacteria & 273. sided & 298. differ(ed) \\
\hline 224. treasures & 249. presents & 274. distinct & 299. distributed \\
\hline 225. respondents & 250. involvement & 275. fertile & 300. oils \\
\hline
\end{tabular}


Continued

\begin{tabular}{|l|l|l|l|}
\hline 301. rebellion & 321. imaging & 341. symptomatic & 361. habits \\
\hline 302. reinforce(d) & 322. incomplete & 342. taxation & 362. combines \\
\hline 303. varied & 323. specificity & 343. emptiness & 363. decreasing \\
\hline 304. benefiting & 324. sympathetic & 344. participation & 364. disruption \\
\hline 305. lifting & 325. circulate(s/ing) & 345. bluish & 365. palms \\
\hline 306. disability & 326. interpreted & 346. lowering & 366. behavioral \\
\hline 307. drying & 327. pretreatment & 347. determination & 367. comparable \\
\hline 308. flavors & 328. classics & 348. discrimination & 368. disc \\
\hline 309. mouse & 329. mechanical & 349. guiding & 369. inappropriate \\
\hline 310. retained & 330. observe & 350. relaxation & 370. parallel \\
\hline 311. curve & 331. formulation(s) & 351. cools & 371. situated \\
\hline 312. hypothesis & 332. heavenly & 352. selective & 372. transferred \\
\hline 313. radical & 333. indicator(s) & 353. vary(ies) & 373. withdrawal \\
\hline 314. recruitment & 334. stained & 354. combining & 374. consultation \\
\hline 315. coldness & 335. delayed & 355. instrument(s) & 375. evident \\
\hline 316. documented & 336. processed & 356. integrative & 376. indices \\
\hline 317. emerges & 337. steaming & 357. partial & 377. infectious \\
\hline 318. processing & 338. frontal & 358. reactive & 378. measuring \\
\hline 319. confirm(s) & 339. isolated & 359. acquisition & 379.phenomenon(a) \\
\hline 320. govern(s) & 340. partially & 360. cultured & 380. spreads \\
\hline
\end{tabular}




\section{Appendix 6. Sub-list 2 of the TCM technical word list at Level 2}

\begin{tabular}{|c|c|c|c|}
\hline 1. salviae & 26. trichosanthis & 51.electroacupuncture & 76. mania \\
\hline 2. wen & 27. dementia & 52. lycii & 77. pharmacological \\
\hline 3. $\tan$ & 28. lipid(s) & 53. palpation & 78. amenorrhoea \\
\hline 4. zingiberis & 29. sour & 54. pungent & 79. fasting \\
\hline 5. importance & 30. cognitive & 55. incontinence & 80. genital(s) \\
\hline 6. induce(s) & 31. sputum & 56. metabolic & 81. hepatitis \\
\hline 7. membranacei & 32. arthritis & 57. exiting & 82. pivot \\
\hline 8. abnormal & 33. hui & 58. manic & 83. subjective \\
\hline 9. cardiovascular & 34. paralysis & 59. rhinitis & 84. ternatae \\
\hline 10. patent & 35. coronary & 60. cytokine(s) & 85. motility \\
\hline 11. tract & 36. saline & 61. bile & 86. antibiotics \\
\hline 12. receptor(s) & 37. vascular & 62. prolapse & 87. granule(s) \\
\hline 13. impaired & 38. classified & 63. jaundice & 88. obstruct(s) \\
\hline 14. alismatis & 39. peripheral & 64. obesity & 89. prolonged \\
\hline 15. vertigo & 40. rehmannia & 65. ischemic & 90. hao \\
\hline 16. inhibition & 41. expel(ling/led/s) & 66. ligustici & 91. applicable \\
\hline 17. membrane(s) & 42. pulmonary & 67. median & 92. coptidis \\
\hline 18. vaginal & 43. cavity(ies) & 68. schisandrae & 93. invading \\
\hline 19. digestive & 44. collaterals & 69. exogenous & 94. lesion(s) \\
\hline 20. enuresis & 45. posterior & 70. recurrent & 95. divergent \\
\hline 21. cholesterol & 46. subdue(s) & 71. caution & 96. viral \\
\hline 22. concomitant & 47. turbidity & 72. neurons & 97. alleviate(s/d) \\
\hline 23. prevalence & 48. ulcer(s) & 73. angina & 98. antibody(ies) \\
\hline 24.activate(d/s/ing) & 49. corni & 74. myocardial & 99. ischemia \\
\hline 25. tendon(s) & 50. scutellariae & 75. pruni & 100.itching \\
\hline
\end{tabular}


Continued

\begin{tabular}{|c|c|c|c|}
\hline 101. wallichii & 126. $\operatorname{spasm}(\mathrm{s})$ & 151. nocturnal & 176. empirical \\
\hline 102. choppy & 127. residual & 152. eczema & 177. prone \\
\hline 103.inhibit(ed/ing/s) & 128. fetus & 153. seminal & 178. wristbands \\
\hline 104. analgesic & 129. diameter & 154. carthami & 179. tremor(s) \\
\hline 105. nourishment & 130. accordance & 155. abscess & 180. aggravate(d) \\
\hline 106. phellodendri & 131. hormone(s) & 156. moisten(s/ing) & 181. anus \\
\hline 107. saliva & 132. malaria & 157. p53 & 182. diagnose(s/d) \\
\hline 108. umbilicus & 133. secretion(s) & 158. premature & 183. magnoliae \\
\hline 109. cum & 134. forhead & 159. penetrate(s) & 184. analgesia \\
\hline 110. insufficiency & 35. rhei & 160. scrophulariae & 185. neurological \\
\hline 111. kit & 36. curcumae & 161. ziziphi & 186. platelet \\
\hline 112. overwork & 37. defeaction & 162. polygoni & 187. sensory \\
\hline 113. infarction & 138. intercostal & 163. coicis & 188. grief \\
\hline 114. incubated & 139. proximal & 164. predominant & 189. systemic \\
\hline 115. prostate & 140. threshold(s) & 165. biochemical & 190. abnormalities \\
\hline 116. antioxidant & 141.antiinflammatory & 166. matrix & 191. endometriosis \\
\hline 117. dispersing & 142. gall & 167. ding & 192. trauma \\
\hline 118. fibromyalgia & 143. kirlowii & 168. extinguish(es) & 193. jasminoidis \\
\hline 119. atractylodes & 144. excretion & 169. gastric & 194. caspase \\
\hline 120. cornu & 145.inferior & 170. recurrence & 195. implantation \\
\hline 121. psoriasis & 146. gypsum & 171. codonopsis & 196. buffer \\
\hline 122. embryo(s) & 147. physiology & 172. qualitative & 197. cellular \\
\hline 123. gardeniae & 148. tonic(s) & 173. oxidative & 198. colon \\
\hline 124. plasma & 149. pilosulae & 174. recens & 199. enrich(es) \\
\hline 125. propensity & 150. ankle & 175. transverse & 200. inhibitory \\
\hline
\end{tabular}


Continued

\begin{tabular}{|c|c|c|c|}
\hline 201. rheumatoid & 226. carmichaeli & 251. Bcl2 & 276. $\operatorname{decoct}(\mathrm{ed})$ \\
\hline 202. concealment & 227. sprain & 252. perimenstrual & 277. eligible \\
\hline 203. endothelial & 228. grind & 253. demographic & 278. eucommiae \\
\hline 204. ningpoensis & 229. pancreatic & 254. fibroids & 279. minimal \\
\hline 205. pediatric & 230. ping & 255. interval(s) & 280. contraindicated \\
\hline 206. preproductive & 231. mellitus & 256. knotted & 281. harmony \\
\hline 207. hippocampus & 232. RNA & 257. luteal & 282. hyperactivity \\
\hline 208. hypochondrium & 233. sneezing & 258. pituitary & 283. invade(s/d) \\
\hline 209. osteoarthritis & 234. achyranthis & 259. tinctorii & 284. prostatitis \\
\hline 210. persicae & 235. coefficient & 260. astragalus & 285. cervi \\
\hline 211. pus & 236. originate(s) & 261. descent & 286. postoperative \\
\hline 212. regurgitation & 237. undigested & 262. ode & 287. perparata \\
\hline 213. stabbing & 238. amomi & 263. ovary(ies) & 288. sedate \\
\hline 214. haemorrhoids & 239. carcinoma & 264. rigidity & 289. enzyme(s) \\
\hline 215. occipital & 240. hypertensive & 265. tenuifoliae & 290. induction \\
\hline 216. overflowing & 241. moist & 266. tingling & 291. lassitude \\
\hline 217. synthesis & 242. neuropathy & 267. diminished & 292. mitochondrial \\
\hline 218. focal & 243. vulgaris & 268. immaturus & 293. spinosae \\
\hline 219. gums & 244. arterial & 269. thready & 294. cirrhosis \\
\hline 220. influenza & 245. armeniacae & 270. transcription & 295. depletion \\
\hline 221. jujubae & 246. bidentatae & 271. unilateral & $\begin{array}{l}\text { 296.differentiate(d/ } \\
\text { ing) }\end{array}$ \\
\hline 222. progestrone & 247. blot & 272. convulsions & 297. endogenous \\
\hline 223. vivo & 248. carotid & 273. thigh & 298. rash(es) \\
\hline 224. axilla & 249. cyrus & 274. Ccl4 & 299. systolic \\
\hline 225. biomedical & 250. latent & 275. copious & 300. colic \\
\hline
\end{tabular}


Continued

\begin{tabular}{|c|c|c|c|}
\hline 301. dribbling & 325. canthus & 349. engender(s) & 373. scrofula \\
\hline 302. inhibitor(s) & 326. cyperi & 350. thermal & 374. surgical \\
\hline 303. coptis & 327. hypogastric & 351. adjacent & 375. ting \\
\hline 304. distending & 328. hypoglycemic & 352. atherosclerosis & 376. ulmoidis \\
\hline 305. invigorating & 329. $\mathrm{mmHg}$ & 353. bulbus & 377. amplitude \\
\hline 306. scapula & 330. opioid & 354. latency & 378. crude \\
\hline 307. jobi & 331. tablet(s) & 355. platycoid & 379. exertion \\
\hline 308. precursor(s) & 332. collagen & 356. vaccine & 380. fragrant \\
\hline 309. coma & 333. plantaginis & 357. capsule(s) & 381. horizontal \\
\hline 310. irritable & 334. rotation & 358. endometrial & 382. japonicae \\
\hline 311. laser & 335. soggy & 359. gynaecological & 383. puncture \\
\hline 312. migraine & 336. terminal & 360. mast & 384. cleft \\
\hline 313. pancreas & 337. ventricular & 361. oriental & 385. epithelial \\
\hline 314. pensiveness & 338. anatomical & 362. puerariae & 386. fibrosum \\
\hline 315. pores & 339. aspheloidis & 363.antihypertensive & 387. fluoxetine \\
\hline 316. aggregation & 340. gel & 364. multiflori & 388. hippocampal \\
\hline 317. girdle & 341. radial & 365. obese & 389. notoginseng \\
\hline 318. intermittent & 342. modalities & 366. delirium & 390. placenta \\
\hline 319. rehabilitation & 343. muscular & 367. diffuse(ing) & 391. simulation \\
\hline 320. sciatica & 344. ostreae & 368. leucorrhoea & 392. tolerance \\
\hline 321. consolidate & 345. pneumonia & 369. mediators & 393. foul \\
\hline 322. lachryma & 346. upregulated & 370. necrosis & 394. haemorrhage \\
\hline 323. pelvic & 347. autonomic & 371. nephropathy & 395. pernicious \\
\hline 324. vertebra€ & 348. emaciation & 372. polygalae & \\
\hline
\end{tabular}


Appendix 7. Sub-list 2 of the TCM technical word list at Level 3

\begin{tabular}{|c|c|c|c|}
\hline 1. geng & 18. ququan & 35. zhigou & 52. qiang \\
\hline 2. bei & 19. geshu & 36. zhe & 53. zulinqi \\
\hline 3. xuehai & 20. xinshu & 37. qigong & 54. lue \\
\hline 4. waiguan & 21. suo & 38. zha & 55. jianyu \\
\hline 5. mingmen & 22. weizhong & 39. tianshu & 56. shenting \\
\hline 6. deqi & 23. gongsun & 40. jue & 57. tongli \\
\hline 7. bian & 24. xingjian & 41. duan & 58. huantiao \\
\hline 8. shenmen & 25. ganshu & 42. jiu & 59. pangguangshu \\
\hline 9. yue & 26. sanjiaoshu & 43. taiyang & 60. shuidao \\
\hline 10. shou & 27. taichi & 44. zong & 61. neiting \\
\hline 11. sanjiao & 28. shenmai & 45. qimen & 62. huan \\
\hline 12. shuifen & 29. weishu & 46. taiyuan & 63. jiuwei \\
\hline 13. suan & 30. fengfu & 47. pian & 64. tiao \\
\hline 14. dazhui & 31. kunlun & 48. renzhong & 65. danggui \\
\hline 15. lun & 32. shanzhong & 49. taibai & 66. shangjuxu \\
\hline 16. fuliu & 33. rong & 50. yangming & 67. rangu \\
\hline 17. houxi & 34. daling & 51. chize & \\
\hline
\end{tabular}




\section{Appendix 8. Sub-list 3 of the TCM technical word list at Level 1}

\begin{tabular}{|c|c|c|c|}
\hline 1. constitute & 26. sciences & 51. generates & 76. immunity \\
\hline 2. redder & 27. varying & 52. tidal & 77. positively \\
\hline 3. urgency & 28. climatic & 53. virtue & 78. preventative \\
\hline 4. coping & 29. dreaming & 54. externally & 79. altered \\
\hline 5. molecules & 30. ineffective & 55. fats & 80. construct \\
\hline 6. concentrated & 31. interiorly & 56. radiates & 81. energizer \\
\hline 7. connective & 32.recommendations & 57. heating & 82. predictive \\
\hline 8. diseased & 33. separates & 58. progressive & 83. urgent \\
\hline 9. extending & 34. bodily & 59. theoretical & 84. advantages \\
\hline 10. lumps & 35. constituents & 60. untreated & 85. arising \\
\hline 11. professionals & 36. derive & 61. antidepressant & 86. completion \\
\hline 12. balancing & 37. determining & 62. evidenced & 87. dominant \\
\hline 13. cupping & 38. relies & 63. gentle & 88. enhancing \\
\hline 14. posttreatment & 39. travels & 64. indicative & 89. episodes \\
\hline 15. translate $(\mathrm{d})$ & 40. applying & 65. lacking & 90. implications \\
\hline 16. nonspecific & 41. binds & 66. removal & 91. radicals \\
\hline 17. probability & 42. gradual & 67. supportive & 92. shivering \\
\hline 18. runny & 43. reddish & 68. swallowing & 93. intensive \\
\hline 19. assessments & 44. structural & 69. complaint & 94. participating \\
\hline 20. facilitate & 45. conducting & 70. formulated & 95. poststroke \\
\hline 21. monitored & 46. inspection & 71. intention & 96. prospective \\
\hline 22. mutually & 47. transmitted & 72. consumed & 97. selecting \\
\hline 23. phenomenon & 48. variability & 73. developmental & 98. correlated \\
\hline 24. settles & 49. composition & 74. continuously & 99. undergoing \\
\hline 25. rabbit & 50. extending & 75. clinicians & 100. dries \\
\hline
\end{tabular}


Continued

\begin{tabular}{|l|l|l|l|}
\hline 101. migration & 20. screened & 139. contracted & 158. accurately \\
\hline 102. relaxes & 121. applies & 140. extremity & 159. expenditure \\
\hline 103. rooted & 122. output & 141. implemented & 160. liquids \\
\hline 104. underwent & 123. rebelling & 142. improper & 161. referral \\
\hline 105. comprised & 124. separating & 143. interfere & 162. segment \\
\hline 106. fainting & 125. bleed & 144. standardization & 163. clustering \\
\hline 107. grouping & 126. excessively & 145. encountered & 164. connectivity \\
\hline 108. gentlemen & 127. resistant & 146. gathers & 165. essentials \\
\hline 109. institutional & 128. absorption & 147. localized & 166. rootless \\
\hline 110. interact & 129. colds & 148. reproduction & 167. yielded \\
\hline 111. anticancer & 130. demonstrates & 149. resides & 168. emphasised \\
\hline 112. barriers & 131. exception & 150. sampling & 169. institutes \\
\hline 113. brightens & 132. restricted & 151. uncontrolled & 170. interference \\
\hline 114. fibers & 133. grouped & 152. worsening & 171. normalized \\
\hline 115. immature & 134. mindfulness & 153. clarify & 172. plots \\
\hline 116. injuring & 135. outward & 154. correction & 173. powered \\
\hline 117. maturation & 136. smelling & 155. dislike & \\
\hline 118. references & 137. associations & 156. drainage & \\
\hline 119. salty & 138. ceaseless & 157. piglet & \\
\hline
\end{tabular}




\section{Appendix 9. Sub-list 3 of the TCM technical word list at Level 2}

\begin{tabular}{|c|c|c|c|}
\hline 1. ultrasound & 26. reperfusion & 51. rotundi & 76. potent \\
\hline 2. vein & 27. expectoration & 52. slimy & 77. salvia \\
\hline 3. vitality & 28. itchy & 53. validated & 78. somnolence \\
\hline 4. zedoary & 29. nodules & 54. blazing & 79. albus \\
\hline 5. borborygmus(i) & 30. pertain(s/ing) & 55. cortical & 80. ethanol \\
\hline 6. grandiflori & 31. respiration & 56. cystitis & 81. follicle(s) \\
\hline 7. indirectly & 32. sallow & 57. dispel(s) & 82. phellodendron \\
\hline 8. lateralis & 33. temporal & 58. ephedra & 83. pinellia \\
\hline 9. linear & 34. urticaria & 59. fossa & 84. prostatic \\
\hline 10. cervix & 35. alpha & 60. frutescentis & 85. rib \\
\hline 11. formalin & 36. Bax & 61. macrophages & 86. scraping \\
\hline 12. ginsenoside & 37. conjunction & 62. outpatient & 87. bupleurum \\
\hline 13. polygonati & 38. dyspnea & 63. panacis & 88. dioscorea \\
\hline 14. rhodiola & 39. erection & 64. vitamin & 89. hypogastrium \\
\hline 15. cones & 40. etiology & 65. diastolic & 90. rubrus \\
\hline 16. dipsaci & 41. graminei & 66. guan & 91. stagnate(s) \\
\hline 17. kinase & 42. malleolus & 67. hypothalamus & 92. aggravate \\
\hline 18. orientalis & 43. pathophysiology & 68. rectum & 93. anemarrhena \\
\hline 19. perillae & 44. spatholobi & 69. appendix & 94. clots \\
\hline 20. refined & 45. congenital & 70. calcium & 95. downregulated \\
\hline 21. sigma & 46. corydalis & 71. distress & 96. gentiana \\
\hline 22. cuscutae & 47. dilution & 72. exteriorly & 97. optimal \\
\hline 23. ejaculation & 48. gland(s) & 73. inducing & 98. alternating \\
\hline 24. hiccup(s) & 49. holistic & 74. lacrimation & 99. disinhibit \\
\hline 25. intellect & 50. retinopathy & 75. lucidi & 100. hypochondriac \\
\hline
\end{tabular}


Continued

\begin{tabular}{|l|l|l|l|}
\hline 101. lethargy & 126. bland & 151. aconite & 176. murky \\
\hline 102. magnolia & 127. bronchitis & 152. aphasia & 177. proteinuria \\
\hline 103. polydipsia & 128. flaccidity & 153. codonopsitis & 178. susceptible \\
\hline 104. amelioration & 129. flaring & 154. colitis & 179. visceral \\
\hline 105. endocrine & 130. goitre & 155. fracture & 180. dependence \\
\hline 106. fallopian & 131. malignant & 156. incubation & 181. dissolved \\
\hline 107. forsythiae & 132. resentment & 157. pectoris & 182. draconis \\
\hline 108. metabolites & 133. umbilical & 158. rectify & 183. enlarged \\
\hline 109. penetration & 134. vagina & 159. spinous & 184. ingestion \\
\hline 110. seizures & 135. airway & 160. stimuli & 185. nodes \\
\hline 111. static & 136. cramps & 161. acoustic & 186. relapse \\
\hline 112. zizyphi & 137. dysentery & 162. autophagy & 187. sepsis \\
\hline 113. adjunctive & 138. ion & 163. chrysanthemi & 188. benign \\
\hline 114. algorithm & 139. licorice & 164. dysenteric & 189. diuresis \\
\hline 115. apoptotic & 140. mucosa & 165. expulsion & 190. dopamine \\
\hline 116. bowels & 141. neural & 166.interrogation & 191. rectal \\
\hline 117. cannabis & 142. rubra & 167. lonicerae & 192. traumatic \\
\hline 118. conquitae & 143.transplantation & 168. regimen & 193. urethra \\
\hline 119. fetal & 144. vertex & 169. turmoil & 194. artemisiae \\
\hline 120. flush & 145. alternation & 170. basel & 195. cohort \\
\hline 121. junction & 146.cerebrovascular & 171. cyathulae & 196. follicular \\
\hline 122. menopause & 147. fortify(ies) & 172. differential & 197. funnel \\
\hline 123.pneumothorax & 148.proinflammatory & 173. ephedrae & 198. hyperplasia \\
\hline 124. sclerosis & 149. stifling & 174. gastritis & 199. myopia \\
\hline 125. thoracic & 150. validation & 175. identical & 200. phosphate \\
\hline
\end{tabular}


Continued

\begin{tabular}{|c|c|c|c|}
\hline 201. sui & 226. pancreatitis & 251. aetiological & 276. ovarian \\
\hline 202. ulceration & 227. postpartum & 252. anemia & 277. Pon 2 \\
\hline 203. viability & 228. sandalwood & 253. anesthetized & 278. sacrum \\
\hline 204. absent & 229. artemisia & 254. bilaterally & 279. subcutaneous \\
\hline 205. actin & 230. ascites & 255. colorectal & 280. throbbing \\
\hline 206.neuroprotective & 231. bambusae & 256. flabby & 281. tuberculosis \\
\hline 207. peritoneal & 232. centrifuged & 257. indirect & 282. clonic \\
\hline 208. saussureae & 233. cocaine & 258. morifolii & 283. dysmenorrhoea \\
\hline 209. skeletal & 234. congealed & 259. stubborn & 284. eligibility \\
\hline 210. subjected & 235. fritillariae & 260. treatise & 285. ginkgo \\
\hline 211. acrid & 236. guinea & 261. autoimmune & 286. inhalation \\
\hline 212. cholinergic & 237. insulting & 262. elucidated & 287. lignum \\
\hline 213. eruptions & 238. mitochondria & 263. glehniae & 288. nelumbinis \\
\hline 214. menses & 239. p38 & 264. hemoglobin & 289. opisthotonos \\
\hline 215. perineum & 240. testicles & 265. histamine & 290. overweight \\
\hline 216. polyuria & 241. augument(ed) & 266. noninvasive & 291. sterile \\
\hline 217. rigorous & 242. denotes & 267. occiput & 292. viscera \\
\hline 218. sequelae & 243. duct & 268. palpable & 293. adjuvant \\
\hline 219. thyriod & 244. gentianae & 269. prenatal & 294. affinity \\
\hline 220. compliance & 245. ledebouriellae & 270. quantified & 295. ancestral \\
\hline 221. contrary & 246. logistic & 271. ascent & 296.chromatography \\
\hline 222. crataegi & 247. menthae & 272. astringe & 297. dysmenorrhea \\
\hline 223. dermatomes & 248. phosphorylation & 273. corpus & 298. elicited \\
\hline 224. frankincense & 249. prevalent & 274. distilled & 299. interleukin \\
\hline 225. malar & 250. suspensae & 275. impair(s) & 300. location \\
\hline
\end{tabular}


Continued

\begin{tabular}{|c|c|c|c|}
\hline 301. lobe & 326. obstructive & 351.pseudostellariae & 376. podocyte \\
\hline 302. metformin & 327. oxalate & 352. sinusitis & 377. polarity \\
\hline 303. mucous & 328. regent(s) & 353. anesthesia & 378. pubic \\
\hline 304. otitis & 329. vexation & 354. cuscatae & 379. utilization \\
\hline 305. polymerase & 330. follicle(s) & 355. exert & 380. X-ray \\
\hline 306. postnatal & 331. harmonious & 356. interstitial & 381. asari \\
\hline 307. anatomy & 332. morbidity & 357. popliteal & 382. clonic \\
\hline 308. carbohydrates & 333. morphine & 358. purulent & 383. comparative \\
\hline 309. cimicifugae & 334. resonance & 359. serotonin & 384. erectile \\
\hline 310. electrophoresis & 335. retrieved & 360. suppression & 385. glycyrrhiza \\
\hline 311. extracellular & 336. sacral & 361. susceptibility & 386. microscope \\
\hline 312. genitalia & 337. uncariae & 362. testudinis & 387. muzziness \\
\hline 313. histone & 338. verified & 363. apparatus & 388. nucleus \\
\hline 314. littoralis & 339. adherence & 364. asthmatic & 389. pacify \\
\hline 315. measles & 340. attenuated & 365. constriction & 390. usage \\
\hline 316. nitrogen & 341. cartilage & 366. cumulative & 391. antiarrhythmic \\
\hline 317. nocturia & 342. crease & 367. infract & 392. antibiotic \\
\hline 318. psoriatic & 343. degeneration & 368. inherent & 393. Ca1 \\
\hline 319. simulated & 344. ectopic & 369. ligustri & 394. cytosolic \\
\hline 320. suppressed(ed) & 345. harmonized & 370. lipoprotein & 395. electro \\
\hline 321. afferent & 346. impair(s) & 371. metastasis & 396. ligand \\
\hline 322. biomedicine & 347. infantile & 372. microarray & 397. massa \\
\hline 323. fibroblasts & 348. multiflorum & 373. morindae & 398. plantago \\
\hline 324. intraperitoneal & 349. pediatrics & 374. multivariate & 399. pollen \\
\hline 325. modulation & 350. prominence & 375.pharmacotherapy & 400. purging \\
\hline
\end{tabular}


Continued

\begin{tabular}{|l|l|l|l|}
\hline 401. soles & 408. sedation & 415. consolidation & 422. parching \\
\hline 402. soothe & 409. spatial & 416. gradient & 423. sesloidis \\
\hline 403. transient & 410. twitching & 417.immunoistochemistry & 424. sphere \\
\hline 404. creatinine & 411. amino & 418. intercourse & 425. stuffiness \\
\hline 405. dorsal & 412. appendicitis & 419. kaempferol & 426.supraclavicular \\
\hline 406. macrocephala & 413. bloating & 420. notopterygii & \\
\hline 407. oxide & 414. bouts & 421. nutrients & \\
\hline
\end{tabular}




\section{Appendix 10. Sub-list 3 of the TCM technical word list at Level 3}

\begin{tabular}{|l|l|l|l|}
\hline 1. fuling & 22. chengshan & 43. touwei & 64. lidui \\
\hline 2. shanghuo & 23. ciliao & 44. weiyang & 65. qiwei \\
\hline 3. zhangmen & 24. laogong & 45. yanhusuo & 66. renying \\
\hline 4. zhongfu & 25. qiuxu & 46. yinbai & 67. shenque \\
\hline 5. liang & 26. shousanli & 47. tianzhu & 68. shenzhu \\
\hline 6. mang & 27. yingu & 48. xiaxi & 69. jinmen \\
\hline 7. fengmen & 28. dadun & 49. yintang & 70. shangwan \\
\hline 8. chuanxiong & 29. jianjing & 50. changqiang & 71. tui \\
\hline 9. jiexi & 30. yangxi & 51. tou & 72. yingxiang \\
\hline 10. dachangshu & 31. guo & 52. siman & 73. daheng \\
\hline 11. yongquan & 32. huai & 53. tianjing & 74. dashu \\
\hline 12. shangxing & 33. shaoyang & 54. miao & 75. lao \\
\hline 13. wangu & 34. tiantu & 55. shimen & 76. yangfu \\
\hline 14. dao & 35. guizhi & 56. zhengs & 77. shaoze \\
\hline 15. guasha & 36. jingming & 57. guanchong & 78. ao \\
\hline 16. ligou & 37. bufei & 58. shangyang & 79. tuina \\
\hline 17. qichong & 38. xiajuxu & 59. shaoshang & 80. ahshi \\
\hline 18. xuanzhong & 39. yanggu & 60. feiyang & 81. jueyinshu \\
\hline 19. zhongzhu & 40. jianshi & 61. sanjian & 82. shaohai \\
\hline 20. shangqiu & 41. zuo & 62. fengshi & 83. yamen \\
\hline 21. juque & 42. dazhong & 63. huiyin & \\
\hline
\end{tabular}




\section{Appendix 11. TCM Technical lexical bundles at Category 1}

(arranged by their number of occurrences in the TCM Corpora)

\begin{tabular}{|c|c|c|}
\hline 1. (zhi) gan cao & 31. shao yao & 61. shi chang pu \\
\hline 2. fu ling & 32. (chuan/huai) niu xi & 62. che qian zi \\
\hline 3. dang gui & 33. mu dan pi & 63. ge gen \\
\hline 4. bai zhu & 34. hou po & 64. di huang wan \\
\hline 5. huang qi & 35. yi yi ren & 65. fang feng \\
\hline 6. sheng di (huang) & 36. xuan shen & 66. ju hua \\
\hline 7. bai shao (yao) & 37. zhong yi & 67. du zhong \\
\hline 8. ban xia & 38. gou qi zi & 68. sha shen \\
\hline 9. huang qin & 39. cang zhu & 69. yuan zhi \\
\hline 10. ren shen & 40. mu xiang & 70. lian zi \\
\hline 11. chen pi & 41. tao ren & 71. bei mu \\
\hline 12. (shan/wu) zhu yu & 42. shi gao & 72. huo xiang \\
\hline 13. huang lian & 43. xin ren & 73. jin yin hua \\
\hline 14. mai men dong & 44. da zao & 74. yuan qi \\
\hline 15. chai hu & 45. ma huang & 75. gou teng \\
\hline 16. dan shen & 46. hong hua & 76. sha ren \\
\hline 17. shu di (huang) & 47. suan zao ren & 77. e jiao \\
\hline 18. gui zhi & 48. rou gui & 78. zheng qi \\
\hline 19. shan yao & 49. gan jiang & 79. yan hu suo \\
\hline 20. (lai/zhi) fu zi & 50. tu si zi & 80. shan zha \\
\hline 21. chuang xiong & 51. jie geng & 81. hua shi \\
\hline 22. da huang & 52. mu li & 82. yang ming \\
\hline 23. wu wei zi & 53. tian hua fen & 83. bo he \\
\hline 24. ze xie & 54. xiang fu & 84. xi xin \\
\hline 25. (shan) zhi zi & 55. chang pu & 85. mu tong \\
\hline 26. (the) yang qi & 56. (the) san jiao & 86. zhu ru \\
\hline 27. dang shen & 57. gua lou & 87. sheng ma \\
\hline 28. sheng jiang & 58. huang bai & 88. Nei Jing \\
\hline 29. chi shao (yao) & 59. lian qiao & 89. Shang Han Lun \\
\hline 30. zhu mu & 60. yu jin & 90. zhu ling \\
\hline
\end{tabular}


Continued

\begin{tabular}{|l|l|l|}
\hline 91. tian ma & 114. mang xiao & 137. zhe bei mu \\
\hline 92. xu duan & 115. pu gong ying & 138. (bai) dou kou \\
\hline 93. shen qi & 116. lu jiao jiao & 139. ji ju \\
\hline 94. yin chen hao & 117. rou cong rong & 140. yi mu cao \\
\hline 95. qiang huo & 118. tian men dong & 141. ba ji tian \\
\hline 96. tai zi shen & 119. bu gu zhi & 142. mu gua \\
\hline 97. long gu & 120. di long & 143. ru xiang \\
\hline 98. shen qu & 121. he shou wu & 144. cheng qi tang \\
\hline 99. Fang Ji & 122. zi su (zi/ye) & 145. du huo \\
\hline 100. sang bai pi & 123. long dan cao & 146. mo yao \\
\hline 101. zhu ye & 124. wei qi & 147. sang ye \\
\hline 102. bai zhi & 125. jing jie & 148. bian dou \\
\hline 103. ji xue teng & 126. liu wei di huang wan & 149. (bai) mao gen \\
\hline 104. san qi & 127. sang ji sheng & 150. chong mai \\
\hline 105. bu zhong yi qi tang & 128. di gu pi & 151. su ye \\
\hline 106. Jin Gui Yao Lue & 129. tai yang & 152.xiao ji \\
\hline 107. dan nan xing & 130. wu yao & 153. liu jun zi tang \\
\hline 108. huang bo & 131. xiao yao san & 154. zhen zhu (mu) \\
\hline 109. nv zhen zi & 132. pu huang & 156. ren mai \\
\hline 110. shen qi wan & 133. shao yang & 159. xia ku cao \\
\hline 111. chen xiang & 134. mai ya yang huo \\
\hline 112. chuan lian zi & 135. si wu tang & \\
\hline 113. shi hu & 136. e zhu & 15ing \\
\hline
\end{tabular}




\section{Appendix 12. TCM Technical lexical bundles at Category 2}

(arranged by their number of occurrences in the TCM Corpora)

1. (the) yin deficiency

2. (spleen and/tonify/tonifies/deficiency of/the/warm) kidney yang

3.(stagnation of/flow of/ moves/the/stagnant/rebellious/free flow of) liver qi

4. (the) kidney yin

5. (descending of/the/tonify/tonifies) lung qi (deficiency)

6. (heart/stomach/kidney) qi deficiency

7. (liver) qi stagnation

8. (spleen/spleen and kidney/heart) yang deficiency

9. (the/tonify/tonifies/ascending of/deficiency of/the ascending of/deficient) spleen qi

10. (subdues/subdue rising of) liver yang (rising)

11. (descending of/the descending of/rebellious) stomach qi

12. (the/nutritive and) defensive qi (level)

13. (the/in the/from the/qi in the/dampness from the/fluids in the) lower burner

14. (nourish) liver yin (deficiency)

15. kidney qi (pill)

16. (the) heart qi

17. (subdues/subdue) rebellious qi

18. (the/in the) middle jiao

19. stagnation of qi (and blood)

20. (to) nourish yin

21. (the) original qi

22. (the/in the/middle and) lower jiao

23. (the) yang channels

24. spleen yang

25. (a/hui) meeting point (of the gallbladder)

26. tonify qi (and blood)

27. (the) nutritive qi (level)

28. (kidney) yin vacuity

29. (the/six) yang organs

30. (the) upright qi 
Continued

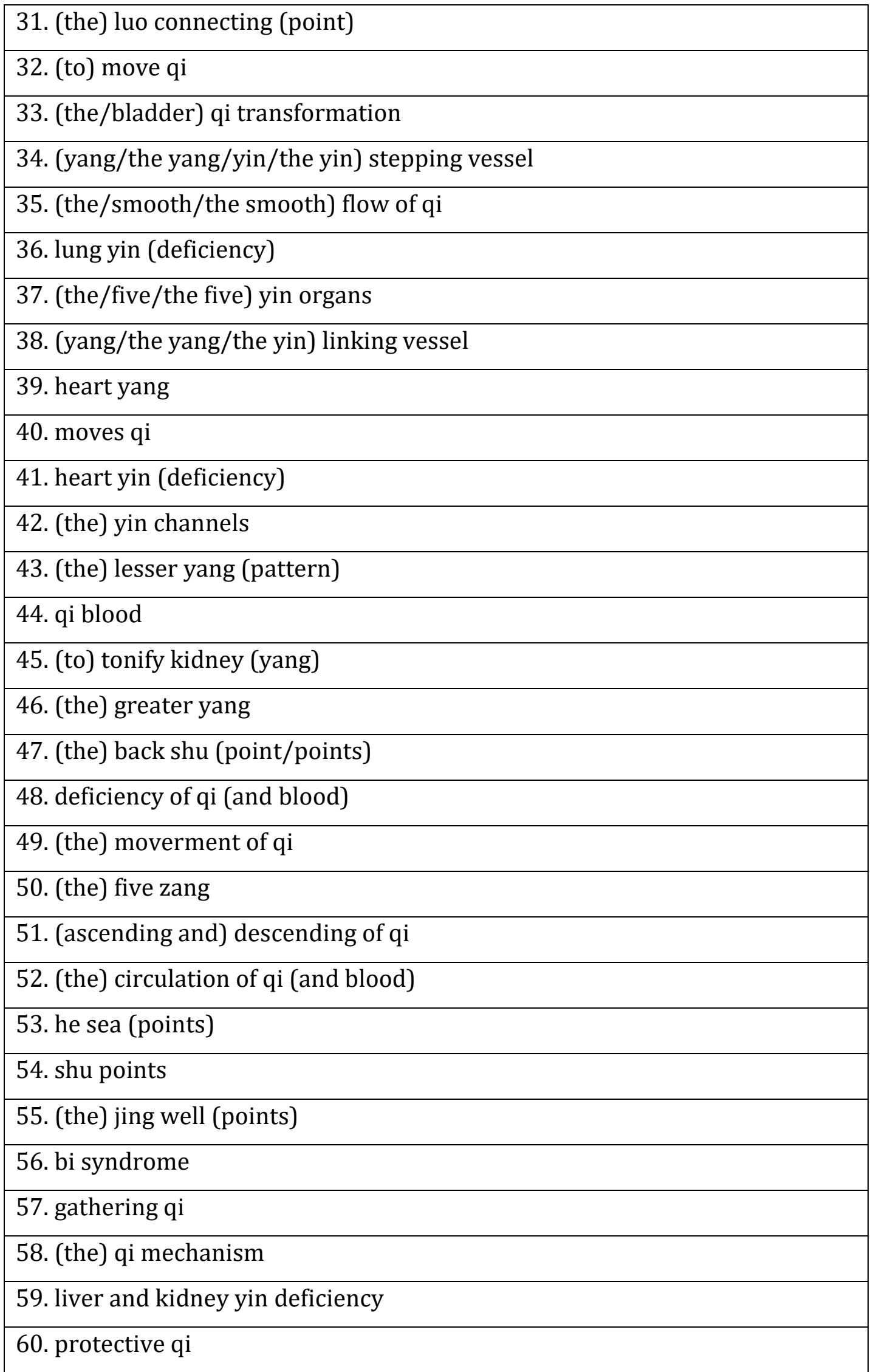


Continued

61. (the) upper jiao

62. deficiency of yang

63. clear yang

64. food qi

65. nourishes yin

66. yang vacuity

67. (post) heaven qi

68. qi flow

69. deficiency of yin

70. the bright yang

71. deficient qi

72. (the) front mu (point)

73. regulates qi

74. ye fluids

75. excess of yang

76. nourishing yin

77. xi cleft (point/points)

78. yin fluids

79. (the yang) motility vessel

80. the qi decoction

81. yang channel

82. regulate $\mathrm{qi}$

83. the body's qi

84. mu point

85. sea of qi

86. stomach yin deficiency

87. yin fire

88. deficiency of kidney yin

89. rectify the qi 


\section{Appendix 13. TCM Technical lexical bundles at Category 3}

1. (traditional) Chinese medicine (practitioners)

2. (resolves/the/resolve) damp heat

3. (qi stagnation and/liver/phlegm and/heart) blood stasis (syndrome)

4. (the/in the/compared with the/a) control group

5. (the) treatment principle (of)

6. (the) clinical manifestations (of)

7. (chest/chronic) painful obstruction

8. in the treatment (of)

9. (the/in the/heat in the) large intestine (channel)

10. (liver/heart/qi and) blood deficiency

11. in Western medicine

12. (lower) abdominal pain

13. (nourish/deficiency of/nourishes) liver blood

14. (exterior/external) pathogenic factors

15. (the/in the) small intestine (channel)

16. clear heat (from)

17. (invasion of/exterior/expels/expel/an invasion of) wind heat

18. (the/a/exterior/an exterior/ residual/a residual/expel) pathogenic factor 19. wind cold (invasion)

20. (yellow/the) tongue coating

21. (a/dark) red tongue (without coating/with yellow coating)

22. (the/in the) penetrating vessel

23. (qi/pain/blood/fullness) in the chest

24. (the) acupuncture points

25. (sclerotium) poriae cocos

26. liver fire

27. (the) treatment principles

28. (a/due to/liver and) kidney deficiency

29. (painful/chronic painful) obstruction syndrome

30. (before and) after treatment 
Continued

31. (the) acupuncture treatment

32. (the/settle the/settles the) ethereal soul

33. (Chinese) herbal medicine

34. (the) governing vessel

35. (fine/floating/a/slippery) rapid pulse

36. (high/diastolic/the) blood pressure

37. (yin deficiency with) empty heat

38. (the) triple burner

39. clears heat (from)

40. supplementing life

41. radix angelicae sinensis

42. (the/in the) treatment group

43. (the) internal organs

44. (fasting) blood glucose

45. significant difference

46. (radix) glycyrrhizae uralensis

47. (in) clinical trails

48. calms the mind

49. (low/lower/chronic low) back pain

50. spleen deficiency

51. herbal therapy

52. adverse events

53. aversion to cold

54. for the treatment (of)

55. in this case

56. heart blood

57. (the) lower back

58. (the) urinary bladder

59. atractylodis macrocephalae (rhizoma)

60. (the/in the/qi in the) lower abdomen 
Continued

61. (the/blood and) body fluids

62. (the) side effects

63. shortness of breath

64. (in) clinical practice

65. (the) kidney essence (deficiency)

66. symptoms and signs

67. (the) blood vessels

68. main indication

69. blurred vision

70. resolve dampness

71. (heat) in the stomach

72. poor appetite

73. radix glycyrrhizae uralensis

74. (all with) reinforcing method

75. (to) calming the mind

76. (health related/the) quality of life

77. (pericarpium) citri reticulatae

78. stasis of blood

79. (a) bitter taste (in the mouth)

80. significant differences

81. herbal treatment

82. rhizoma atractylodis macrocephalae

83. (small) sample size

84. statistical analysis

85. (sticky/tongue with) yellow coating

86. loose stools

87. cinnamomi cassiae

88. (the) corporeal soul

89. (a) feeling of heaviness

90. (with/needled with) reducing method 
Continued

91. (the/the blood) connecting channels

92. resolves dampness

93. thin mucus

94. wind stroke

95. (the/in the) liver channel

96. herbal formula

97. (to) expel wind

98. rehanniae glutinosae (conquitae)

99. (invasion of/expels) exterior wind

100. case history

101. abdominal distension (and pain)

102. (the) bladder channel

103. (lateral/the lateral) costal region

104. paeoniae lactiflorae

105. phlegm heat

106. expels wind

107. syndrome treatment (principle)

108. (to) resolve phlegm

109. difficult urination

110. (a) pale tongue

111. ophiopohonis japonici

112. (radix) salviae miltiorrhizae

113. radix astragali membranacei

114. (the) directing vessel

115. dry throat

116. (excessive) sexual activity

117. connecting channel

118. (heat/phlegm) in the lungs

119. tuber ophiopogonis (japonici)

120. (a/important) local point 
Continued

121. (clears) stomach heat

122. dioscoreae oppositae

123. (the) main symptoms

124. blood stagnation

125. controlled trails

126. epigastric pain

127. abdominal distention

128. (the) extraordinary vessels

129. invigorate blood

130. chest pain

131. liver wind

132. mental restlessness

133. radix rehmanniae glutinosae (conquitae)

134. related conditions

135. invasion of wind

136. urinary syndrome

137. Chinese herbs

138. (scanty) dark urine

139. health care

140. core formula

141. distil points

142. (central/the central) nervous system

143. stop bleeding

144. body weight

145. (rhizoma) pinelliae ternatae

146. poor memory

147. (the) five element

148. (heat/damp heat) in the liver

149. before treatment

150. (clears/clear) lung heat 
Continued

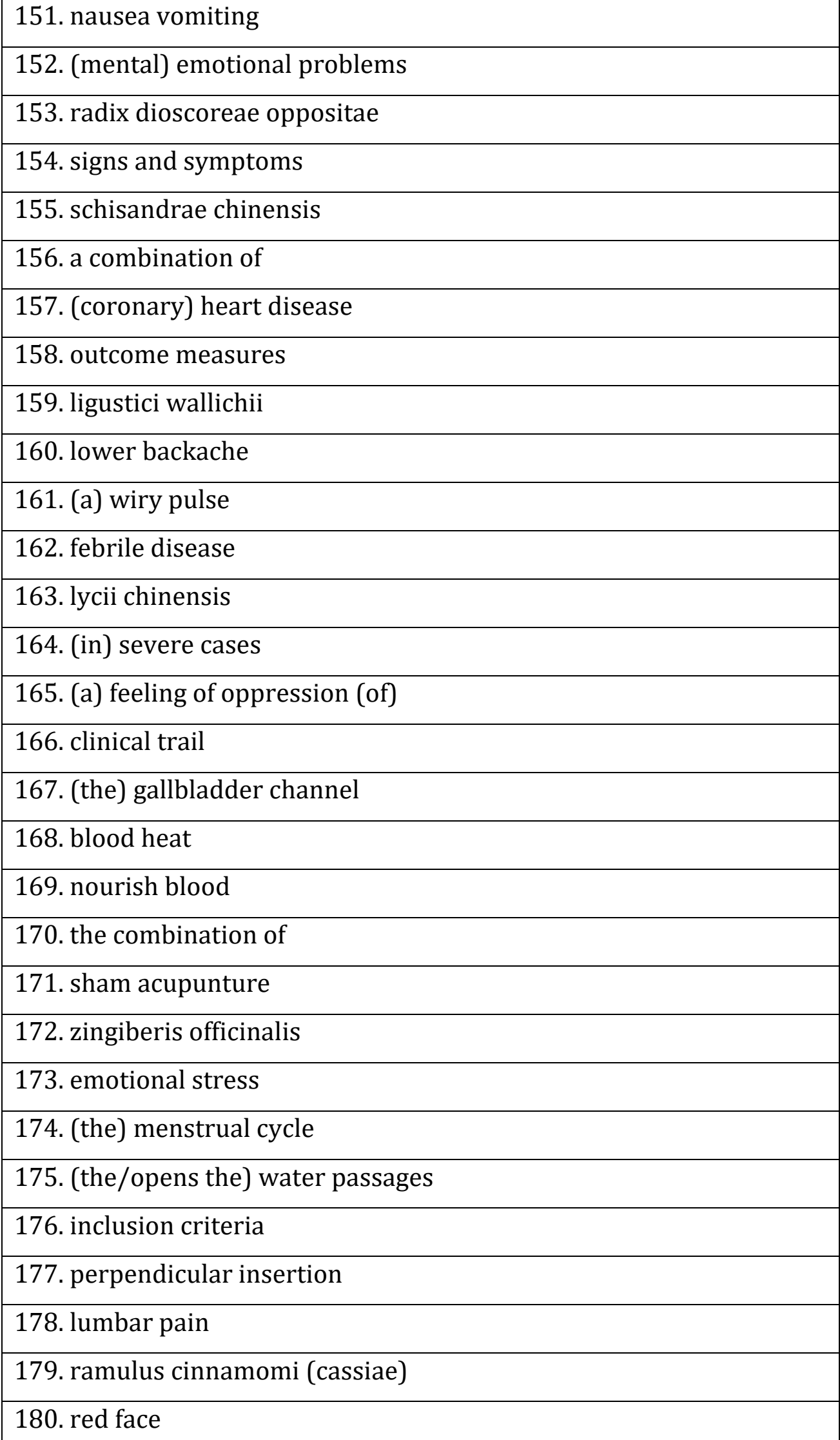


Continued

181. sinew channel

182. corni officinalis

183. the four limbs

184. dizziness tinnitus

185. cold limbs

186. (the) stomach channel

187. in vitro

188. radix ligustici wallichii

189. scutellariae baicalensis

190. fatigue syndrome

191. summer heat

192. interior wind

193. in excess (patterns)

194. fructus schisandrae chinensis

195. adverse effects

196. external wind

197. (the/in the) middle burner

198. blood flow

199. in the head

200. chinese medicines

201. chronic fatigue

202. radix bupleuri

203. deficiency syndrome

204. phlegm damp

205. (the) five elements

206. general prescription

207. to clear heat (from)

208. (the) clinical symptoms

209. (a) slippery pulse

210. heart pain 
Continued

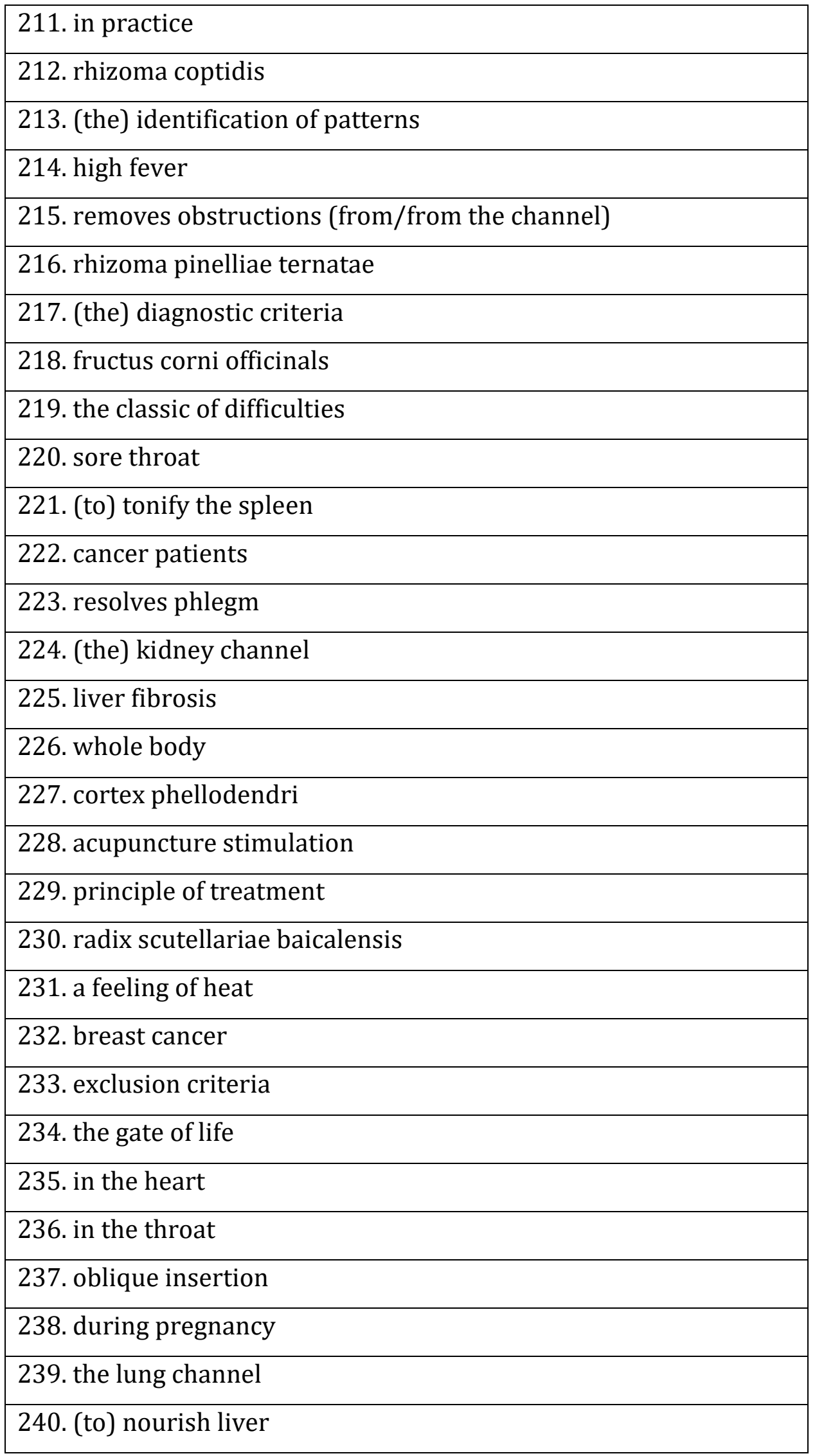


Continued

\begin{tabular}{|l|}
\hline 241. (sticky) yellow tongue (coating) \\
\hline 242. alleviates pain \\
\hline 243. blood sugar (levels) \\
\hline 244. fructus lycii chinensis \\
\hline 245. retention of urine \\
\hline 246. (a/as a) distil point \\
\hline 247. (the) effects of acupuncture \\
\hline 248. (the) excessive consumption of \\
\hline 249. (the) smooth flow of \\
\hline 250. syndrome differentiation \\
\hline 251. the lumbar region \\
\hline 252. transform phlegm \\
\hline 253. fullness of the chest \\
\hline 254. pattern differentiation \\
\hline 255. cold damp \\
\hline 256. (yellow) greasy tongue (coating) \\
\hline 257. invigorates blood \\
\hline 258. pulse treatment \\
\hline 259. radix glycyrrhizae uralensis praeparata \\
\hline 260. retention of food \\
\hline 261. (Chinese) herbal medicines \\
\hline 262. lack of strength \\
\hline 263. clinical note \\
\hline 264. headache dizziness \\
\hline 265. radix trichosanthis \\
\hline 266. divergent channel \\
\hline 267. stop pain \\
\hline 268. control groups \\
\hline 269. \\
\hline
\end{tabular}


Continued

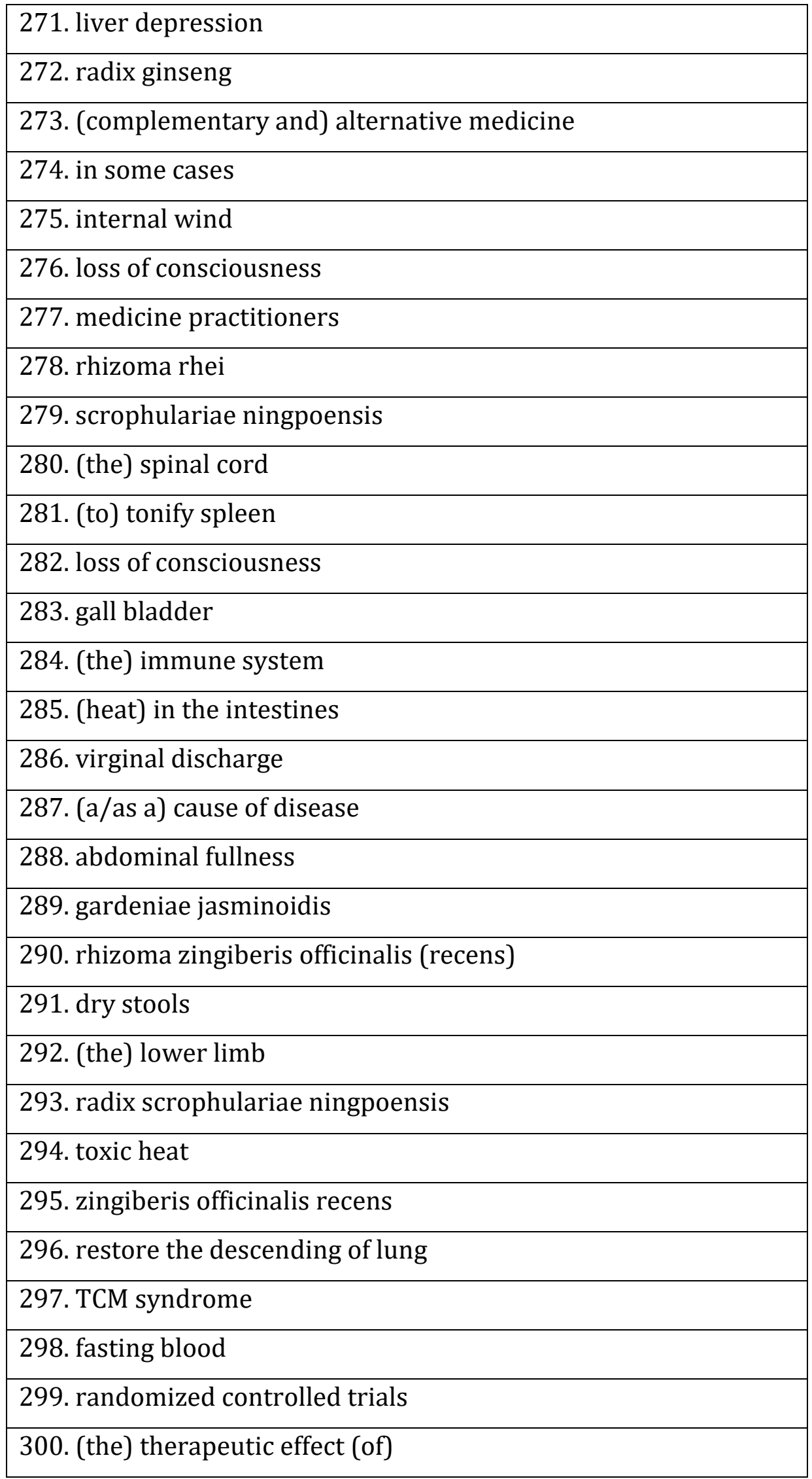


Continued

\begin{tabular}{|l|}
\hline 301. wind damp \\
\hline 302. blood circulation \\
\hline 303. diabetes mellitus \\
\hline 304. in the abdomen \\
\hline 305.night sweats \\
\hline 306. abdominal masses \\
\hline 307. magnoliae officinalis \\
\hline 308. (thin) white coating \\
\hline 309. (radix) achyranthis bidentatae \\
\hline 310. t test \\
\hline 311. TCM treatment \\
\hline 312. undigested food (in the stool) \\
\hline 313. frequent urination \\
\hline 314. (damp heat) in the bladder \\
\hline 315. rheumatoid arthritis \\
\hline 316. carthami tinctorii \\
\hline 317. (blood) in the uterus \\
\hline 318. obstructions from the channel \\
\hline 319. (thin) white tongue (coating) \\
\hline 320. controlled trail \\
\hline 321. (the) early stage \\
\hline 322. human body \\
\hline 323. right side \\
\hline 324. stiff neck \\
\hline 325. (space) between skin and muscles \\
\hline 326. the effect of acupuncture \\
\hline 327. protein expression \\
\hline
\end{tabular}


Continued

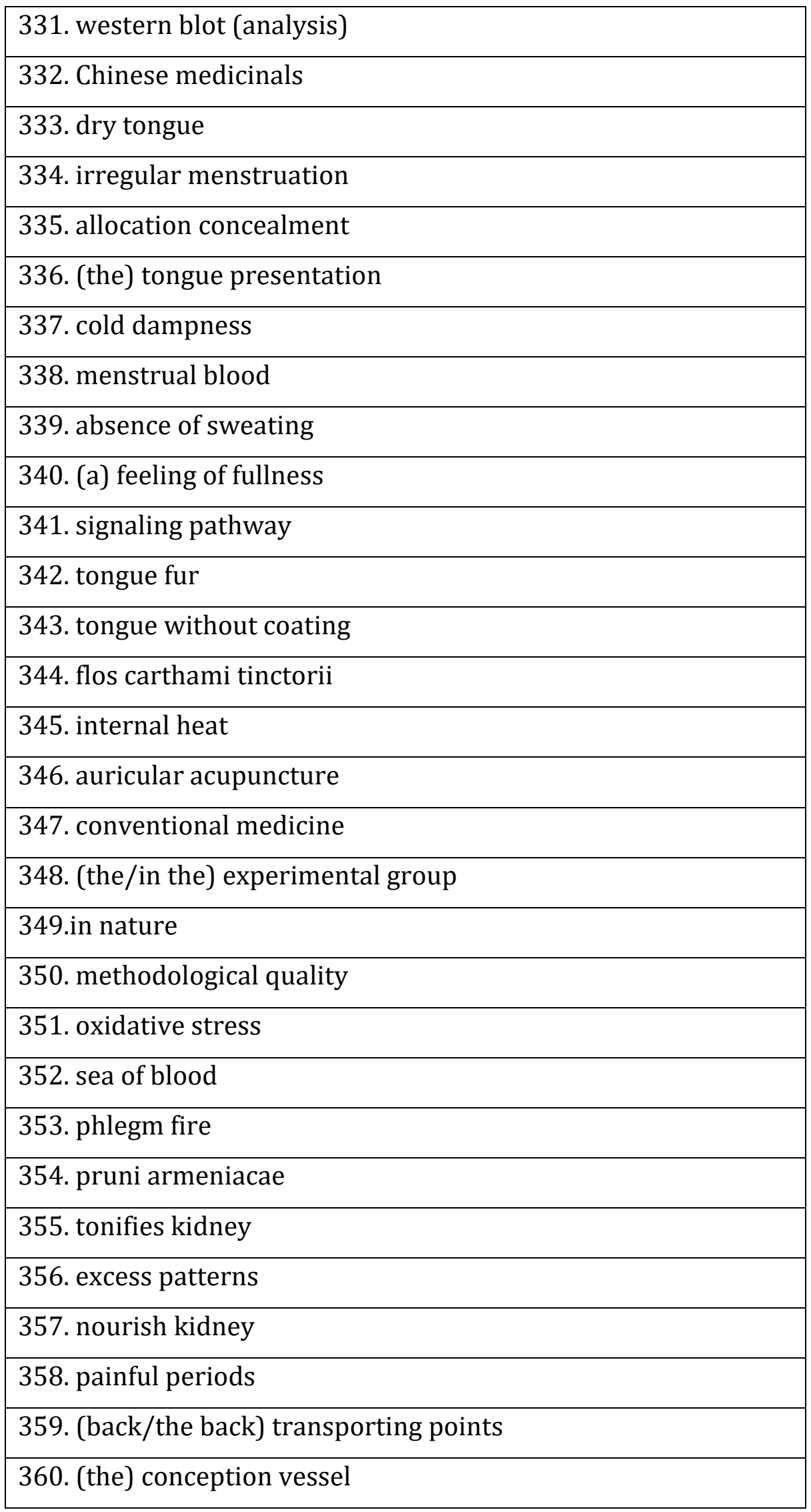


Continued

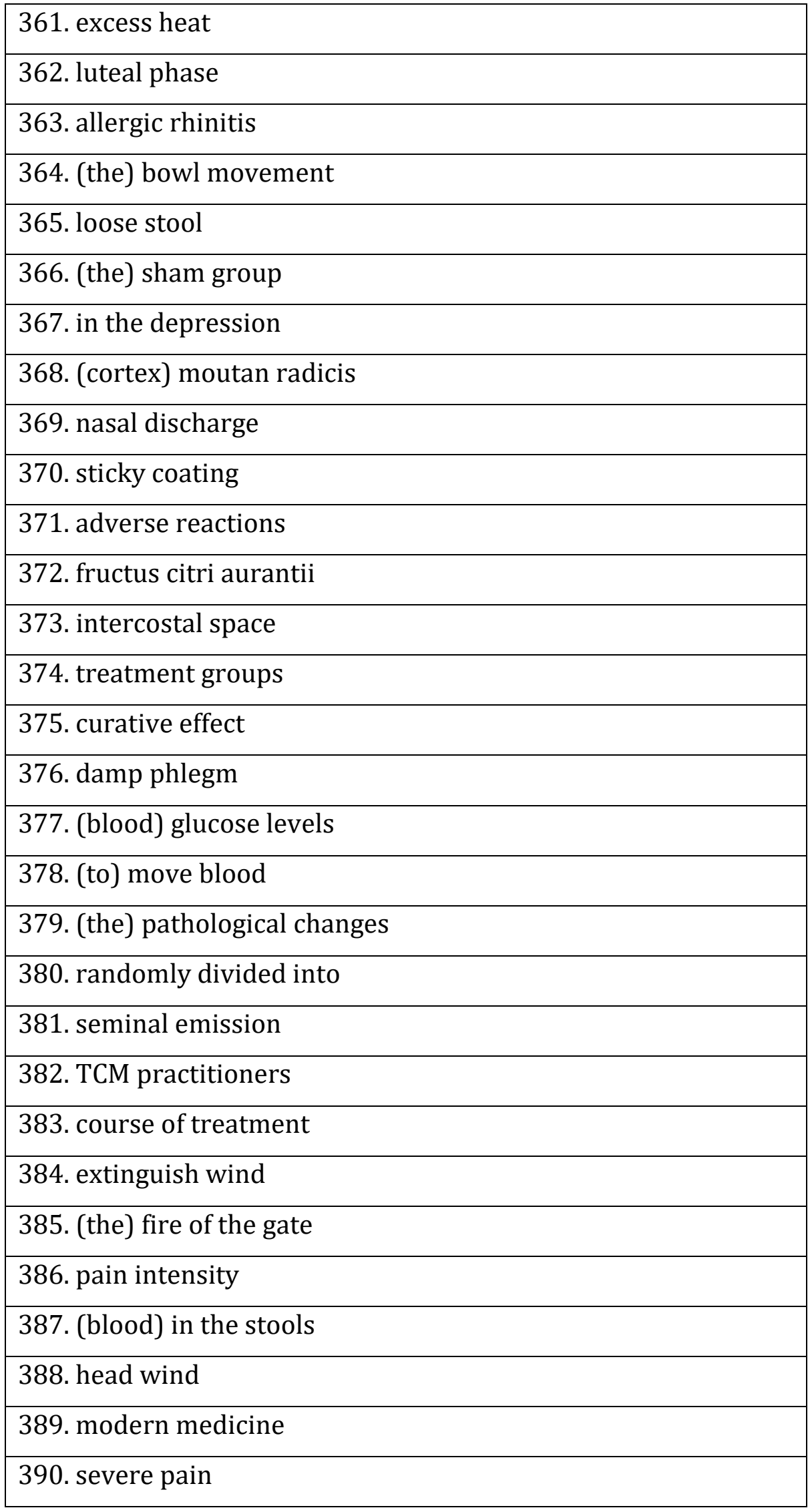


Continued

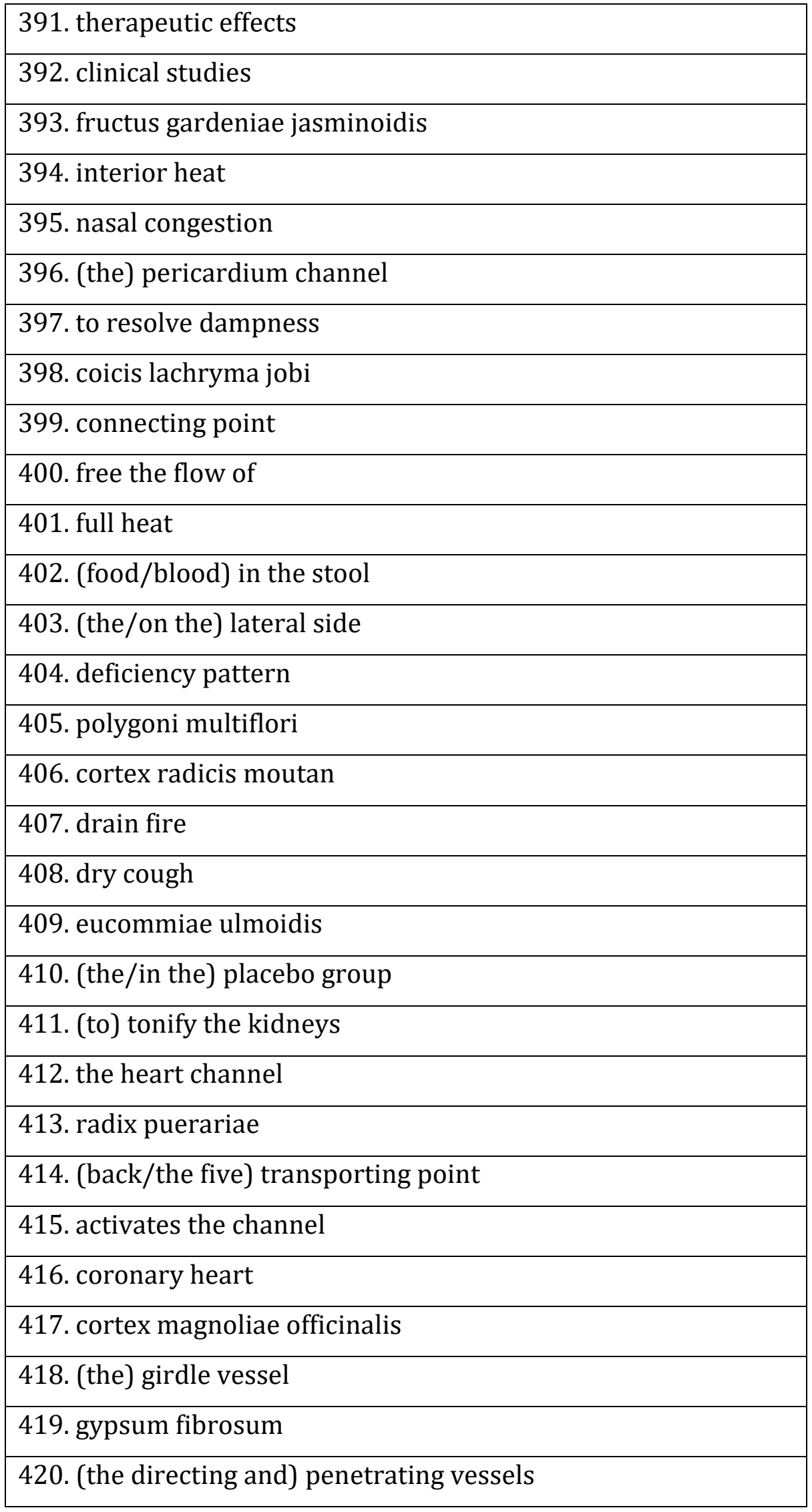


Continued

\begin{tabular}{|l|}
\hline 421. spontaneous sweating \\
\hline 422. cornu cervi \\
\hline 423. rehmannia pill \\
\hline 424. atrophy disorder \\
\hline 425. platycodi grandiflori \\
\hline 426.causes of disease \\
\hline 427. (the most) common cause of \\
\hline 428. pale complexion \\
\hline 429. cortex cinnamomi (cassiae) \\
\hline 430. in the model group \\
\hline 431. manic behaviour \\
\hline 432. nourishes blood \\
\hline 433. essential oil \\
\hline 434. (the) main channels \\
\hline 435. (the) main points \\
\hline 436. chronic pain \\
\hline 437. clinical experience \\
\hline 438. latent heat \\
\hline 439. semen coicis lachryma jobi \\
\hline 440. (painful) urinary dysfunction \\
\hline 441. cell proliferation \\
\hline 442. cool blood \\
\hline 443. febrile diseases \\
\hline 444. heart rate \\
\hline 445. nourishes liver \\
\hline 446. spleen vacuity \\
\hline 447. (in) the acupuncture group \\
\hline 448. the sea of blood \\
\hline 449. urinary retention \\
\hline 450. ang of cold \\
\hline
\end{tabular}


Continued

\begin{tabular}{|l|}
\hline 451. acori graminei \\
\hline 452. at room temperature \\
\hline 453. chronic conditions \\
\hline 454. gene expression \\
\hline 455. heat syndrome \\
\hline 456. neck pain \\
\hline 457. pain relief \\
\hline 458. radix et rhizoma rhei \\
\hline 459. red eyes \\
\hline 460. throat painful obstruction \\
\hline 461. counter flow \\
\hline 462. (floating) empty pulse \\
\hline 463. focal distention \\
\hline 464. heart failure \\
\hline 465. hepatitis B \\
\hline 466. rhizoma polygonati \\
\hline 467. semen pruni armeniacae \\
\hline 468. (rhizoma) cyperi rotundi \\
\hline 469. deficiency of blood \\
\hline 470. extra point \\
\hline 471. hypochondrial pain \\
\hline 472. (fructus) perillae frutescentis \\
\hline 473. redness swelling \\
\hline 474. acupuncture treatments \\
\hline 475. bone marrow \\
\hline 476. bowel movements \\
\hline 477. cortex eucommiae ulmoidis \\
\hline 478. patent remedy \\
\hline 479. restores the descending of lung \\
\hline 480.reae \\
\hline 460 \\
\hline
\end{tabular}


Continued

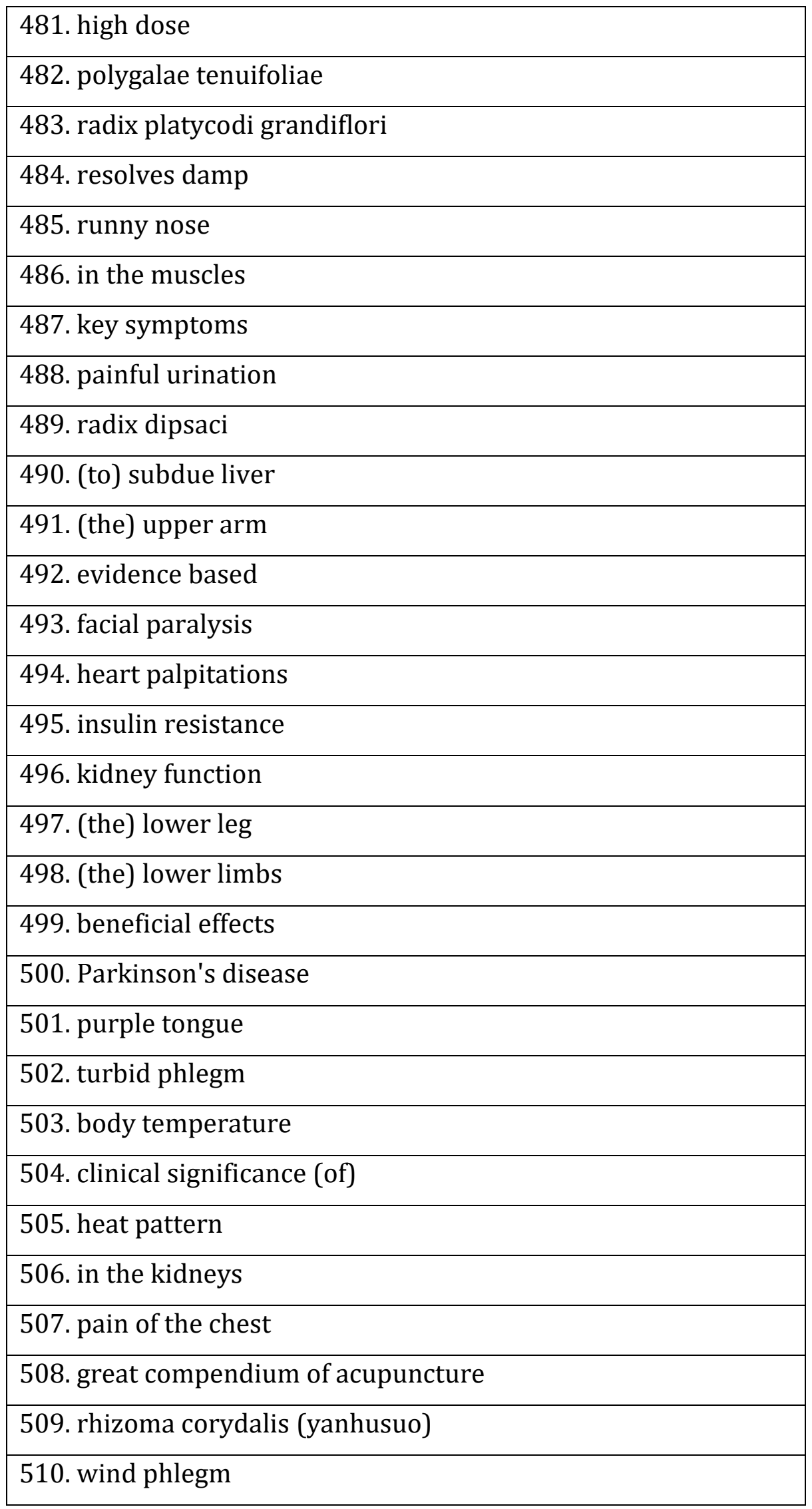


Continued

\begin{tabular}{|l|}
\hline 511. skin diseases \\
\hline 512. spontaneous perspiration \\
\hline 513. throughout the body \\
\hline 514. urinary tract \\
\hline 515. acute cases \\
\hline 516. lateral to the midline \\
\hline 517. oral administration \\
\hline 518. physical activity \\
\hline 519. semen ziziphi spinosae \\
\hline 520. sour regurgitation \\
\hline 521. under the skin \\
\hline 522. eliminate stasis \\
\hline 523. relieve toxicity \\
\hline 524. sleep quality \\
\hline 525. stomach fire \\
\hline 526. angina pectoris \\
\hline 527. atrophy syndrome \\
\hline 528. food stagnation \\
\hline 529. pain of the shoulder \\
\hline 530. (the) right kidney \\
\hline 531. slow pulse \\
\hline 532. (the) spleen channel \\
\hline 533. stops pain \\
\hline 534. the lateral side of \\
\hline 535. warm diseases \\
\hline 536. kidney pill \\
\hline 537. national health \\
\hline 538. pathogenic wind \\
\hline 539. radix polygalae tenuifoliae \\
\hline 540. disease mechanisms \\
\hline 52 \\
\hline
\end{tabular}


Continued

\begin{tabular}{|l|}
\hline 541. efficacy and safety of \\
\hline 542. eliminate dampness \\
\hline 543. phlegm dampness \\
\hline 544. semen cuscutae \\
\hline 545. weak voice \\
\hline 546. deficient cold \\
\hline 547. emotional strain \\
\hline 548. liver injury \\
\hline 549. stops bleeding \\
\hline 550. sudden turmoil (disorder) \\
\hline 551. the spleen decoction \\
\hline 552. (to) treat disorders \\
\hline 553. clinical efficacy \\
\hline 554. essential oils \\
\hline 555. TCM theory \\
\hline 556. (the) upper back \\
\hline 557. aconiti carmichaeli praeparata \\
\hline 558. codonopsis pilosulae \\
\hline 559. dysenteric disorder \\
\hline 560. fluid metabolism \\
\hline 561. fortify the spleen \\
\hline 562. herbal products \\
\hline 563. in the epigastrium \\
\hline 564. number of patients \\
\hline 565. semen plantaginis \\
\hline 566. sense organs \\
\hline 567. swollen tongue \\
\hline 568. tonifies the kidneys \\
\hline 569. turmoil disorder \\
\hline 570. (a) fing of distension \\
\hline
\end{tabular}


Continued

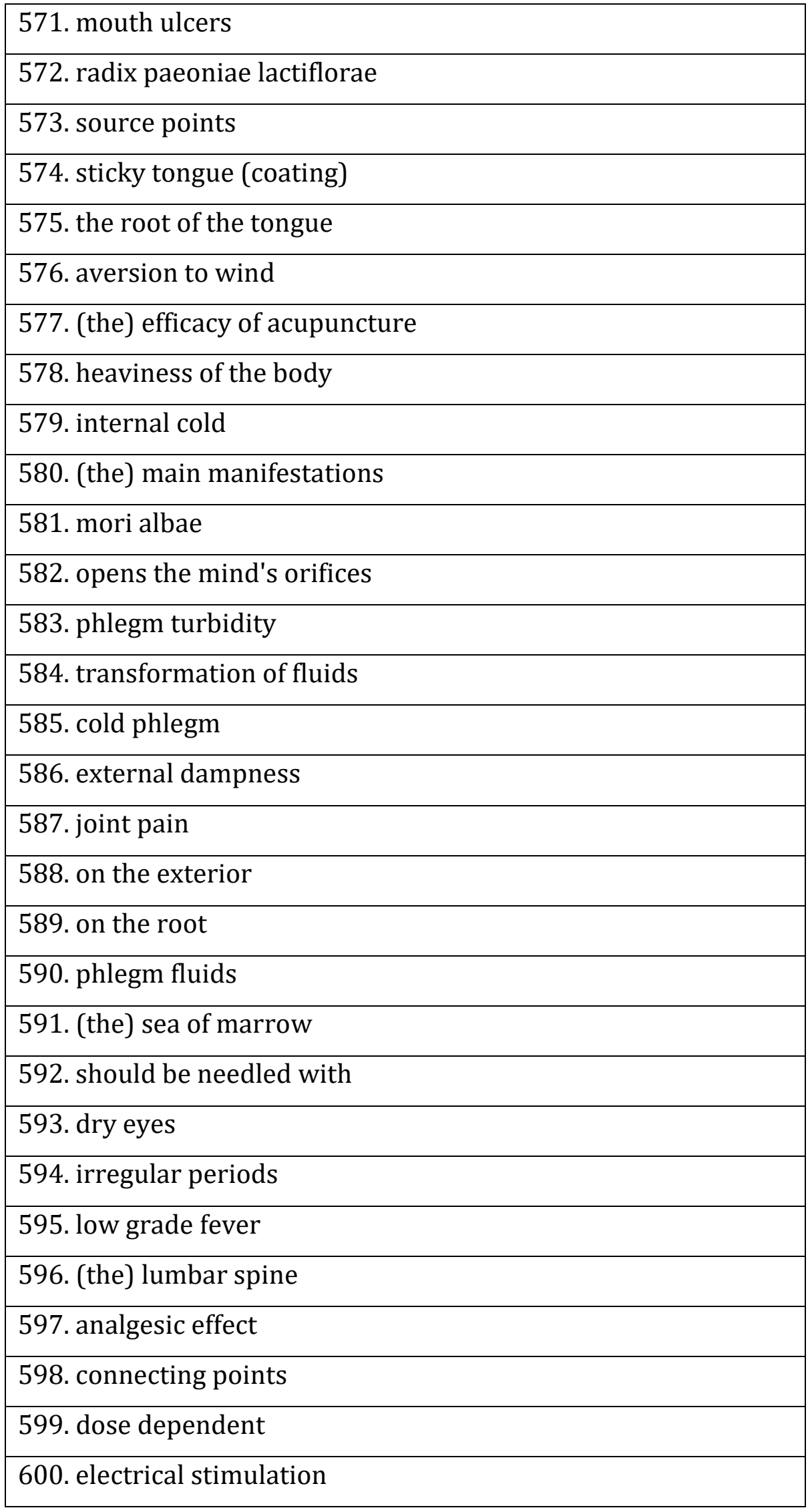


Continued

\begin{tabular}{|l|}
\hline 601. excess type \\
\hline 602. fine powder \\
\hline 603. forsythiae suspensae \\
\hline 604. fructus ziziphi jujubae \\
\hline 605. rhizoma acori graminei \\
\hline 606. tonifies spleen \\
\hline 607. animal models \\
\hline 608. cerebral ischemia \\
\hline 609. floating pulse \\
\hline 610. stabbing pain \\
\hline 611. wind dampness \\
\hline 612. deep needling \\
\hline 613. mania depression \\
\hline 614. opens the chest \\
\hline 615. radix paeoniae rubra \\
\hline 616. resolving phlegm \\
\hline 617. TCM syndromes \\
\hline 618. tuber curcumae \\
\hline 619. viral infection \\
\hline 620. dry skin \\
\hline 621. internal medicine \\
\hline 622. physical therapy \\
\hline 623. radix codonopsis pilosulae \\
\hline 624. shoulder pain \\
\hline 625. cardiovascular disease \\
\hline 626. endothelial cells \\
\hline 627. internal organ \\
\hline 628. ischemic stroke \\
\hline 629. lung cancer \\
\hline
\end{tabular}


Continued

\begin{tabular}{|l|}
\hline 631. pattern identification \\
\hline 632. semen persicae \\
\hline 633. the liver decoction \\
\hline 634. accumulation point \\
\hline 635. calms the spirit \\
\hline 636. cold herbs \\
\hline 637. deficiency type \\
\hline 638. fine pulse \\
\hline 639. fructus crataegi \\
\hline 640. fructus forsythiae \\
\hline 641. knee pain \\
\hline 642. liver heat \\
\hline 643. lung deficiency \\
\hline 644. myocardial infarction \\
\hline 645. (the) needling sensation \\
\hline 646. radix polygoni multiflori \\
\hline 647. recurrent miscarriage \\
\hline 648. remove obstructions (from) \\
\hline 649. sudden onset \\
\hline 650. the luteal phase \\
\hline
\end{tabular}




\section{Appendix 14. Word association task}

\section{Word association task}

This task contains target 20 words. Each target word (in bold) has eight (8) options. Please choose four words of the eight options which you think are most closely associated to the following words in the context of Traditional Chinese medicine (TCM). Put a tick $(\sqrt{ })$ in the box for each option you choose. Here is an example:
joint
$\square$ A. qi
$\square$ B. tendon
$\square$ C. skeleton
D. blood
$\square$ E. pain
$\square$ F. heart
$\square$ G. shoulder
$\square$ H. hepatitis

You might answer the task like this:
joint
$\checkmark$ A. qi
$\checkmark$ B. tendon
$\square$ C. skeleton
$\checkmark$ D. blood
$\square$ E. pain
$\square$ F. heart
$\square$ G. shoulder
$\square$ H. hepatitis

Think aloud: As you make your choices, I would like you to talk about your choices and why you chose (or did not choose) each one. 


\section{1. mind}

$\square$ A. essence

B. qi

C. consciousness

D. heart

E. insomnia

$\square$ F. calm

G. hiccup

$\square$ H. feelings

2. root
A. herb
B. chronic
C. acute
D. manifestation
E. treat
$\square$ F. abdomen
$\square$ G. cause
$\square$ H. strengthen

\section{3. spleen}
A. lung
B. digestion
C. stomach
D. blood
E. saliva
$\square$ F. tongue
G. deficiency
$\square$ H. eyes

\section{4. channel}
$\square$ A. organ
B. penicillin
C. acupoint
D. collaterals

$\square$ E. connecting

$\square$ F. liver

$\square$ G. oedema

$\square$ H. stomach

\section{5. skin}
$\square$ A. wheezing
B. tinnitus
C. tissue
$\square$ D. outer layer

$\square$ E. dry

$\square$ F. disease

$\square$ G. surface

$\square$ H. muscle

\section{6. qi}
A. spleen
B. yang
C. vision
$\square$ D. blood
$\square$ E. defensive
$\square$ F. movement
$\square$ G. zong
$\square$ H. stagnant

\section{7. throat}
$\square$ A. mouth
B. neck
C. nasopharynx
$\square$ D. kidney channel
$\square$ E. dry
$\square$ F. menopause
$\square$ G. sore
H. backache 


\section{8. acupuncture}

$\square$ A. needle

B. alternative therapy

C. acupressure

D. moxibustion

E. treatment

F. auricular

G. points

$\square$ H. stimulation

\section{9. blood}
$\square$ A. fluid
B. yin
C. heart
D. leukaemia
E. gallbladder
$\neg$ F. red
G. cool
H. liver

\section{0. knee}
A. leg
B. joint
C. respiration
D. bend
E. injury
F. ear
G. soreness
H. pain

\section{1. disharmony}
A. argument
B. disease
C. imbalance
D. wind

$\square$ E. vessel

$\square$ F. internal

$\square$ G. pattern

$\square \mathrm{H}$. liver

\section{2. cancer}
$\square$ A. tumour
B. toothache
C. malignant
$\square$ D. stroke

$\square$ E. lung

$\square$ F. colorectal

$\square$ G. gastric

$\square$ H. chemotherapy

\section{3. protein}
$\checkmark$ A. nutrient
B. egg
C. glucose
D. enzyme

$\square$ E. urine

$\square$ F. expression

$\square$ G. antibody

$\square$ H. therapy

\section{4. urine}
$\square$ A. waste
B. stool
$\square$ C. kidney
$\square$ D. bladder
$\square$ E. yellow
$\square$ F. spasm
$\square$ G. cloudy
H. turbid 
15. cold

$\square$ A. fever

B. heat

C. yin

D. pathogenic factor

$\square$ E. wind

$\square$ F. herbs

$\square$ G. intestine

$\square$ H. limbs

16. diarrhoea

$\square$ A. spleen

B. heart

C. borborygmus

D. stool

E. dampness

F. ceaseless

$\square$ G. dizziness

$\square$ H. chronic

17. phlegm

$\square$ A. throat

B. mucus

C. damp

D. cough

E. wind

$\square$ F. turbid

$\square$ G. heat

$\square$ H. hepatitis

\section{8. lumbar}
A. cervical
B. vertebrae

$\square$ C. diabetes

$\square$ D. back

$\square$ E. soreness

$\square$ F. pain

$\square$ G. spine

$\square$ H. region

\section{9. tonify}
A. deficiency
B. harmony
C. nourish
$\square$ D. expel
$\square$ E. bladder
$\square$ F. spleen
$\square$ G. qi
$\square$ H. kidney

\section{0. tang}

$\square$ A. pungent odour

$\square$ B. bitter

C. decoction

$\square$ D. herbs

$\square$ E. organ

$\square$ F. water

$\square$ G. chest

$\square$ H. cheng qi 


\section{Appendix 15. Ethics approval}

Te Whare Wánanga o te úpoko O Te iKa a máui

MEMORANDUM
Phone $\quad 0-4-4635480$

Email susan.corbett@vuw.ac.nz

\begin{tabular}{l|l}
\hline TO & Cailing Lu \\
\hline COPY TO & Averil Coxhead \\
\hline FROM & AProf Susan Corbett, Convener, Human Ethics Committee \\
\hline DATE & 27 April 2017 \\
\hline PAGES & 1 \\
\hline SUBJECT & $\begin{array}{l}\text { Ethics Approval: 24545 } \\
\text { Investigating learning and word associations of technical } \\
\text { vocabulary in Traditional Chinese Medicine }\end{array}$ \\
\hline
\end{tabular}

Thank you for your application for ethical approval, which has now been considered by the Standing Committee of the Human Ethics Committee.

Your application has been approved from the above date and this approval continues until 1 July 2018. If your data collection is not completed by this date you should apply to the Human Ethics Committee for an extension to this approval.

Best wishes with the research.

Kind regards

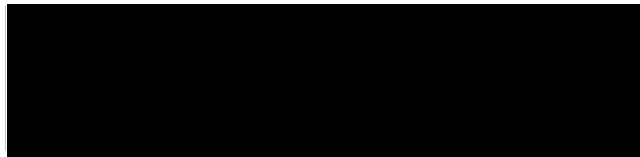

Susan Corbett

Convener, Victoria University Human Ethics Committee 


\section{Appendix 16. Information sheet}

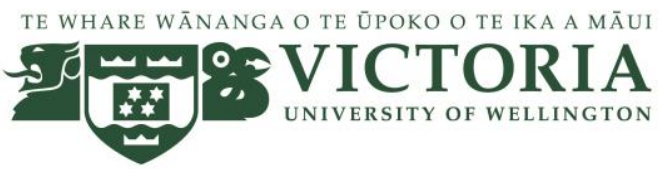

Project title: Investigating learning and word associations of technical vocabulary in Traditional Chinese Medicine INFORMATION SHEET FOR PARTICIPANTS

Thank you for your interest in this project. Please read this information before deciding whether or not to take part. If you decide to participate, thank you. If you decide not to take part, thank you for considering my request.

\section{Who am I?}

My name is Cailing Lu and I am a Doctoral student in Applied Linguistics at Victoria University of Wellington. This research project is work towards my thesis.

\section{What is the aim of the project?}

This project aims to find out whether you have difficulty in understanding technical vocabulary of Traditional Chinese Medicine and how you understand the given technical words. This research has been approved by the Victoria University of Wellington Human Ethics Committee 0000024545.

\section{How can you help?}

If you agree to take part, I will interview you in a meeting room on your campus. I will ask you questions about technical vocabulary and ask you to complete a short written task. During the task, I will ask you to 'think aloud' as you work, so I can find out more about what you are thinking as you are doing the task. The whole process will take about one hour. I will record the interview and task and write it up later. You can stop at any time, without giving a reason. You can withdraw from the study by contacting me at any point before 01 August, 2017. If you withdraw, the information you provided will be destroyed or returned to you.

\section{What will happen to the information you give?}

This research is confidential. This means that the researchers named below will be aware of your identity but the research data will be aggregated and your identity will not be disclosed in any reports, presentations, or public documentation. Only my supervisors and I will read the notes or transcripts of 
the interview and at the task results. The interview transcripts, summaries and any recordings will be kept securely and destroyed four years after the research ends.

\section{What will the project produce?}

The information from my research will be used in my PhD thesis/report.

\section{If you accept this invitation, what are your rights as a research participant?}

You do not have to accept this invitation if you don't want to. If you do decide to participate, you have the right to:

- $\quad$ choose not to answer any question;

- $\quad$ ask for the recorder to be turned off at any time during the interview and task;

- $\quad$ withdraw from the study before 01 August, 2017;

- $\quad$ ask any questions about the study at any time;

- $\quad$ receive a copy of your interview recording

- $\quad$ read over and comment on a written summary of your interview;

- $\quad$ agree on another name for me to use rather than your real name;

- be able to read any reports of this research by emailing the researcher to request a copy.

If you have any questions or problems, who can you contact?

If you have any questions, either now or in the future, please feel free to contact either:

\section{Student:}

Name: Cailing Lu

University email address:

Cailing.Lu@vuw.ac.nz

\author{
Supervisor: \\ Name: Averil Coxhead \\ Role: Primary supervisor \\ School: Linguistics and Applied \\ Language Studies \\ Phone: +64-4-463 5625 \\ Averil.Coxhead@vuw.ac.nz
}

\section{Human Ethics Committee information}

If you have any concerns about the ethical conduct of the research you may contact the Victoria University HEC Convener: Associate Professor Susan Corbett. Email susan.corbett@vuw.ac.nz or telephone +64-4-4635480. 


\section{Appendix 17. Consent form}

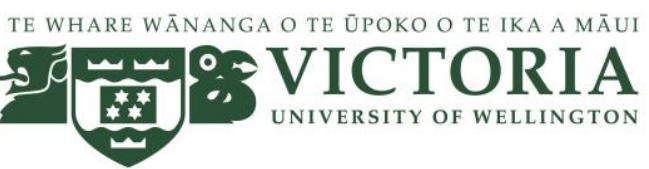

\section{Project title: Investigating learning and word associations of technical vocabulary in Traditional Chinese Medicine}

\section{CONSENT TO INTERVIEW}

This consent form will be held for 4 years.

Researcher: Cailing Lu, School of Linguistics and Applied Language Studies, Victoria University of Wellington.

- I have read the Information Sheet and the project has been explained to me. My questions have been answered to my satisfaction. I understand that I can ask further questions at any time.

- $\quad$ I agree to take part in an audio recorded interview and the written task.

I understand that:

- I may withdraw from this study at any point before 01 August, 2017, without giving any reason, and any information that I have provided will be returned to me or destroyed.

- $\quad$ The information I have provided will be destroyed four (4) years after the research is finished.

- Any information I provide will be kept confidential to the researcher and the supervisor. I understand that the results will be used for a PhD report and a summary of the results may be used in academic reports and/or presented at conferences.

- $\quad$ My name will not be used in reports, nor will any information that would identify me.

- I would like a copy of the transcript of my interview:

Yes $\square$ No

- I would like a summary of the findings:

Yes $\square$ No

Signature of participant: 
Name of participant:

Date:

Email address: 
Appendix 18. Distribution of the most frequent 100 technical words in the Frequency-Transparency Framework

\begin{tabular}{|c|c|c|c|}
\hline $\begin{array}{l}\text { High-frequency } \\
\text { and opaque }\end{array}$ & $\begin{array}{l}\text { High-frequency } \\
\text { and transparent }\end{array}$ & $\begin{array}{l}\text { Low-frequency } \\
\text { and transparent }\end{array}$ & $\begin{array}{l}\text { Low-frequency } \\
\text { and opaque }\end{array}$ \\
\hline $\begin{array}{l}\text { blood } \\
\text { heat } \\
\text { heart } \\
\text { cold } \\
\text { point } \\
\text { points } \\
\text { point } \\
\text { wind } \\
\text { stomach } \\
\text { channel } \\
\text { damp } \\
\text { formula } \\
\text { dampness } \\
\text { fire } \\
\text { pattern } \\
\text { control } \\
\text { cases } \\
\text { channels } \\
\text { chest } \\
\text { clear } \\
\text { vessel } \\
\text { water } \\
\text { mind } \\
\text { stasis } \\
\text { patterns } \\
\text { dry } \\
\text { treat } \\
\text { case } \\
\text { organ } \\
\text { factors }\end{array}$ & $\begin{array}{l}\text { treatment } \\
\text { used } \\
\text { patients } \\
\text { group } \\
\text { medicine } \\
\text { symptom } \\
\text { study } \\
\text { body } \\
\text { tongue } \\
\text { clinical } \\
\text { lower } \\
\text { use } \\
\text { effect } \\
\text { patient } \\
\text { disease } \\
\text { results } \\
\text { effects } \\
\text { groups } \\
\text { using } \\
\text { add } \\
\text { related } \\
\text { trials } \\
\text { red } \\
\text { data } \\
\text { method } \\
\text { significant } \\
\text { function } \\
\text { western } \\
\text { main } \\
\text { skin } \\
\text { condition } \\
\text { cause } \\
\text { level } \\
\text { cells } \\
\text { feeling } \\
\text { compared } \\
\text { participants }\end{array}$ & $\begin{array}{l}\text { acupuncture } \\
\text { decoction } \\
\text { herbs } \\
\text { syndrome } \\
\text { rhizoma } \\
\text { chronic } \\
\text { herbal } \\
\text { fructus } \\
\text { abdominal } \\
\text { pathogenic }\end{array}$ & $\begin{array}{l}\text { qi } \\
\text { yin } \\
\text { liver } \\
\text { yang } \\
\text { deficiency } \\
\text { radix } \\
\text { kidney } \\
\text { spleen } \\
\text { phlegm } \\
\text { pulse } \\
\text { lung } \\
\text { zi } \\
\text { tang } \\
\text { shen } \\
\text { zhi } \\
\text { kidneys } \\
\text { stagnation } \\
\text { fluid } \\
\text { fu } \\
\text { lungs } \\
\text { cao } \\
\text { bladder } \\
\text { pi }\end{array}$ \\
\hline
\end{tabular}




\section{Appendix 19. Interview guide}

\section{Asking for demographic information (questions to open the interview)}

Hello. My name is Cailing Lu. I'm going to start this interview with a couple of questions about you and then we will move on to a task about Chinese Medicine.

1). What's your name?

2). How many years have you been studying Traditional Chinese Medicine?

3). Where are you from, what is your mother tongue, how many languages do you speak?

2. Now we are going to do a paper-and-pencil task about TCM. (see the attached sheet on this word association task).

Thank you for doing the task. Now we are going to finish with a couple of questions about you. What do you plan to do after you finish your study? 


\section{Appendix 20. Sample of interview data from a Chinese TCM learner (English Translation)}

I: Interviewer

P1: Participant

I: Ok, now let's start the interview? Can I please ask your name?

P1: Um, $\mathrm{xx}$.

I: How many years have you been learning TCM?

P1: Ah, four years, now it's the fifth year.

I: Which city are you from?

P1: Um, Heilongjiang.

I: So what's your mother tongue?

P1: Chinese.

I: How many languages can you speak?

P1: Chinese and English, these two.

I: Ok, now let's do the task. It's a word association task, there is no standard right or wrong answers, it looks at your understanding of the target cue words.

P1: Ok.

I: So let's start from the first one.

P1: Is this one?

I: Yes. When you finish the first one, please tell me.

P1: Um, tell you about the choices?

I: Yup.

P1: (No.1 mind). The first one I chose A(essence) B(qi) D (heart) and H (feelings).

I: ABDH, ok, can I please ask why you chose ABD and H?

P1: I think heart governs mind, so I wanted to choose D (heart) and H (feelings). Then I feel that qi, blood, essence, fluid, they are associated with, that, that called ethereal soul, mind, intellect, corporeal soul and will power. So I chose B (qi) and A (essence).

I: Good, so why didn't you choose c (consciousness)?

P1: I just feel that consciousness sort of thing is related to western psychology, so I didn't choose it.

I: Can you tell me what is E (insomnia) in Chinese?

P1: E, that's insomnia, isn't that? 
I: Yup, and why didn't you choose G (hiccup)?

P1: I feel that mind is more about spiritual aspect, but feel that G is more physical sort of thing, so I didn't choose it.

I: cool, now let's look at the second one (root).

P1: Ok, the second one I chose A (herb), E (treat), G (cause) and H(strengthen).

I: Can I please ask how you would translate root?

P1: root, right, the root of plants.

I: Yup, just tell me what that was, so in TCM, the most associative four, can you please tell me why you chose A, E, G, H?

P1: There are many herbs, we use the root as medicine, so I think it is associated with herbs, then I chose A. Then it can normally be used to treat diseases, so I chose E. Then if you treat diseases, there must be a cause of disease, so I chose G. The last one is that I think of Ginseng, which can tonify qi or sort of that, so I chose $\mathrm{H}$.

I: So have you heard of D? It is manifestation.

P1: Is that manifestation?

I: Yup, so have you heard of root and manifestation in TCM?

P1: Ah, yup.

I: So if you understood it as root (in root and manifestation), how would you choose?

P1: If so, I would choose B, C, D, and G.

I: Why did you choose C?

P1: That is if it is acute diseases, treat the manifestation; if it is chronic diseases, treat the root. So I wanted to choose B and C, and they should have a cause of disease. So I chose G.

I: ok, let's look at the next one (No.3 spleen)?

P1: ok, for the third one, I chose B, C, D, and G.

I: Good, can you tell me why you chose B, C, D, and G?

P1: That is, spleen and stomach are internally and externally connected, so I chose stomach. Then, spleen is the ... so I chose D. And spleen and stomach are related to digestion, so I choose B. And we often say spleen yang deficiency, this is what I often heard, so I chose G.

I: So why did you think that lung is not? 
P1: Because when I saw lung at the beginning, I thought of respiration that sort of things, so I didn't choose it.

I: So in TCM theory, have you heard of the generating relationship?

P1: Yeah, I heard of that.

I: So you know the generating relationship, but you chose the associations which are close to the disease and patterns, is this what you mean.

P1: Yup.

P1: (No.4 channel) I only found three for this one.

I: Ok, that's ok.

P1: I chose C, D, E. That is, I feel, that I saw it when talking about channels and collaterals, they used this channel. And channels and collaterals so I chose D. Then, it connects organs, so I chose E. Then I thought channels and collaterals are related to acupuncture, so I chose C. And others, I didn't find associations, so I didn't choose them.

I: So why didn't you think channels are not associated with organs?

P1: At that moment, I thought channels and collaterals can't be divided into organs, so I didn't choose it.

I: Ok, so why did you think $\mathrm{F}$ and $\mathrm{H}$ are not associated?

P1: I just thought they are not inside the organs, so I didn't tick these two.

I: ok, so have you heard of something like spleen channel, and stomach channel?

P1: Ah, if talking about spleen channel and stomach channel, they are actually related.

I: Good, let's look at the next one (No.5 skin).

P1: No. 5 I chose C, F, G, H.

I: Cool, can you please tell me why you chose these?

P1: That is, I feel that skin is one kind of tissue, it is made up of different tissues, so I chose $\mathrm{C}$. Then, I thought that it is the surface, so I chose G. Then I feel it is kind of close to the muscle, so I chose H. Then I feel that normally cold invades the skin, so I chose F.

I: Good, so you chose G but why didn't you chose D?

P1: At the beginning I didn't know what that word mean.

I: Which word? 
P1: That, I feel, when I saw D, I need to think about it, but the meaning of G is more obvious, so I chose D.

I: good, let's look at the next one (No.6 qi)?

P1: I chose five for this one, that is B, D, E, F, and $\mathrm{H}$.

I: So which is the furthest one in these five? I mean if you need to exclude one, which one will you exclude?

P1: I excluded the fourth on (D. blood).

I: Yeah, so you excluded blood, can you please tell me why?

P1: I firstly chose B, D, and F, thinking that qi has certain movement, then qi and blood have some relationship, and qi corresponds to yang, so I choses these. Later, I thought that $\mathrm{E}$ and $\mathrm{H}$ are symptoms caused by deficiency of qi or qi stagnation, so I thought those two belong to the same category, if I chose one, I have to choose the other one. So I chose those.

I: So if you have to exclude on, you excluded blood, right?

P1: Yes.

I: Why don't you choose $\mathrm{C}$ then?

P1: C, I couldn't think of their association.

I: What will you translate $\mathrm{C}$ in Chinese?

P1: Em, how should I translate it, is it vision?

I: Yup, so why did you think it isn't associated with spleen?

P1: They are associated in some way, but I think it can associate with any zang and fu organs. Looking in this way, I don't think it is closely related to spleen.

I: Good, then No.7 (throat).

P1: Em, No.7 I chose A, B, D, and G. I chose A B because they are close in terms of location, that is I thought of location, then sore throat, so I thought of G. Then that channels and collaterals, so I chose D.

I: You said you chose A and B because they are close in terms of location, but why didn't you choose C?

P1: C, I don't know this word.

I: Ok, you said sore throat so you chose G, but why didn't you choose E?

P1: Em, because at that time I thought that I have sore throat often, sore is comparatively obvious, so I chose G. Dry is not that obvious.

I: So do you know what F means? 
P1: F means something like the period is not normal, does it? I'm not quite sure.

I: Ok, no worries.

P1: Em, No.8 (acupuncture), I chose A, B, D, E.

I: Good, why did you choose A, B, D, E?

P1: That is, I feel that when talking about acupuncture, moxibustion is followed, so I chose the fourth one first. Then normally needles are used in acupuncture, so I chose A. Then I feel that it is one kind of treatment, so I chose E; and now we usually categorize acupuncture as alternative therapy, so I chose the second one. I: Why didn't you choose C?

P1: I feel that it is something in the acupuncture, I mean, something in-depth, but I chose what I firstly thought about.

I: Then, why didn't you choose G (point)?

P1: For G, I think if there is an affix, acu, added to it, in that case, I would choose it. If it is by itself, I probably thought of others first, so I didn't choose it.

I: Good, let's look at the next one?

P1: No.9 (blood) I chose A, B, C, H.

I: Em, can you please tell me why you chose those?

P1: Firstly, that blood corresponds to yin, so I chose B. Then, it dose belong to body fluid, so I chose A. Then I thought of that heart governs blood and liver stores blood, so I chose $\mathrm{C}$ and $\mathrm{H}$.

I: Then why didn't you choose D?

P1: I feel that B is talked more in western medicine. Normally in TCM, we wouldn't talk about it in this way. Probably there is another term to refer to it in TCM, so I didn't choose it.

I: Then, why didn't you choose G?

P1: G, at that time I feel their association is a bit far. Actually I wanted to choose F, but comparatively speaking, I didn't choose it.

I: Does that mean, compared to the other options, you thought the others have closer association?

P1: Yes.

P1: (No.10 knee) Em, I chose A, B, D, E. That is, I feel, seeing knee, I thought about leg, then I thought about joints, so I chose A, B. Then knee can not only be painful, but also get injured, that sort of symptoms. $\mathrm{E}$ is more broader, so I chose $\mathrm{E}$. 
I: Then, why didn't you choose G?

P1: Because I feel it is narrowly focused, and E is broader.

I: Good.

P1: (No.11 disharmony) Em, this item I chose B, C, F, G.

I: Good, can you please tell me why?

P1: That is, that yin-yang disharmony of human body can lead to disease, so I chose B. Then, this and C, they are synonyms, that is they have similar meanings, so I chose C. Then I feel that it can probably be internal of body itself, or it can be external environment, so I chose F. Then that one refers to the internal one, then I thought this kind of yin-yang disharmony can also be one disease pattern, so I chose G.

I: Then, why didn't you choose D?

P1: I also thought that D can be one of the causes of disharmony, but I feel there might be many other causes, so I didn't choose it.

I: Good.

P1: (No.12 cancer) I chose A, C and H for this one. Normally, cancer and tumor are talked about together, so I chose A. Then, it can be benign or malignant, so I chose C. Then, it can be treated with chemotherapy, so I chose $\mathrm{H}$.

I: Why didn't you choose G?

P1: G, I don't know this word.

I: Ok, why didn't you choose E?

P1: Because I feel that E and F belong to one type of cancer, they are too narrow, so I didn't choose them.

P1: (No.13 protein) Em, I chose A, D, F, G for this item. I feel that protein is nutrient, so I chose A. Then it is related to DNA expression, so I chose F. Then enzyme and antibody, I feel they are related to protein, so I chose D and G.

I: Why didn't you choose B?

P1: Because I feel that it is only one type of stuff which is rich in protein, so I didn't choose it.

I: Why didn't you choose E?

P1: I wanted to choose it at that moment because of urine protein, but then I feel comparatively speaking the others are more closely associated.

I: So if you were given the fifth choice, would you choose E? 
P1: I would.

I: Good.

P1: (No.14 urine) Em, I chose A, C, D, E for this one. That is, when I think about it, I think about it is related to kidney and bladder, then I chose $\mathrm{C}$ and $\mathrm{D}$. Then $\mathrm{E}$, the color in normal condition. Then I think about it was kind of waster expelled from the body, so I chose A. Then I feel that $\mathrm{H}$ is also ok, but A is more comprehensible, and more commonly used, so I chose A.

I: why didn't you choose B?

P1: That, I think, both of them are waste, but they are from different ways, so I didn't choose it.

I: Good.

P1: (No.15 cold) I chose B, D, E, F for this one. I feel that wind, cold, summer-heat, dampness, and dryness, they are all pathogenic factors, so I chose the fourth one. Then I feel it usually comes together with wind. That is get invaded by cold and wind externally, so I chose E. Then, normally we will prescribe herb decoction for treatment, so I chose F. Because it is cold, so it might use hot herbs. Oh, no, I made a mistake, I took heat as hot.

I: You can change one.

P1: Then I choose a, because it can lead to fever and that sort of things.

I: Why didn't you choose H?

P1: Because the soreness of limbs is not as common as fever. I feel fever is especially, especially for kids, so I chose A.

I: So when you saw cold, what did it mean to you?

P1: Actually, I thought of cold (as opposite to hot), then I thought of cold (as pathogenic factors) in TCM.

I: Good.

P1: (No. 16 diarrhoea) Um, I chose five for this one, they are A, D, E, G, H. First, I thought that diarrhoea should be associated with $\mathrm{D}$ (stool). No, it should belong to one type of that. Then, normally it is related to spleen, and spleen dampness, so I chose A and E. Then, dampness, I thought that it can lead to sleepiness and tiredness, and it is long-term, so I chose G and H.

I: So why didn't you choose F?

P1: F, this word looks unfamiliar to me. 


\section{I: What about C?}

P1: The same to C.

I: What if you know $\mathrm{C}$ means borborygums, would you choose it?

P1: Yes, I would.

I: So if you know the meaning of $\mathrm{C}$, and ask you to exclude one of those, which one would you exclude?

P1: If I know it, I would exclude both $\mathrm{G}$ and $\mathrm{H}$, because I think $\mathrm{C}$ is more closely associated with it.

I: $0 \mathrm{k}$.

P1: (No.17 phlegm) I chose A, C, and D for this one.

I: Why did you choose only three?

P1: Because I didn't know B, and I think, for E and G, I just think phlegm is mostly related to dampness, so I think it is not closely associated with E and G, so I didn't choose them. Then I think hepatitis is rarely mentioned in TCM, so I didn't choose it.

I: So have you heard of wind phlegm?

P1: I actually seldom meet it.

I: Good, next one (No.18 lumbar).

P1: I don't really know this word.

I: No worries, let's look at the next one (No.19 tonify)

P1: I chose five for this one, they are A, C, F, G, H.

I: Yup, why did you choose five?

P1: I just think that there must be deficiency if tonify and strengthen that sort of thing is used. Then, I thought tonify is used for yang, then C nourish is used for yin, so I chose $\mathrm{C}$ as well. Then I thought this kind of yang is often related to qi, so I chose G. Then, tonify kidney, tonify spleen is often used, so I thought if I chose F, I need to choose $\mathrm{H}$ as well.

I: So if you are asked to rank them, for example, which one is closest, the second closest, how would you rank them?

P1: Um, the closest, I might choose A, then it's G, then F and H, which are the same. The last one would be C. So if I need to exclude one, I would exclude C.

I: Good, the last one (No. 20 tang).

P1: Is this one an English word? It seems that I don't know this. 
I: So you thought it was an English word after first glance?

P1: Yup, then I feel I don't know this word.

I: That is, when you read literature in TCM, you would often see...

P1: Pinyin?

I: Yes.

P1: That is a Pinyin, Ah, I know it, so I chose A, C, D, F.

I: Um, can you please tell me why?

P1: That is tang, so I think C (decoction) also means tang. Then it often used herbs, and used water to boil it. Then the taste and smell are very special, unique to herb medicine.

I: Why didn't you choose E and G then?

P1: This is decoction; I just feel they are not closely related to decoction. Actually $\mathrm{B}$ is ok, because it is often bitter,

I: So you would choose B as the fifth one?

P1: Yes.

I: Good, thank you very much for completing this task, can I ask a couple more questions?

P1: Yes.

I: I want to know why did you choose this major?

P1: Because at that time I had a look at the syllabus, and found it interesting because it has a lot of chemical and biological courses.

I: Cool, thank you very much! 
